\title{
Identification of significant pathways in gastric cancer based on protein-protein interaction networks and cluster analysis
}

\author{
Kongwang $\mathrm{Hu}^{1}$ and Feihu $\mathrm{Chen}^{2}$ \\ ${ }^{1}$ Department of General Surgery, The First Affiliated Hospital of Anhui Medical University, \\ Anhui, P.R. China. \\ ${ }^{2}$ School of Pharmacology, Anhui Medical University, Anhui, P.R. China.
}

\begin{abstract}
Gastric cancer is one of the most common and lethal cancers worldwide. However, despite its clinical importance, the regulatory mechanisms involved in the aggressiveness of this cancer are still poorly understood. A better understanding of the biology, genetics and molecular mechanisms of gastric cancer would be useful in developing novel targeted approaches for treating this disease. In this study we used protein-protein interaction networks and cluster analysis to comprehensively investigate the cellular pathways involved in gastric cancer. A primary immunodeficiency pathway, focal adhesion, ECM-receptor interactions and the metabolism of xenobiotics by cytochrome P450 were identified as four important pathways associated with the progression of gastric cancer. The genes in these pathways, e.g., ZAP70, IGLL1, CD79A, COL6A3, COL3A1, COL1A1, CYP2C18 and CYP2C9, may be considered as potential therapeutic targets for gastric cancer.
\end{abstract}

Key words: graph clustering, pathway crosstalk, protein-protein interaction network.

Received: March 12, 2012; Accepted: May 4, 2012.

\section{Introduction}

Gastric cancer is one of the most common malignancies worldwide (Lin et al., 2007b). Surgical resection is the only effective treatment for this cancer, although current surgical therapeutic strategies are far from optimal and most patients are diagnosed with late-stage disease when surgical intervention is of limited use (D’Ugo et al., 2009). Chemotherapy has been applied as a neoadjuvant treatment to improve the curative resection rate or to achieve longterm survival in patients with unresectable gastric cancer. The prognosis, however, is still unsatisfactory, with an overall five-year survival rate of $24 \%$ (Kanai et al., 2003). Hence, there is an urgent need for new therapeutic strategies.

Recently, several molecular alterations involving various pathways have been implicated in the development and late-stage progression/metastasis of gastric cancer. For example, there is emerging evidence that the Wnt signaling pathway may contribute to gastric carcinogenesis by stimulating the migration and invasion of gastric cancer cells (Kurayoshi et al., 2006). Persons with germ-line mutations in the APC tumor suppressor gene have a 10 -fold increased risk of developing gastric cancer when compared with nor-

Send correspondence to Feihu Chen. School of Pharmacology, Anhui Medical University, Meishan Road 81, 230032 Hefei, Anhui, P.R. China. E-mail: feihuchensci@gmail.com. mal persons (Offerhaus et al., 1992). $\beta$-catenin is frequently mutated in gastric cancer (Clements et al., 2002). In addition, frizzled receptor E3 (FzE3) is over-expressed in $75 \%$ of gastric carcinoma tissues and secreted frizzled related protein (hsFRP) is down-regulated in 16\%, suggesting that alterations in FzE3 and hsFRP expression are frequent in this pathology (To et al., 2001). Activation of the hedgehog pathway is another important mechanism associated with aggressive gastric cancer. The sonic hedgehog (Shh) transcript is restricted to cancer tissue whereas Gli1 and human patched gene 1 (PTCH1) are expressed in cancer cells and the surrounding stroma. The treatment of gastric cancer cells with 3-keto-N-aminoethylaminocaproyldihydrocinnamoyl-cyclopamine, a hedgehog signaling inhibitor, decreases the expression of Gli1 and PTCH1 and results in cell growth inhibition and apoptosis (Ma et al., 2005). The high recombinant Shh-induced migration and invasiveness of gastric cancer cells is mediated by tissue growth factor-beta (TGF- $\beta$ ) acting through the ALK5-Smad3 pathway (Yoo et al., 2008). The expression of lysyl oxidase-like 2 (LOXL2), which can promote tumor cell invasion via the Src kinase/focal adhesion kinase (Src/FAK) pathway, is markedly increased in gastric cancer (Peng et al., 2009). The loss of embryonic liver fodrin (ELF) can disrupt TGF- $\beta$-mediated signaling by interfering with the localization of Smad3 and Smad4 and leads to the development of gastric cancer (Kim et al., 2006). 
An increased concentration of BMP-2 strongly enhances the motility and invasiveness in gastric cancer cells. The stimulation of BMP-2 in gastric cancer cells induces a full epithelial-mesenchymal transition (EMT) characterized by Snail induction, E-cadherin reorganization and the down-regulation and up-regulation of mesenchymal and invasiveness markers through the activation of phosphatidylinositol 3 (PI-3) kinase/Akt (Kang et al., 2010). Cysteine-rich 61 (Cyr61) may contribute to the progression of gastric cancer by promoting tumor cell motility/invasion through the up-regulation of cyclooxygenase-2 (COX-2) in an integrin avh3/NF-kB-dependent manner. Interleukin-6 induces gastric cancer cell line AGS cell invasion through activation of the c-Src/RhoA/ROCK signaling pathway (Lin et al., 2007a).

The use of high-throughput approaches to dissect the molecular mechanisms and pathways that regulate the progress of gastric cancer is still comparatively rare. In this study, we used microarray data, protein-protein interaction (PPI) networks and cluster graph analysis to identify significant pathways involved in the development of gastric cancer. The characterization of genes and pathways involved in gastric cancer should be useful in identifying potential targets for the development of novel strategies for treating gastric carcinoma.

\section{Data and Methods}

\section{Data sources}

The KEGG (Kyoto Encyclopedia of Genes and Genomes) (Kanehisa, 2002) datasets were downloaded on February 19, 2011, at which time they contained 211 pathways and 5,385 genes. The PPI data were collected from the HPRD (Human Protein Reference Database) (Keshava Prasad et al., 2009), MINT (Molecular INTeraction Database) (Chatr-aryamontri et al., 2007) and BIOGRID (Biological General Repository for Interaction Datasets) (Stark et al., 2011). A total of 21,978 unique PPI pairs were obtained, of which 21,353 were from HPRD, 8,830 were from MINT and 19,243 were from BIOGRID. An ensemble PPI network was constructed by integrating three of the above PPI databases for humans, with at least two PPI databases being used to form an intersection (the PPI data are provided as Table S1 in Supplementary Material).

The gene expression profile data were accessed at the National Center for Biotechnology Information (NCBI) Gene Expression Omnibus (GEO) data repository using the accession number GSE2685. Samples of gastric cancer tissue and corresponding adjacent noncancerous tissue were obtained with the informed consent of patients who underwent gastrectomy at Jichi Medical College Hospital (Tochigi, Japan) (Hippo et al., 2002). Twentytwo gastric cancer tissue samples and eight noncancerous gastric tissue samples were analyzed with oligonucleotide microarrays (GeneChip Hu-GeneFL array; Affymetrix, Santa Clara, CA).

\section{Analysis of significant pathways based on cluster graph analysis}

The Limma eBayes analysis (Smyth, 2004) was used to assess the differential expression status of each gene. Background intensities were adjusted and the original expression datasets from all conditions were processed into expression estimates using the robust multiarray average (RMA) method (D'Souza et al., 2008) with the default settings implemented in R (version 2.12.1) (Gentleman et al., 2004); this was followed by construction of the linear model. The empirical Bayes approach was used to further justify these estimators; this process is equivalent to shrinking the estimated sample variances towards a pooled estimate and yields a far more stable inference when the number of arrays is small (Smyth, 2004). At least a two-fold change in expression and a $p$ value of $<0.05$ were considered as the threshold for defining differentially expressed genes (DEGs). Spearman's rank correlation (r) was used to assess the association between different DEGs. The level of significance was set at $r>0.75$ and the false discovery rate (FDR) at $<0.05$ (Strimmer, 2008). All statistical tests were done using the R program.

To identify co-expressed groups we used DPClus (Altaf-Ul-Amin et al., 2006). DPClus is a cluster graph algorithm that can extract densely connected nodes as a cluster and is based on density tracking and peripheral tracking of clusters. In this study, we used the overlapping-mode of the DPClus settings. For this analysis, we used the parameters "cluster property" (cp), a density value of 0.5 and a minimum cluster size of 5 (Fukushima et al., 2011). DAVID software (Huang da et al., 2008) was used for pathway enrichment analysis with $p<0.05$ selected as the threshold for gene clusters based on their hypergeometric distribution.

\section{Analysis of significant pathways and pathway crosstalk based on PPI networks}

Pathway crosstalk was defined as those pathways that had overlapping genes and edges. "Overlapping genes" meant that both of the pathways included these genes whereas "overlapping edges" meant that both pathways included the PPI interaction edges. Liu et al. (2010) have provided a detailed analysis of crosstalk relationships. The significance of a co-expressed gene pair in gastric cancer was assessed using Pearson's correlation coefficient and the corresponding $\mathrm{p}$ values, with the latter being mapped to the nodes and edges in the PPI network. The final identification of significant pathways was based on the extent of overlap of the pathways identified by the two methods (cluster graph analysis and PPI networks). 


\section{Results}

Identification of significant pathways based on screening for differentially expressed genes and cluster graph analysis

A publicly available microarray dataset (GSE2685) was downloaded from GEO and screened for DEGs. In the microarray analysis, 723 genes with a fold change $>2$ and $\mathrm{p}<0.05$ were identified as DEGs using the limma eBayes method. Based on the cutoffs established for $r(>0.75)$ and FDR $(<0.05)$ a correlation network was constructed that included 1032 relationships among 364 DEGs. At $r>0.75$, DPClus identified 22 clusters that ranged in size from 5 to 24 genes, with each cluster being connected to neighboring clusters (Figure 1). The significance of the clusters was as-

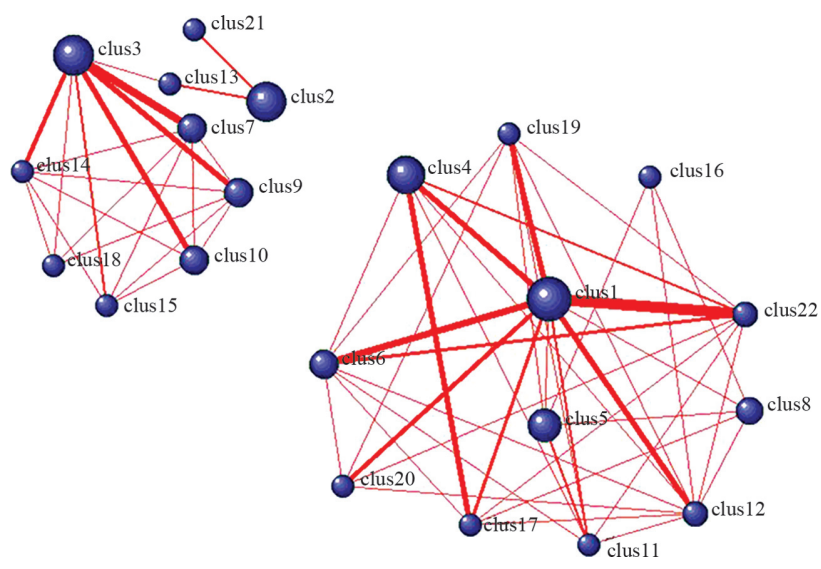

Figure 1 - Clustering of correlated modules in gastric cancer (threshold $r \geq 0.75$ ). The circles indicate clusters and the red lines (edges) indicate crosstalk (shared genes) between clusters. sessed by examining the over-represented pathways in these clusters (also known as pathway enrichment analysis). Table 1 shows the results of this analysis based the cluster graphs in Figure 1. Only clusters 1, 2, 3, 4, 8 and 19 contained enriched pathways.

Primary immunodeficiency (hsa05340) enriched in cluster 1 was connected with the metabolism of xenobiotics by cytochrome P450 (hsa00980), linoleic acid metabolism (hsa00591) and retinol metabolism (hsa00830), which were enriched in cluster 4 (Figure 1). Primary immunodeficiency (hsa05340) was also connected with the B cell receptor signaling pathway (hsa04662) that was enriched in cluster 19. Cluster 2, in which ECM-receptor interaction (hsa04512) and focal adhesion (hsa04510) were enriched, was indirectly connected with cluster 3 that included cell cycle (hsa04110), oocyte meiosis (hsa04114) and DNA replication (hsa03030).

\section{Identification of significant pathways and pathway crosstalk based on PPI networks}

Twenty significant pathways with $\mathrm{p}<0.05$ were detected using the KEGG pathways and PPI datasets (Table 2). Further analysis of these pathways revealed only 11 cases of crosstalk that involved nine significant pathways (Figure 2). Primary immunodeficiency (hsa05340) showed crosstalk with the ribosome (hsa03010) and chemokine signaling pathway (hsa04062). More importantly, cluster graph analysis and PPI networks identified primary immunodeficiency (hsa05340), focal adhesion (hsa04510), metabolism of xenobiotics by cytochrome P450 (hsa00980) and ECM-receptor interaction (hsa04512) as overlapping significant pathways with

Table 1 - Clusters showing pathway enrichment.

\begin{tabular}{|c|c|c|c|c|c|}
\hline Category & Term & Description & Count & p-value & FDR \\
\hline Cluster 1 & hsa05340 & Primary immunodeficiency & 3 & 0.0029 & 0.0675 \\
\hline Cluster 2 & hsa04512 & ECM-receptor interaction & 4 & 0.0003 & 0.0034 \\
\hline Cluster 2 & hsa04510 & Focal adhesion & 4 & 0.0043 & 0.0212 \\
\hline Cluster 3 & hsa04110 & Cell cycle & 5 & 0.0002 & 0.0003 \\
\hline Cluster 3 & hsa04114 & Oocyte meiosis & 3 & 0.0119 & 0.0805 \\
\hline Cluster 3 & hsa03030 & DNA replication & 2 & 0.0553 & 0.2331 \\
\hline Cluster 4 & hsa00591 & Linoleic acid metabolism & 2 & 0.0432 & 0.6968 \\
\hline Cluster 4 & hsa00830 & Retinol metabolism & 2 & 0.0819 & 0.6846 \\
\hline Cluster 4 & hsa00590 & Arachidonic acid metabolism & 2 & 0.0848 & 0.5497 \\
\hline Cluster 4 & hsa00980 & Metabolism of xenobiotics by cytochrome P450 & 2 & 0.0906 & 0.4734 \\
\hline Cluster 4 & hsa00982 & Drug metabolism & 2 & 0.0935 & 0.4116 \\
\hline Cluster 8 & hsa00190 & Oxidative phosphorylation & 2 & 0.0984 & 0.6801 \\
\hline Cluster 19 & hsa04662 & B cell receptor signaling pathway & 2 & 0.0293 & 0.1878 \\
\hline
\end{tabular}

Term represents the pathway identification (ID), Description is the pathway symbol and Count is the number of enriched pathways. The p value is the probability of obtaining a test statistic. The smaller the $\mathrm{p}$ value, the greater the number of enriched pathways. The False discovery rate (FDR) is a statistical method used to correct for multiple comparisons in multiple hypotheses testing; the smaller the FDR, the greater the correctness. ECM - extracellular matrix. 
Table 2 - Pathways showing significant crosstalk.

\begin{tabular}{|c|c|c|c|c|c|}
\hline Pathway ID & Description & Size & Node & Edge & p-value \\
\hline hsa00071 & Fatty acid metabolism & 42 & 2 & 2 & 0.0038 \\
\hline hsa00280 & Valine, leucine and isoleucine degradation & 44 & 2 & 2 & 0.0064 \\
\hline hsa00520 & Amino sugar and nucleotide sugar metabolism & 45 & 2 & 2 & 0.0107 \\
\hline hsa00534 & Glycosaminoglycan biosynthesis - heparan sulfate & 26 & 2 & 3 & 0.0301 \\
\hline hsa00910 & Nitrogen metabolism & 23 & 2 & 4 & 0.0113 \\
\hline hsa00980 & Metabolism of xenobiotics by cytochrome P450 & 70 & 4 & 8 & 0.0133 \\
\hline hsa03010 & Ribosome & 88 & 19 & 39 & 0.0438 \\
\hline hsa03060 & Protein export & 24 & 2 & 2 & 0.0113 \\
\hline hsa03420 & Nucleotide excision repair & 44 & 25 & 58 & 0 \\
\hline hsa04012 & ErbB signaling pathway & 87 & 3 & 3 & 0.0120 \\
\hline hsa04062 & Chemokine signaling pathway & 189 & 61 & 176 & 0 \\
\hline hsa04310 & Wnt signaling pathway & 151 & 5 & 6 & 0.0453 \\
\hline hsa04510 & Focal adhesion & 201 & 3 & 2 & 0.0038 \\
\hline hsa04740 & Olfactory transduction & 389 & 2 & 2 & 0.0384 \\
\hline hsa04930 & Type II diabetes mellitus & 47 & 5 & 6 & 0.0181 \\
\hline hsa04964 & Proximal tubule bicarbonate reclamation & 23 & 3 & 5 & 0.0145 \\
\hline hsa04512 & ECM-receptor interaction & 132 & 7 & 23 & 0.0307 \\
\hline hsa05144 & Malaria & 51 & 5 & 27 & 0.0480 \\
\hline hsa05322 & Systemic lupus erythematosus & 142 & 9 & 16 & 0.0180 \\
\hline hsa05340 & Primary immunodeficiency & 35 & 3 & 3 & 0.0332 \\
\hline
\end{tabular}

Description refers to the pathway name. Size is the number of genes contained in the KEGG gene sets. Edge and Node represent the number of edges and nodes of the pathways in the protein-protein interaction network that contain gene expression information. The p value indicates the dysregulation score for each pathway. ECM - extracellular matrix.

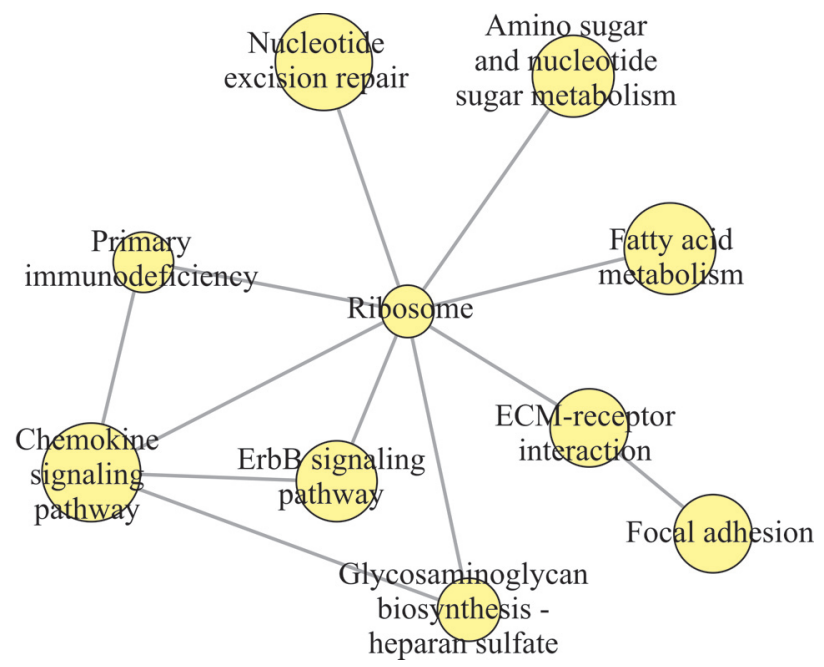

Figure 2 - Analysis of pathway crosstalk based on protein-protein interaction networks. The yellow circles indicate the pathways and the gray lines (edges) indicate the links between any two pathways.

considerable crosstalk, e.g., between focal adhesion (hsa04510) and ECM-receptor interaction (hsa04512) based on the PPI network. Cluster graph analysis showed that both of these two pathways were enriched in cluster 2 .

\section{Discussion}

Graph clustering or PPI-based pathway analysis (Hwang et al., 2008) has been successfully used to identify the underlying mechanisms associated with diseases. In this study, we used the same strategy to identify DEGs associated with gastric cancer and predict their underlying molecular mechanisms. Our cluster analysis showed that primary immunodeficiency (hsa05340) enriched in cluster 1 interacted not only with the metabolism of xenobiotics by cytochrome P450 (hsa00980), linoleic acid metabolism (hsa00591) and retinol metabolism (hsa00830) that were enriched in cluster 4, but also with the B cell receptor signaling pathway (hsa04662) enriched in cluster 19. These results indicated that the primary immunodeficiency pathway has several important roles in gastric cancer. Although additional pathways were observed in PPI-based pathway analysis, the primary immunodeficiency pathway still retained important roles in gastric cancer through crosstalk with the ribosome (hsa03010) and chemokine signaling pathway (hsa04062). In addition, focal adhesion (hsa04510), metabolism of xenobiotics by cytochrome P450 (hsa00980) and ECM-receptor interaction (hsa04512) were all enriched in the two methods used to assess pathway enrichment. Based on these findings, we suggest that the genes of the primary immunodeficiency 
pathway, focal adhesion, ECM-receptor interaction and the metabolism of xenobiotics by cytochrome P450 (hsa00980) are potentially important therapeutic targets for gastric cancer. These pathways are discussed below in greater detail.

Primary immunodeficiencies are a heterogeneous group of disorders that affect cellular and humoral immunity or non-specific host defense mechanisms mediated by complement proteins and by cells such as phagocytes and natural killer cells. These immune system disorders cause increased susceptibility to malignancy. For example, patients with common variable immunodeficiency, the second most prevalent primary immunodeficiency in adults, have a 10-fold increased risk of gastric cancer (Dhalla et al., 2011). Patients in advanced stages of gastric cancer frequently suffer from cell-mediated immunodeficiency, such as the inhibition of interleukin- 2 production, the main cytokine that modulates the cell-mediated immune response, and a decrease in the total and T lymphocyte counts (Romano et al., 2003). In addition, the absolute number of T-regulatory lymphocytes (Tregs; CD4+CD25+Foxp3+) is significantly lower in gastric cancer patients than in normal individuals (Szczepanik et al., 2011).

Disorders in the regulation of humoral immunity also have a significant effect on the development of gastric cancer. For example, a significant increase in IgG Fc fucosylation has been observed in stages II and III of gastric cancer (Kodar et al., 2012). The widespread expression of CD40, a member of the tumor necrosis factor receptor superfamily, reflects the central role of CD40 in regulating humoral immunity and host defense. The stimulation of CD40 in gastric carcinoma makes cells less vulnerable to apoptosis induced by Fas or chemotherapy and increases cell motility (Yamaguchi et al., 2003).

ZAP70 (zeta-chain (TCR) associated protein kinase $70 \mathrm{kDa}$ ), IGLL1 (immunoglobulin lambda-like polypeptide 1) and CD79A (CD79a molecule, immunoglobulinassociated alpha) were enriched in the primary immunodeficiency pathway. ZAP70 may be involved in T-cellmediated immunodeficiency. ZAP-70 ectopic expression leads to enhanced B cell receptor signaling after IgM stimulation and increased expression of CCR7 (chemokine [C-C motif] receptor 7), predominantly via ERK1/2, thereby enhancing the response to and migration towards CCL21 (chemokine [C-C motif] ligand 21). In addition, cellular subsets with high ZAP-70 expression in chronic lymphocytic leukemia show increased expression of adhesion molecules and chemokine receptors (Calpe et al., 2011). IGLL1 and CD79A are associated with B cell-mediated immunodeficiency. Mutations in IGLL1 and CD79a can result in B cell deficiency and few or no $\gamma$-globulins or antibodies are produced (Storlazzi et al., 2002; Wang et al., 2002). These alterations may promote the metastasis of gastric cancer since an anti-Wnt5a antibody suppresses the Wnt5a-dependent internalization of receptors. This in turn prevents the metastasis of gastric cancer cells by inhibiting the activation of Rac1 (ras-related $\mathrm{C} 3$ botulinum toxin substrate 1 [rho family, small GTP binding protein Rac1]) and the expression of laminin $\gamma 2$ (Hanaki et al., 2012). Based on these findings, we conclude that the primary immunodeficiency pathway may affect the progress of gastric cancer by inhibiting $\mathrm{T}$ lymphocyte proliferation and antibody production by B lymphocytes, or by enhancing the expression of adhesion molecules and chemokine receptors.

The interaction between tumor cells and extracellular matrix (ECM) components such as laminin, fibronectin and collagen, has a crucial role in tumor invasion and metastasis. This interaction is facilitated by adhesion receptors such as integrins. Consequently, ECM-receptor interactions and the focal adhesion pathway may be involved in cancer metastasis. Collagen is the major constituent of the tumor ECM and several types of collagens have been implicated in the focal adhesion and ECM-receptor interaction pathways in gastric carcinoma (Yin et al., 2009). Watanabe et al. (1995) reported greater deposition of type III collagen at the periphery of poorly differentiated gastric cancer tissue compared with more central locations.

Microarray studies have shown the enhanced expression of several collagen genes (COL1A1, 1A2, 3A1, 4A1, $4 \mathrm{~A} 2,4 \mathrm{~A} 6,5 \mathrm{~A} 2,6 \mathrm{~A} 3,7 \mathrm{~A} 1,9 \mathrm{~A} 3,11 \mathrm{~A} 1$ and $18 \mathrm{~A} 1)$ in the endothelium of gastric cancer tissue compared with normal endothelium (Hippo et al., 2002; Oue et al., 2004). The most up-regulated genes in gastric cancer, such as COL1A1, 1A2, 3A1, 4A1 and 4A2, are associated with cell adhesion or migration and the ECM. COL4A6, 6A3, 17A1 and 18A1 are also associated with cell adhesion, COL1A1 with cell growth and/or maintenance, and COL1A2 and 6A3 with the 'ECM-receptor interaction' pathway (Yasui et al., 2004). In agreement with previous studies, COL6A3, $3 \mathrm{~A} 1$ and $1 \mathrm{~A} 1$ were found to be involved in focal adhesion and ECM-receptor interaction pathways. These findings suggest that targeting these genes with RNA interference could decrease the collagen content of the ECM in gastric carcinoma and reduce cell proliferation and migration. This diversity of collagens suggests that each type is associated with some aspect of gastric cancer. The identification of collagens as potential therapeutic targets will require a more complete understanding of their expression and interactions in gastric cancer.

Cytochromes P450 (CYP) are a multi-gene family of constitutive and inducible heme-containing enzymes with a crucial role in the metabolism of xenobiotics, including many potential carcinogens and various anti-cancer drugs. CYP P450s have a central role in chemical carcinogenesis and are involved in tumor initiation and promotion because they can activate or deactivate most carcinogens. Furthermore, CYP P450s can influence the response of established tumors to anti-cancer drugs by metabolizing these drugs in tumor cells (Ding and Kaminsky, 2003). The expression of major isoforms of P450, such as CYPIA and CYP3A, is en- 
hanced in gastric cancer, with CYP1A being enhanced in $51 \%$ of cases and CYP3A in 28\% (Murray et al., 1998). CYP2C9, CYP3A7 and CYP3A5 that participate in drug metabolism are down-regulated in gastric cancer. In Helicobacter pylori-positive Japanese, poor metabolizers via CYP2C19 have an increased risk of developing gastric cancer, especially the diffuse type (Sugimoto et al., 2005). In Chinese with gastric cancer the frequency of poor metabolizers via CYP2C19 is $31.8 \%$ (Shi and Chen, 2004).

As shown here, CYP2C18 and CYP2C9 were associated with the development of gastric cancer through the metabolism of xenobiotics by cytochrome P450, arachidonic acid metabolism, retinol metabolism and the linoleic acid metabolism pathway. CYP2C9 is one of the predominant epoxygenase isoforms involved in the metabolism of arachidonic acid into 12-epoxyeicosatrienoic acid (EEF). CYP2C9 epoxygenases are upregulated in human tumors and promote tumor progression and metastasis (Xu et al., 2011). Retinol may influence gastric carcinogenesis through its essential role in controlling cell proliferation and differentiation. High intakes of retinol from foods or a combination of foods and supplements are associated with a lower risk of gastric cancer (Larsson et al., 2007). CYP2C18 and CYP2C9 are related to retinol metabolism in human through their ability to transform retinol into 4OH-retinoic acid and 18-OH-retinoic acid (Marill et al., 2000). These all-trans-retinoic acids are associated with G0/G1 phase arrest and decreased VEGF expression in human gastric cancer cell lines (Zhang et al., 2007). However, dietary linoleic acid stimulates the invasion and peritoneal metastasis of gastric carcinoma cells through COX-catalyzed metabolism and the activation of ERK (Matsuoka et al., 2010). CYP2C9 is involved in linoleic acid epoxygenation and the major product of this reaction is leukotoxin that increases oxidative stress and subsequent proinflammatory events (Viswanathan et al., 2003), leading to tumor cell progression. We therefore suggest that P450 family genes are involved in gastric cancer by metabolizing exogenous anti-cancer drugs, stimulating arachidonic acid and linoleic acid metabolism and inhibiting retinol metabolism.

In conclusion, the results described here show that changes in the primary immunodeficiency pathway, focal adhesion, ECM-receptor interactions and the metabolism of xenobiotics by cytochrome $\mathrm{P} 450$ may be associated with gastric cancer. A number of candidate genes (ZAP70, IGLL1, CD79A, COL6A3, COL3A1, COL1A1, CYP2C18 and $\mathrm{CYP} 2 \mathrm{C} 9$ ) that may be involved in gastric cancer were also identified. Overall, these findings shed new light on the biology of gastric cancer and indicate new avenues for future research.

\section{Acknowledgments}

This work was supported by the Anhui Provincial Natural Science Funding for Key Projects in 2010 (grant no. KJ2010A171) and the Medicine Research Projects Schedule of the Province Health Bureau in 2009 (grant no. 09C157).

\section{References}

Altaf-Ul-Amin M, Shinbo Y, Mihara K, Kurokawa K and Kanaya S (2006) Development and implementation of an algorithm for detection of protein complexes in large interaction networks. BMC Bioinformatics 7:e207.

Calpe E, Codony C, Baptista MJ, Abrisqueta P, Carpio C, Purroy N, Bosch F and Crespo M (2011) ZAP-70 enhances migration of malignant B lymphocytes toward CCL21 by inducing CCR7 expression via IgM-ERK1/2 activation. Blood 118:4401-4410.

Chatr-aryamontri A, Ceol A, Palazzi LM, Nardelli G, Schneider MV, Castagnoli L and Cesareni G (2007) MINT: The Molecular INTeraction database. Nucleic Acids Res 35(Database issue):D572-D574.

Clements WM, Wang J, Sarnaik A, Kim OJ, MacDonald J, Fenoglio-Preiser C, Groden J and Lowy AM (2002) $\beta$-catenin mutation is a frequent cause of Wnt pathway activation in gastric cancer. Cancer Res 62:3503-3506.

Dhalla F, da Silva SP, Lucas M, Travis S and Chapel H (2011) Review of gastric cancer risk factors in patients with common variable immunodeficiency disorders, resulting in a proposal for a surveillance programme. Clin Exp Immunol 165:1-7.

Ding X and Kaminsky LS (2003) Human extrahepatic cytochromes P450: Function in xenobiotic metabolism and tissue-selective chemical toxicity in the respiratory and gastrointestinal tracts. Annu Rev Pharmacol Toxicol 43:149-173.

D'Souza M, Zhu X and Frisina RD (2008) Novel approach to select genes from RMA normalized microarray data using functional hearing tests in aging mice. J Neurosci Methods 171:279-287.

D’Ugo D, Rausei S, Biondi A and Persiani R (2009) Preoperative treatment and surgery in gastric cancer: Friends or foes? Lancet Oncol 10:191-195.

Fukushima A, Kusano M, Redestig H, Arita M and Saito K (2011) Metabolomic correlation-network modules in Arabidopsis based on a graph-clustering approach. BMC Syst Biol 5:e1.

Gentleman RC, Carey VJ, Bates DM, Bolstad B, Dettling M, Dudoit S, Ellis B, Gautier L, Ge Y, Gentry J, et al. (2004) Bioconductor: Open software development for computational biology and bioinformatics. Genome Biol 5:R80.

Hanaki H, Yamamoto H, Sakane H, Matsumoto S, Ohdan H, Sato A and Kikuchi A (2012) An anti-Wnt5a antibody suppresses metastasis of gastric cancer cells in vivo by inhibiting receptor-mediated endocytosis. Mol Cancer Ther 11:298-307.

Hippo Y, Taniguchi H, Tsutsumi S, Machida N, Chong JM, Fukayama M, Kodama T and Aburatani H (2002) Global gene expression analysis of gastric cancer by oligonucleotide microarrays. Cancer Res 62:233-240.

Huang da W, Sherman BT and Lempicki RA (2008) Systematic and integrative analysis of large gene lists using DAVID bioinformatics resources. Nat Protoc 4:44-57.

Hwang S, Son SW, Kim SC, Kim YJ, Jeong H and Lee D (2008) A protein interaction network associated with asthma. J Theor Biol 252:722-731. 
Kanai M, Konda Y, Nakajima T, Izumi Y, Kanda N, Nanakin A, Kubohara Y and Chiba T (2003) Differentiation-inducing factor-1 (DIF-1) inhibits STAT3 activity involved in gastric cancer cell proliferation via MEK-ERK-dependent pathway. Oncogene 22:548-554.

Kanehisa M (2002) The KEGG database. Novartis Found Symp 247:91-101 (and discussion 101-103, 119-128, 244-252).

Kang MH, Kim JS, Seo JE, Oh SC and Yoo YA (2010) BMP2 accelerates the motility and invasiveness of gastric cancer cells via activation of the phosphatidylinositol 3-kinase (PI3K)/Akt pathway. Exp Cell Res 316:24-37.

Keshava Prasad TS, Goel R, Kandasamy K, Keerthikumar S, Kumar S, Mathivanan S, Telikicherla D, Raju R, Shafreen B, Venugopal A, et al. (2009) Human protein reference database - 2009 update. Nucleic Acids Res 37(Suppl 1):D767D772.

Kim SS, Shetty K, Katuri V, Kitisin K, Baek HJ, Tang Y, Marshall B, Johnson L, Mishra B and Mishra L (2006) TGF- $\beta$ signaling pathway inactivation and cell cycle deregulation in the development of gastric cancer: Role of the beta-spectrin, ELF. Biochem Biophys Res Commun 344:1216-1223.

Kodar K, Stadlmann J, Klaamas K, Sergeyev B and Kurtenkov O (2012) Immunoglobulin G Fc N-glycan profiling in patients with gastric cancer by LC-ESI-MS: Relation to tumor progression and survival. Glycoconj J 29:57-66.

Kurayoshi M, Oue N, Yamamoto H, Kishida M, Inoue A, Asahara T, Yasui W and Kikuchi A (2006) Expression of Wnt-5a is correlated with aggressiveness of gastric cancer by stimulating cell migration and invasion. Cancer Res 66:1043910448.

Larsson SC, Bergkvist L, Naslund I, Rutegard J and Wolk A (2007) Vitamin A, retinol, and carotenoids and the risk of gastric cancer: A prospective cohort study. Am J Clin Nutr 85:497-503.

Lin MT, Lin BR, Chang CC, Chu CY, Su HJ, Chen ST, Jeng YM and Kuo ML (2007a) IL-6 induces AGS gastric cancer cell invasion via activation of the c-Src/RhoA/ROCK signaling pathway. Int J Cancer 120:2600-2608.

Lin HL, Yang MH, Wu CW, Chen PM, Yang YP, Chu YR, Kao $\mathrm{CL}, \mathrm{Ku} \mathrm{HH}$, Lo JF, Liou JP, et al. (2007b) 2-Methoxyestradiol attenuates phosphatidylinositol 3-kinase/Akt pathway-mediated metastasis of gastric cancer. Int $\mathrm{J}$ Cancer 121:2547-2555.

Liu ZP, Wang Y, Zhang XS and Chen L (2010) Identifying dysfunctional crosstalk of pathways in various regions of Alzheimer's disease brains. BMC Syst Biol 4(Suppl 2):S11.

Ma X, Chen K, Huang S, Zhang X, Adegboyega PA, Evers BM, Zhang $\mathrm{H}$ and Xie J (2005) Frequent activation of the hedgehog pathway in advanced gastric adenocarcinomas. Carcinogenesis 26:1698-1705.

Marill J, Cresteil T, Lanotte M and Chabot GG (2000) Identification of human cytochrome P450s involved in the formation of all-trans-retinoic acid principal metabolites. Mol Pharmacol 58:1341-1348.

Matsuoka T, Adair JE, Lih FB, Hsi LC, Rubino M, Eling TE, Tomer KB, Yashiro M, Hirakawa K, Olden K, et al. (2010) Elevated dietary linoleic acid increases gastric carcinoma cell invasion and metastasis in mice. Br J Cancer 103:11821191.
Murray GI, Taylor MC, Burke MD and Melvin WT (1998) Enhanced expression of cytochrome P450 in stomach cancer. Br J Cancer 77:1040-1044.

Offerhaus GJ, Giardiello FM, Krush AJ, Booker SV, Tersmette AC, Kelley NC and Hamilton SR (1992) The risk of upper gastrointestinal cancer in familial adenomatous polyposis. Gastroenterology 102:1980-1982.

Oue N, Hamai Y, Mitani Y, Matsumura S, Oshimo Y, Aung PP, Kuraoka K, Nakayama H and Yasui W (2004) Gene expression profile of gastric carcinoma. Cancer Res 64:2397-2405.

Peng L, Ran YL, Hu H, Yu L, Liu Q, Zhou Z, Sun YM, Sun LC, Pan J, Sun LX, et al. (2009) Secreted LOXL2 is a novel therapeutic target that promotes gastric cancer metastasis via the Src/FAK pathway. Carcinogenesis 30:1660-1669.

Romano F, Caprotti R, Bravo AF, Conti M, Colombo G, Piacentini G, Uggeri F Jr and Uggeri F (2003) Radical surgery does not recover immunodeficiency associated with gastric cancer. J Exp Clin Cancer Res 22:179-184.

Shi WX and Chen SQ (2004) Frequencies of poor metabolizers of cytochrome P450 2C19 in esophagus cancer, stomach cancer, lung cancer and bladder cancer in Chinese population. World J Gastroenterol 10:1961-1963.

Smyth GK (2004) Linear models and empirical Bayes methods for assessing differential expression in microarray experiments. Stat Appl Genet Mol Biol 3:Article3.

Stark C, Breitkreutz BJ, Chatr-Aryamontri A, Boucher L, Oughtred R, Livstone MS, Nixon J, Van Auken K, Wang X, Shi X, et al. (2011) The BioGRID Interaction Database: 2011 update. Nucleic Acids Res 39(Suppl 1):D698-D704.

Storlazzi CT, Specchia G, Anelli L, Albano F, Pastore D, Zagaria A, Rocchi M and Liso V (2002) Breakpoint characterization of der(9) deletions in chronic myeloid leukemia patients. Genes Chromosomes Cancer 35:271-276.

Strimmer K (2008) fdrtool: A versatile R package for estimating local and tail area-based false discovery rates. Bioinformatics 24:1461-1462.

Sugimoto M, Furuta T, Shirai N, Nakamura A, Kajimura M, Sugimura H, Hishida A and Ishizaki T (2005) Poor metabolizer genotype status of CYP2C19 is a risk factor for developing gastric cancer in Japanese patients with Helicobacter pylori infection. Aliment Pharmacol Ther 22:1033-1040.

Szczepanik AM, Siedlar M, Sierzega M, Goroszeniuk D, Bukowska-Strakova K, Czupryna A and Kulig J (2011) T-regulatory lymphocytes in peripheral blood of gastric and colorectal cancer patients. World J Gastroenterol 17:343-348.

To KF, Chan MW, Leung WK, Yu J, Tong JH, Lee TL, Chan FK and Sung JJ (2001) Alterations of frizzled (FzE3) and secreted frizzled related protein (hsFRP) expression in gastric cancer. Life Sci 70:483-489.

Viswanathan S, Hammock BD, Newman JW, Meerarani P, Toborek M and Hennig B (2003) Involvement of CYP 2C9 in mediating the proinflammatory effects of linoleic acid in vascular endothelial cells. J Am Coll Nutr 22:502-510.

Wang Y, Kanegane H, Sanal O, Tezcan I, Ersoy F, Futatani T and Miyawaki T (2002) Novel Ig $\alpha(C D 79 a)$ gene mutation in a Turkish patient with B cell-deficient agammaglobulinemia. Am J Med Genet 108:333-336.

Watanabe M, Hirano T and Asano G (1995) Roles of myofibroblasts in the stroma of human gastric carcinoma. Nippon Geka Gakkai Zasshi 96:10-18. 
Xu X, Zhang XA and Wang DW (2011) The roles of CYP450 epoxygenases and metabolites, epoxyeicosatrienoic acids, in cardiovascular and malignant diseases. Adv Drug Deliv Rev 63:597-609.

Yamaguchi H, Tanaka F, Sadanaga N, Ohta M, Inoue H and Mori M (2003) Stimulation of CD40 inhibits Fas- or chemotherapy-mediated apoptosis and increases cell motility in human gastric carcinoma cells. Int J Oncol 23:1697-1702.

Yasui W, Oue N, Ito R, Kuraoka K and Nakayama H (2004) Search for new biomarkers of gastric cancer through serial analysis of gene expression and its clinical implications. Cancer Sci 95:385-392.

Yin Y, Zhao Y, Li AQ and Si JM (2009) Collagen: A possible prediction mark for gastric cancer. Med Hypotheses 72:163165.

Yoo YA, Kang MH, Kim JS and Oh SC (2008) Sonic hedgehog signaling promotes motility and invasiveness of gastric cancer cells through TGF- $\beta$-mediated activation of the ALK5Smad 3 pathway. Carcinogenesis 29:480.

Zhang JP, Chen XY and Li JS (2007) Effects of all-trans-retinoic on human gastric cancer cells BGC-823. J Dig Dis 8:29-34.

\section{Internet Resources}

Gene Expression Omnibus (GEO) data repository at NCBI, http://www.ncbi.nlm.nih.gov/geo/GSE2685 (November 8, 2011).

R program: http://www.r-project.org/ (November 11, 2011). DPClus, http://kanaya.naist.jp/DPClus/.

\section{Supplementary Material}

The following online material is available for this article:

Table S1 - PPI data.

This material is available as part of the online article from http://www.scielo.br/gmb.

Associate Editor: Carlos F.M. Menck

License information: This is an open-access article distributed under the terms of the Creative Commons Attribution License, which permits unrestricted use, distribution, and reproduction in any medium, provided the original work is properly cited. 


\begin{tabular}{|c|c|}
\hline Table S1 & \\
\hline Protein1 & Protein2 \\
\hline ARF1 & GGA3 \\
\hline MYC & SMAD2 \\
\hline SALL1 & TERF1 \\
\hline KHDRBS1 & RAPSN \\
\hline CD151 & ITGB1 \\
\hline PTH1R & SLC9A3R2 \\
\hline EPOR & PTPN11 \\
\hline CR1 & CR2 \\
\hline JUN & RB1 \\
\hline COL1A1 & NID1 \\
\hline BAK1 & VDAC1 \\
\hline NFKB1 & RXRA \\
\hline LILRB4 & PTPN6 \\
\hline CFL1 & HSPH1 \\
\hline CALCR & CALM1 \\
\hline PRLR & VAV1 \\
\hline EP300 & IRF1 \\
\hline BRCA2 & STAT5A \\
\hline FN1 & NOV \\
\hline $\mathrm{CCNH}$ & CDK7 \\
\hline TESK1 & YWHAB \\
\hline EP300 & TP53 \\
\hline $\mathrm{EZH} 2$ & SUZ12 \\
\hline GNG4 & RAF1 \\
\hline RELB & SMARCA4 \\
\hline CTNNB1 & SMAD2 \\
\hline RYR2 & SMAD5 \\
\hline HSPH1 & PGK1 \\
\hline SPG7 & TRIM28 \\
\hline FYN & MCAM \\
\hline NEFL & TSC1 \\
\hline LAPTM5 & NEDD4 \\
\hline UBE2B & UNC119 \\
\hline EXT2 & TRAP1 \\
\hline MATN1 & MATN3 \\
\hline KCNJ12 & SNTB2 \\
\hline TAF1C & TP53 \\
\hline $\mathrm{CDC} 25 \mathrm{C}$ & PLK3 \\
\hline FANCC & STAT1 \\
\hline GRIN2A & IL16 \\
\hline ESR1 & ZBTB16 \\
\hline LMO2 & MAPRE2 \\
\hline APCS & CRP \\
\hline HNRNPK & WWOX \\
\hline STAT1 & VDR \\
\hline
\end{tabular}




\begin{tabular}{|c|c|}
\hline STOM & STOM \\
\hline YAP1 & YWHAQ \\
\hline$A R$ & SP1 \\
\hline PURA & SP1 \\
\hline PFN1 & UNC119 \\
\hline MAX & MNT \\
\hline OCLN & TJP2 \\
\hline CLDN5 & TJP1 \\
\hline CDK4 & SETDB1 \\
\hline IRF2 & NFKB1 \\
\hline CDC25A & YWHAZ \\
\hline GRB2 & MAP4K5 \\
\hline CRK & MAP4K1 \\
\hline GJA1 & S100A1 \\
\hline CGA & PTPN12 \\
\hline MYOD1 & RB1 \\
\hline ATM & H2AFX \\
\hline IER3 & PPP2R5C \\
\hline ACVR2A & ENG \\
\hline RPA1 & RPA1 \\
\hline CDC6 & PPP2R3A \\
\hline HDAC2 & MAD1L1 \\
\hline RCOR1 & REST \\
\hline NR5A1 & NRIP1 \\
\hline PSMD4 & PSMD6 \\
\hline KIT & PLCG1 \\
\hline RPS6KB1 & TERT \\
\hline $\mathrm{HCK}$ & RASA1 \\
\hline CENPC1 & UBTF \\
\hline PDGFRB & RAF1 \\
\hline DMD & KCNJ12 \\
\hline PNN & PRPF4B \\
\hline CSNK2A1 & UBTF \\
\hline $\mathrm{DHX9}$ & JUN \\
\hline LMO3 & MDFI \\
\hline MYOG & TCF3 \\
\hline POLR2E & POLR2E \\
\hline HNF4A & SP1 \\
\hline COPS5 & TP53 \\
\hline HDAC2 & SNW1 \\
\hline IL2RG & SHB \\
\hline CD19 & VAV1 \\
\hline E2F1 & RB1 \\
\hline KLKB1 & KNG1 \\
\hline AKAP5 & IQGAP1 \\
\hline GNB2L1 & PABPC1 \\
\hline MAP2K2 & MAP2K2 \\
\hline
\end{tabular}




\begin{tabular}{|c|c|}
\hline EP300 & SMAD7 \\
\hline IRX4 & RARA \\
\hline DGKZ & PLCG1 \\
\hline ATXN1 & BTG3 \\
\hline ATF1 & BRCA1 \\
\hline ARL4D & DNAJA1 \\
\hline IRF4 & STAT6 \\
\hline FYB & WAS \\
\hline MCM6 & ORC2L \\
\hline PRKDC & RPA2 \\
\hline ATXN3 & EWSR1 \\
\hline KPNA2 & SGK1 \\
\hline SNTA1 & XRCC6 \\
\hline ITPR1 & TRPC3 \\
\hline DVL1 & SMAD7 \\
\hline AGER & S100P \\
\hline BCL3 & JUN \\
\hline CASP6 & TRAF1 \\
\hline MDFI & SLC35A2 \\
\hline LRP2 & SCGB1A1 \\
\hline $\mathrm{GCSH}$ & NMI \\
\hline KHDRBS1 & SRC \\
\hline TOP2B & TOPBP1 \\
\hline RDH5 & RLBP1 \\
\hline NKX2 & PAX8 \\
\hline PRKCE & VDAC1 \\
\hline BIRC2 & MAGEA11 \\
\hline DAG1 & DAG1 \\
\hline EEA1 & RAB5A \\
\hline PDGFRB & RASA1 \\
\hline MAPK1 & TPR \\
\hline BAD & MCL1 \\
\hline KTN1 & RHOA \\
\hline CIITA & NCOA1 \\
\hline ACVR2A & BMP6 \\
\hline DNAJB1 & HSF1 \\
\hline CRMP1 & PPP1R8 \\
\hline CD36 & ITGB3 \\
\hline USF1 & USF1 \\
\hline ATF4 & GTF2F2 \\
\hline APP & NCSTN \\
\hline UBA52 & UBA52 \\
\hline NEB & TMOD1 \\
\hline THRB & TRIP4 \\
\hline CALM1 & TCF4 \\
\hline COL4A1 & NID1 \\
\hline FBLN2 & PRELP \\
\hline
\end{tabular}




\begin{tabular}{|c|c|}
\hline BAT2 & PRMT2 \\
\hline GRB2 & PLCG1 \\
\hline EWSR1 & USP7 \\
\hline KLK2 & SERPINA5 \\
\hline ERH & SH3GL2 \\
\hline GCM1 & HDAC1 \\
\hline GNB2L1 & TP73 \\
\hline ESRRA & LYST \\
\hline KRT18 & TRADD \\
\hline NCOA1 & STAT6 \\
\hline COIL & TAF9 \\
\hline ARL1 & GOLGA1 \\
\hline ITGA4 & LGALS8 \\
\hline PSMB5 & PSMB7 \\
\hline BCL6 & IRF4 \\
\hline PAPPA & PLG \\
\hline CDC6 & MYC \\
\hline PDLIM7 & SPP1 \\
\hline KPNB1 & SMAD2 \\
\hline CYC1 & CYC1 \\
\hline ETS1 & POU1F1 \\
\hline GMFB & MAPK14 \\
\hline NROB1 & SREBF1 \\
\hline KLF4 & SP1 \\
\hline BRAF & RAP1GAP \\
\hline SMN1 & SNRPD1 \\
\hline ATXN2L & MPL \\
\hline ABL1 & ADAM15 \\
\hline KDR & PLCG1 \\
\hline$A 2 M$ & B2M \\
\hline LCK & PTPN6 \\
\hline COMT & TRIP13 \\
\hline GRB2 & ITGA2B \\
\hline DNAJB1 & HSPA1A \\
\hline CD81 & CD9 \\
\hline BRCA1 & FANCA \\
\hline CHMP1A & USP8 \\
\hline CDK5 & PAK1 \\
\hline TRIP13 & TRIP13 \\
\hline EP300 & ETS2 \\
\hline SHB & VAV1 \\
\hline FBLN2 & FN1 \\
\hline HDAC1 & RELA \\
\hline BAD & $\mathrm{BCL} 2$ \\
\hline GJA1 & GJA5 \\
\hline CHD4 & HDAC1 \\
\hline CDH18 & CDH6 \\
\hline
\end{tabular}




\begin{tabular}{|c|c|}
\hline RAD52 & RAD52 \\
\hline IRF7 & MYD88 \\
\hline IDE & IGF1 \\
\hline CD22 & PTPRC \\
\hline CSTF2 & DDX1 \\
\hline PLCG1 & VAV1 \\
\hline KRT20 & VIM \\
\hline TEAD1 & VGLL4 \\
\hline CREBBP & СТВP1 \\
\hline CALR & LRP1 \\
\hline MYH10 & PBX1 \\
\hline BRCA1 & CSNK2A \\
\hline ATF6 & NFYC \\
\hline KPNB1 & LYST \\
\hline TGFB1 & VTN \\
\hline S100A1 & S100B \\
\hline RBP4 & TTR \\
\hline GSTZ1 & GSTZ1 \\
\hline CHAF1A & CHAF1B \\
\hline MAPK1 & PTPN5 \\
\hline FXR2 & RBPMS \\
\hline BTK & PRKCB \\
\hline IGFALS & IGFBP5 \\
\hline BMP6 & BMPR1A \\
\hline CD4 & LCK \\
\hline ATXN1 & SREBF1 \\
\hline TRAF6 & UBE2N \\
\hline ACP1 & ZAP70 \\
\hline HMGB1 & PLAT \\
\hline HRAS & PIK3CD \\
\hline EGFR & ERBB3 \\
\hline CACNA1S & SRI \\
\hline FAS & HIPK3 \\
\hline E4F1 & RB1 \\
\hline KHDRBS1 & LCK \\
\hline $\mathrm{CDX} 2$ & CREBBP \\
\hline ARHGDIG & $\mathrm{RHOH}$ \\
\hline AIP & PPARA \\
\hline EP300 & TWIST1 \\
\hline CCDC85B & PBXIP1 \\
\hline ATR & TP53 \\
\hline FLT1 & SHC1 \\
\hline FTL & PTN \\
\hline RAB27A & ZBTB16 \\
\hline ERBB3 & SHC1 \\
\hline ADAM15 & GRB2 \\
\hline ERBB2 & SHC1 \\
\hline
\end{tabular}




\begin{tabular}{|c|c|}
\hline $\mathrm{BMX}$ & SRC \\
\hline CDC25B & CSNK $2 A 1$ \\
\hline DDR1 & TM4SF1 \\
\hline SPRR2C & USP13 \\
\hline CEBPA & NCOA6 \\
\hline NCOA1 & TRIP4 \\
\hline BAD & PIM2 \\
\hline RUNX3 & TLE1 \\
\hline KRAS & RALGDS \\
\hline COPS5 & COPS8 \\
\hline PLG & SPARC \\
\hline ASCL1 & EP300 \\
\hline TNFAIP3 & YWHAH \\
\hline PAPOLA & SMAD2 \\
\hline CXCL5 & MMP9 \\
\hline ABL1 & TP73 \\
\hline ITGB1 & NME1 \\
\hline ELN & FCN1 \\
\hline KPNA3 & NFKB1 \\
\hline FXR2 & PSME3 \\
\hline NFKB1 & PARP1 \\
\hline NEUROG1 & SMAD1 \\
\hline FN1 & ITGB7 \\
\hline SAT1 & UNC119 \\
\hline NEDD4 & SCNN1A \\
\hline ACTN1 & TTN \\
\hline CSNK1E & DVL1 \\
\hline CD44 & IGFBP3 \\
\hline TNFAIP3 & TRAF1 \\
\hline SKP1 & SKP1 \\
\hline REL & TBP \\
\hline CIITA & XPO1 \\
\hline IRS1 & SHC1 \\
\hline C1QBP & GAB1 \\
\hline CD46 & DLG4 \\
\hline IL2RB & SHB \\
\hline MAР3К11 & MAP4K1 \\
\hline CASR & CASR \\
\hline BRCA1 & RFC1 \\
\hline ORC1L & SKP2 \\
\hline MAGEA11 & ZBTB16 \\
\hline SP1 & TAL1 \\
\hline CREB1 & KAT5 \\
\hline RPA2 & RPLP1 \\
\hline ATXN1 & ATXN1 \\
\hline CES1 & CES1 \\
\hline FGFR1 & GRB14 \\
\hline
\end{tabular}




\begin{tabular}{|c|c|}
\hline ARF6 & PLD1 \\
\hline CSK & SRC \\
\hline HSPG2 & PDGFB \\
\hline GNAS & TTC1 \\
\hline ACP5 & MAPK9 \\
\hline CDC25B & SFN \\
\hline ARID4A & HDAC1 \\
\hline CCL5 & DPP4 \\
\hline RBBP8 & RBBP8 \\
\hline PPFIA1 & PPP2R5D \\
\hline S100A8 & TP53 \\
\hline SEPHS1 & SEPHS1 \\
\hline CAV1 & SCP2 \\
\hline ABL1 & SLC9A2 \\
\hline MGMT & PRKCA \\
\hline CSNK2A2 & FGF2 \\
\hline MYB & SND1 \\
\hline CREBBP & CUX1 \\
\hline RRM2 & TP53 \\
\hline APOA1 & APOF \\
\hline$A R$ & RNF4 \\
\hline OSTF1 & SMN1 \\
\hline HMGB1 & TBP \\
\hline CD19 & CD81 \\
\hline HTT & TAF4 \\
\hline PSMC1 & PSMD7 \\
\hline SH3BP2 & YWHAQ \\
\hline MDM2 & TP73 \\
\hline ATM & RHEB \\
\hline$A R$ & ETV5 \\
\hline CRK & GRB2 \\
\hline GNAQ & GRM7 \\
\hline GNAI2 & TTC1 \\
\hline UGP2 & UGP2 \\
\hline IRF9 & STAT1 \\
\hline ACTA1 & TAGLN \\
\hline AKT1 & FOXO1 \\
\hline CBL & INPPL1 \\
\hline CSDA & GSK3B \\
\hline CANX & SLC4A1 \\
\hline PDE6G & SRC \\
\hline CDKN1A & MAРК8 \\
\hline SCN5A & SNTB1 \\
\hline TGFB1 & TGFBR3 \\
\hline ITGB1 & PXN \\
\hline CALM1 & CRHR1 \\
\hline DCTN1 & RAB6A \\
\hline
\end{tabular}




\begin{tabular}{|c|c|}
\hline DUSP5 & MAPK3 \\
\hline CUX1 & SATB1 \\
\hline FYN & SLAMF1 \\
\hline SRC & STAT3 \\
\hline FYN & PECAM1 \\
\hline SNRPD2 & SNRPF \\
\hline FLNC & MAP2K4 \\
\hline MAP2K4 & МАРЗК4 \\
\hline DRAP1 & TK1 \\
\hline PNN & SRRM2 \\
\hline CXCL11 & DPP4 \\
\hline ACTN1 & ZYX \\
\hline PDHA1 & PDHB \\
\hline CSF1 & CSF1R \\
\hline MRPL28 & TUBB3 \\
\hline MAPK8 & PRKDC \\
\hline KPNB1 & TERF1 \\
\hline TBCB & USP7 \\
\hline MYOD1 & SP1 \\
\hline IRF1 & STAT1 \\
\hline ATN1 & VIM \\
\hline DLG3 & ERBB4 \\
\hline GNAI3 & RGS16 \\
\hline FCGR2A & SYK \\
\hline ОТС & TOMM20 \\
\hline SMAD2 & SMAD4 \\
\hline CAT & PTPN11 \\
\hline $\mathrm{HCK}$ & IL6ST \\
\hline NGF & NGFR \\
\hline MDM2 & RB1 \\
\hline SMARCA4 & TP53 \\
\hline TSC1 & YWHAZ \\
\hline CD81 & ZBTB16 \\
\hline RBPMS & RBPMS \\
\hline$A R$ & $\mathrm{AR}$ \\
\hline POLR2C & POLR2G \\
\hline BRCA1 & MYC \\
\hline KAT5 & STAT3 \\
\hline GRIN2C & IL16 \\
\hline E4F1 & HDAC1 \\
\hline BSG & PDLIM7 \\
\hline MSX2 & XRCC6 \\
\hline JAK3 & STAM \\
\hline ESR1 & FLII \\
\hline EXT1 & EXT1 \\
\hline CHML & RAB5A \\
\hline ATXN1 & DAZAP2 \\
\hline
\end{tabular}




\begin{tabular}{|c|c|}
\hline SMAD4 & TOB1 \\
\hline ACTB & ACTG1 \\
\hline MMP2 & TIMP4 \\
\hline ANXA1 & KRT8 \\
\hline EP300 & GATA6 \\
\hline GSK3B & TP53 \\
\hline SMAD4 & SNRNP70 \\
\hline ATN1 & ATN1 \\
\hline TCF3 & TCF3 \\
\hline BRCA1 & TOP2A \\
\hline LMNA & LMNB1 \\
\hline NR3C1 & RELA \\
\hline EP300 & TCF3 \\
\hline HGF & SDC2 \\
\hline CTBP1 & CTBP1 \\
\hline AMPH & CDK5R1 \\
\hline HSP90AB1 & PPID \\
\hline CREBBP & TCF3 \\
\hline MAР3К8 & REL \\
\hline NFKB1 & NR3C1 \\
\hline BRCA1 & CREB1 \\
\hline CRKL & RAPGEF1 \\
\hline CSNK1A1 & HMGB2 \\
\hline PPP1CC & SMARCB1 \\
\hline NFKB2 & TSC22D3 \\
\hline EGR1 & NAB2 \\
\hline CTSB & MARCKS \\
\hline EWSR1 & ZNF165 \\
\hline ESR1 & XBP1 \\
\hline SMAD5 & U2AF2 \\
\hline HDAC2 & RBBP4 \\
\hline TP53BP2 & YAP1 \\
\hline FXR2 & RBBP8 \\
\hline HDAC1 & HDAC1 \\
\hline HOXA9 & RBPMS \\
\hline EGFR & TGFA \\
\hline CTLA4 & FYN \\
\hline PFN2 & VASP \\
\hline TERF2 & XRCC6 \\
\hline BRF1 & CSNK2A1 \\
\hline NR6A1 & NR6A1 \\
\hline ARNTL & CLOCK \\
\hline CEBPD & CREBBP \\
\hline SLC6A1 & STX1A \\
\hline CFTR & EZR \\
\hline $\mathrm{ERH}$ & TLE1 \\
\hline PTPN6 & SYK \\
\hline
\end{tabular}




\begin{tabular}{|c|c|}
\hline CD151 & ITGA3 \\
\hline CRMP1 & DPYSL2 \\
\hline ANXA5 & FDFT1 \\
\hline FADD & PEA15 \\
\hline MAP3K14 & RPS11 \\
\hline CDC25A & SMAD3 \\
\hline IL5 & IL5RA \\
\hline DVL1 & SMAD2 \\
\hline CCDC85B & FXR2 \\
\hline CA2 & NONO \\
\hline AR & NROB2 \\
\hline LRP1 & SHC1 \\
\hline AKAP13 & PRKAR2A \\
\hline CHRNA1 & CHRNG \\
\hline FEZ1 & P4HB \\
\hline MSN & SELPLG \\
\hline SIAH1 & SYP \\
\hline CRK & PXN \\
\hline ABL1 & ZAP70 \\
\hline FEZ2 & PRKCZ \\
\hline COPB2 & RGS2 \\
\hline APP & KLC1 \\
\hline LCK & PRKCQ \\
\hline OGG1 & PRKCA \\
\hline RND3 & SFN \\
\hline ATXN2 & $\mathrm{CHGB}$ \\
\hline MAPK1 & STAT5A \\
\hline CSNK2A1 & HIF1A \\
\hline IRAK1 & ITGAM \\
\hline GAB1 & МАРЗКЗ \\
\hline СТВP1 & RBBP8 \\
\hline NR3C1 & SMARCC1 \\
\hline ACR & SERPINA5 \\
\hline СCT7 & TCP1 \\
\hline MUC1 & PRKCD \\
\hline ILK & PXN \\
\hline VBP1 & VHL \\
\hline APBA1 & HTR2C \\
\hline DLG4 & GRIN2C \\
\hline ACTG1 & TMSB4X \\
\hline NCOR2 & THRB \\
\hline NCOA1 & THRA \\
\hline AHR & HSP90AA1 \\
\hline CANX & CFTR \\
\hline RALGDS & RAP1A \\
\hline EWSR1 & GNPDA1 \\
\hline CRADD & EEF1A1 \\
\hline
\end{tabular}




\begin{tabular}{|c|c|}
\hline BMPR1A & BMPR2 \\
\hline RBL2 & SNW1 \\
\hline ITGB3 & PDGFRB \\
\hline TP53 & WWOX \\
\hline AKAP9 & PRKAR2A \\
\hline FUBP1 & GTF2H1 \\
\hline PLOD2 & PLOD2 \\
\hline CDH3 & CTNNA1 \\
\hline DAZ1 & DAZAP2 \\
\hline EIF3B & EIF4G1 \\
\hline LYN & TEC \\
\hline BAT3 & PRNP \\
\hline MSN & VCAM1 \\
\hline HAX1 & IL1A \\
\hline HDAC2 & MXD1 \\
\hline FEZ1 & FEZ1 \\
\hline HSP90AB1 & МАР3К14 \\
\hline FGF1 & HSPA9 \\
\hline NF2 & SDCBP \\
\hline CBLB & NEDD4 \\
\hline MDM2 & MED1 \\
\hline GRB2 & RAPGEF1 \\
\hline AKAP1 & PRKAR1A \\
\hline HNRNPU & POU3F4 \\
\hline PDAP1 & PDGFB \\
\hline ACTA1 & DMD \\
\hline EWSR1 & PDHX \\
\hline RING1 & RNF2 \\
\hline HSPA9 & MVD \\
\hline NR1I3 & RXRA \\
\hline CAPNS1 & TERF1 \\
\hline CLU & TGFBR1 \\
\hline PLCG1 & RHOA \\
\hline ATXN1 & FHL2 \\
\hline RPA1 & RPA4 \\
\hline ETS2 & NR3C1 \\
\hline ARR3 & $\mathrm{RHO}$ \\
\hline $\mathrm{GC}$ & $\mathrm{GC}$ \\
\hline FGA & FGG \\
\hline GJA1 & TJP1 \\
\hline FHIT & FHIT \\
\hline CA4 & SLC4A1 \\
\hline HSF4 & HSF4 \\
\hline OCRL & RAB6A \\
\hline DAPK1 & DAPK1 \\
\hline PTPN11 & STAT5B \\
\hline EEF1G & HARS \\
\hline
\end{tabular}




\begin{tabular}{|c|c|}
\hline IER3 & MAPK1 \\
\hline RFX1 & SMAD4 \\
\hline$A H R$ & AIP \\
\hline JUN & RELA \\
\hline SMARCB1 & TP53 \\
\hline NFYB & TP73 \\
\hline ETS1 & SRC \\
\hline STX1A & VAMP2 \\
\hline LCN2 & LCN2 \\
\hline HMGB2 & SET \\
\hline CDKN1A & CSNK2B \\
\hline ABLIM1 & VIM \\
\hline TAF4 & TAF5 \\
\hline MAP2K2 & MAPK1 \\
\hline SULT1E1 & TP53 \\
\hline BCL2L1 & RTN1 \\
\hline TAF15 & TAF7 \\
\hline BARD1 & KAT5 \\
\hline CLTC & MAP3K10 \\
\hline ESR1 & MED24 \\
\hline CCDC106 & CRMP1 \\
\hline MMP2 & PZP \\
\hline SDCBP & SOX4 \\
\hline PKD2 & TRPC1 \\
\hline EPHB2 & RASA1 \\
\hline PTN & TOMM20 \\
\hline RCC1 & XPO1 \\
\hline BAT3 & SMN1 \\
\hline ESR1 & ESR2 \\
\hline GNB2L1 & RB1 \\
\hline HSF1 & HSF1 \\
\hline HSP90AA1 & HSP90AA1 \\
\hline EPAS1 & VHL \\
\hline PIAS1 & SUMO1 \\
\hline ESR1 & SMAD2 \\
\hline IDE & IGF2 \\
\hline RFC2 & RFC5 \\
\hline $\mathrm{CDH} 1$ & $\mathrm{CDH} 1$ \\
\hline IGF1 & IGFBP5 \\
\hline CAV1 & HRAS \\
\hline ADORA2A & ADORA2A \\
\hline SKI & SNW1 \\
\hline GRB2 & INPP5D \\
\hline BAG1 & RB1 \\
\hline FGF1 & FGFR1 \\
\hline CDSN & CDSN \\
\hline YWHAB & YWHAE \\
\hline
\end{tabular}




\begin{tabular}{|c|c|}
\hline MAP2K1 & RAF1 \\
\hline PPP2CA & PPP2R1B \\
\hline DLG4 & KCNJ4 \\
\hline COMT & LITAF \\
\hline SRC & STAT1 \\
\hline PTH1R & PTHLH \\
\hline EP300 & STAT6 \\
\hline SRC & SRF \\
\hline RBL2 & TAF1 \\
\hline РРP3СA & RCAN2 \\
\hline CD6 & SDCBP \\
\hline MAP2K5 & MAPK7 \\
\hline LRRC41 & RBPMS \\
\hline RIN1 & YWHAZ \\
\hline EWSR1 & MAPK1IP1L \\
\hline CASP10 & FADD \\
\hline IL1A & NDN \\
\hline IGFBP3 & PLG \\
\hline CCL5 & VCAN \\
\hline ITK & LCP2 \\
\hline PAK2 & YES1 \\
\hline SLC6A3 & SNCA \\
\hline PML & RARA \\
\hline GNB2L1 & ITGB2 \\
\hline CCL5 & SDC4 \\
\hline PCNA & RFC5 \\
\hline ALB & AMBP \\
\hline GRB2 & PIK3R2 \\
\hline AKAP6 & PRKAR2A \\
\hline CCL2 & VCAN \\
\hline DNTT & PCNA \\
\hline SEC23B & SEC24C \\
\hline $\mathrm{BCL} 2$ & PPP2CA \\
\hline DPF2 & RELB \\
\hline CBLB & CRY1 \\
\hline COPS5 & SMAD4 \\
\hline SMAD6 & SMAD7 \\
\hline FYN & NTRK2 \\
\hline CCNA1 & CDKN1A \\
\hline IL5RA & SDCBP \\
\hline FASLG & FYN \\
\hline HSP90AA1 & NOS3 \\
\hline BCAT2 & BCAT2 \\
\hline CCDC85B & ZBTB16 \\
\hline DLG4 & LRP1 \\
\hline SERPINB9 & TP53 \\
\hline APLP1 & STX5 \\
\hline
\end{tabular}




\begin{tabular}{|c|c|}
\hline PFN1 & TLE1 \\
\hline NEDD9 & SMAD3 \\
\hline NFKBIE & PPP6C \\
\hline DUSP1 & MAPK8 \\
\hline KLK2 & SERPINA3 \\
\hline MAP3K5 & PPP5C \\
\hline RPLP1 & RPLP1 \\
\hline PLAT & SERPINI1 \\
\hline ARL4D & TLE1 \\
\hline PAK1 & RAC1 \\
\hline LMO4 & PITPNB \\
\hline NFKBIB & RELA \\
\hline EEF1A1 & EIF3F \\
\hline ELK1 & SRF \\
\hline CD3E & SYK \\
\hline FLNA & ITGB1 \\
\hline ATP5C1 & MPP1 \\
\hline TP53 & ZNF24 \\
\hline MLL & RBBP5 \\
\hline MAP2K3 & MAPK3 \\
\hline EGR1 & NAB1 \\
\hline MAP2K4 & МАР3К8 \\
\hline CASP8 & TRAF1 \\
\hline ESR1 & PTPN1 \\
\hline CNTF & IL6ST \\
\hline IRS1 & PTPN1 \\
\hline F2 & SERPINE1 \\
\hline RXRA & SMAD2 \\
\hline PIK3R1 & SRC \\
\hline МАР3К14 & RIPK1 \\
\hline RB1 & SNW1 \\
\hline CD8A & CD8B \\
\hline CHGB & PTEN \\
\hline CAPNS1 & GNB2 \\
\hline POLR2F & POLR2G \\
\hline BAK1 & HSPD1 \\
\hline CRKL & WAS \\
\hline CAV2 & NCK1 \\
\hline AR & PIAS1 \\
\hline BRAF & BRAF \\
\hline LCP2 & PLCG1 \\
\hline LMO1 & TAL1 \\
\hline SF1 & WWP2 \\
\hline $\mathrm{C7}$ & PLG \\
\hline CSF1R & FYN \\
\hline EIF4E & PML \\
\hline EGR1 & PITX1 \\
\hline
\end{tabular}




\begin{tabular}{|c|c|}
\hline КАТ2В & RARA \\
\hline VIPR1 & WASL \\
\hline MYOG & POLR2C \\
\hline C19orf57 & C19orf57 \\
\hline CHGB & $\mathrm{TAZ}$ \\
\hline BRCA1 & UBE2L3 \\
\hline RPA2 & XPA \\
\hline HDAC1 & TGIF1 \\
\hline DLG3 & GRIN2C \\
\hline FGFR1 & KPNB1 \\
\hline CBLB & GRB2 \\
\hline $\mathrm{ERH}$ & SETDB1 \\
\hline PKD2 & PKD2 \\
\hline PLSCR1 & VASP \\
\hline CCDC85B & ZNF638 \\
\hline SHB & ZAP70 \\
\hline CLINT1 & CLTC \\
\hline PLK4 & SFN \\
\hline FGR & WAS \\
\hline GNA13 & PRKCE \\
\hline FHL2 & FHL3 \\
\hline FKBP1A & ITPR1 \\
\hline NUP98 & NXF1 \\
\hline PSMC1 & PSMD2 \\
\hline FLNC & INPPL1 \\
\hline CRYAB & HSPB1 \\
\hline CDC6 & ORC3L \\
\hline CDK7 & MCM7 \\
\hline RFC3 & RFC4 \\
\hline HTT & SUMO1 \\
\hline GFAP & VIM \\
\hline NGFR & TRAF4 \\
\hline CBX5 & TRIM28 \\
\hline MSN & MSN \\
\hline RARA & RXRA \\
\hline FYN & PTPN5 \\
\hline PRMT1 & YLPM1 \\
\hline PSMD2 & UBE3C \\
\hline CSNK1D & DVL1 \\
\hline EGFR & GAB1 \\
\hline CEBPZ & TP73 \\
\hline HDAC1 & RBL2 \\
\hline LGALS1 & SPN \\
\hline FXR2 & SNAP23 \\
\hline HDAC2 & SP3 \\
\hline RPS19 & RPS19 \\
\hline PARP1 & RPS3A \\
\hline
\end{tabular}




\begin{tabular}{|c|c|}
\hline CASP2 & CASP2 \\
\hline TEC & WAS \\
\hline LPA & LRP2 \\
\hline FOS & NCOA1 \\
\hline CNTN1 & PTPRB \\
\hline PTN & TXNDC9 \\
\hline ITGB1 & YWHAB \\
\hline BRCA1 & MED1 \\
\hline CUL3 & SUMO2 \\
\hline IFNB1 & IFNB1 \\
\hline SP1 & SREBF2 \\
\hline ATN1 & LRP2 \\
\hline CDK6 & CDKN2A \\
\hline NDN & TP53 \\
\hline SMAD3 & TMED1 \\
\hline ETS1 & RBBP6 \\
\hline CREBBP & IRF3 \\
\hline PAPPA & PRG2 \\
\hline EP300 & SS18 \\
\hline GHR & GRB2 \\
\hline UCP3 & YWHAB \\
\hline SET & TAF1A \\
\hline HFE & TFRC \\
\hline CD28 & CD80 \\
\hline HLA & MAGEA1 \\
\hline ACVR1B & ACVR2B \\
\hline FBLN1 & FBLN1 \\
\hline $\mathrm{CDH} 2$ & PTPN1 \\
\hline TRIM26 & TRIM26 \\
\hline ID2 & TCF3 \\
\hline RABEP1 & RABEP1 \\
\hline IGFBP3 & POLA2 \\
\hline F10 & HIST1H1C \\
\hline HNRNPK & KHDRBS1 \\
\hline FGFR3 & GTF3C1 \\
\hline CSNK2A1 & SFN \\
\hline CRKL & PIK3R1 \\
\hline MAPK8IP2 & MAPK8IP2 \\
\hline CD2 & LCK \\
\hline NFKB2 & REL \\
\hline PRKCZ & RAF1 \\
\hline PAFAH1B3 & XRCC6 \\
\hline PRNP & STIP1 \\
\hline BMI1 & PTN \\
\hline CCNA2 & TP53 \\
\hline CSF3R & JAK1 \\
\hline S100A1 & S100A4 \\
\hline
\end{tabular}




\begin{tabular}{|c|c|}
\hline STAT1 & TYK2 \\
\hline GTF2F2 & JUN \\
\hline GSN & VCL \\
\hline MAML1 & NOTCH4 \\
\hline RRAD & TPM2 \\
\hline IL1R1 & PIK3R1 \\
\hline ITPR1 & PRKG1 \\
\hline HSPA1A & NR3C1 \\
\hline CBL & PIK3R2 \\
\hline CENPC1 & DAXX \\
\hline FN1 & ITGA5 \\
\hline GAPDH & LAMA4 \\
\hline HDAC1 & RAD9A \\
\hline CD86 & CTLA4 \\
\hline SUPT4H1 & SUPT5H \\
\hline WIPF2 & wwox \\
\hline CBLB & PIK3R1 \\
\hline EIF4G1 & PABPC1 \\
\hline FN1 & REG3A \\
\hline MAP2K5 & МАРЗКЗ \\
\hline CCL11 & DPP4 \\
\hline FHL2 & TRAF6 \\
\hline RRM1 & RRM1 \\
\hline PAH & PAH \\
\hline ABL1 & XRCC6 \\
\hline EEF1A1 & TP53BP2 \\
\hline DYNLL1 & PAK1 \\
\hline FLT4 & KDR \\
\hline BTG2 & PRMT1 \\
\hline CDK6 & CDKN2D \\
\hline PLK1 & PSMA5 \\
\hline C3 & CFHR4 \\
\hline AP2B1 & MLH1 \\
\hline$A R$ & NONO \\
\hline MITF & SPI1 \\
\hline CBL & CSF1R \\
\hline C1R & SERPING1 \\
\hline AMPH & VTN \\
\hline PHYHIP & TTR \\
\hline RBL1 & SNW1 \\
\hline PPARD & SRC \\
\hline DNTT & DNTTIP2 \\
\hline EMD & LMNA \\
\hline LRP1 & THBS1 \\
\hline GATA2 & JUN \\
\hline NFKB2 & NFKBIA \\
\hline PAPPA & SMAD2 \\
\hline
\end{tabular}




\begin{tabular}{|c|c|}
\hline PSME2 & PSME2 \\
\hline GTF2E1 & GTF2E2 \\
\hline F3 & PLG \\
\hline FAS & LCK \\
\hline DAB2 & SMAD2 \\
\hline TNFRSF25 & TRADD \\
\hline CBL & SRC \\
\hline DLG4 & PRKCA \\
\hline RPL28 & SMAD4 \\
\hline GTF2B & GTF2E2 \\
\hline FBLN2 & NID1 \\
\hline CDH3 & $\mathrm{CDH} 3$ \\
\hline LTBR & TRAF4 \\
\hline ADD1 & SPTA1 \\
\hline GLI3 & SKI \\
\hline BCL2L1 & CASP9 \\
\hline МАР3К12 & TSC22D1 \\
\hline MDM2 & TAF1 \\
\hline CASP4 & IL18 \\
\hline EWSR1 & HMGA1 \\
\hline RXRA & THRB \\
\hline MAD1L1 & MAX \\
\hline PTPRJ & SDCBP \\
\hline HDAC2 & PTMA \\
\hline CAPN1 & NFKBIA \\
\hline CNTF & IL6R \\
\hline GRIK2 & SDCBP \\
\hline KRT18 & PRKCE \\
\hline CDKN2B & MAGEA11 \\
\hline PTH & PTH2R \\
\hline ATM & TP53 \\
\hline IK & PFN2 \\
\hline TAF11 & TAF12 \\
\hline COMMD1 & TCEB1 \\
\hline CSNK2A2 & CSNK2B \\
\hline DARS & EEF1A1 \\
\hline CCNA2 & CDKN1A \\
\hline DRD2 & KCNJ6 \\
\hline BRF1 & TBP \\
\hline CPSF6 & WWP1 \\
\hline ATP1A1 & $\mathrm{EZH} 2$ \\
\hline $\mathrm{HTT}$ & SP1 \\
\hline CRYBA4 & CRYBB2 \\
\hline EIF4B & EIF4B \\
\hline SPTA1 & VASP \\
\hline SUMO1 & TOP2B \\
\hline ARC & KRT15 \\
\hline
\end{tabular}




\begin{tabular}{|c|c|}
\hline CD28 & LRRC23 \\
\hline CSNK2A1 & KLF1 \\
\hline PSME3 & PSME3 \\
\hline EPHB2 & ITSN1 \\
\hline TFPI & THBS1 \\
\hline RAD52 & UBE2I \\
\hline MECP2 & SMARCA2 \\
\hline TAF1 & TAF9 \\
\hline PRM1 & SRPK1 \\
\hline CHGB & POLD1 \\
\hline RUNX2 & XRCC5 \\
\hline CLK2 & CLK3 \\
\hline $\mathrm{NCL}$ & S100A11 \\
\hline IL1A & S100A13 \\
\hline CSTA & PTN \\
\hline PSMD11 & TP53 \\
\hline SKP1 & SKP2 \\
\hline CDC37 & CDK4 \\
\hline HNRNPD & PABPC1 \\
\hline SNAP23 & VAMP7 \\
\hline NCF2 & NCF4 \\
\hline ATF1 & CSNK2A1 \\
\hline BARD1 & MSH2 \\
\hline TAC1 & TACR3 \\
\hline CDK2 & RBL1 \\
\hline SRC & SYK \\
\hline ATM & BRCA1 \\
\hline ENO2 & ST3GAL2 \\
\hline MY07A & PRKAR1A \\
\hline C16orf45 & ENO2 \\
\hline NME1 & RORA \\
\hline ACCN2 & PRKACA \\
\hline PSMD2 & ZBTB16 \\
\hline TOP1 & TP53 \\
\hline DNM1 & SH3GL1 \\
\hline PECAM1 & XRCC6 \\
\hline DLG1 & GRIN2B \\
\hline GNB2 & RAF1 \\
\hline LIFR & OSM \\
\hline JUP & PECAM1 \\
\hline F10 & F8 \\
\hline CASP9 & NAIP \\
\hline MCM5 & SSRP1 \\
\hline GNB2L1 & RASA1 \\
\hline PGF & PGF \\
\hline INPP5D & LYN \\
\hline RUNX3 & SMAD3 \\
\hline
\end{tabular}




\begin{tabular}{|c|c|}
\hline ATP2B4 & DLG1 \\
\hline CD27 & TRAF3 \\
\hline PIK3R1 & SSTR2 \\
\hline TRADD & TRAF1 \\
\hline ANXA2 & $\mathrm{APOH}$ \\
\hline SF3A1 & SF3A3 \\
\hline ARHGAP1 & PIK3R1 \\
\hline SMAD3 & SREBF2 \\
\hline GRB10 & KIT \\
\hline TLK1 & TLK2 \\
\hline PRKCA & RGS2 \\
\hline CANX & LCT \\
\hline UCP3 & YWHAZ \\
\hline HK2 & PRKCE \\
\hline HNRNPK & RBMX \\
\hline ATP6AP1 & HBA1 \\
\hline ACP5 & ZBTB16 \\
\hline STX1A & TXLNA \\
\hline PDX1 & PDX1 \\
\hline STK38 & YWHAZ \\
\hline ERBB4 & YAP1 \\
\hline NONO & NONO \\
\hline EYA1 & SIX1 \\
\hline PPP1R8 & PRKACA \\
\hline SMAD1 & XPC \\
\hline CAP1 & CAP1 \\
\hline GSTP1 & PNO1 \\
\hline KRAS & RASSF2 \\
\hline CTBP1 & PNN \\
\hline PIK3R2 & YWHAB \\
\hline ADA & ADORA1 \\
\hline HIF1A & HSP90AA1 \\
\hline CEBPA & RUNX1 \\
\hline BMPR2 & GDF5 \\
\hline PABPC4 & YWHAZ \\
\hline TNFRSF1A & TRAP1 \\
\hline OGT & TRAK1 \\
\hline SKIL & SKIL \\
\hline DDB2 & XPC \\
\hline PTPN12 & WAS \\
\hline RPA3 & RPA3 \\
\hline RIPK1 & TNFRSF1A \\
\hline SNRNP70 & SNRPC \\
\hline MYCBP & PTN \\
\hline YWHAZ & YWHAZ \\
\hline CREBBP & MAF \\
\hline ADAM17 & MAD2L1 \\
\hline
\end{tabular}




\begin{tabular}{|c|c|}
\hline CXCL12 & DPP4 \\
\hline LTA & UMOD \\
\hline FGF1 & FGFR4 \\
\hline PSMD11 & SMAD3 \\
\hline BAT3 & EDN1 \\
\hline PLK3 & TRIP13 \\
\hline ITGA8 & ITGB1 \\
\hline PFN2 & RPS10 \\
\hline MAPK14 & ZFP36L1 \\
\hline FGF12 & MAPK8IP2 \\
\hline RAF1 & SRC \\
\hline DAZAP2 & LMO2 \\
\hline DST & ITGB4 \\
\hline PTEN & SLC9A3R2 \\
\hline FCGR2A & LYN \\
\hline CTNNA1 & CTNNB1 \\
\hline AKT1 & SMAD7 \\
\hline DLG1 & KCNJ4 \\
\hline SPTA1 & SPTB \\
\hline CD36 & LYN \\
\hline IKZF1 & RBBP8 \\
\hline PTPN12 & PXN \\
\hline NUP214 & NXF1 \\
\hline GRB2 & SELL \\
\hline CALR & PLAT \\
\hline CIB1 & PSEN2 \\
\hline TOP2A & YWHAE \\
\hline KHDRBS1 & SMARCA2 \\
\hline DDB1 & DDB2 \\
\hline CDC25C & MAPK14 \\
\hline DLST & FLII \\
\hline RELB & USP11 \\
\hline ABL1 & PTPN6 \\
\hline HLA & KIR2DL3 \\
\hline MSX2 & MSX2 \\
\hline GNAQ & PIK3CA \\
\hline DNTTIP2 & ESR2 \\
\hline CDC6 & CDK2 \\
\hline MEF2A & SUMO1 \\
\hline PPP1R8 & SETDB1 \\
\hline DLG2 & KCNJ4 \\
\hline CRHR1 & GNAQ \\
\hline GRB10 & RAF1 \\
\hline MRE11A & XRCC6 \\
\hline AP3D1 & AP3S2 \\
\hline ARHGEF2 & YWHAZ \\
\hline NDRG1 & S100B \\
\hline
\end{tabular}




\begin{tabular}{|c|c|}
\hline KPNB1 & RANBP2 \\
\hline DAXX & PAX5 \\
\hline BAG1 & RARA \\
\hline CCDC85B & PSMA1 \\
\hline TAF13 & TAF15 \\
\hline KRT15 & SMARCD1 \\
\hline APOB & CALR \\
\hline CSK & SHC1 \\
\hline SKIL & SPARCL1 \\
\hline CDK7 & MNAT1 \\
\hline CREBBP & TCF12 \\
\hline CD58 & DNAJA1 \\
\hline RPLPO & SMAD3 \\
\hline NFKBIA & PTPN1 \\
\hline ADRB2 & EIF2B1 \\
\hline KRT15 & KRT81 \\
\hline NCOA1 & $\mathrm{NR} 1 \mathrm{H} 4$ \\
\hline BMPR1B & BMPR2 \\
\hline RAD51 & RAD51 \\
\hline MPP3 & SCN2A \\
\hline TNNC1 & TNNT1 \\
\hline TP53 & TSG101 \\
\hline CD3E & NCK1 \\
\hline COPS6 & PDZK1IP1 \\
\hline E2F1 & SP2 \\
\hline BMPR2 & CRYAB \\
\hline CASP6 & CASP8 \\
\hline FXR2 & KRT20 \\
\hline VASP & $\mathrm{VCL}$ \\
\hline APPBP2 & NFYA \\
\hline BCL2L2 & BIK \\
\hline PDK1 & PKN2 \\
\hline $\mathrm{BCL} 2$ & MAPK1 \\
\hline CRYBB2 & CRYBB2 \\
\hline GLI1 & ZIC1 \\
\hline PIP4K2A & RAC1 \\
\hline CBLB & EGFR \\
\hline COPS2 & COPS8 \\
\hline IL1A & IL1A \\
\hline LIMK1 & LIMK1 \\
\hline GHR & PTPRB \\
\hline PDGFRB & PTPN1 \\
\hline ALDH2 & HSPE1 \\
\hline EEF1A1 & RNF10 \\
\hline$A B \mid 2$ & KRT15 \\
\hline LGALS2 & LTA \\
\hline RGS6 & STMN2 \\
\hline
\end{tabular}




\begin{tabular}{|c|c|}
\hline JAK1 & TYK2 \\
\hline CAV2 & GOLGB1 \\
\hline CCDC85B & ZFP36 \\
\hline ADA & ADORA2A \\
\hline LCP2 & ZAP70 \\
\hline ACAN & FBLN2 \\
\hline E2F1 & NFKB1 \\
\hline NTRK3 & PLCG1 \\
\hline AP2B1 & TGFBR2 \\
\hline CCND1 & CDK4 \\
\hline CSNK2A1 & HSP90B1 \\
\hline CD9 & ITGB1 \\
\hline HSPA1A & YWHAZ \\
\hline PIAS1 & STAT1 \\
\hline GSTM1 & MAP3K5 \\
\hline CDKN1A & TK1 \\
\hline ALDOA & ATP6V1E1 \\
\hline TGFB1 & THBS1 \\
\hline ATP5C1 & EGFR \\
\hline CREBBP & MAML1 \\
\hline SNRPE & SNRPG \\
\hline LDLR & PF4 \\
\hline ATP5C1 & ITIH2 \\
\hline FYN & MAG \\
\hline BRCA1 & CCNB1 \\
\hline DOK1 & LYN \\
\hline CSTF2 & KAT5 \\
\hline EPHB3 & RASA1 \\
\hline APP & CTSD \\
\hline ARHGAP1 & ARHGAP1 \\
\hline EIF4A3 & YWHAQ \\
\hline$A R$ & XRCC5 \\
\hline FHIT & UBE2I \\
\hline IRF2 & RELA \\
\hline PABPC1 & PXN \\
\hline PARP1 & RELA \\
\hline UBE2I & UCHL1 \\
\hline ITGB1 & PRKCA \\
\hline PSMD11 & SMAD2 \\
\hline TNFRSF4 & TRAF5 \\
\hline HRAS & PIK3CG \\
\hline GRB2 & HNRNPC \\
\hline AHR & ARNT \\
\hline HDAC1 & TAL1 \\
\hline LTBR & TRAF5 \\
\hline RBPJ & SNW1 \\
\hline ATR & RHEB \\
\hline
\end{tabular}




\begin{tabular}{|c|c|}
\hline GNAI3 & GPSM2 \\
\hline LASP1 & ZYX \\
\hline E2F4 & TFDP2 \\
\hline CHD8 & CTNNB1 \\
\hline COPS6 & SAT1 \\
\hline BAT3 & CDK4 \\
\hline APEX1 & TP53 \\
\hline CD63 & CD9 \\
\hline THRB & TP53 \\
\hline CDKN1A & ESR1 \\
\hline CR2 & IFNA1 \\
\hline DHX9 & KHDRBS1 \\
\hline MEOX2 & PAX3 \\
\hline CITED2 & EP300 \\
\hline PTPN11 & SLAMF1 \\
\hline NFKB1 & TSC22D3 \\
\hline TGFB2 & TGFBR2 \\
\hline NTRK2 & $\mathrm{SHC} 3$ \\
\hline AP3S1 & IRS1 \\
\hline TOPBP1 & ZBTB17 \\
\hline ATN1 & BAT3 \\
\hline FGF2 & VTN \\
\hline CAPZA1 & S100B \\
\hline ITPR3 & TRPC1 \\
\hline SPARC & VEGFA \\
\hline CALCOCO2 & SHC1 \\
\hline AP2M1 & LY9 \\
\hline APP & HSD17B10 \\
\hline DAXX & SLC2A4 \\
\hline CFL1 & MYCBP \\
\hline APC & SFN \\
\hline EPHB2 & RYK \\
\hline C1QBP & PRKD1 \\
\hline MPRIP & RHOA \\
\hline LAPTM5 & WWP1 \\
\hline ESR1 & SRC \\
\hline AKT1 & HSP90AA1 \\
\hline RPGR & SMC1A \\
\hline TRIM22 & USP7 \\
\hline NFIB & NFIX \\
\hline NROB2 & RARA \\
\hline SUMO1 & TOP1 \\
\hline BIRC2 & GSPT1 \\
\hline CCNA1 & MYBL2 \\
\hline AKT1 & TOPBP1 \\
\hline MTIF2 & NFKBIB \\
\hline RPA2 & RPA3 \\
\hline
\end{tabular}




\begin{tabular}{|c|c|}
\hline AR & HDAC1 \\
\hline HSP90AA1 & PTGES3 \\
\hline HOXA9 & PBX1 \\
\hline MDM2 & MDM2 \\
\hline TJP2 & YWHAZ \\
\hline PTS & PTS \\
\hline ESR1 & ISL1 \\
\hline IGFALS & IGFBP3 \\
\hline FGFBP1 & FGFBP1 \\
\hline CGA & PTN \\
\hline CCNG1 & TP53 \\
\hline PPP2R1A & PPP2R2A \\
\hline MCF2 & MCF2 \\
\hline BMP2 & COL2A1 \\
\hline AFAP1 & PRKCG \\
\hline STX4 & TXLNA \\
\hline CDK9 & SUPT5H \\
\hline POLR2C & POLR2K \\
\hline CREBBP & TDG \\
\hline $\mathrm{CDH} 6$ & $\mathrm{CDH} 6$ \\
\hline CUL4B & DDB1 \\
\hline ARPC2 & ARPC4 \\
\hline PDHA2 & PDHB \\
\hline FOS & XBP1 \\
\hline $\mathrm{NCL}$ & TP53 \\
\hline ENO2 & MAP4 \\
\hline BCL3 & FOS \\
\hline MT2A & PRKD1 \\
\hline F10 & TFPI \\
\hline JUN & SP1 \\
\hline GHR & PTPN9 \\
\hline HSP90AA1 & SRC \\
\hline EWSR1 & SNRPC \\
\hline SMAD4 & TGFBR1 \\
\hline EEF1G & SKIL \\
\hline CASP2 & CRADD \\
\hline MUTYH & PCNA \\
\hline CSNK1E & OCLN \\
\hline CTNNB1 & USP9X \\
\hline EWSR1 & SLC1A1 \\
\hline ATP1A3 & ATXN1 \\
\hline CFDP1 & EWSR1 \\
\hline ABL1 & WASF1 \\
\hline ITK & KHDRBS1 \\
\hline MYL2 & PAK2 \\
\hline HIC1 & HIC1 \\
\hline SMN1 & SNRPE \\
\hline
\end{tabular}




\begin{tabular}{|c|c|}
\hline HMGA2 & NFKB1 \\
\hline FEZ1 & PTH \\
\hline TNNT2 & TPM1 \\
\hline SPTAN1 & SPTB \\
\hline GTF2I & MYC \\
\hline MED1 & VDR \\
\hline FAS & UBE2I \\
\hline KRT5 & PKP2 \\
\hline GRB10 & SRC \\
\hline CDC25B & YWHAB \\
\hline MLL & MLL \\
\hline RHOA & TRPC1 \\
\hline ID1 & MYF6 \\
\hline RAB9A & RABEPK \\
\hline CRKL & GAB1 \\
\hline DAB2 & TGFBR1 \\
\hline PCBP1 & PDLIM7 \\
\hline ESR2 & SP1 \\
\hline CD2 & LGALS1 \\
\hline HSD17B1 & HSD17B1 \\
\hline MITF & UBE2I \\
\hline POLR2C & POLR2L \\
\hline CDC27 & PIN1 \\
\hline SUMO2 & TDG \\
\hline RAF1 & YWHAH \\
\hline COPA & SLC25A11 \\
\hline INPP5D & $\mathrm{KIT}$ \\
\hline HIF1A & JUN \\
\hline SKIL & SMAD4 \\
\hline KAT5 & SAT1 \\
\hline KRT18 & PKD1 \\
\hline ILK & ITGB3 \\
\hline TAF1B & TBP \\
\hline NROB2 & NR1H3 \\
\hline AKAP13 & YWHAZ \\
\hline CANX & ITGB1 \\
\hline ALDH2 & CRMP1 \\
\hline CD247 & UNC119 \\
\hline CD79B & SYK \\
\hline$A R$ & DAP3 \\
\hline GNRH1 & MEP1A \\
\hline PARP1 & XRCC6 \\
\hline APBA2 & RELA \\
\hline TAF15 & TAF5 \\
\hline MCM2 & MPP3 \\
\hline COL1A1 & PRELP \\
\hline DVL1 & SMAD4 \\
\hline
\end{tabular}




\begin{tabular}{|c|c|}
\hline $\mathrm{CCNH}$ & ESR1 \\
\hline PCDH1 & SMAD3 \\
\hline ARR3 & ARR3 \\
\hline PCNA & XRCC6 \\
\hline FTH1 & FXR2 \\
\hline TAF1 & TAF5 \\
\hline DNTTIP2 & RXRA \\
\hline HMOX1 & HMOX1 \\
\hline TAF12 & TBP \\
\hline NR3C1 & SMAD3 \\
\hline RAF1 & TSC22D3 \\
\hline FLT4 & SHC1 \\
\hline NEDD8 & UCHL1 \\
\hline FLI1 & GATA1 \\
\hline CHGB & S100A8 \\
\hline LIPE & STAR \\
\hline MAP2K1 & MAP2K1 \\
\hline XRCC6 & YWHAZ \\
\hline CASP8 & NR1H4 \\
\hline KAT5 & PLA2G4A \\
\hline AKT1 & TERT \\
\hline ATXN1 & NR4A1 \\
\hline F13A1 & F13B \\
\hline $\mathrm{CDH} 1$ & IQGAP1 \\
\hline CYP11A1 & SMAD3 \\
\hline CCNE1 & RBL2 \\
\hline CRKL & STAT5A \\
\hline CFL1 & LIMK1 \\
\hline NCOR2 & PPARG \\
\hline VHL & ZNF197 \\
\hline DOK1 & HCK \\
\hline CCNA2 & RBL1 \\
\hline F10 & PLG \\
\hline HNF4A & NCOA6 \\
\hline RAD23A & SQSTM1 \\
\hline ANXA11 & PLSCR1 \\
\hline PLA2G1B & PLA2G2A \\
\hline POLA1 & POLA2 \\
\hline GGA3 & RABEP1 \\
\hline LIG1 & MRE11A \\
\hline CENPF & TOP3В \\
\hline PSMD11 & SETDB1 \\
\hline ERBB2 & HSP90B1 \\
\hline GRB2 & PTPN12 \\
\hline UNC119 & ZNF24 \\
\hline EIF3C & EIF3E \\
\hline MSX2 & XRCC5 \\
\hline
\end{tabular}




\begin{tabular}{|c|c|}
\hline HTT & MAP3К10 \\
\hline EBI3 & MDFI \\
\hline CAV1 & CAV2 \\
\hline BRCA1 & СТВP1 \\
\hline HIST2H2BE & NAP1L4 \\
\hline CALR & NR3C1 \\
\hline NEDD9 & NEDD9 \\
\hline BMP2 & ENG \\
\hline МАРЗКЗ & MAP3K5 \\
\hline PTPN1 & SRC \\
\hline LDHB & LDHB \\
\hline C5 & C5AR1 \\
\hline CSTA & CTSL1 \\
\hline $\mathrm{CBL}$ & LYN \\
\hline CAV1 & GJA1 \\
\hline ARNTL & NPAS2 \\
\hline DGCR14 & LYST \\
\hline EXT1 & TRAP1 \\
\hline MYLK & SRC \\
\hline COPS6 & STX5 \\
\hline AXIN1 & EEF1A1 \\
\hline MCM7 & RBL2 \\
\hline ADD1 & CALM1 \\
\hline PARP1 & XRCC1 \\
\hline CLU & LRP2 \\
\hline EIF3A & YWHAZ \\
\hline ADRBK1 & GNAQ \\
\hline RB1 & TRIP11 \\
\hline KAT5 & PTPRS \\
\hline EP300 & ZBTB17 \\
\hline GNAI2 & PLSCR1 \\
\hline TCF3 & UBE2I \\
\hline KIT & MATK \\
\hline BIRC2 & CASP9 \\
\hline IGF1 & IGFBP4 \\
\hline BMI1 & RING1 \\
\hline E2F5 & TFDP1 \\
\hline NPPC & NPR2 \\
\hline EP300 & MAML1 \\
\hline GBP2 & SEPHS1 \\
\hline NFKB1 & NFKB1 \\
\hline ERBB2 & IL6ST \\
\hline CYTH2 & SFN \\
\hline FGFR3 & HNRNPL \\
\hline CBLB & NCK1 \\
\hline CBL & SLA \\
\hline ATXN1 & U2AF2 \\
\hline
\end{tabular}




\begin{tabular}{|c|c|}
\hline COPS6 & MNAT1 \\
\hline AKAP12 & PRKCA \\
\hline MEF2A & MYOG \\
\hline СТВP1 & КАT2B \\
\hline ATP6VOC & MARK3 \\
\hline GTF2E1 & GTF2F2 \\
\hline IL6 & SH3GL2 \\
\hline PIN1 & PLK1 \\
\hline IGF2 & VTN \\
\hline ACVR2A & INHBA \\
\hline HBA1 & HBB \\
\hline HLA & KIR2DL4 \\
\hline EEF1G & EEF1G \\
\hline FGB & LPA \\
\hline HNRNPU & NR3C1 \\
\hline ITGA4 & PXN \\
\hline COL4A2 & SKIL \\
\hline LYN & PAK2 \\
\hline CXCL9 & CXCR3 \\
\hline CDC25C & PIN1 \\
\hline TRAF4 & USP7 \\
\hline TGFBR1 & TRAF6 \\
\hline TIMM17A & TIMM44 \\
\hline SCAMP1 & UBE3A \\
\hline HSF1 & TBP \\
\hline CBX1 & RPL12 \\
\hline POLR2A & POLR2F \\
\hline SCNN1B & STX1A \\
\hline LYST & MED12 \\
\hline DLG1 & EZR \\
\hline CASP10 & TRAF1 \\
\hline PPP2R1B & PPP2R5C \\
\hline NME1 & RORB \\
\hline CD81 & RBBP6 \\
\hline EEF1A1 & NEU1 \\
\hline PTPN11 & PXN \\
\hline CDK6 & CDKN1A \\
\hline ICAM1 & ITGB2 \\
\hline F3 & F7 \\
\hline DYNLL1 & RGS2 \\
\hline NPY & $\mathrm{PMCH}$ \\
\hline TRAF3 & TRAF3 \\
\hline$A R$ & NROB1 \\
\hline FLNA & PSEN1 \\
\hline ACTN2 & GRIN2B \\
\hline TNFRSF1B & TRAIP \\
\hline AR & GSK3B \\
\hline
\end{tabular}




\begin{tabular}{|c|c|}
\hline CSK & RASA1 \\
\hline SETDB1 & TXNDC9 \\
\hline CD5 & CD6 \\
\hline CD40 & TRAF3 \\
\hline MAPT & YWHAZ \\
\hline EFNB2 & EPHA3 \\
\hline HDAC1 & PITX2 \\
\hline AKT1 & GSK3B \\
\hline CYTH1 & TRIM23 \\
\hline JUN & STAT1 \\
\hline FCGR2A & PIK3R1 \\
\hline PTPN6 & SIRPA \\
\hline STX1A & VAMP7 \\
\hline BLOC1S1 & PTN \\
\hline IRAK1 & MYD88 \\
\hline CSNK2A1 & JUN \\
\hline BMPR1A & GDF5 \\
\hline RUNX2 & YAP1 \\
\hline EGFR & EGFR \\
\hline SCAMP1 & TP53 \\
\hline EGFR & PLSCR1 \\
\hline $\mathrm{BCR}$ & TP53 \\
\hline AKT1 & BRAF \\
\hline TAF7 & TAF9 \\
\hline СТВР1 & TGIF1 \\
\hline EIF4G3 & PABPC1 \\
\hline CSNK1A1 & HMGB1 \\
\hline HSPA4 & МАРЗКЗ \\
\hline DLG4 & LRP2 \\
\hline RELA & TAF4B \\
\hline IRF1 & IRF8 \\
\hline PSMD4 & RAD23B \\
\hline CNTN1 & CNTN2 \\
\hline CHGB & UBTF \\
\hline EP300 & NPAS2 \\
\hline IGF2 & IGFBP5 \\
\hline FGFR2 & ITGA5 \\
\hline EIF2AK2 & TARBP2 \\
\hline TNFRSF17 & TRAF3 \\
\hline PIAS1 & SMAD7 \\
\hline NCOA1 & PGR \\
\hline PDIA3 & TAP1 \\
\hline SMAD1 & SMAD1 \\
\hline APP & BGN \\
\hline MLLT3 & PTN \\
\hline ARHGEF2 & RHOA \\
\hline CFLAR & MAP2K 4 \\
\hline
\end{tabular}




\begin{tabular}{|c|c|}
\hline MAP4K2 & RAB8A \\
\hline ANK3 & SMAD3 \\
\hline TNF & TNF \\
\hline HNRNPH1 & NCBP1 \\
\hline CAV1 & NOS3 \\
\hline NOS1 & SNTA1 \\
\hline WASL & WIPF1 \\
\hline ATN1 & EWSR1 \\
\hline CD3E & PIK3R1 \\
\hline TAF4 & TAF7 \\
\hline FN1 & $\mathrm{MIA}$ \\
\hline CFLAR & RIPK1 \\
\hline TEAD1 & VGLL1 \\
\hline MMP2 & TGFB1 \\
\hline SIAH1 & SIAH1 \\
\hline MAPK1 & SNCA \\
\hline PGR & UBE3A \\
\hline BCAP31 & $\mathrm{BCL} 2$ \\
\hline EIF3A & EIF4B \\
\hline PSMC3 & TRAF6 \\
\hline ADAM15 & NCK1 \\
\hline JUN & PIN1 \\
\hline CRKL & PDGFRA \\
\hline TTR & TTR \\
\hline CD7 & LGALS1 \\
\hline AR & CCND1 \\
\hline$A R$ & PTEN \\
\hline EEF1A1 & PLAUR \\
\hline ATN1 & RBPMS \\
\hline NR4A1 & VHL \\
\hline MDM2 & RPL23 \\
\hline ESR1 & PTGES3 \\
\hline $\mathrm{CCNH}$ & PSMA1 \\
\hline CD36 & FYN \\
\hline KPNA4 & RECQL \\
\hline EZH2 & ZMYND11 \\
\hline PIK3R2 & TGFBR1 \\
\hline ITPR1 & RHOA \\
\hline PML & SP1 \\
\hline BAT3 & FKBP2 \\
\hline SMN1 & SNRPD3 \\
\hline ECH1 & EEF1G \\
\hline C5 & $\mathrm{C7}$ \\
\hline CTSS & SERPINB3 \\
\hline FKBP3 & HDAC1 \\
\hline CSNK1A1 & ERF \\
\hline BAT1 & TUBB3 \\
\hline
\end{tabular}




\begin{tabular}{|c|c|}
\hline SMAD3 & SMAD3 \\
\hline $\mathrm{A} 2 \mathrm{M}$ & APOE \\
\hline HSPE1 & UNC119 \\
\hline KAT2A & XRCC6 \\
\hline COPS2 & NROB1 \\
\hline HNF1A & HNF1B \\
\hline LIMK1 & LIMK2 \\
\hline CITED2 & LHX2 \\
\hline FBLN2 & FBLN2 \\
\hline$A B C D 1$ & $\mathrm{ABCD} 3$ \\
\hline APC & MAPRE1 \\
\hline SNRPD3 & SNRPG \\
\hline PPIA & PRLR \\
\hline ATP5C1 & POLA2 \\
\hline BAD & RPS6KA1 \\
\hline CSF3R & SYK \\
\hline EIF2AK2 & STAT3 \\
\hline S100A1 & S100A1 \\
\hline PDLIM7 & TPM2 \\
\hline CSNK2A2 & $\mathrm{NCL}$ \\
\hline KAT2B & TWIST1 \\
\hline PTPRN & SNTB2 \\
\hline FUS & YBX1 \\
\hline MYC & PFDN5 \\
\hline $\mathrm{CCNH}$ & TP53 \\
\hline TBL3 & USP11 \\
\hline TAF1 & TAF7 \\
\hline NUP214 & XPO1 \\
\hline ADA & DRD1 \\
\hline ATM & MDC1 \\
\hline MYLK & PRKG2 \\
\hline SMAD7 & TGFBR1 \\
\hline GTF2I & USF1 \\
\hline CXCR3 & PF4 \\
\hline CUL1 & NEDD8 \\
\hline APC & DLGAP1 \\
\hline CEACAM1 & SHC1 \\
\hline TP53 & TP53 \\
\hline EED & EED \\
\hline ARHGAP5 & RND3 \\
\hline CSNK2A1 & MGMT \\
\hline DUSP1 & MAPK3 \\
\hline $\mathrm{ECH} 1$ & TIMP1 \\
\hline SFPQ & WWOX \\
\hline GRB2 & KRT8 \\
\hline PSMC5 & TNNT1 \\
\hline PTPN6 & VAV1 \\
\hline
\end{tabular}




$\begin{array}{ll}\text { CSRP3 } & \text { MYOD1 } \\ \text { ESR1 } & \text { KAT5 } \\ \text { APOE } & \text { MAPT } \\ \text { CSNK2A1 } & \text { DVL3 } \\ \text { PRKCSH } & \text { RGS2 } \\ \text { YWHAH } & \text { ZFP36 } \\ \text { DLG1 } & \text { KCNA3 } \\ \text { BRCA1 } & \text { RBBP8 } \\ \text { C19orf57 } & \text { EWSR1 } \\ \text { IL16 } & \text { KCN15 } \\ \text { CCND1 } & \text { THRA } \\ \text { IRS1 } & \text { PTPN11 } \\ \text { DLST } & \text { UMPS } \\ \text { FGF7 } & \text { FGFR2 } \\ \text { IK } & \text { NUP62 } \\ \text { PRLR } & \text { PTPN11 } \\ \text { ITGB1 } & \text { ITGB1BP1 } \\ \text { RARB } & \text { SMAD2 } \\ \text { FOXO1 } & \text { SMAD4 } \\ \text { CSNK2A2 } & \text { FOS } \\ \text { FYN } & \text { PXN } \\ \text { EWSR1 } & \text { MVK } \\ \text { SH3GL3 } & \text { TERF1 } \\ \text { RYR1 } & \text { TRDN } \\ \text { GNB2L1 } & \text { NSMAF } \\ \text { HCFC1 } & \text { OGT } \\ \text { RPLP1 } & \text { SAT1 } \\ \text { FLNA } & \text { VHL } \\ \text { ADAM17 } & \text { PTPN3 } \\ \text { KHDRBS1 } & \text { KHDRBS1 } \\ \text { APBA2 } & \text { LRP2 } \\ \text { RAC1 } & \text { WAS } \\ \text { HDAC1 } & \text { PCNA } \\ \text { LRP1 } & \text { MMP14 } \\ \text { TCF3 } & \text { TWIST1 } \\ \text { CREB3 } & \text { EMD } \\ \text { DLG4 } & \text { DYNLL1 } \\ \text { APP } & \text { SHC1 } \\ \text { CREB1 } & \text { RAB1A } \\ \text { MAP3K3 } & \text { ZBTB16 } \\ \text { APP } & \text { SNCA } \\ \text { ATF2 } & \text { NCOA6 } \\ \text { SMAD5 } & \text { WWP1 } \\ \text { JAK1 } & \text { PTPN6 } \\ \text { APP } & \text { APPBP2 } \\ \text { INSR } \\ \text { YWHAE }\end{array}$




\begin{tabular}{|c|c|}
\hline$A R$ & GTF2F2 \\
\hline RGS1 & TSC22D1 \\
\hline NCOA6 & NR3C1 \\
\hline DOCK2 & VAV1 \\
\hline ELN & FBLN1 \\
\hline HSPG2 & PRELP \\
\hline MASP1 & SERPING1 \\
\hline EIF4G1 & HSPB1 \\
\hline SETDB1 & USP11 \\
\hline GTF2B & JUN \\
\hline ELF4 & SKP2 \\
\hline COL4A3 & MFAP2 \\
\hline LEPR & SNX1 \\
\hline SMAD3 & XRCC6 \\
\hline STAT1 & TNFRSF1A \\
\hline ACTG1 & PFN2 \\
\hline CSNK2A1 & RELA \\
\hline CBX1 & CBX1 \\
\hline RAB5A & STX4 \\
\hline IL8 & SDC1 \\
\hline CD8A & HLA \\
\hline ERBB2 & SRC \\
\hline DNTT & XRCC6 \\
\hline PRKAR1A & PRKX \\
\hline IL13RA1 & TYK2 \\
\hline APBA1 & CACNA1B \\
\hline $\mathrm{BMX}$ & PTPN21 \\
\hline PLSCR1 & SLC35A2 \\
\hline EP300 & NFYB \\
\hline CRKL & INPP5D \\
\hline KHDRBS1 & PTK6 \\
\hline DRD3 & FLNA \\
\hline MAGEA11 & MLF1 \\
\hline IFNAR1 & TYK2 \\
\hline CTNNB1 & PSEN1 \\
\hline RBBP8 & RBL1 \\
\hline $\mathrm{CDC} 27$ & MAD2L1 \\
\hline RASA1 & YES1 \\
\hline BCL6 & JUNB \\
\hline PIK3R2 & TGFBR2 \\
\hline EFNA1 & PPBP \\
\hline CD63 & ITGB1 \\
\hline ANK2 & SIGMAR1 \\
\hline EPB42 & SPTAN1 \\
\hline CDC42EP1 & FHL3 \\
\hline ITGA2B & ITGB3 \\
\hline DLST & PFN2 \\
\hline
\end{tabular}




$\begin{array}{ll}\text { HIPK3 } & \text { SUMO1 } \\ \text { PTK2B } & \text { PTPN11 } \\ \text { BAK1 } & \text { BCL2L1 } \\ \text { LPL } & \text { LRP1 } \\ \text { CIRBP } & \text { PRMT1 } \\ \text { ANP32A } & \text { HSPB3 } \\ \text { FOS } & \text { MITF } \\ \text { LCK } & \text { MS4A1 } \\ \text { KAT5 } & \text { RGL2 } \\ \text { CRMP1 } & \text { RGL2 } \\ \text { DCTN2 } & \text { RPGR } \\ \text { ICAM2 } & \text { MSN } \\ \text { BMP7 } & \text { BMPR1B } \\ \text { MAGEA4 } & \text { UQCRB } \\ \text { APC } & \text { TFAP2A } \\ \text { ALK } & \text { PLCG1 } \\ \text { CD44 } & \text { EGFR } \\ \text { CCDC106 } & \text { KAT5 } \\ \text { TRIM23 } & \text { TRIM23 } \\ \text { BMP4 } & \text { BMPR1A } \\ \text { RIPK1 } & \text { SOSTM1 } \\ \text { TNFRSF8 } & \text { TRAF1 } \\ \text { CLU } & \text { XRCC6 } \\ \text { WRN } & \text { XRCC5 } \\ \text { AR } & \text { EGFR } \\ \text { MAD2L1BP } & \text { SETDB1 } \\ \text { TOP2A } & \text { TOP2B } \\ \text { MYC } & \text { TBP } \\ \text { IL2RA } & \text { IL2RA } \\ \text { EEF1A1 } & \text { RPA2 } \\ \text { RAB1A } & \text { SYT5 } \\ \text { EZR } & \text { FAS } \\ \text { CFTR } & \text { CFTR } \\ \text { PKP2 } & \text { SFN } \\ \text { ERCC3 } & \text { MNAT1 } \\ \text { SNAP25 } & \text { STX4 } \\ \text { ERH } & \text { TP53 } \\ \text { BAT3 } & \text { IGFBP5 } \\ \text { ORC1L } & \text { TERF2 } \\ \text { USF2 } & \text { USF2 } \\ \text { SCN5A } & \text { SNTB2 } \\ \text { DTNA } & \text { KCNJ12 } \\ \text { NRIP1 } & \text { RXRA } \\ \text { SAT1 } & \text { TP53 } \\ \text { EAPDH } & \text { PRKCI } \\ \text { CALR } \\ \text { EGN }\end{array}$




\begin{tabular}{|c|c|}
\hline AKT1 & FANCA \\
\hline FLNB & ITGB1 \\
\hline ATP2A1 & PLN \\
\hline TAF10 & TAF13 \\
\hline CSF3R & GRB2 \\
\hline PHB & SMARCA2 \\
\hline SUMO2 & TP53BP2 \\
\hline PLAGL1 & TP53 \\
\hline ELN & LGALS3 \\
\hline SNRPB & ТОР3В \\
\hline HHEX & PML \\
\hline FHL1 & FHL2 \\
\hline ADORA1 & GRM1 \\
\hline HSPA9 & TP53 \\
\hline GRB7 & SFN \\
\hline EEF1A1 & PFN2 \\
\hline RAC1 & SET \\
\hline ATF1 & CREBBP \\
\hline PRKCD & TIAM1 \\
\hline ESR1 & $\mathrm{NR} 2 \mathrm{C} 2$ \\
\hline $\mathrm{CDH} 5$ & FGA \\
\hline ATF3 & TP53 \\
\hline GRB14 & PRKCZ \\
\hline GOLGA2 & RAB1A \\
\hline SMAD4 & TM9SF2 \\
\hline HNRNPK & VAV1 \\
\hline DDR1 & PTPN11 \\
\hline AGTR1 & BDKRB2 \\
\hline ATXN1 & MBP \\
\hline $\mathrm{H} 2 \mathrm{AFX}$ & MDC1 \\
\hline SKIL & SMAD2 \\
\hline $\mathrm{APOH}$ & LRP8 \\
\hline FBLN2 & HSPG2 \\
\hline EYA2 & RBPMS \\
\hline ERBB3 & RGS4 \\
\hline CDK9 & MYBL2 \\
\hline JUN & RUNX2 \\
\hline MSH6 & PCNA \\
\hline CASP8 & VIM \\
\hline KRT8 & MAPK1 \\
\hline KHDRBS1 & PLCG1 \\
\hline AKT1 & BRCA1 \\
\hline NCOA1 & NFKB1 \\
\hline $\mathrm{BCHE}$ & $\mathrm{BCHE}$ \\
\hline MAP3К10 & MAPK8IP2 \\
\hline LTB & TNFRSF1A \\
\hline IL10 & IL10RA \\
\hline
\end{tabular}




\begin{tabular}{|c|c|}
\hline HNF4A & TP53 \\
\hline ACVR1B & SMAD7 \\
\hline NOTCH3 & SNW1 \\
\hline$B C R$ & GRB2 \\
\hline AXIN1 & SMAD7 \\
\hline SRC & SYN1 \\
\hline ADD1 & RFX1 \\
\hline GRB2 & MAP2 \\
\hline HSP90AA1 & PPARA \\
\hline HLA & TAP2 \\
\hline ACTA1 & $\mathrm{GC}$ \\
\hline NOLC1 & YWHAZ \\
\hline UCP2 & YWHAQ \\
\hline SMAD1 & UBA52 \\
\hline HTT & SH3GL3 \\
\hline FTL & KNG1 \\
\hline HSPA5 & TG \\
\hline RHEB & $\mathrm{TSC} 2$ \\
\hline GRAP & KIT \\
\hline GP1BB & YWHAZ \\
\hline GNAI1 & GPR143 \\
\hline ARF1 & CHRM3 \\
\hline TFDP1 & TP53 \\
\hline IRF3 & PRDX1 \\
\hline IRF4 & SPI1 \\
\hline HNRNPC & KRAS \\
\hline AKT1 & KRT10 \\
\hline DHPS & RPL9 \\
\hline CD46 & MSN \\
\hline CDC7 & MCM4 \\
\hline CKS2 & UNC119 \\
\hline АСТВ & VSNL1 \\
\hline СТВP1 & HIC1 \\
\hline MDM2 & PML \\
\hline CSF2RB & LYN \\
\hline NFKBIB & PDCD2 \\
\hline APEX1 & SET \\
\hline EP300 & NR4A1 \\
\hline TNFRSF14 & TRAF1 \\
\hline MDFI & SIX1 \\
\hline $\mathrm{ABI} 2$ & TRIM32 \\
\hline MAD2L1BP & TRIP13 \\
\hline LRP2 & PLAU \\
\hline ESR1 & ESR1 \\
\hline TDG & VDR \\
\hline PLXNB1 & SEMA4D \\
\hline NEDD4 & NEDD4 \\
\hline
\end{tabular}




\begin{tabular}{|c|c|}
\hline COPS5 & GFER \\
\hline GLG1 & SELE \\
\hline HSPH1 & UBA1 \\
\hline $\mathrm{BGN}$ & ELN \\
\hline CCNA1 & MCM6 \\
\hline $\mathrm{CDH} 1$ & JUP \\
\hline PTN & PTN \\
\hline CDK2 & RB1 \\
\hline GRB10 & MAP2K1 \\
\hline IMMT & RER1 \\
\hline HIPK3 & $\mathrm{NR} 2 \mathrm{~F} 2$ \\
\hline ACTA1 & PRKCE \\
\hline TSC2 & YWHAH \\
\hline S100A10 & TRPV6 \\
\hline PTMA & SET \\
\hline GNAQ & TBXA2R \\
\hline СCT5 & TCP1 \\
\hline PRKCD & SHC1 \\
\hline GTF2E1 & SND1 \\
\hline $\mathrm{BCL} 2$ & RAF1 \\
\hline ATRX & $\mathrm{EZH} 2$ \\
\hline NCK1 & PKN2 \\
\hline BGN & TGFB1 \\
\hline MVP & PARP4 \\
\hline FGFR3 & KRT8 \\
\hline NCOA1 & PPARA \\
\hline EP300 & MAF \\
\hline PRKCE & RAF1 \\
\hline CD3D & CD8A \\
\hline ARF3 & ARFIP1 \\
\hline ATXN1 & R3HDM1 \\
\hline CSK & PECAM1 \\
\hline RIPK1 & TRIM38 \\
\hline HSP90AA1 & PPP5C \\
\hline STK38 & YWHAB \\
\hline BRCA1 & JUN \\
\hline CCNA2 & ITGB3BP \\
\hline LRP1 & LRP1 \\
\hline COL18A1 & KDR \\
\hline ACTN1 & PYGB \\
\hline FUS & SF1 \\
\hline EPB41 & SPTB \\
\hline NFKB2 & NR3C1 \\
\hline SRC & SRC \\
\hline KLK3 & SERPINA5 \\
\hline AKAP10 & PRKAR1A \\
\hline RHO & SAG \\
\hline
\end{tabular}




\begin{tabular}{|c|c|}
\hline MYC & TIAM1 \\
\hline CDC20 & CDC20 \\
\hline EP300 & PTMA \\
\hline GBP2 & SERPINB9 \\
\hline GDI2 & RAB8A \\
\hline $\mathrm{CDH} 2$ & CTNNA1 \\
\hline ATF2 & MAPK8 \\
\hline ETS1 & MAF \\
\hline PPARG & RXRA \\
\hline GJA1 & PRKCE \\
\hline BAT3 & DPT \\
\hline EPRS & RARS \\
\hline ADA & NR3C1 \\
\hline HSP90AA1 & STAT3 \\
\hline TAX1BP1 & TNFAIP3 \\
\hline MEN1 & SMAD3 \\
\hline HSP90AA1 & TP53 \\
\hline FKBP1A & GLMN \\
\hline ESR2 & NRIP1 \\
\hline EFNB3 & EPHA4 \\
\hline GTF2I & STAT3 \\
\hline OCRL & RAB8A \\
\hline CAV1 & PTPN11 \\
\hline ARL3 & TLE1 \\
\hline TAP1 & TAP2 \\
\hline TP53 & XRCC6 \\
\hline EP300 & MEF2C \\
\hline CTNNB1 & NR5A1 \\
\hline KRT18 & TNFRSF1B \\
\hline NEFL & SPTAN1 \\
\hline ICAM3 & ITGAD \\
\hline AR & TSG101 \\
\hline MRE11A & RAD50 \\
\hline HLA & KLRD1 \\
\hline C8A & C8G \\
\hline AKAP6 & RYR2 \\
\hline CCDC85B & TEAD4 \\
\hline CRMP1 & UBE2B \\
\hline RXRA & VDR \\
\hline PSMB4 & SMAD1 \\
\hline LPL & VLDLR \\
\hline PHB & RB1 \\
\hline HSF1 & PRKDC \\
\hline FEZ1 & FEZ2 \\
\hline HLA & HLA \\
\hline MUC1 & SRC \\
\hline CSNK1A1 & OCLN \\
\hline
\end{tabular}




\begin{tabular}{|c|c|}
\hline BIN1 & MYC \\
\hline GFAP & PDLIM7 \\
\hline CSNK2B & CSNK2B \\
\hline MCM7 & UBE3A \\
\hline CD63 & HLA \\
\hline CDK4 & CDKN2B \\
\hline EEF1A1 & MAD2L1BP \\
\hline CREBBP & EGR1 \\
\hline DAG1 & DMD \\
\hline ESR1 & TDG \\
\hline FSCN1 & NGFR \\
\hline BMPR2 & BMPR2 \\
\hline DUSP6 & MAPK1 \\
\hline DUSP6 & MDFI \\
\hline EIF2AK2 & PTGES3 \\
\hline GEM & PDLIM7 \\
\hline CCL5 & CCR3 \\
\hline ATR & BRCA1 \\
\hline BRCA1 & RAD51 \\
\hline PEBP1 & RAF1 \\
\hline APOA1 & LCAT \\
\hline POLD2 & POLD3 \\
\hline SIAH1 & SLC9A3R2 \\
\hline CSTA & CSTA \\
\hline NCOA6 & NCOR2 \\
\hline CDC20 & MAD2L1 \\
\hline LCK & RAF1 \\
\hline CASP9 & DCC \\
\hline LYN & PLCG2 \\
\hline APC & CTNNB1 \\
\hline LMO2 & MAPRE3 \\
\hline NCOR2 & RXRA \\
\hline TAF4 & TAF6 \\
\hline TAF1 & TAF10 \\
\hline ID2 & RBL2 \\
\hline EGFR & NCK1 \\
\hline SKI & SMAD2 \\
\hline PRPS1 & PRPS1 \\
\hline EPOR & VAV1 \\
\hline BCL2L1 & RAD9A \\
\hline CRHBP & UCN \\
\hline FXR2 & PAICS \\
\hline PIK3R1 & TYRO3 \\
\hline ACVR1 & ENG \\
\hline ACVR1B & SMAD2 \\
\hline EED & HDAC1 \\
\hline SMAD4 & TFE3 \\
\hline
\end{tabular}




\begin{tabular}{|c|c|}
\hline CRMP1 & SERPINB9 \\
\hline ADAM15 & TRIP13 \\
\hline CTNNB1 & DVL1 \\
\hline 2-Sep & 6-Sep \\
\hline SREBF1 & UBE2I \\
\hline KTN1 & RHOG \\
\hline AQP1 & CCDC85B \\
\hline GNAO1 & RGS4 \\
\hline АСТВ & SMAD3 \\
\hline CCND3 & NCOA2 \\
\hline MYB & $\mathrm{NCL}$ \\
\hline PDGFRB & PLCG1 \\
\hline HLA & LILRB1 \\
\hline CLU & LEP \\
\hline FKBP4 & IRF4 \\
\hline ANXA5 & SUPT4H1 \\
\hline MAPK1 & PTPRR \\
\hline IL17A & IL17A \\
\hline BMPR1B & GDF5 \\
\hline APCS & FN1 \\
\hline GPNMB & SMAD4 \\
\hline SMAD7 & TGFBR2 \\
\hline MED1 & MED12 \\
\hline CD5 & RASA1 \\
\hline DLG2 & GRIN2A \\
\hline EEF1B2 & TIMP1 \\
\hline APC & TUBA4A \\
\hline PTPN6 & TYK2 \\
\hline ERBB3 & PTK6 \\
\hline NR5A1 & PITX1 \\
\hline BRCA1 & SMARCA4 \\
\hline REL & RELA \\
\hline EP300 & TCF12 \\
\hline EP300 & TDG \\
\hline CASP8 & MAP3K14 \\
\hline TNNC1 & TNNI2 \\
\hline KRT9 & YWHAQ \\
\hline ERBB4 & STAT5B \\
\hline BTK & PRKD1 \\
\hline RAD52 & SUMO1 \\
\hline SNAP25 & VAMP7 \\
\hline SRP19 & TNPO1 \\
\hline ELK1 & UBE2I \\
\hline FN1 & LPA \\
\hline MAGI1 & NET1 \\
\hline PRKCZ & SQSTM1 \\
\hline$A R$ & MED1 \\
\hline
\end{tabular}




\begin{tabular}{|c|c|}
\hline EPAS1 & SMAD3 \\
\hline TNNI3 & TNNT2 \\
\hline ARHGDIA & JUP \\
\hline CX3CL1 & CX3CL1 \\
\hline CCDC85B & ZNF250 \\
\hline ITSN2 & PTN \\
\hline PAM & $\mathrm{TSC} 2$ \\
\hline CD36 & YES1 \\
\hline CDC34 & CUL1 \\
\hline CREBBP & MYC \\
\hline COPS6 & SERPINB9 \\
\hline NFKBIA & SKP1 \\
\hline $\mathrm{BCL} 2$ & PMAIP1 \\
\hline EZH2 & POLA2 \\
\hline CCNA2 & CDK2 \\
\hline FUS & RELA \\
\hline E2F4 & RBL1 \\
\hline EXOSC2 & KHSRP \\
\hline ATXN1 & KCNAB2 \\
\hline CCNA2 & CDC6 \\
\hline DNMT1 & PCNA \\
\hline ANXA3 & TP53 \\
\hline IL9R & JAK1 \\
\hline RAF1 & SFN \\
\hline RET & SRC \\
\hline COPS8 & GPS1 \\
\hline FLT4 & VEGFC \\
\hline KCNJ12 & SNTB1 \\
\hline CD22 & PLCG1 \\
\hline SMN1 & SNRPB \\
\hline BATF & JUNB \\
\hline IL6ST & VAV1 \\
\hline ATF2 & CEBPA \\
\hline CASP3 & CASP3 \\
\hline CASP10 & CASP4 \\
\hline DCTN2 & GADD45A \\
\hline CUX1 & $\mathrm{RB1}$ \\
\hline ABL1 & HCK \\
\hline CCDC85B & SIX1 \\
\hline MDFI & PIN1 \\
\hline CDC25B & YWHAH \\
\hline HLA & LILRB1 \\
\hline CAV1 & S1PR1 \\
\hline ADRBK1 & $\mathrm{RHO}$ \\
\hline HSP90AA1 & PIM1 \\
\hline TAF6 & TAF9 \\
\hline SHC1 & SRC \\
\hline
\end{tabular}




\begin{tabular}{|c|c|}
\hline CYCS & UQCRC1 \\
\hline BAT3 & RAB8A \\
\hline NRIP1 & RARA \\
\hline TAF12 & TAF9 \\
\hline BMP2 & MGP \\
\hline CUL1 & NFKBIA \\
\hline TNNT1 & TNNT1 \\
\hline NUP214 & ZFP36 \\
\hline CAV1 & GRK5 \\
\hline GOLGA1 & RAB6A \\
\hline CRYAB & CRYAB \\
\hline NCOA2 & NKX2 \\
\hline CXCR4 & PTPN6 \\
\hline RALGDS & RIT1 \\
\hline CD40 & MS4A1 \\
\hline FBL & MAP3К14 \\
\hline $\mathrm{FBL}$ & PRMT1 \\
\hline ESR1 & PHB2 \\
\hline PDIA3 & STAT3 \\
\hline NCOA4 & PPARG \\
\hline POLR2E & POLR2L \\
\hline EP300 & PAX6 \\
\hline МАР3К11 & RHOG \\
\hline PRKDC & XRCC6 \\
\hline ADD1 & ID2 \\
\hline MEF2A & THRA \\
\hline GARS & IARS \\
\hline CDC7 & CDKN2A \\
\hline ACTA1 & ACTA1 \\
\hline $\mathrm{APOH}$ & LRP2 \\
\hline CALCOCO2 & $\mathrm{CALCOCO} 2$ \\
\hline ITGA2 & MMP1 \\
\hline NCOR2 & SRF \\
\hline EIF2AK2 & NPM1 \\
\hline E4F1 & EHMT2 \\
\hline GRB2 & IQGAP1 \\
\hline PAK1 & PLCG1 \\
\hline EGR1 & RELA \\
\hline GNAZ & RGS19 \\
\hline FLT1 & PGF \\
\hline RAC1 & STAT3 \\
\hline MNAT1 & POU5F1 \\
\hline TYK2 & TYK2 \\
\hline ETV6 & FLI1 \\
\hline ILF3 & MAGEA11 \\
\hline ANKS1A & SFN \\
\hline AR & TP53 \\
\hline
\end{tabular}




\begin{tabular}{|c|c|}
\hline CTNNB1 & PITX2 \\
\hline FYN & PIK3R2 \\
\hline ATN1 & PLSCR1 \\
\hline PI4KB & YWHAQ \\
\hline EEF1D & KTN1 \\
\hline EPOR & PTPN6 \\
\hline TPH1 & YWHAZ \\
\hline CREBBP & KAT2B \\
\hline PML & TDG \\
\hline DIS3 & ZBTB17 \\
\hline NEDD9 & ZYX \\
\hline MAPT & STXBP1 \\
\hline HSPA5 & TSHR \\
\hline DVL1 & DVL1 \\
\hline DLG3 & GUCY1A2 \\
\hline GADD45A & RXRA \\
\hline CDC25C & PLK1 \\
\hline MVP & PTEN \\
\hline$A R$ & CALR \\
\hline ARHGEF16 & SFN \\
\hline TEK & TIE1 \\
\hline KPNA1 & RUNX1T1 \\
\hline FGA & THBS1 \\
\hline H2AFX & WRN \\
\hline BAD & BCL2A1 \\
\hline ANK1 & SLC4A1 \\
\hline DAB2 & LRP2 \\
\hline CD40 & TRAF6 \\
\hline STX16 & VAMP3 \\
\hline MARK3 & SFN \\
\hline FEZ1 & IMMT \\
\hline ATXN2 & SH3GL2 \\
\hline PIK3CA & PIK3R3 \\
\hline HSP90AA1 & MYC \\
\hline CASP8 & FAS \\
\hline ATXN1 & TRIM32 \\
\hline COPS6 & COPS8 \\
\hline KRT8 & PKP1 \\
\hline MYC & SP1 \\
\hline$A R$ & CTDSP2 \\
\hline NCOA1 & PRMT1 \\
\hline F13B & FGG \\
\hline CRK & DOCK1 \\
\hline FTL & TAF10 \\
\hline CREBBP & PTMA \\
\hline CNTFR & IL6ST \\
\hline BRCA1 & EP300 \\
\hline
\end{tabular}




\begin{tabular}{|c|c|}
\hline COPS6 & SNRPG \\
\hline CALM1 & SNCA \\
\hline SERPINB9 & XRCC6 \\
\hline ELMO1 & HCK \\
\hline CTNNB1 & GSK3B \\
\hline INSR & MAPK3 \\
\hline DLST & NAP1L1 \\
\hline BAT2 & FGA \\
\hline $\mathrm{C} 8 \mathrm{~A}$ & CD59 \\
\hline FYN & PRKCQ \\
\hline CFTR & STX1A \\
\hline PICALM & PLCG1 \\
\hline PML & RPL11 \\
\hline AKAP1 & PRKAR1B \\
\hline PGF & VEGFA \\
\hline GATA4 & NR5A1 \\
\hline RAF1 & RHEB \\
\hline CASP3 & SRP72 \\
\hline CD247 & STAT5B \\
\hline ID3 & TCF4 \\
\hline CRYAA & HSPB1 \\
\hline HNRNPC & PHKB \\
\hline SMAD7 & TTF1 \\
\hline ATN1 & WWP1 \\
\hline $\mathrm{CDH} 11$ & CTNNB1 \\
\hline CASP3 & MCL1 \\
\hline NEDD9 & PXN \\
\hline NFYA & NFYC \\
\hline CASP3 & HSPE1 \\
\hline KHDRBS1 & RASA1 \\
\hline CDK8 & MED21 \\
\hline AP2M1 & FXR2 \\
\hline HSP90AA1 & IKBKE \\
\hline RPS6KA1 & TSC2 \\
\hline TTK & TUBB \\
\hline CASP1 & EGFR \\
\hline COPS5 & UCHL1 \\
\hline FLNA & GP1BA \\
\hline POLR2A & SUPT5H \\
\hline ESR1 & HSPA4 \\
\hline KIF23 & YWHAB \\
\hline GTF2F1 & TAF1 \\
\hline KIF11 & KIF11 \\
\hline DRD4 & NCK1 \\
\hline KRT18 & YWHAZ \\
\hline ARHGEF7 & RAC1 \\
\hline CR2 & FCER2 \\
\hline
\end{tabular}




\begin{tabular}{|c|c|}
\hline SUMO1 & TNFRSF1A \\
\hline EXT1 & EXT2 \\
\hline $\mathrm{BCL} 2$ & BLK \\
\hline PSMD11 & PTN \\
\hline ACP1 & EPHA2 \\
\hline $\mathrm{CHM}$ & RAB6A \\
\hline SKIL & TDG \\
\hline MDK & PTPRZ1 \\
\hline PDLIM7 & RET \\
\hline FLII & TRAIP \\
\hline NXF1 & SFRS7 \\
\hline TUBB & YWHAZ \\
\hline C1QBP & MMP14 \\
\hline CSNK2A1 & MAZ \\
\hline NFYC & SMAD3 \\
\hline SMAD2 & ST13 \\
\hline CUL4A & DDB1 \\
\hline SKIL & SNX17 \\
\hline GNAS & GNAS \\
\hline CSF3R & $\mathrm{HCK}$ \\
\hline BRCA1 & JUNB \\
\hline DHX9 & NXF1 \\
\hline PKP4 & PSEN1 \\
\hline MDM4 & RB1 \\
\hline CYP2C9 & POR \\
\hline ARNT & PML \\
\hline BHMT & BHMT \\
\hline CDK2 & CKS1B \\
\hline ATF2 & FOS \\
\hline NFKB2 & NFKBIB \\
\hline AFAP1 & PRKCB \\
\hline NR3C1 & NR3C2 \\
\hline RBBP6 & YBX1 \\
\hline ID3 & MYF5 \\
\hline ITK & WAS \\
\hline SRC & TRAF6 \\
\hline BRCA1 & CREBBP \\
\hline COPS2 & COPS5 \\
\hline CTNNB1 & PTPN1 \\
\hline MYBL1 & $\mathrm{NCL}$ \\
\hline CD53 & CD9 \\
\hline ETS2 & ZMYND11 \\
\hline MAPKAPK2 & PHC2 \\
\hline HOXA9 & SMAD4 \\
\hline ADRM1 & PSMD2 \\
\hline ARL4D & EPRS \\
\hline GRIA3 & SDCBP \\
\hline
\end{tabular}




\begin{tabular}{|c|c|}
\hline EGF & ERBB3 \\
\hline CCL7 & CCL7 \\
\hline RB1 & THOC1 \\
\hline MCM6 & MCM6 \\
\hline SMARCA4 & SMARCC1 \\
\hline PPP2R1A & PPP2R5A \\
\hline EWSR1 & RPS15A \\
\hline ATXN1 & KIAA0174 \\
\hline KARS & PAFAH1B1 \\
\hline LMO1 & LYL1 \\
\hline DDX5 & NCOA2 \\
\hline CSNK2A1 & TSPY1 \\
\hline MAPK3 & RPS6KA1 \\
\hline KAT2B & NCOA1 \\
\hline LYN & TYK2 \\
\hline ARHGEF7 & PAK1 \\
\hline GALE & GALE \\
\hline EEF1G & RPS28 \\
\hline EPS8 & $\mathrm{SHB}$ \\
\hline NPAS2 & RARA \\
\hline GSTM3 & GSTM3 \\
\hline NEDD4 & SCNN1B \\
\hline STX4 & VAMP3 \\
\hline CBS & FXR2 \\
\hline CDC6 & МСM3 \\
\hline LCK & THY1 \\
\hline IL11 & MAGEA11 \\
\hline MNAT1 & TP53 \\
\hline RYR1 & S100A1 \\
\hline IFIT3 & IFIT3 \\
\hline EP300 & SOX9 \\
\hline TTR & VIM \\
\hline SHB & SRC \\
\hline FASLG & GRAP \\
\hline RBPMS & SF1 \\
\hline KHDRBS1 & SMAD2 \\
\hline FAP & PLAUR \\
\hline RAD9A & TOPBP1 \\
\hline $\mathrm{HBB}$ & $\mathrm{HP}$ \\
\hline KRT15 & KRT6B \\
\hline ID3 & TCF12 \\
\hline TAF5 & TBP \\
\hline MYOD1 & SRF \\
\hline BLM & SUMO2 \\
\hline DSC1 & DSG2 \\
\hline CCND1 & CDK6 \\
\hline NOVA1 & PCBP1 \\
\hline
\end{tabular}




\begin{tabular}{|c|c|}
\hline NTF3 & NTRK3 \\
\hline JUN & POU1F1 \\
\hline CREBBP & DAXX \\
\hline KRT15 & KRT20 \\
\hline CFLAR & RAF1 \\
\hline ELN & NID2 \\
\hline COMMD1 & NFKBIA \\
\hline LGALS3 & LGALS3BP \\
\hline PITX1 & RBPMS \\
\hline CD3E & $\mathrm{NCL}$ \\
\hline MCM2 & MCM2 \\
\hline CBX1 & PIM1 \\
\hline PEX13 & PEX13 \\
\hline CDH5 & CTNNA1 \\
\hline ATXN1 & NARS \\
\hline E2F4 & RBL2 \\
\hline NCK1 & WAS \\
\hline IER3 & PPP2R5B \\
\hline FBLN1 & NID1 \\
\hline ACOT7 & ATXN1 \\
\hline C19orf57 & TRIM23 \\
\hline CREBBP & FOXO4 \\
\hline KRT10 & SMAD3 \\
\hline CSNK2A1 & HCLS1 \\
\hline PLG & SERPINE1 \\
\hline A2M & MMP2 \\
\hline RELN & VLDLR \\
\hline NPAS2 & RXRA \\
\hline EIF3B & EIF3I \\
\hline FURIN & SERPINB8 \\
\hline DDX5 & $\mathrm{FBL}$ \\
\hline $\mathrm{CRH}$ & CRHR2 \\
\hline DAZAP2 & DAZAP2 \\
\hline ITSN1 & UNC119 \\
\hline CREBBP & RPS6KA3 \\
\hline CCNA1 & RB1 \\
\hline NEDD4 & UBE2D3 \\
\hline JAK1 & PTK2B \\
\hline AMFR & UBE2D2 \\
\hline MAPK8 & PAX2 \\
\hline SLC26A3 & SLC9A3R2 \\
\hline UBE2N & UBE2V2 \\
\hline AKT1 & HSP90AB1 \\
\hline EPS8 & ITGB5 \\
\hline PTPN6 & ZAP70 \\
\hline CDC25A & CDK2 \\
\hline HNF4A & SREBF2 \\
\hline
\end{tabular}




\begin{tabular}{|c|c|}
\hline CLK2 & PTPN1 \\
\hline BGN & TNF \\
\hline ABL1 & RIN1 \\
\hline PBXIP1 & TSNAX \\
\hline PAFAH1B1 & PAFAH1B3 \\
\hline LCK & ZAP70 \\
\hline IFNAR1 & STAT2 \\
\hline DCC & MAZ \\
\hline ARFIP2 & ITGB3BP \\
\hline MDK & $\mathrm{NCL}$ \\
\hline SYN1 & SYN2 \\
\hline FAS & SUMO1 \\
\hline PCNA & WRN \\
\hline DAB2 & SMAD3 \\
\hline PML & TP53 \\
\hline EP300 & MGMT \\
\hline TBP & TBP \\
\hline $\mathrm{CDH} 2$ & GNA13 \\
\hline ABLIM1 & KCNJ12 \\
\hline RHOA & TRIO \\
\hline AP3D1 & VAMP7 \\
\hline KLC1 & YWHAB \\
\hline RNF10 & SETDB1 \\
\hline CSNK2A1 & TOP1 \\
\hline HRAS & MAP2K1 \\
\hline CAPN2 & PTPN1 \\
\hline EGFR & PRKAR1A \\
\hline CD22 & INPP5D \\
\hline INS & NOV \\
\hline NME1 & SET \\
\hline PEG3 & USP7 \\
\hline FYN & HTR6 \\
\hline BAT2 & GANAB \\
\hline SMAD2 & ST5 \\
\hline PSMA1 & PSMA3 \\
\hline TP53 & YWHAZ \\
\hline CALR & F5 \\
\hline MAP2K6 & MAР3К5 \\
\hline GATA1 & LMO2 \\
\hline CLIP1 & IQGAP1 \\
\hline PRKACA & RELA \\
\hline EFNB1 & SRC \\
\hline TRIM28 & VIM \\
\hline FAS & RIPK1 \\
\hline BAG1 & VDR \\
\hline GHR & PTPN2 \\
\hline IL10RA & JAK1 \\
\hline
\end{tabular}




\begin{tabular}{|c|c|}
\hline SETDB1 & ZNF24 \\
\hline BLK & PLCG2 \\
\hline ARHGEF2 & RAC1 \\
\hline ESR1 & FOXO4 \\
\hline $\mathrm{EZH} 2$ & PSMB6 \\
\hline ACTA1 & CFL1 \\
\hline KIAA0182 & XRCC6 \\
\hline TNFRSF9 & TRAF1 \\
\hline NCOA6 & SMAD2 \\
\hline BCL2L1 & VDAC1 \\
\hline LYN & PTPN6 \\
\hline PSEN1 & PSEN1 \\
\hline HTT & TRIP10 \\
\hline F11 & GP1BA \\
\hline TP53BP2 & UNC119 \\
\hline NCK1 & WIPF1 \\
\hline ARHGEF1 & CD44 \\
\hline ID1 & MYF5 \\
\hline JUN & NRIP1 \\
\hline MYD88 & TLR4 \\
\hline IL2RB & STAT3 \\
\hline CSNK1A1 & YWHAZ \\
\hline NDN & NUBP1 \\
\hline TAF12 & TAF6 \\
\hline F8 & LMAN1 \\
\hline ID1 & TCF3 \\
\hline ATXN3 & RAD23A \\
\hline EWSR1 & MY01F \\
\hline HOXA9 & PBX3 \\
\hline IL2RB & STAT5A \\
\hline EIF2S2 & UNC119 \\
\hline BRD8 & RXRA \\
\hline CKMT2 & UNC119 \\
\hline FBLN1 & FGB \\
\hline FASLG & GRB2 \\
\hline B3GNTL1 & MPP3 \\
\hline TEC & VAV1 \\
\hline $\mathrm{CCNH}$ & MCM7 \\
\hline ANP32A & E4F1 \\
\hline MCM2 & MCM4 \\
\hline FGF2 & SDC2 \\
\hline CDK7 & ERCC3 \\
\hline GTF2E1 & TAF6 \\
\hline MCM4 & SSRP1 \\
\hline ERBB4 & MUC1 \\
\hline DNMT1 & HDAC2 \\
\hline FLNC & SGCD \\
\hline
\end{tabular}




\begin{tabular}{|c|c|}
\hline F2 & F5 \\
\hline $\mathrm{BCL} 2$ & RAD9A \\
\hline FUS & ILF3 \\
\hline $\mathrm{CDH} 1$ & CTNNB1 \\
\hline GHR & PTPN3 \\
\hline EPS15 & LAPTM5 \\
\hline GATA3 & LMO2 \\
\hline AR & COX5B \\
\hline ADRA2B & EIF2B1 \\
\hline ATXN1 & ATXN2L \\
\hline KPNA2 & PLAG1 \\
\hline MAX & $\mathrm{MSH} 2$ \\
\hline GRB2 & PTK2B \\
\hline GAPDH & PRDX1 \\
\hline ATP2B2 & DLG2 \\
\hline BAT3 & TOMM20 \\
\hline PSMA1 & VIM \\
\hline DUSP1 & MAPK1 \\
\hline PIK3R2 & SYK \\
\hline BRCA1 & RBBP7 \\
\hline GDI2 & RAB4A \\
\hline JUN & TSC22D3 \\
\hline DGKQ & RHOA \\
\hline CSNK2B & FGF2 \\
\hline DUSP4 & MAPK14 \\
\hline RARA & SP1 \\
\hline DOK1 & FGR \\
\hline TRIM21 & USP4 \\
\hline CREBBP & ETS1 \\
\hline VASP & ZYX \\
\hline GRB2 & PTPN1 \\
\hline DOCK1 & ELMO1 \\
\hline JAK3 & PTK2B \\
\hline CD59 & SMAD4 \\
\hline CD33 & PTPN11 \\
\hline JUN & NACA \\
\hline SNRPD3 & SNRPE \\
\hline ACTA1 & FSCN1 \\
\hline TP53 & TP53BP2 \\
\hline TERT & XRCC5 \\
\hline ERBB3 & NRG1 \\
\hline EEA1 & IGF1R \\
\hline DLG2 & KCNJ12 \\
\hline ATF4 & GPS2 \\
\hline DLG4 & MAP1A \\
\hline GRIA1 & PRKACA \\
\hline CREBBP & NCOA6 \\
\hline
\end{tabular}




\begin{tabular}{|c|c|}
\hline FXR2 & PCBD1 \\
\hline BCL2L1 & CFLAR \\
\hline CBL & SH3GL2 \\
\hline PSMD11 & TLE1 \\
\hline HLA & PDIA3 \\
\hline PFN2 & PTPRS \\
\hline GTF2B & NR5A1 \\
\hline DLG1 & GUCY1A2 \\
\hline COL2A1 & MAG \\
\hline CRYGC & CRYGC \\
\hline UBE2I & WT1 \\
\hline FYN & SHC1 \\
\hline PLK1 & PSMB5 \\
\hline CCL7 & MMP2 \\
\hline AMPH & CLTC \\
\hline SMAD2 & SMAD3 \\
\hline FKBP1A & TGFBR1 \\
\hline SNAP25 & SYT1 \\
\hline HNRNPF & NCBP2 \\
\hline SF3B3 & wWox \\
\hline HCK & WIPF1 \\
\hline CD3E & TOP2B \\
\hline NPR1 & NPR1 \\
\hline $\mathrm{CBL}$ & KIT \\
\hline ID1 & MYOD1 \\
\hline FN1 & PKD1 \\
\hline DLG1 & DLGAP1 \\
\hline FHL2 & PFKM \\
\hline ATXN1 & COIL \\
\hline INSR & MAD2L1 \\
\hline IGF1R & PTPN11 \\
\hline ACP5 & TNFSF10 \\
\hline ESR2 & NCOA1 \\
\hline NROB1 & NR5A1 \\
\hline PTN & TP53BP2 \\
\hline CTH & CTH \\
\hline CALM1 & RELA \\
\hline HNRNPL & HNRNPL \\
\hline CASP10 & CASP7 \\
\hline ANXA2 & CEACAM1 \\
\hline RFC1 & RFC2 \\
\hline ERCC3 & GTF2H4 \\
\hline GNB2L1 & PRKCA \\
\hline AP1B1 & ARF1 \\
\hline CUL2 & NEDD8 \\
\hline SLA & ZAP70 \\
\hline NR3C1 & YWHAH \\
\hline
\end{tabular}




\begin{tabular}{|c|c|}
\hline TPM2 & TPM2 \\
\hline KAT5 & MDM2 \\
\hline ATM & ATM \\
\hline TMPO & USP7 \\
\hline AXIN1 & CRMP1 \\
\hline NFE2L2 & PPARG \\
\hline S100B & S100B \\
\hline MSX2 & RUNX2 \\
\hline APC & JUP \\
\hline POLB & TLE1 \\
\hline TAF11 & TAF9 \\
\hline GATA4 & TBX5 \\
\hline BCL2L1 & BNIP1 \\
\hline CXCL1 & CXCL1 \\
\hline CDC25A & YWHAE \\
\hline ACTN1 & FXN \\
\hline FGF2 & RPS19 \\
\hline IL6ST & OSMR \\
\hline CCNA1 & CDK2 \\
\hline EGFR & ERBB2 \\
\hline ACPP & ACPP \\
\hline MUTYH & RPA1 \\
\hline MDM2 & UBE2A \\
\hline CACNA1A & CACNB4 \\
\hline KRT15 & PCM1 \\
\hline PPFIA1 & PTPRD \\
\hline SP1 & TP53 \\
\hline АСТВ & RAC2 \\
\hline KNG1 & PLG \\
\hline AKT1 & MAP2K4 \\
\hline CAV1 & PTPN6 \\
\hline AKT1 & RPS6KB1 \\
\hline ATXN2 & SH3GL3 \\
\hline CLNS1A & SNRPB \\
\hline DLD & OGDH \\
\hline FYN & SH3BP2 \\
\hline AMBP & FN1 \\
\hline C9 & CD59 \\
\hline SALL1 & UBE2I \\
\hline AR & JUN \\
\hline CRMP1 & PSMD11 \\
\hline KAT5 & TP53 \\
\hline CFTR & PRKCE \\
\hline POLR2E & POLR2H \\
\hline CD4 & PTPRC \\
\hline SYN2 & SYN2 \\
\hline MMP3 & TIMP1 \\
\hline
\end{tabular}




\begin{tabular}{|c|c|}
\hline EEF1A1 & PSMD11 \\
\hline MMP2 & TIMP3 \\
\hline FGF9 & FGF9 \\
\hline CASP8 & HIP1 \\
\hline ATN1 & GRN \\
\hline PSMC2 & PSMD2 \\
\hline UPP1 & VIM \\
\hline ETS1 & ETS2 \\
\hline FOXF2 & GTF2B \\
\hline DAB2 & GRB2 \\
\hline FADD & FASLG \\
\hline $\mathrm{CD} 2$ & CD5 \\
\hline MCM2 & ORC2L \\
\hline CBX1 & CBX5 \\
\hline DCTN1 & KIF11 \\
\hline CASR & FLNA \\
\hline NCBP1 & NCBP2 \\
\hline PLSCR1 & SMCP \\
\hline APBB1 & APLP2 \\
\hline LCP2 & LYN \\
\hline DSG2 & JUP \\
\hline SDC1 & SDCBP \\
\hline TRIM28 & TRIM28 \\
\hline UBE2L3 & UBE3A \\
\hline NR1H4 & RXRA \\
\hline SLC6A9 & STX1A \\
\hline EGR1 & TP53 \\
\hline NCOA1 & PPARG \\
\hline UPK1A & UPK2 \\
\hline IFI35 & NMI \\
\hline MARK3 & TTR \\
\hline IL1R2 & IL1RN \\
\hline CDK6 & MCM2 \\
\hline ARF1 & ARF1 \\
\hline E2F1 & MDM4 \\
\hline CCNE1 & CDK2 \\
\hline ERBB3 & GRB2 \\
\hline FLNC & SGCG \\
\hline HRAS & RALGDS \\
\hline GGH & GGH \\
\hline CBL & PDGFRA \\
\hline NMI & STAT3 \\
\hline FYB & VASP \\
\hline NUMB & PRKCZ \\
\hline RB1 & RBBP8 \\
\hline DES & NEB \\
\hline EEF1B2 & HARS \\
\hline
\end{tabular}




\begin{tabular}{|c|c|}
\hline MAPK14 & SMAD7 \\
\hline IL16 & IL16 \\
\hline FOXO1 & SMAD3 \\
\hline PSMB7 & PSMB7 \\
\hline ALCAM & CD6 \\
\hline HDAC1 & RBL1 \\
\hline LIMK1 & NRG1 \\
\hline ABL1 & NEDD9 \\
\hline ACVR2A & INHBB \\
\hline HTR1A & HTR1D \\
\hline RAF1 & VAV1 \\
\hline HSF1 & SMARCA4 \\
\hline SRRM2 & YWHAB \\
\hline GTF2B & NCOA1 \\
\hline BAT2 & QARS \\
\hline MAF & USF2 \\
\hline RAD51 & TP53 \\
\hline KIT & STAT1 \\
\hline $\mathrm{BCL} 2$ & BNIP1 \\
\hline TNFRSF14 & VIM \\
\hline PSEN2 & SRI \\
\hline EPRS & HSP90AA1 \\
\hline HSF1 & STAT1 \\
\hline HNRNPD & SFN \\
\hline HRAS & RGL2 \\
\hline CEACAM1 & PTPN11 \\
\hline PRKDC & TP53 \\
\hline CD1D & PTPRC \\
\hline CYP11A1 & SMAD2 \\
\hline FN1 & TGFBI \\
\hline DEK & TFAP2A \\
\hline RBL1 & SP1 \\
\hline CSNK2A2 & KLF1 \\
\hline LYN & SHC1 \\
\hline CBLB & KIT \\
\hline PRMT1 & S100A8 \\
\hline MAX & MAX \\
\hline ADAM17 & DLG1 \\
\hline PTH1R & YWHAH \\
\hline INPPL1 & SHC1 \\
\hline CACNB4 & PTN \\
\hline KAT5 & LMNA \\
\hline HSPB1 & MAPKAPK2 \\
\hline MMP9 & THBS1 \\
\hline GZMA & SET \\
\hline COX17 & TP53 \\
\hline AP2M1 & TBC1D5 \\
\hline
\end{tabular}




\begin{tabular}{|c|c|}
\hline ATF6 & GTF2I \\
\hline BAT3 & RCN2 \\
\hline NCOA2 & PPARG \\
\hline CD48 & LCK \\
\hline LAPTM5 & TNFAIP3 \\
\hline NME1 & NME4 \\
\hline $\mathrm{KCNH} 2$ & $\mathrm{RHOH}$ \\
\hline LUM & MMP14 \\
\hline AKT2 & GSK3B \\
\hline $\mathrm{BCL} 2$ & RRAS \\
\hline ISL1 & LMO2 \\
\hline FHL2 & TTN \\
\hline NFKB1 & STAT6 \\
\hline CBL & HCK \\
\hline ADCYAP1R1 & CALM1 \\
\hline DSC2 & DSG1 \\
\hline ATN1 & MDFI \\
\hline TRIP10 & WAS \\
\hline F11 & SERPINA5 \\
\hline КАТ2B & NCOA4 \\
\hline RPA2 & UNG \\
\hline ITGB1 & TGOLN2 \\
\hline POLR2C & TAF15 \\
\hline LPL & RPL18A \\
\hline ARR3 & RNASE6 \\
\hline E2F4 & SMAD3 \\
\hline GRM3 & PPM1A \\
\hline HNRNPC & HNRNPC \\
\hline CSNK2A1 & FGF2 \\
\hline DNM1 & NCK1 \\
\hline ERCC4 & SPTAN1 \\
\hline DSC1 & PKP2 \\
\hline DLG4 & GRIN2A \\
\hline BMI1 & KAT5 \\
\hline PRDM2 & RB1 \\
\hline CASP8 & MAPK1 \\
\hline ANXA2 & PCNA \\
\hline MDFI & NR1H2 \\
\hline E2F1 & SP3 \\
\hline BRCA1 & CCNA2 \\
\hline CIRBP & RBMX \\
\hline CSTA & CTSB \\
\hline CCL3 & CCR5 \\
\hline RAD51 & RAD52 \\
\hline CBLB & SYK \\
\hline GAPDH & PGK1 \\
\hline СТВP1 & HDAC1 \\
\hline
\end{tabular}




\begin{tabular}{|c|c|}
\hline HDAC1 & IKZF1 \\
\hline NR5A1 & sox9 \\
\hline FN1 & ITGB6 \\
\hline PEX12 & PEX19 \\
\hline AKT1 & $\mathrm{EZH} 2$ \\
\hline E2F3 & RB1 \\
\hline POLR2E & POLR2G \\
\hline PTN & PTPRZ1 \\
\hline ANKRD1 & TTN \\
\hline STX4 & VAMP7 \\
\hline CSDA & MAPK1 \\
\hline GYPC & PDIA3 \\
\hline PTPN1 & STAT5A \\
\hline AR & IL6ST \\
\hline $\mathrm{A} 2 \mathrm{M}$ & KLK3 \\
\hline SPIB & TBP \\
\hline GATA4 & NKX2 \\
\hline NR2F6 & THRB \\
\hline MARK3 & PKP2 \\
\hline KEAP1 & MYO7A \\
\hline CDK4 & NCOA2 \\
\hline IGBP1 & PPP4C \\
\hline MC5R & POMC \\
\hline LYN & TRIP10 \\
\hline CBX5 & LBR \\
\hline POLR2H & POLR2K \\
\hline KRT1 & MBL2 \\
\hline HRAS & TIAM1 \\
\hline CD93 & KNG1 \\
\hline EGFR & PTPN11 \\
\hline CA2 & HSPD1 \\
\hline PPP2R1A & SMAD3 \\
\hline UBE2I & UNC119 \\
\hline ITSN1 & WASL \\
\hline IK & KAT5 \\
\hline EIF4G3 & MKNK1 \\
\hline B2M & BAT3 \\
\hline LSM1 & NARS \\
\hline CSK & EGFR \\
\hline PRKCl & SQSTM1 \\
\hline PECAM1 & YES1 \\
\hline NFATC4 & YWHAZ \\
\hline GATA2 & ZBTB16 \\
\hline HSPA1A & YWHAB \\
\hline CALCOCO2 & ZNF638 \\
\hline PTPN3 & SFN \\
\hline NDN & NDN \\
\hline
\end{tabular}




\begin{tabular}{|c|c|}
\hline TAX1BP3 & TCEA2 \\
\hline SMARCB1 & XPO1 \\
\hline$A R$ & RB1 \\
\hline EP300 & SP3 \\
\hline RTN1 & UGCG \\
\hline AXIN1 & SH3GL1 \\
\hline APBA2 & LRP1 \\
\hline AGTR1 & GRK5 \\
\hline EGF & VTN \\
\hline ATN1 & ZMYND8 \\
\hline BCL2L1 & BNIP3 \\
\hline ATP2A2 & S100A1 \\
\hline CSTA & $\mathrm{CTSH}$ \\
\hline ARPC1B & PAK1 \\
\hline ALK & SHC3 \\
\hline ACTA1 & TMSB4X \\
\hline EIF2S2 & ZBTB16 \\
\hline ACP5 & EGR2 \\
\hline $\mathrm{AMPH}$ & DNM1 \\
\hline CCDC106 & TP53 \\
\hline $\mathrm{NCL}$ & TOP1 \\
\hline GATA3 & LMO1 \\
\hline FKBP1B & RYR2 \\
\hline CTTN & DNM2 \\
\hline PTN & RIT1 \\
\hline FOXF2 & TBP \\
\hline EGFR & MAP3К14 \\
\hline CSNK2B & TOP2B \\
\hline CARS & EEF1G \\
\hline TAC1 & TACR2 \\
\hline F10 & PRKAB1 \\
\hline ANG & PTEN \\
\hline PTPRS & UNC119 \\
\hline CDKN1A & МАР3К5 \\
\hline GRB2 & WAS \\
\hline REG3A & SDC2 \\
\hline AR & SRY \\
\hline APEX1 & HSPA1A \\
\hline HDAC1 & HIF1A \\
\hline SF3A1 & WWOX \\
\hline CSF2RB & PTPN11 \\
\hline ARF3 & KIF23 \\
\hline EGF & PIK3R2 \\
\hline BAT2 & HNRNPM \\
\hline EZR & NF2 \\
\hline PTPN21 & SRC \\
\hline $\mathrm{HCK}$ & PECAM1 \\
\hline
\end{tabular}




\begin{tabular}{|c|c|}
\hline CoX17 & UNC119 \\
\hline DLG4 & FZD2 \\
\hline F10 & SERPINB8 \\
\hline $\mathrm{EZH} 2$ & SMS \\
\hline S100A12 & S100A12 \\
\hline FASTK & RBPMS \\
\hline JUN & RUNX1 \\
\hline FBP1 & FBP1 \\
\hline ATN1 & LYST \\
\hline CD226 & ITGB2 \\
\hline FANCC & SPTAN1 \\
\hline EZR & MSN \\
\hline PDGFRB & S1PR1 \\
\hline DNM2 & SRC \\
\hline ID3 & MYF6 \\
\hline DNMT1 & HDAC1 \\
\hline BNIP3 & TMEM11 \\
\hline JUNB & MAPK8 \\
\hline CREBBP & NFE2 \\
\hline PCBP1 & РCBP2 \\
\hline BLK & CD79A \\
\hline ARAF & CSNK2B \\
\hline $\mathrm{BCL} 2$ & MAPK8 \\
\hline CBL & MST1R \\
\hline IRAK1 & TRAF6 \\
\hline EIF5A & XPO1 \\
\hline EZR & PIK3R1 \\
\hline SUMO1 & TP53 \\
\hline EPS8 & SHC1 \\
\hline CBLB & ZAP70 \\
\hline CRKL & TYK2 \\
\hline CD46 & SRC \\
\hline ID2 & TCF12 \\
\hline E2F1 & NCOA6 \\
\hline CSF2RB & SHC1 \\
\hline ERCC1 & ERCC4 \\
\hline ESR1 & RNF4 \\
\hline PSMD11 & ZBTB16 \\
\hline FYN & SNCA \\
\hline BCAT1 & SMAD5 \\
\hline NKX3 & SRF \\
\hline CDK4 & RB1 \\
\hline COPS6 & EMD \\
\hline EEF1A1 & SSR1 \\
\hline FXYD3 & NR4A1 \\
\hline GATA2 & PML \\
\hline TNFAIP3 & TRAF6 \\
\hline
\end{tabular}




\begin{tabular}{|c|c|}
\hline POLR2C & SMARCC2 \\
\hline CDK4 & MCM2 \\
\hline BCL3 & HDAC1 \\
\hline CSTF2 & FEZ1 \\
\hline FGFR3 & RPL8 \\
\hline PAFAH1B2 & RPLPO \\
\hline MDM2 & USP7 \\
\hline HOXB6 & KRT15 \\
\hline HDAC1 & RFC1 \\
\hline ANXA2 & PLAT \\
\hline BRD2 & E2F1 \\
\hline PSMA2 & PSMA4 \\
\hline FSHR & GRK4 \\
\hline EEF1A1 & LAMA4 \\
\hline MAPK1 & YBX1 \\
\hline KRT15 & PRPH \\
\hline AES & GTF2E1 \\
\hline ADAM17 & TIMP3 \\
\hline POLA1 & TP53 \\
\hline CASP10 & CASP3 \\
\hline PRKDC & XRCC5 \\
\hline NEDD4 & SGK1 \\
\hline IL5RA & UNC119 \\
\hline HIVEP1 & SMAD3 \\
\hline MSN & TSC1 \\
\hline GTF2B & PSMC2 \\
\hline PSMD6 & TRAF6 \\
\hline GRB2 & PIK3R1 \\
\hline RB1 & RBBP4 \\
\hline NUCB1 & PTGS1 \\
\hline EHMT2 & LMO2 \\
\hline EP300 & RELA \\
\hline KIAA0182 & RBPMS \\
\hline RDBP & VAMP3 \\
\hline SGK1 & SLC9A3R2 \\
\hline CD79A & CD79B \\
\hline CREBBP & NFATC4 \\
\hline HDAC1 & RUNX1T1 \\
\hline SLC2A1 & STOM \\
\hline $\mathrm{BCL} 2$ & FKBP8 \\
\hline FHL2 & ITGB2 \\
\hline MAP3К14 & TRAF6 \\
\hline BRCA1 & CSNK2B \\
\hline TRO & USP7 \\
\hline GM2A & HEXA \\
\hline REL & SP1 \\
\hline ARHGDIB & $\mathrm{RHOH}$ \\
\hline
\end{tabular}




\begin{tabular}{|c|c|}
\hline NFKB1 & NFKBIA \\
\hline FOSL1 & JUNB \\
\hline VAV1 & XRCC6 \\
\hline CD72 & PTPN6 \\
\hline $\mathrm{CDH} 5$ & CTNNB1 \\
\hline NR2F2 & NR2F6 \\
\hline ATXN1 & DMPK \\
\hline CSE1L & PPP5C \\
\hline CALR & SLC6A4 \\
\hline ASPA & ASPA \\
\hline NEDD9 & PTK2B \\
\hline DOK1 & SHC1 \\
\hline NFKBIB & POLR2H \\
\hline$A R$ & CASP8 \\
\hline CRK & ERBB4 \\
\hline OGG1 & XRCC1 \\
\hline EPOR & LYN \\
\hline DGKZ & SNTB1 \\
\hline AKT1 & TSC1 \\
\hline MEOX2 & RND2 \\
\hline E2F3 & MYBL2 \\
\hline JUN & JUN \\
\hline GCK & GCKR \\
\hline ATN1 & CACNB1 \\
\hline PKM2 & RAF1 \\
\hline UCP2 & YWHAZ \\
\hline $\mathrm{GC}$ & LRP2 \\
\hline ODC1 & ODC1 \\
\hline EP300 & RUNX2 \\
\hline BRAF & PRKCE \\
\hline CCL23 & CCR1 \\
\hline PXN & RASA1 \\
\hline EYA2 & SIX1 \\
\hline ITSN1 & SNAP25 \\
\hline TOP1 & UBE2I \\
\hline GAPDH & PAFAH1B3 \\
\hline CDC25C & NEDD4 \\
\hline ESR2 & SRC \\
\hline COIL & NOLC1 \\
\hline CDKN1A & PIM1 \\
\hline JAG1 & NOTCH3 \\
\hline$A R$ & NCOA2 \\
\hline CREBBP & STAT1 \\
\hline VDR & ZBTB16 \\
\hline BAK1 & $\mathrm{BCL} 2$ \\
\hline PTPN6 & ROS1 \\
\hline MAPK1 & RPS6KA1 \\
\hline
\end{tabular}




\begin{tabular}{|c|c|}
\hline GTF2H1 & GTF2H2 \\
\hline ITGAD & VCAM1 \\
\hline RAB4A & RABEP1 \\
\hline DSG1 & JUP \\
\hline DOK1 & PLCG1 \\
\hline LRPAP1 & SORL1 \\
\hline ATP6V0D1 & ATXN1 \\
\hline GNA13 & PPP5C \\
\hline NEDD9 & RAPGEF1 \\
\hline NPM1 & TP53 \\
\hline COL17A1 & DST \\
\hline EEF1G & MVD \\
\hline NTF4 & NTRK2 \\
\hline BCAP31 & VAMP3 \\
\hline ARHGEF7 & GIT2 \\
\hline CD247 & JAK3 \\
\hline HERC1 & PKM2 \\
\hline$A R$ & NCOR2 \\
\hline МАР3К14 & MAP3К8 \\
\hline CDK5 & TP53 \\
\hline AP3B1 & AP3S2 \\
\hline FBLN1 & FN1 \\
\hline NFKB2 & RPL6 \\
\hline NCOA2 & RXRA \\
\hline ERBB2 & MATK \\
\hline LCP2 & VAV1 \\
\hline MLH1 & PMS1 \\
\hline CEBPA & SPI1 \\
\hline $\mathrm{NCL}$ & NR3C1 \\
\hline GAD1 & PRKCE \\
\hline ORC1L & ORC2L \\
\hline GHR & TYK2 \\
\hline C1QBP & PRKCD \\
\hline DMD & SNTB2 \\
\hline PSMC5 & PSMC6 \\
\hline ACTN2 & PDLIM1 \\
\hline SNRPE & SNRPF \\
\hline NCOA1 & NR4A1 \\
\hline GATA2 & POU1F1 \\
\hline COL2A1 & ITGA2B \\
\hline ATXN1 & CST3 \\
\hline CALM1 & TCF3 \\
\hline ORC2L & RPA1 \\
\hline GTF2F2 & HTATSF1 \\
\hline PAPPA & SKIL \\
\hline RGL2 & UNC119 \\
\hline NR3C1 & TRIM28 \\
\hline
\end{tabular}




\begin{tabular}{|c|c|}
\hline GNB2 & GNGT1 \\
\hline ABL2 & RIN1 \\
\hline DUSP1 & MAPK14 \\
\hline RIPK1 & RIPK1 \\
\hline PNO1 & RXRG \\
\hline COPB2 & PRKCE \\
\hline RAC1 & RPS6KB1 \\
\hline CDC27 & SMAD2 \\
\hline KLF9 & PGR \\
\hline CDC34 & CDC34 \\
\hline E2F4 & RB1 \\
\hline AGER & S100B \\
\hline FMR1 & $\mathrm{NCL}$ \\
\hline MARK3 & PRKCQ \\
\hline LCK & SQSTM1 \\
\hline GAPDH & KAT5 \\
\hline F2 & SERPINB6 \\
\hline APCS & APCS \\
\hline CDKN1A & STAT3 \\
\hline TNFRSF1A & TRADD \\
\hline ATN1 & BAT2 \\
\hline EEF1G & PSMD11 \\
\hline CASP8 & CFLAR \\
\hline PSMC5 & RORA \\
\hline CLNS1A & ITGA2B \\
\hline LAMA4 & UNC119 \\
\hline FES & STAT3 \\
\hline DOCK2 & RAC1 \\
\hline DLG4 & FYN \\
\hline LRP8 & RELN \\
\hline BMI1 & BMI1 \\
\hline RELB & SMARCC1 \\
\hline SERTAD2 & TRIM28 \\
\hline DAXX & ETS1 \\
\hline BAT3 & REG1B \\
\hline RUNX2 & SMAD2 \\
\hline BCL3 & COPS5 \\
\hline PAFAH1B1 & PAFAH1B1 \\
\hline EEF1A1 & RFC5 \\
\hline PLCB3 & SLC9A3R2 \\
\hline RGS3 & YWHAB \\
\hline $\mathrm{CSH} 1$ & PTPN12 \\
\hline RPA1 & TP53 \\
\hline COPS6 & PSMD11 \\
\hline NMT1 & TP53 \\
\hline NFKB2 & RELB \\
\hline LMO2 & NHLH1 \\
\hline
\end{tabular}




\begin{tabular}{|c|c|}
\hline HSPA5 & KRT8 \\
\hline RB1 & SP1 \\
\hline PRKCD & STAT1 \\
\hline PSMD11 & PTPRK \\
\hline PCGF2 & RNF2 \\
\hline CHGB & OGG1 \\
\hline IRF2 & IRF8 \\
\hline CALD1 & SHC1 \\
\hline AKT1 & SMAD2 \\
\hline IL1RL1 & TMED1 \\
\hline BMPR1B & TRAF6 \\
\hline TANK & TRAF3 \\
\hline BAK1 & BAK1 \\
\hline DLG4 & DLGAP1 \\
\hline SUMO1 & TOP2A \\
\hline CEBPA & CTNNB1 \\
\hline PDHB & PDHX \\
\hline RBM4 & SMAD5 \\
\hline PIN1 & TP53 \\
\hline MARS & PPM1F \\
\hline CASP10 & FASLG \\
\hline BAT3 & PTH \\
\hline ABL1 & MUC1 \\
\hline ACTA1 & GSN \\
\hline EEF1A1 & RAB27A \\
\hline NCOA6 & NR1H2 \\
\hline CCNE1 & COIL \\
\hline CCNE1 & CDKN1A \\
\hline CSF1R & LYN \\
\hline S100A10 & S100A10 \\
\hline EP300 & JUN \\
\hline TAF11 & TAF13 \\
\hline ANXA5 & COL2A1 \\
\hline MAGEA12 & STAT5A \\
\hline NFATC1 & PIM1 \\
\hline UCP2 & YWHAB \\
\hline CDC25A & PIM1 \\
\hline PMAIP1 & ZBTB16 \\
\hline $\mathrm{A} 2 \mathrm{M}$ & LCAT \\
\hline KRT8 & PLAT \\
\hline PPP2CA & RELA \\
\hline NCOA6 & SMAD3 \\
\hline HMGB1 & HOXD9 \\
\hline DDX1 & HNRNPK \\
\hline RNF5 & UBE2D3 \\
\hline ARF6 & EXOC5 \\
\hline ADORA2A & DRD2 \\
\hline
\end{tabular}




\begin{tabular}{|c|c|}
\hline H2AFX & TP53BP1 \\
\hline POLR2K & POLR2K \\
\hline PFN2 & RAP1GAP \\
\hline CDK2 & LYN \\
\hline COIL & TFCP2 \\
\hline LIMK1 & PAK1 \\
\hline EWSR1 & RBPMS \\
\hline CHD8 & RBBP5 \\
\hline PHB & RBL1 \\
\hline МАРЗКЗ & YWHAQ \\
\hline RBL1 & SMAD3 \\
\hline AKAP9 & CSNK1D \\
\hline CX3CL1 & CX3CR1 \\
\hline VEGFC & VEGFC \\
\hline ATF1 & SPI1 \\
\hline ITGB1 & LGALS8 \\
\hline AP2M1 & RALBP1 \\
\hline AKAP5 & PPP3CA \\
\hline MAPK1 & MBP \\
\hline RELA & RELB \\
\hline ANGPT1 & TEK \\
\hline NEDD8 & TP53 \\
\hline DNM1 & DNM1 \\
\hline CSNK2A1 & OGT \\
\hline MAGI1 & SFN \\
\hline DAG1 & SRC \\
\hline GRB2 & PDGFRB \\
\hline FLNB & GP1BA \\
\hline EEF1G & FOXG1 \\
\hline $\mathrm{F} 2$ & SERPING1 \\
\hline CUX1 & KAT2B \\
\hline NR3C1 & TP53 \\
\hline HTT & UBE2K \\
\hline PSMD11 & SMAD4 \\
\hline IER3 & MAPK3 \\
\hline TOPBP1 & TOPBP1 \\
\hline SKI & SKIL \\
\hline GTF2F2 & POLR2E \\
\hline TJP2 & YWHAB \\
\hline PRKAR1A & PRKAR1B \\
\hline BAK1 & VDAC2 \\
\hline NUP153 & TPR \\
\hline CITED1 & CREBBP \\
\hline EP300 & ESR1 \\
\hline ARHGAP11A & SFN \\
\hline ARHGEF2 & PAK1 \\
\hline IMMT & PPOX \\
\hline
\end{tabular}




\begin{tabular}{|c|c|}
\hline CASP3 & CASP8 \\
\hline ARHGEF12 & RHOA \\
\hline SP1 & SP3 \\
\hline BLK & UBE3A \\
\hline CFDP1 & SMAD3 \\
\hline CALM1 & CAMK $2 \mathrm{G}$ \\
\hline AES & RPL18A \\
\hline CXCL11 & CXCR3 \\
\hline CTNNB1 & DSC3 \\
\hline ARAF & ASS1 \\
\hline $\mathrm{ABI} 2$ & ABL1 \\
\hline HNRNPK & PCBP1 \\
\hline EPB41 & TJP2 \\
\hline $\mathrm{CDH} 1$ & GNA13 \\
\hline IRS1 & SFN \\
\hline DAZAP2 & RPS27A \\
\hline LCP2 & SHB \\
\hline CBLB & SHC1 \\
\hline U2AF2 & WT1 \\
\hline ACTC1 & AFAP1 \\
\hline NCOA1 & NR3C1 \\
\hline HNRNPK & SRC \\
\hline ABL1 & DOK1 \\
\hline IL6 & ZBTB16 \\
\hline POLR2C & POLR2F \\
\hline EEF1G & SAT1 \\
\hline PKN1 & PLD1 \\
\hline BTK & CAV1 \\
\hline ERBB2 & ERBB2 \\
\hline ABL1 & RAD51 \\
\hline BRCA2 & RAD51 \\
\hline CD19 & CR2 \\
\hline CAV1 & EDNRB \\
\hline BAG1 & $\mathrm{BCL} 2$ \\
\hline CREBBP & TRIP4 \\
\hline BRCA1 & NMI \\
\hline CREBBP & JUN \\
\hline ICAM5 & PSEN1 \\
\hline MDM2 & MDM4 \\
\hline CPSF6 & wWox \\
\hline GGA3 & IGF2R \\
\hline EGFR & GRB14 \\
\hline LCP2 & PIK3R1 \\
\hline KPNA3 & KPNB1 \\
\hline APCS & FCGR1A \\
\hline CDC34 & CDK9 \\
\hline PRKDC & RPA1 \\
\hline
\end{tabular}




\begin{tabular}{|c|c|}
\hline CHN1 & RPS3A \\
\hline IRS1 & JAK3 \\
\hline LRP2 & RBP1 \\
\hline CD19 & CD79B \\
\hline MAPK1 & NEK2 \\
\hline GLUL & GLUL \\
\hline $\mathrm{TSC} 2$ & YWHAB \\
\hline CREBBP & NR3C1 \\
\hline EIF3A & EIF3B \\
\hline MCM7 & RB1 \\
\hline MSX1 & TBP \\
\hline POLA1 & RB1 \\
\hline$A R$ & RAD9A \\
\hline CRKL & GRB2 \\
\hline HDAC1 & NCOR2 \\
\hline CTNNB1 & ESR1 \\
\hline EPOR & SHC1 \\
\hline CALM1 & VIPR1 \\
\hline PIM1 & SND1 \\
\hline TRIM23 & TRIM29 \\
\hline PIN1 & POLR2A \\
\hline HDAC2 & MTA1 \\
\hline ARR3 & CSNK $2 A$ \\
\hline FYN & PLCG2 \\
\hline SPI1 & TBP \\
\hline CDC25C & YWHAB \\
\hline ELN & FBLN2 \\
\hline FOS & JUND \\
\hline $\mathrm{BCL} 2$ & TP53BP2 \\
\hline CSF2RB & GNB2L1 \\
\hline CCDC85B & KRT20 \\
\hline STX4 & VAMP2 \\
\hline HLA & MBP \\
\hline BDNF & NTRK2 \\
\hline PRKCZ & SRC \\
\hline NTRK2 & SHC1 \\
\hline STAT1 & STAT1 \\
\hline SPIB & SPIB \\
\hline HNF4A & MED1 \\
\hline PDGFRB & SNX1 \\
\hline MYOD1 & TCF4 \\
\hline LCK & RASA1 \\
\hline CD74 & HLA \\
\hline BNIP2 & FGFR1 \\
\hline MDM2 & TP53 \\
\hline PDIA3 & SLC2A1 \\
\hline AVPR1A & PRKCA \\
\hline
\end{tabular}




\begin{tabular}{|c|c|}
\hline PDIA3 & SLC12A3 \\
\hline HSP90AA1 & PPID \\
\hline ARHGDIB & VAV1 \\
\hline SEMG1 & SEMG2 \\
\hline MCM6 & MLLT3 \\
\hline DLAT & PDK3 \\
\hline LMO2 & STAT1 \\
\hline DLG2 & GUCY1A2 \\
\hline BAT3 & PTPN12 \\
\hline IGF1 & IGFBP3 \\
\hline ARHGEF12 & ARHGEF12 \\
\hline GBP2 & HSPE1 \\
\hline FYN & MAP2 \\
\hline HMGN1 & HMGN1 \\
\hline MAPRE2 & MAPRE2 \\
\hline RAC1 & TIAM1 \\
\hline ATN1 & SIAH1 \\
\hline FYN & PTPN11 \\
\hline GTF3C1 & IGFBP3 \\
\hline CD2 & CD58 \\
\hline EP300 & KAT2B \\
\hline ATM & SMC1A \\
\hline FADD & HIPK3 \\
\hline AMBP & AMBP \\
\hline PTN & WFDC2 \\
\hline MCM2 & PHC2 \\
\hline S100A1 & S100P \\
\hline COX17 & EEF1A1 \\
\hline TNNC1 & TNNI1 \\
\hline MCM5 & NFKBIA \\
\hline ADRBK1 & PRKCB \\
\hline HDAC2 & PML \\
\hline SMAD3 & VIM \\
\hline DCTN1 & GRB2 \\
\hline CDKN1C & PCNA \\
\hline S100A9 & S100A9 \\
\hline CREBBP & NPAS2 \\
\hline NUCB1 & PTGS2 \\
\hline DLG2 & LRP2 \\
\hline CDKN2A & SLC4A1 \\
\hline CASP3 & CASP6 \\
\hline TNFSF9 & TRAF1 \\
\hline LCK & PECAM1 \\
\hline МАРЗК8 & RELA \\
\hline SNAP23 & STX3 \\
\hline JUN & NFE2L1 \\
\hline KITLG & KITLG \\
\hline
\end{tabular}




\begin{tabular}{|c|c|}
\hline GRB2 & JAK1 \\
\hline LRP8 & SNX17 \\
\hline МАР3К14 & TRAF3 \\
\hline ABL1 & JAK1 \\
\hline CD81 & ITGA4 \\
\hline $\mathrm{CHGB}$ & RXRG \\
\hline FGFR3 & HBZ \\
\hline ATF2 & SMAD3 \\
\hline EIF4A1 & EIF4G2 \\
\hline EIF2AK2 & ILF3 \\
\hline DAP3 & NR3C1 \\
\hline NCOA1 & PSMB9 \\
\hline CTTN & WASL \\
\hline DENND4A & YWHAB \\
\hline CRK & MAP4K5 \\
\hline HMGB1 & HNRNPK \\
\hline KAT5 & LRP1 \\
\hline CCNG1 & PPP2R4 \\
\hline STX4 & STXBP3 \\
\hline SKP2 & TAL1 \\
\hline POLR2G & POLR2G \\
\hline CDK8 & MED12 \\
\hline ANK3 & SMAD2 \\
\hline ATXN1 & CDK6 \\
\hline IL6ST & LIF \\
\hline TFAP2A & YBX1 \\
\hline KDR & NCK1 \\
\hline APLP1 & MPHOSPHE \\
\hline TFDP2 & YWHAE \\
\hline EEF1D & EEF1D \\
\hline E2F1 & SKP2 \\
\hline MAPK1 & PTPN7 \\
\hline CPB2 & F2 \\
\hline PLCG1 & SYK \\
\hline AP3B1 & ARF6 \\
\hline ITGAV & L1CAM \\
\hline BAT2 & IMMT \\
\hline AKT1 & МАР3К8 \\
\hline BDKRB2 & NOS1 \\
\hline ACY1 & ACY1 \\
\hline DAPK1 & FADD \\
\hline HSPG2 & PDGFA \\
\hline LRP1 & PDGFB \\
\hline $\mathrm{CDH} 1$ & CTNND1 \\
\hline CLTC & OCRL \\
\hline CCNG1 & MDM2 \\
\hline SEMA4D & SEMA4D \\
\hline
\end{tabular}




\begin{tabular}{|c|c|}
\hline PABPC1 & PCBP2 \\
\hline CRMP1 & FTH1 \\
\hline AHR & RELA \\
\hline BMP7 & ENG \\
\hline CDC6 & CDKN1A \\
\hline ACTG1 & PTPRO \\
\hline RAC1 & VAV1 \\
\hline DLG4 & HTR2A \\
\hline HDAC1 & NFKB1 \\
\hline $\mathrm{CDH} 2$ & CTNNB1 \\
\hline KIF5B & YWHAQ \\
\hline MYOD1 & SMAD4 \\
\hline PPP2CA & RPS6KB1 \\
\hline ClB1 & PSEN1 \\
\hline NFKBIA & PIK3R1 \\
\hline CDH5 & PTPN11 \\
\hline HLA & MAGEA4 \\
\hline CUL1 & E2F1 \\
\hline CBL & PTPN6 \\
\hline AHR & SMARCA4 \\
\hline CD36 & ITGB1 \\
\hline EGFR & ESR1 \\
\hline CBFB & CHGB \\
\hline LAPTM5 & USP13 \\
\hline IKBKE & KTN1 \\
\hline KRT10 & PRKCZ \\
\hline PIGA & $\mathrm{PIGH}$ \\
\hline HNF4A & NCOA1 \\
\hline EP300 & MYC \\
\hline PRLR & TEC \\
\hline PPARA & RXRA \\
\hline FUS & SFPQ \\
\hline NFKB2 & NKRF \\
\hline PTN & RPLP1 \\
\hline RAD51 & UBE2I \\
\hline TSC2 & YWHAE \\
\hline PIAS1 & PTPN1 \\
\hline DOK1 & KIT \\
\hline ETV6 & KAT5 \\
\hline HNRNPK & HNRNPK \\
\hline CDK9 & RB1 \\
\hline PIK3R1 & TGFBR1 \\
\hline FGF1 & FGFR2 \\
\hline PSME3 & TP53 \\
\hline ARNTL & HSP90AA1 \\
\hline NID2 & SKIL \\
\hline FANCA & FANCC \\
\hline
\end{tabular}




\begin{tabular}{|c|c|}
\hline UBE2I & UBE2I \\
\hline DDX11 & PFN2 \\
\hline DAXX & MAР3К5 \\
\hline MDFI & TRPV6 \\
\hline ARHGDIA & ARHGDIA \\
\hline PTK2B & RASA1 \\
\hline GNAI2 & GPSM2 \\
\hline CRKL & DOK1 \\
\hline ATN1 & MBP \\
\hline C19orf57 & VCL \\
\hline EP300 & NEUROD1 \\
\hline GRB2 & LCP2 \\
\hline ANXA5 & EIF4G1 \\
\hline SLC9A3R2 & SRY \\
\hline DLG4 & LRP8 \\
\hline EP300 & PROX1 \\
\hline TPD52 & TPD52 \\
\hline CTCF & YBX1 \\
\hline PDLIM1 & VIM \\
\hline GUCY1B3 & HSP90AA1 \\
\hline CREBBP & GATA1 \\
\hline PPP2CA & PPP2R5B \\
\hline ATXN1 & FYN \\
\hline TAF4 & TAF9 \\
\hline EP300 & RUNX3 \\
\hline PAK1 & PAK1 \\
\hline ATN1 & FBLN1 \\
\hline EGF & EGFR \\
\hline ICAM2 & RDX \\
\hline CDC20 & HDAC2 \\
\hline JUN & SMAD2 \\
\hline TRHR & TRHR \\
\hline BCL2 & TOMM20 \\
\hline NR3C1 & POU2F2 \\
\hline BLM & ТР53ВР1 \\
\hline МАР3К14 & RPL6 \\
\hline MAPK8IP2 & MAPK9 \\
\hline ACVRL1 & TGFB1 \\
\hline NCOA1 & VDR \\
\hline $\mathrm{NCL}$ & NPM1 \\
\hline $\mathrm{BCL} 3$ & NCOA1 \\
\hline FCN2 & MASP1 \\
\hline EP300 & IRF2 \\
\hline CCDC85B & NDUFA5 \\
\hline MYC & SMARCB1 \\
\hline NPPA & NPR3 \\
\hline ADRB3 & SRC \\
\hline
\end{tabular}




\begin{tabular}{|c|c|}
\hline CCNA1 & E2F1 \\
\hline DNTTIP2 & ESR1 \\
\hline HLA & LILRB1 \\
\hline ATF6 & ATF6B \\
\hline FHL2 & ITGA7 \\
\hline LPL & PTPN4 \\
\hline ARNTL & HIF1A \\
\hline HDAC2 & TP53 \\
\hline CCNA2 & E2F1 \\
\hline AMPH & BIN1 \\
\hline SLA & SYK \\
\hline ENO2 & HK1 \\
\hline GNAI2 & RGS4 \\
\hline CREBBP & MGMT \\
\hline ANG & ATP6AP1 \\
\hline CTSK & FGFR3 \\
\hline PRKAA1 & PRKAG1 \\
\hline PSEN1 & RAB11A \\
\hline MCM2 & MCM7 \\
\hline JUND & MEN1 \\
\hline$A R$ & CDK9 \\
\hline CSNK2A1 & PTEN \\
\hline PCBD1 & PCBD1 \\
\hline EP300 & SREBF2 \\
\hline IQGAP2 & RHOG \\
\hline TAF10 & TBP \\
\hline PLCG1 & WAS \\
\hline RELA & TAF1 \\
\hline EGFR & $\mathrm{SHC} 3$ \\
\hline GRB2 & WASL \\
\hline CETP & EWSR1 \\
\hline ABL1 & PIK3R1 \\
\hline EIF2S2 & EIF5 \\
\hline CREBBP & KLF4 \\
\hline MAPK8 & PIK3R1 \\
\hline GNAZ & RAP1GAP \\
\hline TAF1A & TAF1C \\
\hline CSF2 & SDC2 \\
\hline MAX & MYC \\
\hline RUNX1 & TLE1 \\
\hline CENPB & PARP1 \\
\hline AKAP12 & PRKAR2A \\
\hline ASS1 & ASS1 \\
\hline SQSTM1 & TRAF6 \\
\hline NKX2 & RARA \\
\hline MCM3 & MCM6 \\
\hline GRB2 & NPM1 \\
\hline
\end{tabular}




\begin{tabular}{|c|c|}
\hline DLG1 & KCNJ12 \\
\hline EIF4A2 & EIF4G2 \\
\hline HMGA1 & PPARG \\
\hline PTEN & UBE2L3 \\
\hline EP300 & NCOA6 \\
\hline POP1 & POP1 \\
\hline EGF & ERBB2 \\
\hline TAC1 & TACR1 \\
\hline DLG3 & LRP2 \\
\hline RAC1 & RAC1 \\
\hline EP300 & TCF4 \\
\hline BSG & SLC16A1 \\
\hline CFL1 & LIMK2 \\
\hline CPE & RPA1 \\
\hline DLG1 & GRIK2 \\
\hline ESR1 & SAFB \\
\hline ERBB4 & GRB2 \\
\hline NOS2 & RAC1 \\
\hline DAZ1 & PUM2 \\
\hline LYN & PIK3CG \\
\hline NDRG1 & PHYHIP \\
\hline PTPN1 & TRPV6 \\
\hline CBL & YWHAZ \\
\hline FASLG & LCK \\
\hline CREBBP & SMAD3 \\
\hline FOXG1 & SMAD1 \\
\hline CANX & CD1D \\
\hline CRABP1 & MPP3 \\
\hline TF & TFRC \\
\hline DAZAP2 & TLE1 \\
\hline CREB3 & MALL \\
\hline RASA1 & SYN1 \\
\hline MAP2K4 & MAP3K10 \\
\hline COPS6 & SMN1 \\
\hline FYN & KDR \\
\hline SFN & TNK1 \\
\hline SMAD1 & TTF1 \\
\hline CLCN3 & CLCN3 \\
\hline NAE1 & NEDD8 \\
\hline RPS6KA2 & RPS6KA2 \\
\hline CTNNB1 & SMAD3 \\
\hline ETV1 & КАT2B \\
\hline CCNA1 & RBL2 \\
\hline CD33 & PTPN6 \\
\hline ESR1 & NCOA6 \\
\hline DPYSL2 & NUMB \\
\hline BCL3 & FYN \\
\hline
\end{tabular}




\begin{tabular}{|c|c|}
\hline PTPN11 & SELE \\
\hline SPI1 & SPIB \\
\hline BCL2L1 & MCL1 \\
\hline BRAF & YWHAZ \\
\hline CPE & GTF3C1 \\
\hline COASY & KRT20 \\
\hline ERBB4 & SHC1 \\
\hline SH3BP2 & VAV1 \\
\hline ADRBK1 & MDM2 \\
\hline ANXA2 & PHB \\
\hline LRP8 & LRPAP1 \\
\hline AP2B1 & CLINT1 \\
\hline MYC & NFYB \\
\hline TRAF1 & TRAIP \\
\hline TNNC1 & TNNI3 \\
\hline RNF5 & UBE2D2 \\
\hline ERBB2 & JUP \\
\hline CSF3R & SHC1 \\
\hline NKRF & REL \\
\hline CSNK2A1 & HDAC1 \\
\hline $\mathrm{CDH} 5$ & SHC1 \\
\hline JAK3 & PRMT5 \\
\hline FGFR3 & GRB2 \\
\hline GHR & JAK1 \\
\hline ATN1 & KRT31 \\
\hline PGR & RELA \\
\hline GTF2I & SMAD2 \\
\hline GTF2F2 & MSX2 \\
\hline EED & ITGB7 \\
\hline CCDC85B & PKN1 \\
\hline COPS2 & THRA \\
\hline ESR2 & RBM39 \\
\hline SNRPB & SNRPD3 \\
\hline BRCA1 & CCND1 \\
\hline NCF4 & XRCC6 \\
\hline PMM1 & RAB6A \\
\hline РРP3СА & RCAN1 \\
\hline CSNK2A1 & PAFAH1B1 \\
\hline PARP1 & PRKDC \\
\hline NTF3 & NTRK2 \\
\hline CCDC106 & COPS6 \\
\hline EWSR1 & PLSCR1 \\
\hline GNB2L1 & IGF1R \\
\hline BCL2 & BIK \\
\hline BIN1 & SH3GL2 \\
\hline TGFB1 & YWHAE \\
\hline ESR1 & F0X01 \\
\hline
\end{tabular}




\begin{tabular}{|c|c|}
\hline MAPK1 & TH \\
\hline DLG3 & DLG4 \\
\hline ABL1 & GPX1 \\
\hline PLCG1 & SRC \\
\hline CCL20 & VCAN \\
\hline ADRBK1 & GIT2 \\
\hline BIN1 & BIN1 \\
\hline CBLB & PLCG2 \\
\hline CTSB & S100A10 \\
\hline BLMH & TRIO \\
\hline CAV1 & PDGFRA \\
\hline HNF1A & KAT2B \\
\hline GRK6 & SNCA \\
\hline HIPK3 & $Z Y X$ \\
\hline cox17 & PPBP \\
\hline SUMO1 & TDG \\
\hline PRDX1 & PRDX1 \\
\hline HBA1 & NAP1L1 \\
\hline ATF7 & TAF4 \\
\hline ARL4D & EIF2B1 \\
\hline CCL17 & VCAM1 \\
\hline PIN1 & RBPMS \\
\hline EPB41 & KPNA2 \\
\hline IL5RA & JAK1 \\
\hline PPP2CA & TLX1 \\
\hline RAD21 & SMC1A \\
\hline PRKCD & PRKCD \\
\hline PLK4 & PLK4 \\
\hline KRT15 & KRT18 \\
\hline ASAH1 & TSC22D1 \\
\hline RAD52 & WRN \\
\hline CASP8 & FADD \\
\hline CSNK2A2 & PTEN \\
\hline INSR & SMAD2 \\
\hline MADD & TNFRSF1A \\
\hline PRKCZ & YWHAB \\
\hline SERPINB9 & UNC119 \\
\hline PARP1 & POLA1 \\
\hline COPS6 & PBX2 \\
\hline FHL2 & ZBTB16 \\
\hline BAT2 & HNRNPA1 \\
\hline FKBP5 & IKBKE \\
\hline COIL & KPNA3 \\
\hline TAF1 & UBTF \\
\hline HSF2 & UBE2I \\
\hline EP300 & PPARA \\
\hline EPCAM & EPCAM \\
\hline
\end{tabular}




\begin{tabular}{|c|c|}
\hline BRCA1 & CDK4 \\
\hline PEX1 & PEX6 \\
\hline BCL2L1 & MAPK8 \\
\hline APP & CAV1 \\
\hline CCDC130 & ZNF165 \\
\hline CSTB & CTSB \\
\hline MVD & MVD \\
\hline ATXN1 & PEPD \\
\hline HCFC1 & ZBTB17 \\
\hline KLF5 & SET \\
\hline ATN1 & GAPDH \\
\hline CBL & FGR \\
\hline CRMP1 & RPA2 \\
\hline BAD & SNCA \\
\hline ATN1 & WWP2 \\
\hline GRB2 & USP8 \\
\hline ERBB2 & PAK1 \\
\hline ABL1 & SRC \\
\hline HLA & HLA \\
\hline TBP & TP53 \\
\hline TLE1 & TLE1 \\
\hline BRCA1 & BRCA1 \\
\hline ERBB2 & GRB2 \\
\hline $\mathrm{CFH}$ & CRP \\
\hline $\mathrm{CDH} 1$ & EZR \\
\hline EEF1A1 & HSPE1 \\
\hline FYN & TYK2 \\
\hline IL8 & IL8 \\
\hline AKT1 & $\mathrm{TSC} 2$ \\
\hline BRAF & YWHAB \\
\hline ATXN1 & VSNL1 \\
\hline RB1 & SNAPC1 \\
\hline PSMD11 & SMAD1 \\
\hline BLK & CBL \\
\hline CYFIP2 & FXR2 \\
\hline NR3C1 & PTMS \\
\hline GRIA1 & GRIK2 \\
\hline CBR1 & ERCC8 \\
\hline EP300 & TP73 \\
\hline E2F5 & RBL2 \\
\hline PSMF1 & RBMX \\
\hline CD44 & VCAN \\
\hline HDAC1 & SP1 \\
\hline EZR & TSC1 \\
\hline$A R$ & RAF1 \\
\hline FOS & HNF1A \\
\hline GRN & HSPG2 \\
\hline
\end{tabular}




\begin{tabular}{|c|c|}
\hline TSC2 & YWHAQ \\
\hline KLK3 & SERPINA1 \\
\hline MAPK1 & MKNK1 \\
\hline TSC1 & YWHAB \\
\hline HOXA9 & PLSCR1 \\
\hline NAP1L1 & NAP1L1 \\
\hline AKAP5 & PRKAR2A \\
\hline PSMD1 & TRAF6 \\
\hline COL4A1 & COL4A2 \\
\hline ERCC3 & TP53 \\
\hline CHRNA1 & ITGA7 \\
\hline ADAR & XPO1 \\
\hline CA9 & CTNNB1 \\
\hline RBL1 & SMAD4 \\
\hline CCNE1 & CDC25A \\
\hline CCNB1 & CDC25C \\
\hline CDK2 & PRKCH \\
\hline ATM & WRN \\
\hline RPS6KA1 & YWHAB \\
\hline NUP62 & NUTF2 \\
\hline GRIA4 & SDCBP \\
\hline CDKN1A & PARP1 \\
\hline AP2M1 & IKZF1 \\
\hline FASLG & FN1 \\
\hline PLCG2 & PRKD1 \\
\hline ITGAV & ITGB3 \\
\hline LRP1 & SERPINE1 \\
\hline TEAD4 & VGLL1 \\
\hline ABL1 & NCK1 \\
\hline FCGR2B & INPP5D \\
\hline BTK & GTF2I \\
\hline NEFL & PKN1 \\
\hline PAFAH1B1 & TUBA1A \\
\hline CTNND1 & ZBTB33 \\
\hline TNFSF10 & TNFSF10 \\
\hline NFKBIA & RPS6KA1 \\
\hline EEF2 & TP53 \\
\hline RPA1 & RPA2 \\
\hline IGF1R & PTPN1 \\
\hline STX1A & VIM \\
\hline MYBL2 & SKP2 \\
\hline AKT3 & PRKCZ \\
\hline IL6ST & LIFR \\
\hline HOXB7 & NFKBIA \\
\hline NCOR2 & NFKB1 \\
\hline ARL6IP1 & FXR2 \\
\hline CD7 & PIK3R1 \\
\hline
\end{tabular}




\begin{tabular}{|c|c|}
\hline ESR1 & MVP \\
\hline EXT2 & EXT2 \\
\hline CAPN2 & CAPNS1 \\
\hline IGF2 & NOV \\
\hline ACVR1 & SMAD5 \\
\hline МАР3К12 & RPL18A \\
\hline ATXN1 & RBPMS \\
\hline ILVBL & XRCC6 \\
\hline CBX3 & SP100 \\
\hline SP1 & TAF4 \\
\hline RPA2 & TLE1 \\
\hline SRC & TRAF3 \\
\hline ERBB4 & SNTB2 \\
\hline NR5A1 & SP1 \\
\hline MAP3K12 & RGS1 \\
\hline AK1 & FHL2 \\
\hline CDKN2A & E4F1 \\
\hline CDC25C & YWHAZ \\
\hline GNAI1 & RGS4 \\
\hline MAР3КЗ & MARK2 \\
\hline ATP2B4 & DLG3 \\
\hline ATP5C1 & PTN \\
\hline PRKCA & TIAM1 \\
\hline CDC25B & YWHAZ \\
\hline MYC & SMAD3 \\
\hline CCND1 & CDKN1A \\
\hline FOS & GTF2F2 \\
\hline TNFRSF1A & UBE2I \\
\hline DDX17 & NCOA2 \\
\hline B4GALNT1 & B4GALNT1 \\
\hline S100A13 & SYT1 \\
\hline SRP54 & SRP54 \\
\hline GRM3 & SDCBP \\
\hline NFKB1 & NFKBIB \\
\hline RIPK1 & TRAF3 \\
\hline $\mathrm{CCNH}$ & ERCC3 \\
\hline PTK2B & SLC2A1 \\
\hline BRCA1 & POLR2A \\
\hline IL1R1 & IL1RN \\
\hline MAPK7 & RAF1 \\
\hline PSMB1 & PSMB3 \\
\hline KLF6 & PNO1 \\
\hline PTK2B & VAV1 \\
\hline IRS1 & PIK3R3 \\
\hline EIF3E & IFIT1 \\
\hline AQP1 & MDFI \\
\hline PRDX6 & PRDX6 \\
\hline
\end{tabular}




\begin{tabular}{|c|c|}
\hline CSNK1A1 & RCC1 \\
\hline CCND1 & EP300 \\
\hline LRP2 & MAPK8IP2 \\
\hline C1D & TSNAX \\
\hline EGFR & PIK3R1 \\
\hline GATA2 & SPI1 \\
\hline DYNLL1 & NFKBIA \\
\hline KAT2B & NR4A1 \\
\hline NOS1 & PTPN6 \\
\hline JUP & TCF7L2 \\
\hline EGFR & INPPL1 \\
\hline ARHGEF12 & GNA13 \\
\hline CASP8 & FASLG \\
\hline CALR & VWF \\
\hline MED24 & SMAD1 \\
\hline MAPK1 & SHC1 \\
\hline DNMT1 & EED \\
\hline DNAJC7 & RAD9A \\
\hline OPRD1 & OPRM1 \\
\hline GRB2 & GRB2 \\
\hline APLP1 & TSC22D1 \\
\hline SFRS4 & SFRS6 \\
\hline CRY1 & PLSCR1 \\
\hline ITGB2 & RDX \\
\hline LYN & PTK2B \\
\hline RAD52 & RPA2 \\
\hline EED & PPP1R8 \\
\hline MAPK1 & TOB1 \\
\hline NXF1 & U2AF1 \\
\hline AKAP13 & RXRB \\
\hline FHL3 & FHL3 \\
\hline PLAU & SERPINB2 \\
\hline MCF2 & TNK2 \\
\hline DCC & SIAH1 \\
\hline POLR2B & POLR2H \\
\hline NDUFV2 & SP110 \\
\hline CDK5R1 & CHN1 \\
\hline RAD23A & XPC \\
\hline PTK2B & SYK \\
\hline CSNK2B & PRKCZ \\
\hline SYK & SYK \\
\hline FSHR & GRK6 \\
\hline SMAD1 & ZNF76 \\
\hline HSPA5 & LDLR \\
\hline HERPUD1 & RPA2 \\
\hline EPHB1 & GRB10 \\
\hline HOXD4 & PBX1 \\
\hline
\end{tabular}




\begin{tabular}{|c|c|}
\hline CALM1 & MY07A \\
\hline CCNA1 & RBM4 \\
\hline ANK3 & SCN2A \\
\hline ISCU & SFN \\
\hline MCL1 & PMAIP1 \\
\hline MYBL2 & PARP1 \\
\hline CDK9 & POLR2A \\
\hline PRKDC & WRN \\
\hline SELPLG & SELPLG \\
\hline GRB2 & SRC \\
\hline SELE & SELL \\
\hline KDR & SHC1 \\
\hline GADD45A & PCNA \\
\hline NROB1 & NRIP1 \\
\hline FTH1 & FTH1 \\
\hline FEZ1 & TXNDC9 \\
\hline FGFR1 & NCAM1 \\
\hline CHML & RAB6A \\
\hline MAPKAPK3 & TCF3 \\
\hline FN1 & VHL \\
\hline PSMD12 & TRAF6 \\
\hline BCL2L1 & HRK \\
\hline MDM2 & SUMO1 \\
\hline HBA1 & PTEN \\
\hline ITSN1 & SNAP23 \\
\hline PDGFB & THBS1 \\
\hline MYC & NMI \\
\hline GAB1 & PTPN11 \\
\hline CCL4 & CCL4 \\
\hline BRF1 & RB1 \\
\hline DNM1 & SH3GL2 \\
\hline ESR1 & GTF2H1 \\
\hline MAPK8 & SPIB \\
\hline ARL4D & SNRPN \\
\hline IFRD1 & RIT1 \\
\hline HSP90AB1 & YWHAB \\
\hline KDR & VEGFA \\
\hline MSN & NCF4 \\
\hline VASP & VASP \\
\hline EP300 & NAP1L1 \\
\hline FOXO1 & HNF4A \\
\hline CYTH2 & TRIM23 \\
\hline IRAK1 & SQSTM1 \\
\hline GRB2 & VAV1 \\
\hline MEF2C & SP1 \\
\hline CDK4 & MYOD1 \\
\hline COPS6 & EP300 \\
\hline
\end{tabular}




\begin{tabular}{|c|c|}
\hline MAPK3 & RPS6KA2 \\
\hline PRDX4 & PRDX4 \\
\hline CD8A & HLA \\
\hline ABL1 & EPHB2 \\
\hline GAPDH & SERPINB9 \\
\hline APEX1 & PCNA \\
\hline IGFBP5 & THBS1 \\
\hline KIT & KITLG \\
\hline HBEGF & ZBTB16 \\
\hline AHR & DAP3 \\
\hline CTNNB1 & ERBB2 \\
\hline SYMPK & WWOX \\
\hline GRB2 & SHB \\
\hline SCAMP1 & SCAMP1 \\
\hline ARHGDIG & RHOA \\
\hline GADD45A & MAР3К4 \\
\hline COIL & COIL \\
\hline BLM & CASP3 \\
\hline GSTO1 & SETDB1 \\
\hline IL4 & IL4R \\
\hline RARA & UBE3A \\
\hline SATB1 & UBE2I \\
\hline STAM & USP8 \\
\hline BRCA1 & TP53BP1 \\
\hline MBP & PRMT5 \\
\hline BAT3 & NOMO1 \\
\hline POLR2A & wwox \\
\hline MAGEA1 & TRIM31 \\
\hline TRAF6 & TRAF6 \\
\hline HNRNPK & RBM42 \\
\hline NR3C1 & PBX1 \\
\hline CALM1 & GLP1R \\
\hline AXIN1 & DAB2 \\
\hline LRP1 & MAPK8IP2 \\
\hline CHD4 & SMARCA4 \\
\hline PRKCQ & VAV1 \\
\hline SMAD2 & SMAD2 \\
\hline PPP2R1A & STRN3 \\
\hline KIAA0087 & TP53 \\
\hline ENO2 & TUBA4A \\
\hline PRKG1 & RGS2 \\
\hline NUP62 & NXF1 \\
\hline KCNJ11 & KCNJ8 \\
\hline MCM2 & RPA3 \\
\hline $\mathrm{CDH} 1$ & HDAC2 \\
\hline HMGB1 & UNC119 \\
\hline C3 & C5 \\
\hline
\end{tabular}




\begin{tabular}{|c|c|}
\hline EEF1A1 & SULT1E1 \\
\hline SRF & TEAD1 \\
\hline CBX1 & TRIM28 \\
\hline PIK3CD & PIK3R1 \\
\hline SFN & USP8 \\
\hline YWHAB & ZFP36 \\
\hline CHD8 & TOP3B \\
\hline NFKBIB & RXRA \\
\hline CUL1 & SMAD3 \\
\hline$A R$ & UBE2I \\
\hline TAF12 & TAF7 \\
\hline XRCC5 & XRCC6 \\
\hline KPNA2 & RECQL \\
\hline HK3 & IGFBP4 \\
\hline LYN & MUC1 \\
\hline GOLGB1 & SLC2A3 \\
\hline INSR & VAV1 \\
\hline ADRB2 & GRB2 \\
\hline EGFR & RASA1 \\
\hline CBL & ITSN1 \\
\hline CSK & HNRNPK \\
\hline MPHOSPH6 & UNC119 \\
\hline CRK & EPS15 \\
\hline CPB2 & PLG \\
\hline CTLA4 & LYN \\
\hline EPOR & SYK \\
\hline RB1 & TMPO \\
\hline LRP1 & LRPAP1 \\
\hline GRB2 & PDE6G \\
\hline FLAD1 & FLAD1 \\
\hline ARF5 & ARFIP2 \\
\hline AANAT & YWHAZ \\
\hline GPR183 & MTA1 \\
\hline LCK & PIK3R1 \\
\hline FLT4 & ITGB1 \\
\hline ATF4 & POLR2C \\
\hline DLG2 & DLG4 \\
\hline PF4 & THBD \\
\hline IGF1R & PIK3R3 \\
\hline FGG & FGG \\
\hline ATF7 & PTP4A1 \\
\hline SMAD2 & TGIF1 \\
\hline$A R$ & FOXA1 \\
\hline EEF1G & KARS \\
\hline DLG4 & ERBB2 \\
\hline PYGM & S100A1 \\
\hline CKS2 & CKS2 \\
\hline
\end{tabular}




\begin{tabular}{|c|c|}
\hline BRCA1 & BRCA2 \\
\hline ADAM15 & ARHGEF6 \\
\hline HNF4A & NR2C2 \\
\hline HERPUD1 & STARD9 \\
\hline NCOA6 & PRKDC \\
\hline RELA & RXRA \\
\hline MAPK10 & UNC119 \\
\hline SNRPE & SNRPE \\
\hline PSMD2 & PTN \\
\hline AIP & ARNT \\
\hline BMP7 & BMPR1A \\
\hline BARD1 & FEZ1 \\
\hline INSR & PRKCD \\
\hline F2 & FGA \\
\hline CYP2E1 & POR \\
\hline ETF1 & PPP2CA \\
\hline CRMP1 & NDUFV2 \\
\hline RPS27A & SMAD1 \\
\hline HMGA1 & POU3F1 \\
\hline CTNNB1 & FYN \\
\hline FGFR1 & FGFR1 \\
\hline FSCN1 & PRKCA \\
\hline SRI & SRI \\
\hline JAK1 & STAT5A \\
\hline NFKB1 & PPP4C \\
\hline CRYAA & CRYBB2 \\
\hline FANCA & TOP3A \\
\hline PSME1 & PSME1 \\
\hline VIM & YWHAZ \\
\hline CDC7 & ORC1L \\
\hline $\mathrm{KIT}$ & LCK \\
\hline GNB2L1 & PRKCB \\
\hline MCM4 & MCM7 \\
\hline NFIB & NFIC \\
\hline CD22 & SYK \\
\hline FOS & JUN \\
\hline FYN & TUBA3C \\
\hline CSRP3 & MYOG \\
\hline AR & HSP90AA] \\
\hline GRB2 & MAP4K1 \\
\hline RIT1 & RLF \\
\hline KPNA1 & LMO4 \\
\hline CREBBP & MLL \\
\hline TK1 & TLE1 \\
\hline ATP2B2 & DLG1 \\
\hline HK3 & ZBTB17 \\
\hline ETS2 & JUN \\
\hline
\end{tabular}




\begin{tabular}{|c|c|}
\hline PML & TGIF1 \\
\hline CRK & EPHA3 \\
\hline CSK & INSR \\
\hline CD8A & HLA \\
\hline PRMT5 & SNRPD1 \\
\hline HIF1A & MTA1 \\
\hline CANX & F8 \\
\hline GRN & HOXA1 \\
\hline MCM7 & ORC2L \\
\hline PRLR & YWHAZ \\
\hline LIF & LIFR \\
\hline NCOR2 & RBPJ \\
\hline GRB2 & IRS1 \\
\hline FTL & FTL \\
\hline DAZL & DAZL \\
\hline CITED1 & ESR1 \\
\hline EZR & ICAM1 \\
\hline AP2M1 & TGOLN2 \\
\hline GSK3B & NFKB1 \\
\hline EZH1 & ZMYND11 \\
\hline PIN1 & RBBP8 \\
\hline CCDC85B & TNNT1 \\
\hline CDK5R1 & CTNNB1 \\
\hline RB1 & SMARCA4 \\
\hline E2F1 & GTF2H1 \\
\hline ACTN2 & DLG4 \\
\hline KRAS & PIK3CG \\
\hline GRB2 & ITK \\
\hline CREBBP & ETS2 \\
\hline RBBP4 & RBBP7 \\
\hline RAD21 & STAG1 \\
\hline CD4 & IL16 \\
\hline MATR3 & PCBP1 \\
\hline GNB2L1 & IL2RB \\
\hline HLA & POMC \\
\hline CD44 & TGFBR1 \\
\hline FGF2 & FGFBP1 \\
\hline E2F1 & PURA \\
\hline CSF2RB & PTPN6 \\
\hline JUP & PTPRK \\
\hline ACTA2 & ССT5 \\
\hline ARHGEF1 & ARHGEF1 \\
\hline SRC & TYRO3 \\
\hline$A R$ & BAG1 \\
\hline FBL & SMN1 \\
\hline CSTF2 & CSTF3 \\
\hline FANCA & SMARCA4 \\
\hline
\end{tabular}




\begin{tabular}{|c|c|}
\hline PKP1 & VIM \\
\hline ANXA3 & UNC119 \\
\hline CBL & GRB2 \\
\hline CD81 & IFITM1 \\
\hline TNFRSF14 & TRAF5 \\
\hline ADCYAP1 & SCTR \\
\hline CSRP2 & PIAS1 \\
\hline DDX17 & HDAC1 \\
\hline ITSN2 & TBL3 \\
\hline COIL & PSMA1 \\
\hline TRIM21 & TRIM21 \\
\hline KHDRBS1 & PRMT1 \\
\hline CAV1 & TRPC1 \\
\hline NR4A1 & PML \\
\hline ERG & ETS2 \\
\hline $\mathrm{CCNH}$ & FUBP1 \\
\hline CIB1 & EXOSC10 \\
\hline ITGAM & PLAUR \\
\hline C3 & CFP \\
\hline RELA & TAF11 \\
\hline TKT & TKT \\
\hline LTA & TNFRSF1A \\
\hline PLCD1 & TGM2 \\
\hline BRCA1 & H2AFX \\
\hline MYC & TP73 \\
\hline BAD & YWHAH \\
\hline POLR2H & POLR2H \\
\hline NME1 & NME1 \\
\hline CDKN2A & UBE2A \\
\hline POU5F1 & SOX2 \\
\hline PKD1 & PKD2 \\
\hline PZP & TGFB1 \\
\hline GATA3 & TAL1 \\
\hline EEF1A1 & TAF9 \\
\hline CDC25B & MAPK14 \\
\hline NAPA & STX4 \\
\hline IRS1 & JAK1 \\
\hline NFKB1 & NFKB2 \\
\hline SKI & SKI \\
\hline JUN & NFYA \\
\hline CASP10 & CASP6 \\
\hline NMB & NMBR \\
\hline HCFC1 & SP1 \\
\hline BTK & VAV1 \\
\hline CDH5 & $\mathrm{P} 2 \mathrm{RX} 4$ \\
\hline ACTN2 & COIL \\
\hline ARL4A & KPNA2 \\
\hline
\end{tabular}




\begin{tabular}{|c|c|}
\hline MC4R & POMC \\
\hline ZBTB16 & ZBTB16 \\
\hline BCR & PTPN1 \\
\hline AKAP13 & PPARA \\
\hline NFKB1 & TP53BP1 \\
\hline ACTA1 & CCT5 \\
\hline DRAP1 & FEZ1 \\
\hline NONO & PIN1 \\
\hline HMGB1 & MECP2 \\
\hline F2 & ITGA2B \\
\hline MAPK1 & TNIP1 \\
\hline SETDB1 & TK1 \\
\hline WAS & WIPF1 \\
\hline NOS1 & VAC14 \\
\hline AXIN1 & DVL3 \\
\hline ELF1 & HMGA1 \\
\hline CREBBP & MYOD1 \\
\hline IL3 & IL3RA \\
\hline PPFIA1 & TNNT1 \\
\hline EP300 & HNRNPU \\
\hline SULT1E1 & SULT2B1 \\
\hline SREBF2 & SUMO1 \\
\hline ARL4D & UNC119 \\
\hline ERBB4 & NRG1 \\
\hline BAT3 & CSTF2 \\
\hline COPS6 & ERH \\
\hline KPNB1 & NUP153 \\
\hline KIT & PTPRO \\
\hline RXRA & THRA \\
\hline LIG1 & TUBB3 \\
\hline $\mathrm{CDH} 1$ & $\mathrm{VCL}$ \\
\hline IRS1 & PIK3CA \\
\hline ARVCF & $\mathrm{CDH} 15$ \\
\hline NRIP1 & RXRB \\
\hline ERBB2 & PLCG1 \\
\hline AR & SRC \\
\hline JUP & NFKBIE \\
\hline EEF1A1 & RPLP1 \\
\hline BRCA1 & SMC1A \\
\hline KCNJ12 & SNTA1 \\
\hline GRB2 & SH3BP2 \\
\hline BRCA2 & PLK1 \\
\hline PPARA & RXRG \\
\hline GABRA1 & PRKCG \\
\hline $\mathrm{ABCD} 2$ & PEX19 \\
\hline ARHGEF6 & PAK2 \\
\hline EP300 & GATA4 \\
\hline
\end{tabular}




\begin{tabular}{|c|c|}
\hline GNB1 & GNG4 \\
\hline EPHA2 & SHC1 \\
\hline DOK1 & INPP5D \\
\hline CHGB & POLR2E \\
\hline EGFR & STAT5B \\
\hline WRN & XRCC6 \\
\hline LYN & PDE4A \\
\hline BAT3 & IMMT \\
\hline PSMD11 & SMAD5 \\
\hline PHYHIP & SMARCC2 \\
\hline PRKCl & YWHAZ \\
\hline GNAI3 & S1PR1 \\
\hline PDAP1 & PDGFA \\
\hline BRCA1 & MDC1 \\
\hline LYN & PECAM1 \\
\hline HTATSF1 & POLR2A \\
\hline NCF2 & PRDX6 \\
\hline LRP2 & MAGI1 \\
\hline CUL2 & ZER1 \\
\hline ANXA6 & RASA1 \\
\hline ENG & TGFBR2 \\
\hline EIF2S2 & EIF4G2 \\
\hline PHB2 & PTMA \\
\hline ESR1 & SP1 \\
\hline LMNA & PRKCA \\
\hline GTF2B & IKZF1 \\
\hline NCOA2 & VDR \\
\hline FN1 & TGM2 \\
\hline KRT17 & KRT6A \\
\hline ADRM1 & PSMD4 \\
\hline PCNA & RPA1 \\
\hline ESR1 & MAPK1 \\
\hline IGF1R & RASA1 \\
\hline MATN1 & MATN2 \\
\hline FYN & HSP90AA1 \\
\hline ATF1 & CSNK2A2 \\
\hline DGKZ & SNTA1 \\
\hline ARHGDIG & RHOG \\
\hline VIM & XRCC4 \\
\hline E2F4 & TFDP1 \\
\hline ARAF & PIK3R1 \\
\hline $\mathrm{CCNH}$ & MTA1 \\
\hline AKAP1 & MYCBP \\
\hline DLG4 & GUCY1A2 \\
\hline CD24 & FGR \\
\hline IGF2 & IGFBP6 \\
\hline KLRC1 & KLRD1 \\
\hline
\end{tabular}




\begin{tabular}{|c|c|}
\hline DAZAP2 & DAZL \\
\hline PLD1 & RHOA \\
\hline BRCA1 & RELA \\
\hline SELE & SERPING1 \\
\hline KCNJ1 & SLC9A3R2 \\
\hline CA9 & CTNNA1 \\
\hline AKAP5 & GABRB3 \\
\hline ARNT & SIM2 \\
\hline CBFB & RUNX3 \\
\hline EIF2AK2 & MAP3K5 \\
\hline EZR & SLC9A3R2 \\
\hline PLCG1 & RET \\
\hline RELA & SP1 \\
\hline IQGAP2 & RELA \\
\hline HNRNPK & UBE2I \\
\hline CAPNS1 & RAB1A \\
\hline HRAS & TTC1 \\
\hline HDAC2 & PPARD \\
\hline JUP & MUC1 \\
\hline DRAP1 & TAF9 \\
\hline DR1 & DRAP1 \\
\hline CAV2 & RASA1 \\
\hline CTNNB1 & NFKB1 \\
\hline TAF12 & TAF5 \\
\hline DCLRE1A & TP53BP1 \\
\hline EHMT2 & KLF6 \\
\hline HDAC1 & PHB \\
\hline CRMP1 & HMGB1 \\
\hline C1QBP & MAPK3 \\
\hline ESR1 & NR2F6 \\
\hline BLK & CD79B \\
\hline CSNK2A2 & HSP90B1 \\
\hline HDAC1 & TXNIP \\
\hline JAK1 & RAF1 \\
\hline CRYAB & PSMA3 \\
\hline CDC16 & CDC27 \\
\hline IL12RB1 & IL23A \\
\hline EEF1A1 & SERPINB9 \\
\hline PTPRC & SEMA4D \\
\hline EXOSC10 & SCRIB \\
\hline DLG1 & KCNJ2 \\
\hline CRK & WEE1 \\
\hline NCOA2 & PPFIA1 \\
\hline BCL2L1 & CASP8 \\
\hline KAT2B & SATB1 \\
\hline C4A & CST3 \\
\hline GRB14 & INSR \\
\hline
\end{tabular}




\begin{tabular}{|c|c|}
\hline TBP & TEAD1 \\
\hline GUCY2F & GUCY2F \\
\hline RASA1 & ZAP70 \\
\hline POLR2A & SND1 \\
\hline CALM1 & RIT2 \\
\hline MDFI & MYOG \\
\hline GSK3B & MYC \\
\hline ABL1 & CRKL \\
\hline CREBBP & HDAC1 \\
\hline GAB1 & GRB2 \\
\hline PTPRF & TRIO \\
\hline CD44 & ERBB4 \\
\hline EEF1A1 & ZNF24 \\
\hline ADAM15 & SH3GL2 \\
\hline ANK1 & TTN \\
\hline DRD2 & FLNA \\
\hline ATRX & RAD51 \\
\hline POLA2 & SETDB1 \\
\hline CD74 & HLA \\
\hline DRD5 & GNA13 \\
\hline EGFR & EPS15 \\
\hline BARD1 & BCL3 \\
\hline$A R$ & SMAD4 \\
\hline ACVR2B & INHBB \\
\hline AR & MDM2 \\
\hline SCNN1A & WWP2 \\
\hline IGF2 & IGFBP1 \\
\hline FBLN1 & NOV \\
\hline LAMC1 & NID1 \\
\hline ARHGDIG & $\mathrm{RHOB}$ \\
\hline FAS & FAS \\
\hline THRB & THRB \\
\hline CKS1B & DUSP1 \\
\hline NTRK2 & PLCG1 \\
\hline PLSCR1 & SLC25A6 \\
\hline CCDC130 & ZBTB16 \\
\hline ERBB2 & MUC1 \\
\hline ATN1 & MAGI1 \\
\hline MYOG & SRF \\
\hline DMD & DTNA \\
\hline ARL4D & PRKCSH \\
\hline MCM7 & ORC1L \\
\hline NCOA1 & RARA \\
\hline RELA & RFC1 \\
\hline COPS2 & COPS6 \\
\hline ATN1 & LTBP1 \\
\hline LTF & LYZ \\
\hline
\end{tabular}




\begin{tabular}{|c|c|}
\hline KPNA2 & RELB \\
\hline ARID5A & ATXN1 \\
\hline AFAP1 & PRKCD \\
\hline PPP2R1A & SMAD2 \\
\hline FANCC & HSPA1A \\
\hline PTPN12 & SMAD5 \\
\hline PIK3R1 & PTPN6 \\
\hline ESR2 & MED1 \\
\hline MYBL2 & MYBL2 \\
\hline SMAD3 & ZBTB16 \\
\hline AES & ATN1 \\
\hline CSNK2B & LYN \\
\hline IL12RB1 & IL12RB2 \\
\hline CALCOCO2 & FASTK \\
\hline GTF2E1 & TCEA1 \\
\hline ABL1 & $\mathrm{ABL} 2$ \\
\hline CDKN1C & SKP2 \\
\hline CCDC85B & PSMC1 \\
\hline CSNK2A1 & FOS \\
\hline LIMK1 & YWHAZ \\
\hline COPS6 & SERPINA5 \\
\hline TCEB1 & VHL \\
\hline GCSH & ZBTB16 \\
\hline CREBBP & HTT \\
\hline SEC23A & SEC24C \\
\hline LIPC & LRP1 \\
\hline PIK3R1 & SHC1 \\
\hline $\mathrm{ACHE}$ & APP \\
\hline RXRA & SRC \\
\hline GFAP & PDLIM1 \\
\hline IGFBP5 & VTN \\
\hline MSX1 & MSX2 \\
\hline NFKB1 & TXN \\
\hline TRIM29 & TRIM29 \\
\hline EEA1 & EEA1 \\
\hline RAD23B & XPC \\
\hline ССТ3 & HNRNPAO \\
\hline FGA & $\mathrm{HRG}$ \\
\hline DHX9 & HNRNPC \\
\hline CSNK2A1 & XRCC4 \\
\hline CPSF6 & EWSR1 \\
\hline DOCK1 & RHOG \\
\hline AFAP1 & PRKCA \\
\hline TAF10 & TAF6 \\
\hline CALCOCO2 & SRI \\
\hline CBX3 & PIM1 \\
\hline ABLIM1 & CALCOCO2 \\
\hline
\end{tabular}




\begin{tabular}{|c|c|}
\hline$A R$ & GNB2L1 \\
\hline KAT5 & ZNF24 \\
\hline BRCA2 & SMAD3 \\
\hline GNA13 & TBXA2R \\
\hline CNR1 & GNAI3 \\
\hline DPF1 & RPN1 \\
\hline HMGB1 & TP73 \\
\hline ID2 & NEDD9 \\
\hline HNRNPK & HNRNPL \\
\hline FABP5 & S100A7 \\
\hline APEX1 & XRCC1 \\
\hline CRKL & ETV6 \\
\hline ADORA1 & P2RY1 \\
\hline MAPK1 & RAF1 \\
\hline IPO5 & NUP153 \\
\hline PAX6 & TBP \\
\hline DNM2 & PDE6G \\
\hline FYN & UNC119 \\
\hline CASP10 & CASP10 \\
\hline RAB3A & RABIF \\
\hline KAT2A & MYC \\
\hline CRMP1 & EIF2S2 \\
\hline TNFAIP3 & TNIP1 \\
\hline BAD & YWHAE \\
\hline CDC6 & MCM2 \\
\hline PAPPA & SMAD3 \\
\hline CDC6 & ORC2L \\
\hline PSMC1 & PSMD5 \\
\hline $\mathrm{AR}$ & GTF2F1 \\
\hline HSPA1A & SOX9 \\
\hline CDKN1A & PCNA \\
\hline PAICS & PAICS \\
\hline SNAP23 & STX4 \\
\hline KLRC3 & KLRD1 \\
\hline CDC20 & CDC27 \\
\hline MLLT10 & SS18 \\
\hline CACNA1C & CACNB3 \\
\hline CSNK2A1 & XK \\
\hline CREBBP & NUP98 \\
\hline CREM & TAF4 \\
\hline ANXA2 & MAP3K \\
\hline ARHGEF7 & SCRIB \\
\hline GTF2B & POLR2A \\
\hline CD226 & FYN \\
\hline AGAP2 & PIK3R1 \\
\hline C8B & CLU \\
\hline CCNA1 & MCM4 \\
\hline
\end{tabular}




\begin{tabular}{|c|c|}
\hline NCOA1 & NR2F6 \\
\hline FXR2 & NONO \\
\hline MED1 & RXRA \\
\hline BLM & RPA1 \\
\hline KIT & PTPN6 \\
\hline ESR1 & SMARCA4 \\
\hline CREB3 & HCFC1 \\
\hline EGFR & PTK2B \\
\hline SLC12A2 & SLC12A2 \\
\hline SREBF2 & SREBF2 \\
\hline BARD1 & SETDB1 \\
\hline CD19 & CD9 \\
\hline CAV1 & GJB2 \\
\hline КАТ2B & TP53 \\
\hline CTNNB1 & PTPRU \\
\hline FOS & RELA \\
\hline GNAQ & TTC1 \\
\hline MPP3 & RAB31 \\
\hline PSME1 & $\mathrm{VCL}$ \\
\hline TRPC1 & TRPC3 \\
\hline AKT1 & PRKCQ \\
\hline RALB & RALBP1 \\
\hline RXRA & TBP \\
\hline BARD1 & RBBP8 \\
\hline COIL & FXR2 \\
\hline MAPK14 & MAPKAPK2 \\
\hline CEBPD & RELA \\
\hline BLM & MLH1 \\
\hline NR3C1 & NR3C1 \\
\hline APBB1 & APLP1 \\
\hline SMAD4 & UBE2I \\
\hline EPO & EPOR \\
\hline DAXX & PML \\
\hline RB1 & TRAP1 \\
\hline BRCA1 & E2F4 \\
\hline KRT8 & MAPK14 \\
\hline MAF & sox9 \\
\hline HDAC1 & $\mathrm{NR} 2 \mathrm{~F} 2$ \\
\hline NR3C1 & SMARCA4 \\
\hline ILF3 & PRMT1 \\
\hline CRKL & EPOR \\
\hline SNAP23 & STX1A \\
\hline DRD4 & KCNJ9 \\
\hline PLD1 & PRKCA \\
\hline MARK3 & TCEA2 \\
\hline USP7 & ZMYND8 \\
\hline GLRX & МАР3К5 \\
\hline
\end{tabular}




\begin{tabular}{|c|c|}
\hline NUP62 & PBX2 \\
\hline DCTN1 & DST \\
\hline COIL & PRMT1 \\
\hline EP300 & STAT3 \\
\hline CAV1 & NGFR \\
\hline GNAQ & PTGIR \\
\hline CCND2 & CDK5 \\
\hline CCND1 & PCNA \\
\hline HDAC2 & SETDB1 \\
\hline NEK2 & PPP1R2 \\
\hline JUN & SKI \\
\hline NT5C2 & NT5C2 \\
\hline CSTF2 & IMMT \\
\hline PCBP1 & RALY \\
\hline FANCA & SPTAN1 \\
\hline EWSR1 & KCNMB1 \\
\hline DAXX & UBE2I \\
\hline STX5 & TP53 \\
\hline POLA1 & XRCC5 \\
\hline EIF3A & EIF3I \\
\hline APOA1 & GPLD1 \\
\hline SMAD1 & sox5 \\
\hline ARF1 & ARFIP2 \\
\hline EP300 & TAL1 \\
\hline MAP2K1 & MAP3K4 \\
\hline GLDC & GLDC \\
\hline FSCN1 & RAB1A \\
\hline SAT1 & TLE1 \\
\hline TGFB2 & VTN \\
\hline CCL14 & DDX39 \\
\hline BLM & WRN \\
\hline MAP3К5 & YWHAZ \\
\hline $\mathrm{CBL}$ & IGF1R \\
\hline INPP5D & PECAM1 \\
\hline ATXN3 & RAD23B \\
\hline BAT1 & EXOSC9 \\
\hline EEF1B2 & EEF1G \\
\hline GRIA4 & PRKCG \\
\hline DLX2 & MSX2 \\
\hline EZR & PRKCA \\
\hline $\mathrm{P} 4 \mathrm{HB}$ & PTN \\
\hline ARHGEF12 & PLXNB1 \\
\hline F10 & PLAT \\
\hline ERBB2 & PTK2B \\
\hline GRK5 & SNCB \\
\hline IGF1 & IGFBP2 \\
\hline EZR & L1CAM \\
\hline
\end{tabular}




\begin{tabular}{|c|c|}
\hline NCOA2 & THRB \\
\hline CDC5L & PPP1R8 \\
\hline LCP2 & SHC1 \\
\hline EIF1AX & EIF5 \\
\hline IMMT & TXNDC9 \\
\hline KPNA1 & STAT3 \\
\hline BRCA1 & STAT5A \\
\hline RIN2 & RIN2 \\
\hline CSNK1A1 & KPNA2 \\
\hline ESR1 & RELA \\
\hline CTNNA1 & JUP \\
\hline MAD1L1 & NONO \\
\hline COPS6 & HMOX2 \\
\hline PIAS1 & SP3 \\
\hline DMC1 & RAD51 \\
\hline ARF1 & CYTH2 \\
\hline CSNK1D & DVL3 \\
\hline PRKG1 & RAF1 \\
\hline FLNB & PSEN1 \\
\hline LMO2 & LYL1 \\
\hline PML & TOPBP1 \\
\hline DSG1 & PKP2 \\
\hline CREBBP & GTF2B \\
\hline GABPA & SP3 \\
\hline CFD & SERPINF2 \\
\hline IGF1R & NEDD4 \\
\hline МАРЗК11 & MAPK8IP2 \\
\hline MED21 & POLR2A \\
\hline ALOX12 & KRT5 \\
\hline CREB1 & EP300 \\
\hline ATN1 & EFEMP1 \\
\hline SMN1 & SNRPD2 \\
\hline LYN & MME \\
\hline CDKN1C & LIMK1 \\
\hline RNF2 & TFCP2 \\
\hline CENPA & PARP1 \\
\hline CTNNB1 & PIK3R1 \\
\hline CDKN1A & SET \\
\hline ESR2 & NCOA6 \\
\hline MMP1 & TIMP1 \\
\hline CNTF & CNTFR \\
\hline IGBP1 & PPP2CA \\
\hline CCL19 & CCR7 \\
\hline $\mathrm{HRC}$ & TRDN \\
\hline MAD1L1 & TRIM29 \\
\hline DAZAP2 & RBPMS \\
\hline ILF3 & PLSCR1 \\
\hline
\end{tabular}




\begin{tabular}{|c|c|}
\hline SLC6A4 & STX1A \\
\hline CREBBP & GLI3 \\
\hline SKIL & SNRNP70 \\
\hline GPS2 & TP53 \\
\hline CCDC85B & KRT18 \\
\hline APLP1 & NACA \\
\hline CSNK2A1 & FGF1 \\
\hline GDI2 & RAB9A \\
\hline ACVR2A & BMP7 \\
\hline FOS & RUNX2 \\
\hline MAPRE2 & MAPRE3 \\
\hline CREBBP & CSNK2A2 \\
\hline MAP3К5 & TXN \\
\hline MED21 & THRA \\
\hline PDCD11 & S100A8 \\
\hline PTPN2 & STAT1 \\
\hline BYSL & COIL \\
\hline HSPA5 & PSME3 \\
\hline FYN & PTK2B \\
\hline AKT1 & SMAD4 \\
\hline HTR1D & S1PR1 \\
\hline GRB2 & HTT \\
\hline MED24 & RXRA \\
\hline AKT1 & PAK1 \\
\hline CCDC85B & TNNI1 \\
\hline NR4A1 & TP53 \\
\hline KLC1 & SFN \\
\hline ССТ3 & XRCC6 \\
\hline GNAI1 & S1PR1 \\
\hline BYSL & TRO \\
\hline AKAP5 & PRKAR2B \\
\hline FLNA & FLNB \\
\hline ATOX1 & ATP7A \\
\hline PSMC5 & SP1 \\
\hline ARHGEF7 & CBLB \\
\hline RET & SHC1 \\
\hline CXCR4 & JAK3 \\
\hline EWSR1 & SF1 \\
\hline GABPA & HCFC1 \\
\hline JUN & TOP2A \\
\hline ATM & PEX5 \\
\hline PFN2 & TERF1 \\
\hline MCM5 & MDFI \\
\hline PSMC6 & PSMD9 \\
\hline E2F1 & TFDP2 \\
\hline BAT3 & TAC1 \\
\hline MCM4 & MCM4 \\
\hline
\end{tabular}




\begin{tabular}{|c|c|}
\hline ANXA5 & EED \\
\hline IGF1R & JAK1 \\
\hline DTNA & SNTA1 \\
\hline APOB & CANX \\
\hline SMAD1 & SNRNP70 \\
\hline CTNNB1 & TCF7L2 \\
\hline GNAQ & RGS2 \\
\hline PSMC3 & VHL \\
\hline NCK1 & NEDD9 \\
\hline HMGB1 & RELA \\
\hline U2AF1 & U2AF2 \\
\hline HPCA & NAIP \\
\hline IGF1R & IGF1R \\
\hline HIPK3 & LIMK2 \\
\hline EP300 & TRIP4 \\
\hline MAP3К5 & RAF1 \\
\hline BAT2 & CPSF1 \\
\hline CBX3 & MKI67 \\
\hline CD44 & NF2 \\
\hline CCND3 & CDKN1A \\
\hline LIG4 & XRCC4 \\
\hline ESR1 & TRIP4 \\
\hline MPZ & PMP22 \\
\hline PPP2R1B & PPP2R2A \\
\hline CDH5 & PKP4 \\
\hline RYR1 & RYR2 \\
\hline$A R$ & SMAD1 \\
\hline HIST1H2AC & TFAP2B \\
\hline ORC2L & ORC3L \\
\hline GRB2 & KRT7 \\
\hline BPTF & MAZ \\
\hline $\mathrm{BCR}$ & $\mathrm{HCK}$ \\
\hline RPL9 & RPS3 \\
\hline PEA15 & PLD1 \\
\hline BAT3 & CTSB \\
\hline FLT1 & KDR \\
\hline CRK & VAV1 \\
\hline ABL1 & ATM \\
\hline SF3B3 & TAF9 \\
\hline BCL2 & TMBIM6 \\
\hline HSPB1 & TP53 \\
\hline KIF5B & SNAP25 \\
\hline COPS6 & GPS1 \\
\hline SKIL & ZBTB6 \\
\hline GSK3B & MAPT \\
\hline GRB2 & RET \\
\hline FMR1 & FXR1 \\
\hline
\end{tabular}




\begin{tabular}{|c|c|}
\hline MAPK1IP1L & MAPK1IP1L \\
\hline TAF1A & TP53 \\
\hline CDKN1C & MYOD1 \\
\hline MITF & SUMO1 \\
\hline DLG4 & GRIK1 \\
\hline GNB2L1 & TYK2 \\
\hline FHL2 & SFPQ \\
\hline GLUL & SKIL \\
\hline PRKAA2 & PRKAG1 \\
\hline ATN1 & PFKL \\
\hline TNNT1 & TPM1 \\
\hline AKT2 & TCL1A \\
\hline PTK2B & PTPN6 \\
\hline ACTB & АCTB \\
\hline CCL2 & $\mathrm{CCL} 2$ \\
\hline SMN1 & SMN1 \\
\hline PRKCD & PTK2B \\
\hline CRK & RET \\
\hline DAXX & HDAC1 \\
\hline SCNN1A & STX1A \\
\hline EZH2 & RPN2 \\
\hline PSMB1 & PSMB5 \\
\hline NR3C1 & POU1F1 \\
\hline CRK & RAPGEF1 \\
\hline CCL5 & CCR1 \\
\hline FKBP3 & YY1 \\
\hline HNRNPD & SAFB \\
\hline DYNLT3 & DYNLT3 \\
\hline PAFAH1B2 & ZFP36L1 \\
\hline TERF2 & WRN \\
\hline ELK1 & MAPK8 \\
\hline NEDD4 & UBE2L3 \\
\hline MAP2K1 & MAPK3 \\
\hline ILF3 & MDFI \\
\hline MMP14 & TIMP2 \\
\hline KTN1 & RAC1 \\
\hline ARNT & EPAS1 \\
\hline B4GALT1 & LALBA \\
\hline BMI1 & IMMT \\
\hline CAPNS1 & CDK4 \\
\hline GTF2A2 & TAF4 \\
\hline NR3C1 & PSMC3IP \\
\hline MLF1 & YWHAZ \\
\hline CRMP1 & FXR1 \\
\hline HIF1A & VHL \\
\hline GNAI2 & RGS16 \\
\hline MAPK3 & RAF1 \\
\hline
\end{tabular}




\begin{tabular}{|c|c|}
\hline USP7 & WWP2 \\
\hline MRPL12 & MRPL12 \\
\hline ATRX & PTPN4 \\
\hline$A X L$ & GRB2 \\
\hline RFC5 & UNC119 \\
\hline CDK9 & IL6ST \\
\hline ACTA1 & CNN1 \\
\hline COL17A1 & CTNND1 \\
\hline BCL2L1 & IRS1 \\
\hline APPBP2 & PCSK5 \\
\hline NID1 & NID1 \\
\hline JUN & MAPK8 \\
\hline MCM3 & ORC2L \\
\hline GTF2B & NCOA4 \\
\hline IGF1 & IGFALS \\
\hline NR1H3 & RXRA \\
\hline DUSP6 & MAPK3 \\
\hline TPD52L1 & TPD52L1 \\
\hline IRF7 & TRAF6 \\
\hline APOE & LRP8 \\
\hline RRM1 & RRM2 \\
\hline PHC2 & PHC2 \\
\hline DUSP3 & MAPK3 \\
\hline KAT5 & SYN1 \\
\hline SND1 & STAT6 \\
\hline SQSTM1 & SQSTM1 \\
\hline CIB1 & PRKDC \\
\hline MEN1 & MLL \\
\hline HLA & TRA \\
\hline SP1 & SP4 \\
\hline PSMA1 & PSMA4 \\
\hline ATN1 & CRIP2 \\
\hline ASL & ASL \\
\hline KHDRBS1 & STAT3 \\
\hline F5 & F5 \\
\hline POLR2A & TCEA1 \\
\hline KHDRBS1 & NCK1 \\
\hline CBLB & CRKL \\
\hline AP1B1 & ATM \\
\hline CDC37 & CDK6 \\
\hline PLG & THBS1 \\
\hline PDK1 & PDK2 \\
\hline MYOD1 & RORA \\
\hline LEPR & PIN1 \\
\hline FYB & LCP2 \\
\hline HDAC2 & RBBP7 \\
\hline CREB1 & CREBBP \\
\hline
\end{tabular}




\begin{tabular}{|c|c|}
\hline ADAM15 & LYN \\
\hline MASP1 & MBL2 \\
\hline MAPK1 & PEA15 \\
\hline NCOA6 & RARA \\
\hline CCNE1 & SMARCC1 \\
\hline EPS15 & EPS15 \\
\hline BRCA1 & SMARCA2 \\
\hline HNRNPK & РСBP2 \\
\hline EIF3B & EIF3F \\
\hline PCNA & POLD2 \\
\hline MEP1A & PTH \\
\hline SERPINE1 & VTN \\
\hline APOA1 & PDE1A \\
\hline NFKBIE & SKP1 \\
\hline BST1 & BST1 \\
\hline PCNA & RFC1 \\
\hline TPM3 & TPM3 \\
\hline INPP1 & LIG1 \\
\hline TP53ВP2 & USP4 \\
\hline OPN1LW & RANBP2 \\
\hline ATXN1 & FDPS \\
\hline NFKBIA & NFKBIB \\
\hline CREM & SPI1 \\
\hline GTF2B & RELA \\
\hline IL10RA & IL10RB \\
\hline$A R$ & FLNA \\
\hline ATXN1 & PSMC3 \\
\hline РPP2CB & PPP2R1A \\
\hline APOB & SEC61B \\
\hline CNTNAP1 & RHOA \\
\hline RUNX1T1 & RUNX1T1 \\
\hline BRCA1 & DHX9 \\
\hline EEF1G & ILF2 \\
\hline NR3C1 & SFN \\
\hline ATN1 & RBM10 \\
\hline TP53 & UBE2A \\
\hline LCK & PTPRC \\
\hline EPR1 & F5 \\
\hline$A B \mid 2$ & PCM1 \\
\hline ITGB3BP & RXRA \\
\hline COPB2 & RGS4 \\
\hline SDC2 & SDCBP \\
\hline CREBBP & NFE2L2 \\
\hline ELK3 & TCF3 \\
\hline ITGA3 & RABIF \\
\hline KCNQ1 & TRAF6 \\
\hline MCM7 & SSRP1 \\
\hline
\end{tabular}




\begin{tabular}{|c|c|}
\hline СCT7 & EEF1G \\
\hline SETDB1 & SULT1E1 \\
\hline DAXX & PAX3 \\
\hline COPS6 & LPL \\
\hline PPP2R1B & PPP2R5D \\
\hline TAF10 & TAF5 \\
\hline PLCG1 & ZAP70 \\
\hline MAPK9 & PRKD1 \\
\hline МСM3 & MCM5 \\
\hline ENOX2 & MAGEA11 \\
\hline ARHGEF7 & $\mathrm{CBL}$ \\
\hline EDNRA & GNA11 \\
\hline RPLP2 & RPLP2 \\
\hline EEF1D & GARS \\
\hline CCND1 & ESR1 \\
\hline FKBP4 & HSP90AA1 \\
\hline COPS6 & LAMA4 \\
\hline NCOR2 & PPARA \\
\hline C5 & $\mathrm{C} 8 \mathrm{~B}$ \\
\hline PTPN2 & STAT3 \\
\hline CREBBP & NKX2 \\
\hline PKD1 & RGS7 \\
\hline ATF2 & SMAD4 \\
\hline HNRNPM & LYST \\
\hline PML & SUMO1 \\
\hline ATF2 & CSNK2A2 \\
\hline DHX9 & PRMT1 \\
\hline RPS14 & SMAD2 \\
\hline PSMA4 & PSMA5 \\
\hline CCNG2 & PPP2CA \\
\hline DAP3 & HIF1A \\
\hline STAT1 & TRADD \\
\hline FABP1 & PPARA \\
\hline GTF2B & GTF2F1 \\
\hline MEN1 & RBBP5 \\
\hline TAF6 & TAF6 \\
\hline CRK & ZAP70 \\
\hline CD46 & YES1 \\
\hline RELA & TWIST1 \\
\hline LYN & PRKDC \\
\hline NRCAM & PTPRB \\
\hline GHR & SHC1 \\
\hline SMG1 & UPF1 \\
\hline MAPK1 & RPS6KA2 \\
\hline CACNA1B & GNAO1 \\
\hline RB1 & RBBP7 \\
\hline OCLN & YES1 \\
\hline
\end{tabular}




\begin{tabular}{|c|c|}
\hline GNAQ & RGS16 \\
\hline NCSTN & PSEN2 \\
\hline CDK4 & CEBPA \\
\hline TAF10 & TAF11 \\
\hline BCL2 & SOD1 \\
\hline PRKAR1A & SMAD2 \\
\hline ATXN2L & EPOR \\
\hline GRIA1 & GRIA4 \\
\hline CASP10 & TNFRSF1A \\
\hline PCBP1 & PUF60 \\
\hline EIF2B1 & EIF2S1 \\
\hline PLCG2 & SHC1 \\
\hline ATXN1 & ZYX \\
\hline RBL1 & USP4 \\
\hline GABPA & SP1 \\
\hline APCS & C4BPA \\
\hline BTK & PLCG2 \\
\hline IGFBP5 & SERPINE1 \\
\hline CYTH1 & CYTIP \\
\hline МАРЗКЗ & YWHAZ \\
\hline MEN1 & NFKB1 \\
\hline CBL & SHC1 \\
\hline IL6ST & OSM \\
\hline EFNA5 & EPHB1 \\
\hline PML & RB1 \\
\hline CDKN1A & PSMA3 \\
\hline $\mathrm{ABL} 2$ & EPHB2 \\
\hline EWSR1 & NPPB \\
\hline CTNND1 & EGFR \\
\hline MATK & PXN \\
\hline F3 & FLNA \\
\hline PLSCR1 & SF1 \\
\hline PML & SRF \\
\hline FZD9 & MDFI \\
\hline COMMD1 & CUL2 \\
\hline EIF4A1 & EIF4G1 \\
\hline CSK & IGF1R \\
\hline ATF2 & UBE2I \\
\hline EWSR1 & SMAD4 \\
\hline EP300 & PCNA \\
\hline CD44 & EZR \\
\hline HDAC1 & PPARD \\
\hline ITSN1 & SCAMP1 \\
\hline GNAO1 & OPRD1 \\
\hline DHX9 & TOP2A \\
\hline GNAQ & VIPR1 \\
\hline LBR & SRPK1 \\
\hline
\end{tabular}




\begin{tabular}{|c|c|}
\hline GRB2 & SNTA1 \\
\hline FOS & MAPK1 \\
\hline EWSR1 & MYL6 \\
\hline KPNA1 & STAT1 \\
\hline PARP1 & TP53 \\
\hline AGT & EWSR1 \\
\hline PTK2B & PXN \\
\hline CRHR1 & GNAS \\
\hline SEPHS1 & UNC119 \\
\hline CHGA & PLG \\
\hline DGKZ & HRAS \\
\hline SETDB1 & TSC22D1 \\
\hline CD9 & PRKCA \\
\hline MARCKS & TOB1 \\
\hline ARNTL & CRY1 \\
\hline GSK3B & PTPN1 \\
\hline PIK3R1 & PIK3R1 \\
\hline CDKN2A & NPM1 \\
\hline STAT3 & STAT3 \\
\hline NMI & TUBA3C \\
\hline POLR2E & POLR2F \\
\hline GDNF & GFRA2 \\
\hline WAS & WIPF2 \\
\hline FGA & FGB \\
\hline IRF3 & IRF5 \\
\hline SKIL & UBE2I \\
\hline ATM & PRKDC \\
\hline CPSF1 & wWox \\
\hline TAF1 & TAF4 \\
\hline APPBP 2 & MLLT3 \\
\hline NEFH & NEFL \\
\hline GNAI3 & RGS3 \\
\hline MAP2 & MAP2 \\
\hline EIF2AK2 & EIF2AK2 \\
\hline EEF1A1 & PHYHIP \\
\hline GALT & TRIP13 \\
\hline PML & SMAD3 \\
\hline NEFL & NEFL \\
\hline DHFR & HSPD1 \\
\hline FASN & FASN \\
\hline EGFR & RGS16 \\
\hline VASP & WAS \\
\hline PLAT & SERPINE1 \\
\hline PCNA & RFC3 \\
\hline КАТ2B & SMAD3 \\
\hline CCR5 & CD4 \\
\hline DLG4 & PTK2B \\
\hline
\end{tabular}




\begin{tabular}{|c|c|}
\hline PTPRC & PTPRCAP \\
\hline CREBBP & STAT6 \\
\hline MAPK8 & TP53 \\
\hline ATP2A2 & PLN \\
\hline CD4 & KCNAB2 \\
\hline CDK7 & GTF2H1 \\
\hline SUMO2 & VIM \\
\hline RPS3A & SAP18 \\
\hline CITED1 & EP300 \\
\hline $\mathrm{FECH}$ & $\mathrm{FECH}$ \\
\hline ACTN2 & ATXN2 \\
\hline SKIL & SRP72 \\
\hline PF4 & PF4 \\
\hline $\mathrm{CDC7}$ & CHAF1A \\
\hline UQCRC1 & UQCRC2 \\
\hline CBL & YWHAQ \\
\hline DDR1 & RGS2 \\
\hline $\mathrm{A} 2 \mathrm{M}$ & AMBP \\
\hline NAPA & STX1A \\
\hline ID2 & MYF5 \\
\hline NR3C1 & STAT3 \\
\hline FGFBP1 & HSPG2 \\
\hline CD247 & LCK \\
\hline PSMB10 & PTN \\
\hline ARHGEF7 & PAK2 \\
\hline SCN5A & SNTA1 \\
\hline NCOA1 & NR5A1 \\
\hline NR3C1 & TXN \\
\hline PHC2 & RPL7 \\
\hline BAK1 & MCL1 \\
\hline GJA1 & MAPK7 \\
\hline CSNK2A1 & CSNK2B \\
\hline MAPK1 & SREBF1 \\
\hline AKT1 & NR4A1 \\
\hline MYB & PAX5 \\
\hline BAG1 & RAF1 \\
\hline MCM2 & SSRP1 \\
\hline GTF2E1 & TBP \\
\hline FEZ1 & TTR \\
\hline GADD45A & GADD45A \\
\hline TNFRSF1A & TRAF1 \\
\hline EP300 & KLF5 \\
\hline BTF3 & POLR2B \\
\hline EEF1A1 & PTPRCAP \\
\hline NFYB & NFYC \\
\hline CD22 & GRB2 \\
\hline CREBBP & CSNK2A1 \\
\hline
\end{tabular}




\begin{tabular}{|c|c|}
\hline BCL2L1 & PMAIP1 \\
\hline MYCBP & SSRP1 \\
\hline KRT20 & KRT20 \\
\hline DMD & SNTA1 \\
\hline NEDD4 & RPL18A \\
\hline KAL1 & SDC2 \\
\hline CANX & CD3D \\
\hline APLP1 & HLA \\
\hline KPNB1 & SMAD3 \\
\hline NR3C1 & NRIP1 \\
\hline COX6C & PTN \\
\hline PSMC1 & TRAF6 \\
\hline LGALS1 & PTPRC \\
\hline FAS & FYN \\
\hline CTNNB1 & EGFR \\
\hline IL6 & IL6R \\
\hline $\mathrm{BCL} 3$ & RXRA \\
\hline MCL1 & VDAC1 \\
\hline MYOD1 & MYOD1 \\
\hline MPRIP & SFN \\
\hline CSNK2A1 & EEF1D \\
\hline POLR2A & POLR2E \\
\hline FHL3 & ITGA7 \\
\hline CTSE & EMG1 \\
\hline EPHA2 & GRB2 \\
\hline BLM & TP53 \\
\hline RB1 & RB1 \\
\hline DSC2 & JUP \\
\hline PEX12 & PEX5 \\
\hline DHX34 & POLA2 \\
\hline C5AR1 & GNAI2 \\
\hline PTPN12 & STX16 \\
\hline PUF60 & U2AF2 \\
\hline CLIP1 & PAFAH1B1 \\
\hline SAT1 & SETDB1 \\
\hline HDAC2 & TOP2A \\
\hline NCOR2 & PGR \\
\hline ACVR2B & SNX1 \\
\hline APOB & HSP90B1 \\
\hline NF2 & PXN \\
\hline PSMA4 & PSMA4 \\
\hline JAK1 & TNFRSF1A \\
\hline SKP2 & TCF3 \\
\hline CD9 & ITGA5 \\
\hline BTK & HCK \\
\hline GRB2 & SKAP1 \\
\hline ABL1 & PLCG1 \\
\hline
\end{tabular}




\begin{tabular}{|c|c|}
\hline MAPK1IP1L & UBE2I \\
\hline HSPB3 & SETDB1 \\
\hline ESR1 & NCOA2 \\
\hline CDC6 & MCM7 \\
\hline CTNNB1 & TGFBR2 \\
\hline PBX1 & PKNOX1 \\
\hline CASP10 & CASP9 \\
\hline BIRC3 & CASP9 \\
\hline PSMC2 & TBP \\
\hline BLM & TOP3А \\
\hline SMN1 & TP53 \\
\hline GSTP1 & GSTP1 \\
\hline JUN & MAPK10 \\
\hline GRK5 & TACR1 \\
\hline HSF1 & HSPA1A \\
\hline BLM & MX1 \\
\hline PIP4K2B & PIP4K2B \\
\hline PCBP1 & SFRS3 \\
\hline EWSR1 & KRR1 \\
\hline MYC & ZBTB17 \\
\hline CDK2 & MCM4 \\
\hline DGKD & DGKD \\
\hline LCK & PIK3CA \\
\hline POLR2A & POLR2L \\
\hline DLST & $\mathrm{OGDH}$ \\
\hline SRC & WAS \\
\hline STX3 & STXBP2 \\
\hline DCTD & DCTD \\
\hline DNM1L & GSK3B \\
\hline PLK1 & PSMA4 \\
\hline HDAC1 & MTA1 \\
\hline FEZ1 & TOMM20 \\
\hline PPP2CA & PPP2R5C \\
\hline ADRBK1 & PDC \\
\hline GBP2 & SAT1 \\
\hline SNX17 & VLDLR \\
\hline EGFR & YWHAZ \\
\hline RALY & SPG7 \\
\hline IGFBP5 & PNO1 \\
\hline RPA1 & XPA \\
\hline CD34 & CRKL \\
\hline GTF2B & HSF1 \\
\hline EFNB3 & EPHB3 \\
\hline KLF5 & NFKB1 \\
\hline IGF1 & NOV \\
\hline TERT & YWHAQ \\
\hline ESR1 & POU4F2 \\
\hline
\end{tabular}




\begin{tabular}{|c|c|}
\hline PPFIA1 & PPFIA1 \\
\hline ACVR2B & INHBA \\
\hline SNRNP70 & SRPK1 \\
\hline ANK1 & TIAM1 \\
\hline DHX9 & HDLBP \\
\hline EP300 & TFAP2A \\
\hline BMP4 & BMPR1B \\
\hline HIPK3 & SIAH1 \\
\hline HDAC1 & TOP2B \\
\hline IGF1 & IGF1R \\
\hline SMAD3 & SMAD4 \\
\hline AHR & RB1 \\
\hline ADRB2 & OPRD1 \\
\hline CA2 & SLC9A1 \\
\hline PCNA & XRCC1 \\
\hline DAXX & MX1 \\
\hline GGA3 & M6PR \\
\hline MYLK & PAK1 \\
\hline ERCC5 & EWSR1 \\
\hline GPC1 & VEGFA \\
\hline DYNLL1 & MYO5A \\
\hline DLG4 & GRIK2 \\
\hline PPP1CC & TP53BP2 \\
\hline CRK & PLSCR1 \\
\hline EZR & SDC2 \\
\hline TP53 & VRK1 \\
\hline EP300 & NUP98 \\
\hline GFAP & MEN1 \\
\hline DLG3 & PTK2B \\
\hline FOS & SMAD3 \\
\hline JUP & PTPRF \\
\hline CASP7 & TNFRSF1A \\
\hline TPM1 & TPM2 \\
\hline $\mathrm{CBL}$ & FYN \\
\hline EGFR & SHC1 \\
\hline MORF4L2 & RB1 \\
\hline RAB4A & STX4 \\
\hline MX2 & MX2 \\
\hline PRKDC & XRCC4 \\
\hline PIM1 & SHMT1 \\
\hline RNF144A & UBE2L3 \\
\hline CPE & POLA2 \\
\hline MAP3K14 & MAP3К14 \\
\hline EMD & SH3GL3 \\
\hline SLC9A2 & SPTA1 \\
\hline MEP1A & MEP1A \\
\hline GSN & PXN \\
\hline
\end{tabular}




\begin{tabular}{|c|c|}
\hline RAF1 & YWHAZ \\
\hline HMGA1 & IRF1 \\
\hline ARHGDIA & RDX \\
\hline MYOD1 & TCF3 \\
\hline CRKL & PTPN11 \\
\hline EWSR1 & FASN \\
\hline $\mathrm{HCK}$ & WAS \\
\hline CD2 & CD48 \\
\hline ATP5C1 & PNO1 \\
\hline ARHGAP1 & RHOA \\
\hline CTNNB1 & SMAD4 \\
\hline RPA1 & SELENBP1 \\
\hline INSR & PTPN1 \\
\hline CCL3 & CCL4 \\
\hline ACVR2B & GDF5 \\
\hline TFAP2A & TP53 \\
\hline CD24 & LYN \\
\hline TLK1 & TLK1 \\
\hline CCL5 & CCR5 \\
\hline RAF1 & VDAC1 \\
\hline INPP5D & PTPN11 \\
\hline CCND1 & TAF1 \\
\hline GSK3B & TSC2 \\
\hline SMAD3 & SP1 \\
\hline ID3 & TCF3 \\
\hline TEP1 & TERT \\
\hline ITGB3 & PDGFRA \\
\hline CRK & PDGFRA \\
\hline CETN2 & XPC \\
\hline AKAP13 & THRA \\
\hline CALM1 & MYLK \\
\hline GANAB & PTPRC \\
\hline SMAD7 & SOX5 \\
\hline AFAP1 & AFAP1 \\
\hline HEXB & HEXB \\
\hline CNTNAP1 & FYN \\
\hline SFPQ & SFPQ \\
\hline ITSN2 & WAS \\
\hline JUN & SUMO2 \\
\hline HSPA1A & YWHAQ \\
\hline CRMP1 & TRIP13 \\
\hline HTR1A & S1PR1 \\
\hline PRKAA1 & PRKAB1 \\
\hline BAD & YWHAB \\
\hline KAT5 & MAD2L1BP \\
\hline PSG3 & SKIL \\
\hline PAX5 & TLE4 \\
\hline
\end{tabular}




\begin{tabular}{|c|c|}
\hline AKT1 & ILK \\
\hline LAMA3 & SDC2 \\
\hline EEF1A1 & STMN2 \\
\hline ERCC3 & GTF2H2 \\
\hline GNA13 & RGS16 \\
\hline $\mathrm{CHGB}$ & SAFB2 \\
\hline BRCA2 & TP53 \\
\hline CPSF6 & WWP2 \\
\hline PSMA3 & PSMA4 \\
\hline BCL2 & NR4A1 \\
\hline $\mathrm{DH} \times 9$ & SMN1 \\
\hline МАР3К12 & MAPK8IP2 \\
\hline EPRS & IARS \\
\hline BAD & EWSR1 \\
\hline CRIP1 & SH3GL3 \\
\hline IQGAP1 & TSG101 \\
\hline PLSCR1 & ZNF638 \\
\hline EPOR & GRAP \\
\hline RAF1 & YWHAE \\
\hline APOE & NEFM \\
\hline CTSB & RGS2 \\
\hline ARF6 & CYTH2 \\
\hline APP & GRB2 \\
\hline JAK1 & PRMT5 \\
\hline APOB & MTTP \\
\hline NXF1 & TNPO1 \\
\hline HIVEP1 & RAB1A \\
\hline CBL & UBE2L3 \\
\hline HOXC4 & XRCC6 \\
\hline MAX & SMAD4 \\
\hline PLCG2 & SYK \\
\hline CBL & NCK1 \\
\hline HIF1A & NR4A1 \\
\hline RUNX2 & SMAD3 \\
\hline GSTP1 & TGM2 \\
\hline COPS6 & CUL1 \\
\hline COPS5 & JUND \\
\hline CBX1 & CBX3 \\
\hline PKN2 & PLCG1 \\
\hline RELB & SMARCC2 \\
\hline DVL1 & SMAD3 \\
\hline ABL1 & ST5 \\
\hline APOA1 & APOA1 \\
\hline $\mathrm{DBH}$ & $\mathrm{DBH}$ \\
\hline MEF2A & SMAD2 \\
\hline NFKB2 & RELA \\
\hline GNAO1 & RGS19 \\
\hline
\end{tabular}




\begin{tabular}{|c|c|}
\hline RARA & ZBTB16 \\
\hline CAMK2G & SMAD4 \\
\hline LCK & SKAP1 \\
\hline ABCD1 & $A B C D 2$ \\
\hline TNF & TNFRSF1B \\
\hline MDM2 & TSG101 \\
\hline HNRNPC & UBE2I \\
\hline COPS2 & GFER \\
\hline CDC25B & YWHAQ \\
\hline ATN1 & PCSK5 \\
\hline CCND2 & PCGF2 \\
\hline RBBP4 & SP3 \\
\hline ENO1 & YWHAZ \\
\hline FLT3LG & FLT3LG \\
\hline PAFAH1B2 & PAFAH1B3 \\
\hline PRPS2 & PRPSAP1 \\
\hline ATXN1 & GSPT1 \\
\hline NDUFV3 & PHYHIP \\
\hline MED24 & PPARG \\
\hline CCNA2 & RBL2 \\
\hline TLE1 & TLE2 \\
\hline NFIB & RFX1 \\
\hline SMAD2 & TGM2 \\
\hline CBL & VAV1 \\
\hline CSNK2B & RPS6KB1 \\
\hline SLA & VAV1 \\
\hline USP8 & YWHAB \\
\hline NDN & NUCB2 \\
\hline PSMD8 & SMAD2 \\
\hline NQO2 & NQO2 \\
\hline MAPT & RPS6KB1 \\
\hline GRB2 & PTPN11 \\
\hline BTK & GNAQ \\
\hline EGFR & PLCG1 \\
\hline NFYC & SMAD2 \\
\hline ESR1 & RBM39 \\
\hline CRK & NCK1 \\
\hline BRCA1 & TP53 \\
\hline ATM & MRE11A \\
\hline DOK1 & RASA1 \\
\hline CLTC & IKBKE \\
\hline SNRPA & SNRPA \\
\hline CALCOCO2 & SMARCD1 \\
\hline CRK & SYN1 \\
\hline EPHA3 & TP53 \\
\hline FKBP1A & FKBP1A \\
\hline $\mathrm{CDH} 1$ & NEDD9 \\
\hline
\end{tabular}




\begin{tabular}{|c|c|}
\hline CD36 & ITGA2B \\
\hline FEZ1 & SMARCD1 \\
\hline LAMA4 & TP53 \\
\hline ANXA1 & S100A11 \\
\hline ITGB4 & SHC1 \\
\hline NFIC & ZNF167 \\
\hline GRK6 & RCVRN \\
\hline AR & STAT3 \\
\hline PAFAH1B3 & PAFAH1B3 \\
\hline CRP & SNRNP70 \\
\hline EFNA1 & EPHA4 \\
\hline GNAS & RGS2 \\
\hline CCNE1 & CUL3 \\
\hline DAP3 & HSP90AA1 \\
\hline NCOA6 & RB1 \\
\hline PKD2 & TNNI3 \\
\hline TNFRSF8 & TRAF5 \\
\hline CD247 & PTPRC \\
\hline BIRC2 & TRAF1 \\
\hline HLA & TAP2 \\
\hline POLR2A & POLR2G \\
\hline FEZ1 & HTT \\
\hline CDC37 & IKBKE \\
\hline ERBB3 & GRB7 \\
\hline CCNA1 & RBL1 \\
\hline ITPR3 & SIGMAR1 \\
\hline ACTG1 & CFL1 \\
\hline NEDD4 & UBE2D2 \\
\hline FGF2 & SDC1 \\
\hline MSX1 & MSX1 \\
\hline LAPTM5 & UBA52 \\
\hline HAPLN1 & VCAN \\
\hline PDLIM7 & ZNF165 \\
\hline CCND1 & RFC1 \\
\hline HNF4A & NCOA2 \\
\hline TNFAIP3 & YWHAE \\
\hline KRAS & RAF1 \\
\hline DDB1 & SKP2 \\
\hline BTK & BTK \\
\hline CREBBP & TP53 \\
\hline FASLG & MMP7 \\
\hline PSMD2 & PSMD5 \\
\hline EPOR & GRB2 \\
\hline KNG1 & PLAUR \\
\hline ITSN1 & LMO4 \\
\hline ITGB2 & KNG1 \\
\hline $\mathrm{CRH}$ & CRHR1 \\
\hline
\end{tabular}




\begin{tabular}{|c|c|}
\hline POLR2C & POLR2E \\
\hline AHR & ARNTL \\
\hline LAD1 & SFN \\
\hline PPP2R2A & TGFBR1 \\
\hline CD4 & CXCR4 \\
\hline ADRBK1 & EGFR \\
\hline DOCK1 & SRC \\
\hline MSX1 & PAX3 \\
\hline CRMP1 & VIM \\
\hline PTPRA & SRC \\
\hline JUN & MAPK9 \\
\hline CSNK2A1 & XRCC1 \\
\hline PTN & SAT1 \\
\hline APLP1 & TK1 \\
\hline SMAD2 & SRI \\
\hline SETDB1 & TOB1 \\
\hline HDAC1 & TPD52L1 \\
\hline ABL1 & TP53 \\
\hline PHYHIP & PRMT5 \\
\hline COIL & SART3 \\
\hline ERBB3 & IL6ST \\
\hline WWP1 & ZNF638 \\
\hline ACTN1 & MAGEA11 \\
\hline EXOC5 & PRKCG \\
\hline ESR1 & TP53 \\
\hline IKZF1 & UBE2I \\
\hline$A R$ & CCNE1 \\
\hline FHL3 & RBM42 \\
\hline RFC1 & RFC3 \\
\hline NCOR2 & RARA \\
\hline $\mathrm{CHM}$ & RAB3A \\
\hline FEZ1 & GTF2F1 \\
\hline EIF4E & EIF4E \\
\hline CCK & CCKBR \\
\hline TNFRSF1A & TNFRSF1A \\
\hline DOCK1 & RAC1 \\
\hline MYD88 & MYD88 \\
\hline MAGEA1 & SMCR7L \\
\hline OCRL & RAB1A \\
\hline FGF2 & FGFR1 \\
\hline MAP2 & MY07A \\
\hline MAPK7 & MEF2A \\
\hline DMD & SNTB1 \\
\hline GHR & STAT3 \\
\hline GSTP1 & PTN \\
\hline PSMC1 & PSMC2 \\
\hline BCL2 & BNIP2 \\
\hline
\end{tabular}




\begin{tabular}{|c|c|}
\hline CBLB & VAV1 \\
\hline BRCA1 & JUND \\
\hline MAP2K6 & МАР3К4 \\
\hline PAFAH1B3 & TLE1 \\
\hline PTPRK & TK1 \\
\hline BIRC3 & CASP7 \\
\hline NUP98 & TNPO1 \\
\hline HIF1A & NCOA1 \\
\hline $\mathrm{NCL}$ & PRKCZ \\
\hline ATXN1 & RCN1 \\
\hline EP300 & MDM4 \\
\hline PLN & SLN \\
\hline LMO3 & PHC2 \\
\hline EGFR & PIK3R2 \\
\hline EP300 & RORA \\
\hline PTN & SDC1 \\
\hline NONO & SPI1 \\
\hline RBPJ & SND1 \\
\hline EPS8 & EPS8 \\
\hline CXCL10 & CXCR3 \\
\hline CALCOCO2 & TCL1A \\
\hline ELF4 & RUNX1 \\
\hline EIF3F & HAX1 \\
\hline CSTF2 & SYMPK \\
\hline $\mathrm{CHGB}$ & MGLL \\
\hline SHC1 & STAT5B \\
\hline $\mathrm{BCR}$ & UNC119 \\
\hline GNAO1 & OPRM1 \\
\hline CD44 & FYN \\
\hline FAS & FASLG \\
\hline ATXN1 & ATXN2 \\
\hline BARD1 & XRCC6 \\
\hline AKT1 & RAF1 \\
\hline CBL & KDR \\
\hline COPS6 & CUL5 \\
\hline СТВР1 & NRIP1 \\
\hline FGFR3 & SLC25A6 \\
\hline TP53 & USP7 \\
\hline EP300 & SP1 \\
\hline ATN1 & PSMA3 \\
\hline INSR & RASA1 \\
\hline CDKN1A & GADD45A \\
\hline PTPRD & PTPRS \\
\hline CRKL & FCGR1A \\
\hline CDK5 & STX1A \\
\hline $\mathrm{E} 2 \mathrm{~F} 1$ & NDN \\
\hline MED1 & THRA \\
\hline
\end{tabular}




\begin{tabular}{|c|c|}
\hline TEAD1 & TEAD1 \\
\hline CDC25A & RAF1 \\
\hline PECAM1 & PIK3R1 \\
\hline MAPT & PIN1 \\
\hline PHYHIP & S100A13 \\
\hline CCL2 & DARC \\
\hline AKT1 & MAPKAPK2 \\
\hline MYOD1 & NR2F2 \\
\hline CASP9 & MAPK1 \\
\hline KPNA2 & RELA \\
\hline CCND2 & CDK4 \\
\hline COPS2 & THRB \\
\hline COL1A1 & MMP2 \\
\hline SSTR5 & SSTR5 \\
\hline BMP7 & BMPR2 \\
\hline PLK1 & PSMB7 \\
\hline ERBB4 & PTPN11 \\
\hline FN1 & MEP1A \\
\hline CHRNB1 & COPS6 \\
\hline HIF1A & OS9 \\
\hline SMARCA4 & STAT2 \\
\hline SMAD1 & SMAD5 \\
\hline DLST & ZBTB16 \\
\hline EWSR1 & SALL2 \\
\hline DNM2 & ITSN1 \\
\hline RFC4 & RFC5 \\
\hline CDK8 & POLR2A \\
\hline COPS6 & PSAP \\
\hline PRMT5 & PRMT5 \\
\hline ABL1 & GRB2 \\
\hline MAPK1 & VAV1 \\
\hline $\mathrm{CCNH}$ & GTF2H1 \\
\hline EGFR & PRKACA \\
\hline TAF10 & TAF9 \\
\hline BSG & MMP1 \\
\hline ACAN & TNFAIP6 \\
\hline MEOX1 & PAX3 \\
\hline CDK2 & MYBL2 \\
\hline C1R & C1S \\
\hline IRS1 & YWHAE \\
\hline ALG13 & ATN1 \\
\hline ACVR2A & GDF5 \\
\hline SLC9A2 & SRC \\
\hline HIF1A & MDM2 \\
\hline BLM & TERF2 \\
\hline APP & BLMH \\
\hline AKT1 & МАР3К11 \\
\hline
\end{tabular}




\begin{tabular}{|c|c|}
\hline ABL1 & MDM2 \\
\hline FHL2 & IGFBP5 \\
\hline NUP153 & XPO1 \\
\hline KAT5 & NDUFV2 \\
\hline MEF2C & NCOA2 \\
\hline TSC22D1 & TSC22D1 \\
\hline CD8A & PTPRC \\
\hline APPBP 2 & SYT11 \\
\hline CCDC85B & CDKN1A \\
\hline CSNK2A1 & SPIB \\
\hline MLH1 & PMS2 \\
\hline RER1 & SH3GL3 \\
\hline ELK1 & EP300 \\
\hline PIGR & RAB3B \\
\hline NROB2 & NR5A2 \\
\hline TAF13 & TBP \\
\hline EPOR & STAT5A \\
\hline MAPK8 & MYC \\
\hline EIF4A1 & EIF4G3 \\
\hline ARAF & RRAS \\
\hline TAF1B & TAF1C \\
\hline CASP3 & HSPD1 \\
\hline EIF4E & EIF4G2 \\
\hline TERT & XRCC6 \\
\hline SKIL & SMAD3 \\
\hline FUBP1 & VIM \\
\hline ATRX & PTN \\
\hline PGM1 & S100A1 \\
\hline COPS6 & SULT1E1 \\
\hline CDR2 & PKN1 \\
\hline FANCC & HSP90B1 \\
\hline KRT18 & KRT8 \\
\hline ELF1 & REL \\
\hline DYNLL1 & TP53BP1 \\
\hline IFT88 & RPGR \\
\hline FYN & THY1 \\
\hline ATF6 & YY1 \\
\hline CDK7 & ESR1 \\
\hline$A R$ & HMGB1 \\
\hline PLAG1 & SMAD3 \\
\hline ARPC1B & WAS \\
\hline $\mathrm{F} 2$ & THBS1 \\
\hline NCOA6 & XRCC6 \\
\hline NFKBIB & SKP1 \\
\hline CTNNB1 & PTPRZ1 \\
\hline SF1 & U2AF2 \\
\hline APC & PPP2R5A \\
\hline
\end{tabular}




\begin{tabular}{|c|c|}
\hline PAPOLA & REL \\
\hline EIF3F & PTN \\
\hline $\mathrm{CDH} 1$ & EGFR \\
\hline PDGFRA & PDGFRA \\
\hline HSP90AA1 & RIPK1 \\
\hline ARSE & TNK2 \\
\hline CRYBB1 & CRYBB1 \\
\hline ARL3 & GOLGA4 \\
\hline TRIM32 & TRIM32 \\
\hline GRM1 & GRM1 \\
\hline NTS & NTSR1 \\
\hline GAB1 & PIK3R1 \\
\hline LDLR & SNX17 \\
\hline TAF9 & TP53 \\
\hline CEBPE & MYB \\
\hline ARHGDIA & RAC2 \\
\hline CSK & PXN \\
\hline BGN & MFAP2 \\
\hline HRAS & SRC \\
\hline CYFIP2 & FXR1 \\
\hline IFNGR1 & JAK1 \\
\hline PRKCA & YWHAZ \\
\hline FYN & ZAP70 \\
\hline ACTB & ССТ2 \\
\hline ACTN1 & XRCC4 \\
\hline IL6R & IL6ST \\
\hline CD44 & TIAM1 \\
\hline CD2 & FYN \\
\hline FEZ1 & HTATSF1 \\
\hline SNCA & SNCB \\
\hline NUP62 & XPO1 \\
\hline ARHGAP1 & SRC \\
\hline NRIP1 & PPARA \\
\hline EZR & SPN \\
\hline RBBP4 & RPN1 \\
\hline ERCC2 & GTF2H1 \\
\hline PDGFRB & PDGFRB \\
\hline NEFL & VIM \\
\hline AMPH & DNM2 \\
\hline CLTC & TNK2 \\
\hline SETDB1 & SLC38A3 \\
\hline MAP3K5 & QARS \\
\hline MAPK3 & PEA15 \\
\hline EIF3A & EIF4G2 \\
\hline EPHA4 & FYN \\
\hline IL9R & YWHAZ \\
\hline$A R$ & $\mathrm{CDC} 25 \mathrm{~B}$ \\
\hline
\end{tabular}




\begin{tabular}{|c|c|}
\hline MTA1 & TP53 \\
\hline BPTF & H3F3A \\
\hline PDGFRB & PTPN11 \\
\hline PRMT1 & PRMT1 \\
\hline GRK5 & SNCA \\
\hline IL1B & IL1R1 \\
\hline ZNF174 & ZNF174 \\
\hline GTF2I & MAPK3 \\
\hline RAD51 & RPA1 \\
\hline EGFR & PTPN6 \\
\hline TOMM20 & UCP1 \\
\hline CA8 & ITPR1 \\
\hline SNX1 & SNX1 \\
\hline MAР3К4 & RAC1 \\
\hline CSNK2A2 & FGF1 \\
\hline COL1A1 & IGFBP3 \\
\hline CEACAM1 & PXN \\
\hline ATXN1 & ITGB4 \\
\hline BCAP31 & BCAP31 \\
\hline CSNK2A2 & PIN1 \\
\hline BNIP2 & BNIP2 \\
\hline TG & TG \\
\hline BAD & SFN \\
\hline FCGR3B & IGHG1 \\
\hline NR1I3 & POU1F1 \\
\hline ERCC2 & ERCC5 \\
\hline ICAM1 & IL2RA \\
\hline DDX17 & SNRNP70 \\
\hline SCNN1A & USP10 \\
\hline MDC1 & TP53BP1 \\
\hline RBBP8 & RBL2 \\
\hline CREBBP & ESR1 \\
\hline EIF2B2 & EIF2B5 \\
\hline ESR1 & PRMT1 \\
\hline APC & GSK3B \\
\hline NFKB1 & STAT3 \\
\hline FYN & LCP2 \\
\hline ANXA11 & ENO2 \\
\hline COPS6 & PTEN \\
\hline SORD & SORD \\
\hline MEF2C & TEAD1 \\
\hline ATF2 & HMGA1 \\
\hline GTF2I & NFKB2 \\
\hline GAS6 & MERTK \\
\hline KPNB1 & TP53 \\
\hline LRP1 & SNX17 \\
\hline ALDH1A1 & ALDH1A1 \\
\hline
\end{tabular}




\begin{tabular}{|c|c|}
\hline CRMP1 & HSPE1 \\
\hline CDK2 & ORC2L \\
\hline IRF9 & STAT2 \\
\hline SMARCB1 & SMARCB1 \\
\hline CRK & PSMC6 \\
\hline PTEN & UBE2I \\
\hline FIG4 & VAC14 \\
\hline JUN & SMAD3 \\
\hline ESR2 & MAD2L1 \\
\hline COPS5 & SMAD2 \\
\hline FGA & PLAT \\
\hline RNF10 & TLE1 \\
\hline MPP3 & RPS2 \\
\hline KPNB1 & RUNX1T1 \\
\hline ITK & ITK \\
\hline KIF5B & SFN \\
\hline ABAT & ALDH5A1 \\
\hline NUP98 & TPR \\
\hline RPL18A & TNK2 \\
\hline DVL3 & ZBTB48 \\
\hline NCOR2 & NFKBIA \\
\hline DAXX & SUMO1 \\
\hline HNRNPK & PRMT1 \\
\hline LRPAP1 & VLDLR \\
\hline CNR1 & GNAI1 \\
\hline CHRNA4 & YWHAH \\
\hline PHB & RBL2 \\
\hline PLK1 & PSMB3 \\
\hline TAF11 & TBP \\
\hline FYN & HRAS \\
\hline CASP8 & CASP8 \\
\hline PIK3R1 & RAC1 \\
\hline FHL3 & PHC2 \\
\hline ADRB2 & SLC9A3R2 \\
\hline LTBR & LTBR \\
\hline CD63 & HLA \\
\hline CITED2 & CREBBP \\
\hline KHDRBS1 & LYN \\
\hline IQGAP1 & PRKACA \\
\hline DRAP1 & NFYB \\
\hline INSR & SNX1 \\
\hline CDK2 & RBL2 \\
\hline MITF & PAX6 \\
\hline CCND3 & CRABP2 \\
\hline RAE1 & SFN \\
\hline EEF1A1 & PLCG1 \\
\hline TAF10 & TAF7 \\
\hline
\end{tabular}




\begin{tabular}{|c|c|}
\hline BIRC2 & CASP7 \\
\hline CD3E & CD3G \\
\hline SST & SSTR3 \\
\hline CD14 & LTF \\
\hline CTNNA1 & TJP2 \\
\hline SAT1 & SEPHS1 \\
\hline STAT1 & STAT2 \\
\hline NXF1 & RAE1 \\
\hline SMAD5 & TOB1 \\
\hline ACTN1 & SRC \\
\hline ACVR1 & SMAD1 \\
\hline HDAC2 & RCOR1 \\
\hline COPS6 & PFKL \\
\hline $\mathrm{MSH} 2$ & MSH6 \\
\hline ANP32A & ATXN1 \\
\hline ACTB & DSTN \\
\hline ATF1 & HNF1B \\
\hline PDGFA & PDGFRA \\
\hline CREB1 & VIM \\
\hline IL4R & INPP5D \\
\hline MY05A & RAB27A \\
\hline GTF2B & REL \\
\hline ATN1 & NELL1 \\
\hline CYC1 & CYCS \\
\hline CDC16 & SMAD3 \\
\hline TNFAIP3 & YWHAB \\
\hline IL4R & PTPN11 \\
\hline ADRBK1 & CCR4 \\
\hline ATP5C1 & CCND3 \\
\hline NFIX & PIR \\
\hline DCTN2 & GNAO1 \\
\hline EWSR1 & WWP2 \\
\hline CRK & SHB \\
\hline CREBBP & SREBF1 \\
\hline MEN1 & RPA2 \\
\hline GRN & TOP3B \\
\hline EMG1 & KHDRBS1 \\
\hline PRKCE & TIAM1 \\
\hline EIF2S2 & TP53 \\
\hline APOC2 & LPL \\
\hline CD2 & PTPRC \\
\hline MAРК9 & TP53 \\
\hline CYFIP2 & WASF1 \\
\hline HNF1A & HNF1A \\
\hline CTNNB1 & PIN1 \\
\hline HDAC1 & ZBTB16 \\
\hline USP8 & YWHAZ \\
\hline
\end{tabular}




\begin{tabular}{|c|c|}
\hline AP2M1 & CTLA4 \\
\hline DFFA & YWHAZ \\
\hline CAPN2 & NMT1 \\
\hline FOXO4 & SMAD4 \\
\hline CTLA4 & LCK \\
\hline COMMD1 & RELA \\
\hline CD55 & CD55 \\
\hline FEZ1 & TLK2 \\
\hline FYN & RAF1 \\
\hline HMGN1 & YWHAZ \\
\hline RFX5 & RFXAP \\
\hline EGF & EGF \\
\hline JAK1 & TSHR \\
\hline BLM & RAD51 \\
\hline S100B & TP53 \\
\hline DLG2 & KCNA4 \\
\hline ARHGEF1 & GNA13 \\
\hline CD44 & LCK \\
\hline CEBPA & NR3C1 \\
\hline MAP2K3 & MAP3K \\
\hline VEGFA & VTN \\
\hline CCR5 & STAT3 \\
\hline ACP1 & CTNNB1 \\
\hline ESR1 & PTPN6 \\
\hline МАРЗКЗ & MAP3КЗ \\
\hline MED12 & sox9 \\
\hline KRT15 & KRT6A \\
\hline ILK & LIMS1 \\
\hline ELK1 & MAPK1 \\
\hline $\mathrm{A} 2 \mathrm{M}$ & LEP \\
\hline HOXA1 & PLSCR1 \\
\hline $\mathrm{CDC} 27$ & PPP5C \\
\hline CCL3 & CCR4 \\
\hline BCAP31 & BCL2L1 \\
\hline EIF4G2 & MKNK1 \\
\hline$A R$ & TGIF1 \\
\hline L1CAM & PEA15 \\
\hline HMGA1 & JUN \\
\hline BCL2L1 & CYCS \\
\hline CASP2 & CCND3 \\
\hline ENPP1 & INSR \\
\hline DYNLL1 & EEF1A1 \\
\hline GNA13 & PRKCB \\
\hline MAD2L1BP & TP53 \\
\hline COPS5 & GPS1 \\
\hline GRIN1 & GRIN2B \\
\hline MYC & YY1 \\
\hline
\end{tabular}




\begin{tabular}{|c|c|}
\hline CD79A & SYK \\
\hline GSR & GSR \\
\hline CDK7 & GTF2H2 \\
\hline АСТВ & SMARCA4 \\
\hline GNB2L1 & PTPRM \\
\hline MCM3 & MCM4 \\
\hline IL2RG & SHC1 \\
\hline DDX18 & MTA1 \\
\hline IL10 & IL10 \\
\hline CDH5 & JUP \\
\hline ARF1 & ARFIP1 \\
\hline IL1R1 & MYD88 \\
\hline MAPK3 & SREBF1 \\
\hline ITGB1BP1 & LRP1 \\
\hline PIAS1 & SMAD4 \\
\hline BRCA2 & SHFM1 \\
\hline GAD1 & GAD2 \\
\hline CCND1 & THRB \\
\hline KIFAP3 & MAP3K10 \\
\hline RELA & TERT \\
\hline CIB1 & PLK3 \\
\hline GRB2 & SHC1 \\
\hline CAV1 & RCVRN \\
\hline CSNK2B & FGF1 \\
\hline HNRNPC & LMO3 \\
\hline TNFAIP3 & YWHAZ \\
\hline COL17A1 & ITGB4 \\
\hline BAG1 & ESR1 \\
\hline EGFR & HBEGF \\
\hline MAP2K6 & PLCB2 \\
\hline MAX & MYCN \\
\hline CD74 & HLA \\
\hline $\mathrm{BCL} 2$ & PPP2R5A \\
\hline SMAD3 & TOB1 \\
\hline PIK3R4 & YWHAB \\
\hline EPHB2 & EPHB2 \\
\hline PPP2CA & TRIP13 \\
\hline ATM & CREB1 \\
\hline NFKBIA & RELB \\
\hline LIMS1 & RBBP8 \\
\hline CD4 & LGALS1 \\
\hline SRPK2 & U2AF2 \\
\hline CXADR & EWSR1 \\
\hline BIRC3 & CASP3 \\
\hline ASCL1 & MEF2A \\
\hline TP53 & WRN \\
\hline DPH1 & HSPA5 \\
\hline
\end{tabular}




\begin{tabular}{|c|c|}
\hline ITGB3 & PECAM1 \\
\hline FXR2 & HNRNPC \\
\hline $\mathrm{CDH} 3$ & CTNNB1 \\
\hline FOXO1 & YWHAZ \\
\hline DDR2 & SHC1 \\
\hline DAXX & FAS \\
\hline CDH5 & VIM \\
\hline PPARD & PPARD \\
\hline HMGB1 & PLG \\
\hline АCTA1 & SPTAN1 \\
\hline GSTP1 & MAPK8 \\
\hline SMAD3 & TFE3 \\
\hline DSG1 & PKP1 \\
\hline PUM2 & PUM2 \\
\hline B2M & HLA \\
\hline COPS6 & RFC5 \\
\hline TRADD & TRADD \\
\hline C3 & CPN1 \\
\hline CTNNB1 & FOXO4 \\
\hline AKT1 & PKN2 \\
\hline ARAF & TIMM44 \\
\hline CCND2 & CDKN1A \\
\hline NTRK3 & PTPN1 \\
\hline GRB2 & RASA1 \\
\hline $\mathrm{ABI} 2$ & SNAP23 \\
\hline CD48 & EEF1D \\
\hline BCAP31 & CASP8 \\
\hline PSPH & $\mathrm{PSPH}$ \\
\hline HSPB2 & HSPB3 \\
\hline CALM1 & EWSR1 \\
\hline CDK2 & POLA1 \\
\hline IMMT & IMMT \\
\hline ATP5B & PSME3 \\
\hline ADRA2C & EIF2B1 \\
\hline AKT1 & YWHAZ \\
\hline ADRBK1 & FSHR \\
\hline MNT & MNT \\
\hline NUP153 & NXF1 \\
\hline ATF2 & RB1 \\
\hline POR & XRCC6 \\
\hline ARF1 & EEF1G \\
\hline RPS3A & SOD2 \\
\hline LYN & NEDD9 \\
\hline RIPK1 & TAX1BP1 \\
\hline C3 & ITGAX \\
\hline $\mathrm{CHGB}$ & SLC25A6 \\
\hline LAMC1 & NID2 \\
\hline
\end{tabular}




\begin{tabular}{|c|c|}
\hline TNFRSF17 & TRAF1 \\
\hline MAP3K5 & TRAF6 \\
\hline IFNG & IFNGR1 \\
\hline ANXA5 & COL10A1 \\
\hline$A R$ & CAV1 \\
\hline PMAIP1 & PPBP \\
\hline GFER & GFER \\
\hline APBA1 & PSEN1 \\
\hline CALB1 & IMPA1 \\
\hline CCNB1 & RALBP1 \\
\hline HIST2H2BE & HSPD1 \\
\hline CRMP1 & MRPS12 \\
\hline $\mathrm{CDH} 5$ & KDR \\
\hline RALGDS & RRAS \\
\hline DUSP1 & SKP2 \\
\hline MAPK3 & PTPN5 \\
\hline PECAM1 & PTPN11 \\
\hline DHX38 & PPP4C \\
\hline PRKAR2A & RAB32 \\
\hline FKBP1A & RYR1 \\
\hline EGFR & PRKCA \\
\hline NR3C1 & ONECUT1 \\
\hline FBLN2 & FBN1 \\
\hline CCL3 & CCL3 \\
\hline PPP2R1A & PPP2R5C \\
\hline BYSL & KRT8 \\
\hline $\mathrm{BCL} 2$ & MYC \\
\hline SNW1 & VDR \\
\hline SYK & TRAF6 \\
\hline IL6ST & STAT3 \\
\hline GNA13 & PTK2B \\
\hline EFNB1 & SDCBP \\
\hline $\mathrm{NCL}$ & SSB \\
\hline MYC & RBL1 \\
\hline F13A1 & F13A1 \\
\hline PLAU & SERPINA5 \\
\hline COPS6 & SLC2A1 \\
\hline E2F3 & TFE3 \\
\hline KPNB1 & PTHLH \\
\hline CCDC85B & KRT17 \\
\hline STK4 & STK4 \\
\hline EED & ITGAE \\
\hline CREBBP & SREBF2 \\
\hline NCOA1 & STAT3 \\
\hline IQGAP1 & RAC1 \\
\hline GBE1 & PGD \\
\hline HLA & LILRB1 \\
\hline
\end{tabular}




\begin{tabular}{|c|c|}
\hline SMN1 & TIAL1 \\
\hline CCND1 & NCOA1 \\
\hline NMI & STAT5B \\
\hline ABLIM1 & GOLGA2 \\
\hline IL2RB & STAT1 \\
\hline CD80 & CTLA4 \\
\hline FADD & NACA \\
\hline CDK4 & RFC1 \\
\hline FYN & SYK \\
\hline APOB & PPIB \\
\hline IGF1R & YWHAE \\
\hline DVL3 & PITX1 \\
\hline PI4KB & YWHAB \\
\hline KLKB1 & SERPINA5 \\
\hline PGAM1 & XRCC6 \\
\hline DAXX & HDAC2 \\
\hline ARHGAP5 & RHOA \\
\hline MYC & RAF1 \\
\hline CSF1R & YES1 \\
\hline EGFR & STAT1 \\
\hline COPS6 & RAB27A \\
\hline RAG1 & RAG2 \\
\hline PRTN3 & SERPINA1 \\
\hline MDFI & MDFI \\
\hline TAF1B & TP53 \\
\hline NFYB & TP53 \\
\hline EP300 & STAT1 \\
\hline GLI2 & SKI \\
\hline FMR1 & FXR2 \\
\hline PTPN11 & STAT5A \\
\hline NFKB2 & NFKB2 \\
\hline PML & RELA \\
\hline FHL2 & FHL2 \\
\hline HRAS & RASA1 \\
\hline BCL3 & KAT5 \\
\hline ITK & PPIA \\
\hline SETMAR & SETMAR \\
\hline RPS14 & TAF9 \\
\hline ЕНMT2 & LIMS1 \\
\hline TGFBR2 & TGFBR3 \\
\hline $\mathrm{A} 2 \mathrm{M}$ & LRP1 \\
\hline HSP90AA1 & STIP1 \\
\hline MAD2L1 & MAD2L1BP \\
\hline GHR & GRB10 \\
\hline PEX13 & PEX5 \\
\hline CALR & TF \\
\hline CNTFR & PLSCR1 \\
\hline
\end{tabular}




\begin{tabular}{|c|c|}
\hline CCDC85B & PSMF1 \\
\hline ESR1 & STAT5A \\
\hline SULT1E1 & UNC119 \\
\hline ERBB3 & MUC1 \\
\hline DLG1 & KCNJ6 \\
\hline NCK1 & PXN \\
\hline NCF2 & RAC2 \\
\hline HTT & TP53 \\
\hline FYN & VAV1 \\
\hline BAG1 & SIAH1 \\
\hline ARSA & ARSA \\
\hline EWSR1 & KEL \\
\hline HBEGF & MMP7 \\
\hline CCL7 & CCR3 \\
\hline CCND3 & PCNA \\
\hline GRB2 & KRT17 \\
\hline $\mathrm{F} 2 \mathrm{R}$ & GNA13 \\
\hline CCNE1 & SMARCA4 \\
\hline FGB & NID1 \\
\hline FYN & MAPT \\
\hline CASP8 & RALBP1 \\
\hline JUP & PKP2 \\
\hline HNRNPU & PRMT1 \\
\hline ATF2 & JUN \\
\hline ARL1 & GOLGA4 \\
\hline PAK2 & RAC1 \\
\hline DAP3 & DAP3 \\
\hline GAD1 & HAAO \\
\hline GABPB1 & LMO3 \\
\hline MDK & RPL18A \\
\hline RING1 & RING1 \\
\hline ELAVL3 & EWSR1 \\
\hline HDAC1 & SMAD2 \\
\hline INSR & KHDRBS1 \\
\hline ATXN1 & $\mathrm{GCA}$ \\
\hline CD81 & KIT \\
\hline NAP1L1 & TP53 \\
\hline F10 & F5 \\
\hline CTNNB1 & SMAD7 \\
\hline GNAQ & RGS7 \\
\hline DDR1 & SHC1 \\
\hline GCA & LCP1 \\
\hline PXN & TUBG1 \\
\hline GOLGA2 & GPS2 \\
\hline RAB5B & RIN1 \\
\hline ERCC3 & GTF2E1 \\
\hline CUL1 & GPS1 \\
\hline
\end{tabular}




\begin{tabular}{|c|c|}
\hline PRKCD & YWHAZ \\
\hline COPS6 & TK1 \\
\hline CDC37 & МАРЗКЗ \\
\hline HSPE1 & TLE1 \\
\hline MYB & PML \\
\hline MARK2 & SFN \\
\hline CYFIP2 & FMR1 \\
\hline DAZAP2 & PLSCR1 \\
\hline TSN & TSNAX \\
\hline FUS & SPI1 \\
\hline GPSM2 & HRAS \\
\hline GTF3C1 & MAPK10 \\
\hline S100A2 & S100A2 \\
\hline TBP & UBTF \\
\hline GMFB & MAPK3 \\
\hline BCL2A1 & HRK \\
\hline JAK3 & SHB \\
\hline PLK1 & PSMA1 \\
\hline TAF1 & TBP \\
\hline FHL2 & ITGB1 \\
\hline GNAO1 & RGS16 \\
\hline EEF1G & PTPN4 \\
\hline ERBB3 & PIK3R1 \\
\hline PPP2CA & PTN \\
\hline PPP4C & RELA \\
\hline CD19 & CD79A \\
\hline BRD8 & THRB \\
\hline DPM1 & ZBTB16 \\
\hline PLK1 & TP53 \\
\hline GRB2 & OCRL \\
\hline ATN1 & RUNX1T1 \\
\hline SH3GL1 & SH3GL3 \\
\hline DAB2 & SRC \\
\hline MNAT1 & POU2F2 \\
\hline ORC2L & RPA2 \\
\hline CREBBP & STAT3 \\
\hline KRT18 & PKP2 \\
\hline UROD & UROD \\
\hline RELA & STAT3 \\
\hline AP1B1 & CLTC \\
\hline CSF2RB & YWHAZ \\
\hline SKIL & XRCC6 \\
\hline EPS8 & ITGB3 \\
\hline ENO2 & UBE2C \\
\hline COL9A2 & FEZ1 \\
\hline SLC4A1 & SLC4A1 \\
\hline ATXN1 & TBC1D5 \\
\hline
\end{tabular}




\begin{tabular}{|c|c|}
\hline GTF2E2 & GTF2F2 \\
\hline PCNA & POLD1 \\
\hline GRB10 & KDR \\
\hline FYN & IL7R \\
\hline FYN & PAK2 \\
\hline CBL & $\mathrm{CBL}$ \\
\hline MATK & SRC \\
\hline CREB1 & SGK1 \\
\hline CYR61 & ITGB5 \\
\hline ELN & PRTN3 \\
\hline SMAD2 & ZMYND11 \\
\hline ENO2 & HSF1 \\
\hline ERBB3 & PIK3R2 \\
\hline S100A4 & TP53 \\
\hline GNB2 & GNG4 \\
\hline ССТ7 & CRMP1 \\
\hline ATR & CHD4 \\
\hline MARK2 & YWHAZ \\
\hline CTBP1 & HDAC2 \\
\hline MCM5 & STAT1 \\
\hline EMG1 & HIVEP1 \\
\hline NPPB & NPR3 \\
\hline IRS1 & UBTF \\
\hline MAP1B & SNCA \\
\hline RELA & SRF \\
\hline $\mathrm{BCR}$ & FES \\
\hline DOCK2 & RAC2 \\
\hline HMGB1 & RAG1 \\
\hline ESR1 & PRDM2 \\
\hline CCDC85B & MOBKL1B \\
\hline DLG4 & KCNA4 \\
\hline BARD1 & CSTF1 \\
\hline BCL6 & JUN \\
\hline$A R$ & FOXO1 \\
\hline ITGAD & ITGB2 \\
\hline F8 & PROS1 \\
\hline TK1 & UNC119 \\
\hline EEF1A1 & FAS \\
\hline POLR2E & TAF15 \\
\hline HMGB1 & NFKB1 \\
\hline CALR & CD1D \\
\hline CRK & NEDD9 \\
\hline NROB2 & RXRA \\
\hline TRIM9 & TRIM9 \\
\hline PDGFRA & PLCG1 \\
\hline DLG1 & GRIA1 \\
\hline NEDD4 & TP73 \\
\hline
\end{tabular}




\begin{tabular}{|c|c|}
\hline CDK2 & CHAF1A \\
\hline GLB1 & NEU1 \\
\hline ESR1 & PIK3R1 \\
\hline RUNX2 & XRCC6 \\
\hline PHB & RAF1 \\
\hline KLF10 & SP1 \\
\hline AKT1 & SMAD3 \\
\hline APLP1 & EIF2S2 \\
\hline CBX5 & XRCC6 \\
\hline MYH9 & MYL9 \\
\hline PDLIM7 & UBE2I \\
\hline COL4A5 & RNF10 \\
\hline ATF2 & MAPK14 \\
\hline CCNA1 & CDC25A \\
\hline GNB2L1 & STAT1 \\
\hline PTPN14 & TJP1 \\
\hline CCNA2 & SKP2 \\
\hline CASP3 & SREBF2 \\
\hline CNTF & CNTF \\
\hline CD19 & SYK \\
\hline PRKAR2A & RUNX1T1 \\
\hline ABL1 & MAP4K1 \\
\hline ABCA4 & CNGB1 \\
\hline CAV1 & TNFRSF1B \\
\hline PLAGL1 & SMAD3 \\
\hline SMARCA2 & SS18 \\
\hline$A R$ & $\mathrm{CCNH}$ \\
\hline CYBA & NCF2 \\
\hline CD247 & STAT5A \\
\hline PSAP & SMAD2 \\
\hline MAPK12 & SNTA1 \\
\hline CKS2 & EEF1A1 \\
\hline NME1 & TERF1 \\
\hline HDAC1 & SATB1 \\
\hline DHX9 & GTF2B \\
\hline MAGEA11 & TCEA2 \\
\hline ACVR1B & INHBA \\
\hline $\mathrm{HCK}$ & UNC119 \\
\hline PRKAR2A & RYR2 \\
\hline LCK & PXN \\
\hline EZR & FASLG \\
\hline COPS6 & COX5A \\
\hline BRCA1 & USF2 \\
\hline TRADD & TRAF3 \\
\hline MGMT & PRKACA \\
\hline NFKB2 & NFKBIE \\
\hline ESR1 & TBP \\
\hline
\end{tabular}




\begin{tabular}{|c|c|}
\hline SLC25A11 & SLC25A11 \\
\hline SCRIB & TJP2 \\
\hline HDAC1 & TP53 \\
\hline ACP5 & RPA2 \\
\hline HIP1 & RPS10 \\
\hline SMAD2 & UBA52 \\
\hline L1CAM & NUMB \\
\hline DSC3 & PKP4 \\
\hline PRKCZ & TRAF6 \\
\hline MCM2 & RPA2 \\
\hline SMAD7 & YAP1 \\
\hline GRB7 & INSR \\
\hline ATN1 & FBLN2 \\
\hline FGFR1 & PLCG1 \\
\hline EDNRA & KAT5 \\
\hline TGFBR1 & TGFBR2 \\
\hline SHC1 & ZAP70 \\
\hline EGR1 & EP300 \\
\hline AGTR1 & RAB5A \\
\hline CDX2 & PAX6 \\
\hline TAF9 & UBA1 \\
\hline APEX1 & HIF1A \\
\hline NCOR2 & RELA \\
\hline COL1A1 & TXN \\
\hline KPNA2 & KPNB1 \\
\hline ALOX12 & PLA2G2A \\
\hline JUNB & SMAD4 \\
\hline TNF & TNFRSF1A \\
\hline DES & SPTAN1 \\
\hline BMPR1A & BMPR1B \\
\hline PECAM1 & SRC \\
\hline CRYBB2 & CRYGC \\
\hline ELAVL4 & EWSR1 \\
\hline BIK & MCL1 \\
\hline BCL6 & NCOR2 \\
\hline CASP3 & DCC \\
\hline CREBBP & FOXO1 \\
\hline PECAM1 & PECAM1 \\
\hline WT1 & WTAP \\
\hline BRCA1 & RB1 \\
\hline ACTA2 & СCT4 \\
\hline CBX3 & LBR \\
\hline EWSR1 & MATK \\
\hline MAPT & YWHAB \\
\hline MAPK7 & SGK1 \\
\hline KAT2B & NPAS2 \\
\hline IL6ST & PTPN11 \\
\hline
\end{tabular}




\begin{tabular}{|c|c|}
\hline FTH1 & FTL \\
\hline CALR & FGB \\
\hline NR1H3 & PPARA \\
\hline SFN & ZNF638 \\
\hline AES & HMGB1 \\
\hline CREBBP & POLR2A \\
\hline PHB & SMARCA4 \\
\hline DLG2 & NOS1 \\
\hline DSC2 & DSG2 \\
\hline APC & СТВР1 \\
\hline ITGB3BP & ITGB5 \\
\hline FLNA & ITGB7 \\
\hline IFNAR1 & PIK3R1 \\
\hline BAT3 & TDGF1 \\
\hline CCDC6 & PPP4C \\
\hline CSTF2 & EEF1G \\
\hline DNMT1 & RB1 \\
\hline DHX9 & RELA \\
\hline NCOA1 & THRB \\
\hline CCR3 & HCK \\
\hline DCTN1 & RPGR \\
\hline ITGB1BP1 & KRIT1 \\
\hline NTRK3 & SHC1 \\
\hline AHR & NRIP1 \\
\hline EGR2 & HCFC1 \\
\hline UBE2D3 & UBE3A \\
\hline POLR2G & POLR2H \\
\hline PSME1 & PSME2 \\
\hline BRCA1 & BRCC3 \\
\hline $\mathrm{CDH} 8$ & CTNNB1 \\
\hline PTH & PTH1R \\
\hline JUND & MAPK1 \\
\hline FBN1 & MYOC \\
\hline ANP32A & APEX1 \\
\hline HNRNPH1 & NCBP2 \\
\hline $\mathrm{HGF}$ & HGF \\
\hline LYL1 & NFKB1 \\
\hline SMAD3 & SRY \\
\hline GZMA & HMGB2 \\
\hline TP73 & YAP1 \\
\hline GAST & MEP1B \\
\hline CTCF & POLR2A \\
\hline MMP2 & THBS1 \\
\hline NFYA & SRF \\
\hline CRKL & NEDD9 \\
\hline BCL2 & PPP3CA \\
\hline $\mathrm{KCNH} 2$ & YWHAE \\
\hline
\end{tabular}




\begin{tabular}{|c|c|}
\hline JUN & SPI1 \\
\hline CREBBP & NCOA2 \\
\hline HNRNPU & SMN1 \\
\hline DPEP1 & DPEP1 \\
\hline EXOSC7 & IP6K1 \\
\hline BCL2 & BCL2L1 \\
\hline HSP90AA1 & LSM1 \\
\hline ORC2L & XRCC5 \\
\hline SH3BP2 & SYK \\
\hline PPP2R3A & RBL1 \\
\hline MAP2K4 & MAPK8 \\
\hline NCOA6 & THRB \\
\hline RAB3A & RABGGTB \\
\hline MAPK1 & PRKCE \\
\hline SFN & SRRM2 \\
\hline POU2F2 & TBP \\
\hline CBL & YWHAB \\
\hline FGF7 & HSPG2 \\
\hline FYN & ITK \\
\hline CUL2 & TCEB1 \\
\hline GRIN2A & SRC \\
\hline CDK9 & GRN \\
\hline CFTR & PRKAA1 \\
\hline NCOR2 & PML \\
\hline HSPA5 & YWHAB \\
\hline CSNK1A1 & TNFRSF1B \\
\hline C1R & C1R \\
\hline EED & EZH1 \\
\hline MYC & MYCBP \\
\hline POLR2C & POLR2J \\
\hline CNTN2 & NFYB \\
\hline $\mathrm{FTL}$ & GADD45A \\
\hline FN1 & TSHR \\
\hline FMR1 & UBE2I \\
\hline GNAI1 & GPSM3 \\
\hline PRPSAP1 & PRPSAP1 \\
\hline DAPK1 & TNFRSF1A \\
\hline RAD9A & RPA1 \\
\hline EP300 & SMAD2 \\
\hline PSMC3 & PSMC5 \\
\hline ADRM1 & PSMD1 \\
\hline MAPK14 & MKNK1 \\
\hline LYN & PPP1R8 \\
\hline ATF6B & NFYC \\
\hline CDC6 & PCNA \\
\hline MDM2 & TBP \\
\hline BMPR1A & ZMYND11 \\
\hline
\end{tabular}




\begin{tabular}{|c|c|}
\hline CHGB & MARK3 \\
\hline CUL4B & NEDD8 \\
\hline DHFR & TP53 \\
\hline STX1A & STXBP1 \\
\hline CSNK2A1 & HDAC2 \\
\hline PRSS1 & SERPINB8 \\
\hline JUN & SPIB \\
\hline F13A1 & FN1 \\
\hline EIF2AK2 & PDGFRB \\
\hline CNTN2 & TUBG1 \\
\hline S100A8 & UNC119 \\
\hline ENG & TGFBR1 \\
\hline EPS8 & PCM1 \\
\hline RAD9A & RPA2 \\
\hline RAB5B & RIN2 \\
\hline ALB & LRP2 \\
\hline PECAM1 & PTPN6 \\
\hline HNRNPA2B1 & VHL \\
\hline KIF20B & PIN1 \\
\hline CCDC85B & NRIP1 \\
\hline PROC & PROCR \\
\hline SNRPG & TLE1 \\
\hline E2F1 & TP53BP1 \\
\hline RPGR & YWHAE \\
\hline KPNA2 & RAG1 \\
\hline HDAC2 & IKZF1 \\
\hline GP1BA & VWF \\
\hline EP300 & ETS1 \\
\hline BRCA1 & ESR1 \\
\hline FGF2 & RPL6 \\
\hline PNN & RNPS1 \\
\hline AHSG & INSR \\
\hline FXR2 & FXR2 \\
\hline PFN1 & VASP \\
\hline OCLN & TJP1 \\
\hline NCK1 & PAK2 \\
\hline RAB4A & TP53 \\
\hline KIF5B & YWHAZ \\
\hline IFNAR1 & PRMT1 \\
\hline CREBBP & HNF4A \\
\hline CALCOCO2 & FXR2 \\
\hline EEF1G & ZNF24 \\
\hline CAV2 & SRC \\
\hline CD28 & GRB2 \\
\hline PPP2R1B & PPP2R5E \\
\hline HTT & PDK2 \\
\hline ARL6IP1 & INPP5K \\
\hline
\end{tabular}




\begin{tabular}{|c|c|}
\hline JUN & MAPK3 \\
\hline NCOA1 & RXRA \\
\hline EGFR & GRB10 \\
\hline CD22 & LYN \\
\hline ITSN1 & SMARCC2 \\
\hline PLK1 & PSMA3 \\
\hline EWSR1 & MAGEA11 \\
\hline GTF2H1 & TP53 \\
\hline TRIM29 & UBE2I \\
\hline DMC1 & DMC1 \\
\hline $\mathrm{CDH} 11$ & $\mathrm{CDH} 2$ \\
\hline SLC25A6 & VIM \\
\hline CCDC85B & VPS72 \\
\hline AR & SART3 \\
\hline ITGA3 & ITGB1 \\
\hline EFNA1 & KAT5 \\
\hline EP300 & SMAD1 \\
\hline MCM4 & ORC2L \\
\hline BLM & CHAF1A \\
\hline CTNNB1 & PTPN14 \\
\hline CCDC85B & EPS8 \\
\hline CGA & CGB \\
\hline GOLGA2 & ZNF250 \\
\hline GRB14 & PDGFRB \\
\hline PCNA & RFC2 \\
\hline PUF60 & PUF60 \\
\hline ARHGAP19 & ATXN3 \\
\hline ELF3 & NFKB1 \\
\hline LDLR & LRPAP1 \\
\hline CREBBP & EWSR1 \\
\hline EP300 & NAP1L4 \\
\hline PSMD7 & TRAF6 \\
\hline CDK9 & SKP2 \\
\hline SNRPN & TP53 \\
\hline ABL1 & PRKDC \\
\hline DNM1 & GRB2 \\
\hline EPOR & PIK3R1 \\
\hline MAPRE1 & TERF1 \\
\hline IRAK1 & PRKCZ \\
\hline HSPA1A & MAP3K5 \\
\hline AHR & ESR1 \\
\hline CDK4 & CDKN2A \\
\hline CRADD & RIPK1 \\
\hline BIN1 & SYN1 \\
\hline ATR & $\mathrm{MSH} 2$ \\
\hline ATXN2 & BAT3 \\
\hline RIPK1 & TRAF1 \\
\hline
\end{tabular}




\begin{tabular}{|c|c|}
\hline LILRB4 & PTPN11 \\
\hline IGBP1 & PPP6C \\
\hline MDFI & TAP1 \\
\hline BAT3 & S100A4 \\
\hline PLD1 & SNCA \\
\hline KIT & YES1 \\
\hline$A R$ & TMF1 \\
\hline OCRL & RAB5A \\
\hline AKR1B1 & SMAD1 \\
\hline CCND1 & STAT3 \\
\hline $\mathrm{EZH} 2$ & VAV1 \\
\hline RPL7 & ZNF7 \\
\hline FN1 & GSN \\
\hline AFP & CCR5 \\
\hline PSMD2 & TNFRSF1A \\
\hline PLCG1 & PTPRJ \\
\hline FXR1 & FXR2 \\
\hline DGKD & HAX1 \\
\hline HRAS & PIK3R1 \\
\hline TAF10 & TAF10 \\
\hline CYFIP1 & FMR1 \\
\hline FLT3LG & PFN2 \\
\hline CRMP1 & SNRPG \\
\hline NKX2 & TBX5 \\
\hline GHR & PTPN11 \\
\hline ICAM3 & MSN \\
\hline CFTR & SLC9A3R2 \\
\hline CASP10 & CASP2 \\
\hline CDK2 & CEBPA \\
\hline EIF2AK2 & RAC1 \\
\hline TRIM23 & ZNF250 \\
\hline TAF11 & TAF5 \\
\hline CTTN & SYK \\
\hline CTBP1 & $\mathrm{HTT}$ \\
\hline TOMM20 & VDAC1 \\
\hline $\mathrm{C} 3$ & CFI \\
\hline SMAD3 & TGM2 \\
\hline HNF4A & NROB2 \\
\hline DNAJA1 & TM4SF1 \\
\hline HIST1H4A & PRMT5 \\
\hline EEF1A1 & SMAD2 \\
\hline MDC1 & TP53 \\
\hline GRIN2A & PTPN4 \\
\hline $\mathrm{CFH}$ & SELL \\
\hline NR3C1 & RAF1 \\
\hline SEC13 & SEC31A \\
\hline EGFR & PTK6 \\
\hline
\end{tabular}




\begin{tabular}{|c|c|}
\hline FLT3 & GRB2 \\
\hline SMAD1 & SMAD6 \\
\hline PTPN1 & STAT5B \\
\hline EPS8 & ITGB1 \\
\hline DAXX & MDM2 \\
\hline KDR & SHB \\
\hline PDGFB & PDGFB \\
\hline CREBBP & NEUROG1 \\
\hline FYN & GRIN2A \\
\hline NCOR2 & NR3C1 \\
\hline ANP32A & KPNA1 \\
\hline HSP90AA1 & NR3C1 \\
\hline EWSR1 & RAD23A \\
\hline KCNJ11 & LDHA \\
\hline BAD & $\mathrm{BCL} 2 \mathrm{~L} 2$ \\
\hline CDK6 & RUNX1 \\
\hline ALOX12 & ITGB4 \\
\hline$A X L$ & GAS6 \\
\hline HSP90AA1 & LCK \\
\hline FYB & FYN \\
\hline GOLGB1 & SKIL \\
\hline EEF1A1 & ITSN1 \\
\hline $\mathrm{CDH} 1$ & CTNNA1 \\
\hline KRT2 & SMAD3 \\
\hline ITPKB & PSME3 \\
\hline DHX9 & TBP \\
\hline FKBP4 & GLMN \\
\hline DHFR & THBS1 \\
\hline JAK1 & PLAUR \\
\hline HNF1A & STAT3 \\
\hline GRB10 & RET \\
\hline ITGA6 & ITGB1 \\
\hline HUWE1 & MCL1 \\
\hline CHAF1A & PCNA \\
\hline GNAS & VIPR1 \\
\hline HIF1A & SP1 \\
\hline HMGN2 & HMGN2 \\
\hline CBL & ITK \\
\hline DAP3 & PPARA \\
\hline SMAD3 & ST6GALNAC2 \\
\hline MAPK3 & SREBF2 \\
\hline ERCC2 & ERCC3 \\
\hline CCNG1 & CDKN2A \\
\hline GNAI2 & RGS19 \\
\hline LCP2 & NCK1 \\
\hline RAC1 & TRIO \\
\hline FANCA & FANCA \\
\hline
\end{tabular}




\begin{tabular}{|c|c|}
\hline SCAMP1 & SNAP23 \\
\hline CSF2RB & PRKCB \\
\hline KAT2B & MDM2 \\
\hline MAD2L1BP & PTN \\
\hline KRIT1 & RAP1A \\
\hline CLEC3B & HGF \\
\hline ESR1 & HSP90AA1 \\
\hline FOXO4 & XPO1 \\
\hline RELA & RPS3 \\
\hline HLA & SH3GL2 \\
\hline MDM4 & YWHAB \\
\hline$A R$ & $\mathrm{NR} 2 \mathrm{C} 2$ \\
\hline FBN1 & VCAN \\
\hline MMP2 & TIMP2 \\
\hline BLMH & RPL11 \\
\hline KIR2DL3 & PTPN6 \\
\hline DMWD & PPBP \\
\hline CREB1 & GLI2 \\
\hline PRKCD & PTPN6 \\
\hline $\mathrm{BCL} 2$ & PARP1 \\
\hline EPOR & GNB2L1 \\
\hline PPP2CA & PXN \\
\hline KLF6 & POLA2 \\
\hline KCNB1 & SNAP25 \\
\hline MITF & TFE3 \\
\hline CCDC85B & PSMC6 \\
\hline IGFBP3 & RXRA \\
\hline JUN & PRKD1 \\
\hline CHAF1A & CHAF1A \\
\hline GRK5 & RCVRN \\
\hline KAT5 & PTPN4 \\
\hline NFKB1 & REL \\
\hline POLR2J & SATB1 \\
\hline EZR & S100P \\
\hline PTPRC & SKAP1 \\
\hline FYN & PTPRA \\
\hline MAGEA1 & UQCRB \\
\hline BNIP3 & BNIP3 \\
\hline$A R$ & MAPK1 \\
\hline HSPB3 & PRPF4B \\
\hline RAP1A & RAPGEF5 \\
\hline IMPA1 & IMPA1 \\
\hline SMAD3 & TPM3 \\
\hline SMAD2 & TGFBR1 \\
\hline CREBBP & SRF \\
\hline PIK3R3 & RB1 \\
\hline GTF2E2 & TBP \\
\hline
\end{tabular}




\begin{tabular}{|c|c|}
\hline FHL2 & ITGA3 \\
\hline CFLAR & FADD \\
\hline PRTN3 & SERPINB1 \\
\hline PRDX1 & PRDX4 \\
\hline PSMD2 & UNC119 \\
\hline RB1 & SERPINB2 \\
\hline BAT3 & NBL1 \\
\hline ARHGEF12 & IGF1R \\
\hline PKN2 & RHOA \\
\hline TSC2 & YWHAZ \\
\hline$A R$ & XRCC6 \\
\hline KIT & PIK3R1 \\
\hline NOS2 & RAC2 \\
\hline CASP10 & CFLAR \\
\hline$A R$ & CREBBP \\
\hline CREBBP & SMAD2 \\
\hline GNB1 & GNG11 \\
\hline CTNNB1 & TFAP2A \\
\hline TPD52L1 & YWHAB \\
\hline CACNB4 & TBL3 \\
\hline KNG1 & KRT1 \\
\hline CHGB & YLPM1 \\
\hline CSRP3 & MYF6 \\
\hline CTNND1 & CTTN \\
\hline LMNB1 & PRKCA \\
\hline CRMP1 & FAS \\
\hline RB1 & RBBP5 \\
\hline ANK1 & SLC4A3 \\
\hline RAF1 & RBL2 \\
\hline SAFB & SRPK1 \\
\hline HMGB1 & TLE1 \\
\hline ARL4D & NDRG1 \\
\hline HLA & HLA \\
\hline FRK & RB1 \\
\hline CALM1 & GRK4 \\
\hline CDK2 & SKP2 \\
\hline NGFR & PRKACB \\
\hline RBBP8 & SIAH1 \\
\hline PRKCA & RALBP1 \\
\hline CIB1 & NBR1 \\
\hline NCOR2 & ZBTB16 \\
\hline TP53 & YBX1 \\
\hline ERBB4 & STAT5A \\
\hline GNAZ & RGS7 \\
\hline MCM2 & RPA1 \\
\hline NFIL3 & NFIL3 \\
\hline CDH5 & CTNND1 \\
\hline
\end{tabular}




\begin{tabular}{|c|c|}
\hline SMAD4 & TDG \\
\hline EP300 & MYOD1 \\
\hline PRKCB & RGS2 \\
\hline HDAC2 & PPP1R8 \\
\hline EIF2AK2 & STAT1 \\
\hline IL6ST & PTPN6 \\
\hline MAF & MAF \\
\hline МАР3К14 & TRAF1 \\
\hline APBB1 & APP \\
\hline ARF1 & KDELR1 \\
\hline KHDRBS1 & TUBB3 \\
\hline ARFRP1 & CYTH1 \\
\hline MYF5 & TCF3 \\
\hline ACTN1 & PKN1 \\
\hline HOXB6 & SAT1 \\
\hline ACADVL & ACADVL \\
\hline SP1 & YY1 \\
\hline CD46 & ITGB1 \\
\hline HDAC1 & RB1 \\
\hline CBL & EGFR \\
\hline PDLIM7 & PSMF1 \\
\hline LYL1 & TCF3 \\
\hline CDC20 & TCP1 \\
\hline RAD51 & SUMO1 \\
\hline ESR1 & MTA1 \\
\hline ATF3 & SMAD3 \\
\hline KPNA3 & $\mathrm{RCC1}$ \\
\hline MAPK1 & RPS6KA3 \\
\hline GLG1 & NFKB2 \\
\hline ICAM3 & ZBTB16 \\
\hline CTSK & SERPINB3 \\
\hline CREBBP & RPS6KA1 \\
\hline PPP2R1A & PPP4C \\
\hline BARD1 & BARD1 \\
\hline CTCF & RAD21 \\
\hline $\mathrm{A} 2 \mathrm{M}$ & NGF \\
\hline ARHGEF5 & SFN \\
\hline DOK1 & RET \\
\hline MBP & PLP1 \\
\hline FOXO4 & SMAD3 \\
\hline CLTC & KIT \\
\hline ERH & UNC119 \\
\hline IL2RB & SHC1 \\
\hline ELN & FBN1 \\
\hline AP3B1 & SLC30A3 \\
\hline ARF6 & ARFIP2 \\
\hline MAPK8 & WWOX \\
\hline
\end{tabular}




\begin{tabular}{|c|c|}
\hline PRKAA2 & PRKAB1 \\
\hline PXN & SRC \\
\hline EEF1A1 & TGIF1 \\
\hline FXR2 & NT5C2 \\
\hline SAT1 & SAT1 \\
\hline MYOG & SP1 \\
\hline AKAP1 & PRKAR2A \\
\hline GRIK1 & SDCBP \\
\hline NR2F6 & NR3C1 \\
\hline NR3C2 & PTGES3 \\
\hline PTN & PTPRS \\
\hline HIF1A & TP53 \\
\hline ACP1 & EPHB2 \\
\hline APOBEC1 & KPNA2 \\
\hline DAG1 & LAMA2 \\
\hline LRPPRC & NFKBIB \\
\hline DRP2 & DTNA \\
\hline GSK3B & YBX1 \\
\hline MDM4 & YWHAE \\
\hline ATXN1 & PIM2 \\
\hline MAP2K2 & MAPK3 \\
\hline COL1A1 & VWF \\
\hline RB1 & SKP2 \\
\hline EGR1 & SP1 \\
\hline HDAC1 & TOP2A \\
\hline DNAJC7 & SMAD2 \\
\hline DGKZ & SAT1 \\
\hline C1D & PRKDC \\
\hline CD58 & HERPUD1 \\
\hline CSF2RA & PIK3R1 \\
\hline MCL1 & PCNA \\
\hline ABLIM1 & KRT15 \\
\hline EZH2 & HDAC1 \\
\hline HSPE1 & SAT1 \\
\hline CD22 & SHC1 \\
\hline PSMD7 & RAB1A \\
\hline DSG3 & JUP \\
\hline MAGEA11 & PSMF1 \\
\hline ESR1 & SAFB2 \\
\hline SETDB1 & TTR \\
\hline RELB & RELB \\
\hline МАР3К14 & RPL4 \\
\hline HLA & LILRB1 \\
\hline TJP1 & TJP2 \\
\hline DUSP4 & MAPK3 \\
\hline IL4R & SHC1 \\
\hline SNAPC1 & SNAPC3 \\
\hline
\end{tabular}




\begin{tabular}{|c|c|}
\hline PCNA & YBX1 \\
\hline ADRBK1 & AGTR1 \\
\hline EWSR1 & HSPA2 \\
\hline DDX17 & DDX5 \\
\hline E4F1 & TP53 \\
\hline AKT1 & PPP2CA \\
\hline CDKN2A & PSMC3 \\
\hline NCK1 & WASL \\
\hline CDX2 & HNF1A \\
\hline CTNNB1 & PECAM1 \\
\hline $\mathrm{ABCD} 1$ & PEX19 \\
\hline ELF1 & SP1 \\
\hline KRT18 & RAF1 \\
\hline MED1 & TP53 \\
\hline PRKAB1 & PRKAG1 \\
\hline PTEN & PXN \\
\hline ASCL1 & TCF4 \\
\hline RAF1 & YWHAB \\
\hline E2F1 & PHB \\
\hline CTCF & SMC1A \\
\hline GNAI1 & RGS19 \\
\hline RAB5A & RABEP1 \\
\hline ATXN1 & SC4MOL \\
\hline TYK2 & XRCC5 \\
\hline MLLT10 & MLLT10 \\
\hline NFKBIA & REL \\
\hline$A X L$ & PIK3R2 \\
\hline CALM1 & MAPT \\
\hline ESR1 & NCOA1 \\
\hline GRB2 & KIT \\
\hline ARHGAP1 & RHOC \\
\hline PABPC1 & PCBP1 \\
\hline CDK9 & RELA \\
\hline ASMTL & ASMTL \\
\hline BAT2 & IFT88 \\
\hline PIK3R1 & SHB \\
\hline KAT2B & RELA \\
\hline ATP1A1 & MAPK3 \\
\hline RAB5A & TSC2 \\
\hline DAD1 & MCL1 \\
\hline GSK3B & MUC1 \\
\hline CD247 & ZAP70 \\
\hline KPNB1 & SMN1 \\
\hline FYN & GRB10 \\
\hline DNM1 & SRC \\
\hline AKAP9 & KCNQ1 \\
\hline SMAD4 & SP1 \\
\hline
\end{tabular}




\begin{tabular}{|c|c|}
\hline CDC37 & МАР3К14 \\
\hline PTEN & TP53 \\
\hline PROC & SERPINA5 \\
\hline EGFR & STAT3 \\
\hline$B M X$ & STAT3 \\
\hline COPS6 & EDN1 \\
\hline ATF2 & ATF2 \\
\hline CFTR & DNAJA1 \\
\hline NUP62 & TRAF3 \\
\hline ADH7 & $\mathrm{ADH7}$ \\
\hline FUS & THRA \\
\hline KIT & TEC \\
\hline ADRB2 & AKAP12 \\
\hline CNTN2 & NRCAM \\
\hline CREBBP & CTNNB1 \\
\hline PIN1 & RAB4A \\
\hline CBL & F2RL1 \\
\hline ABL1 & RFX1 \\
\hline GCG & GLP1R \\
\hline ID3 & PAX5 \\
\hline FCGR1A & $\mathrm{HCK}$ \\
\hline CRMP1 & PFN1 \\
\hline PXN & SELE \\
\hline SMAD1 & TOB1 \\
\hline MDM2 & PSME3 \\
\hline GRB2 & PTPN6 \\
\hline PIAS1 & SKIL \\
\hline NEDD9 & SMAD1 \\
\hline MEN1 & NFKB2 \\
\hline SETDB1 & SKIL \\
\hline MCM2 & MCM6 \\
\hline PIK3R1 & SYN1 \\
\hline AKR1C3 & MAGEA11 \\
\hline GDI1 & RAB9A \\
\hline BCL6 & ZBTB16 \\
\hline CLU & VLDLR \\
\hline GRK5 & GRK5 \\
\hline HSPG2 & TTR \\
\hline MAX & MYCL1 \\
\hline MDFI & ZNF136 \\
\hline CTSD & PSAP \\
\hline RAB5A & SDCBP \\
\hline PLG & S100A10 \\
\hline HERPUD1 & USP7 \\
\hline MCM7 & RPA1 \\
\hline ACVR1 & FNTA \\
\hline RB1 & TGM2 \\
\hline
\end{tabular}




\begin{tabular}{|c|c|}
\hline PRMT5 & SUPT5H \\
\hline RAD52 & RPA1 \\
\hline MARK2 & YWHAB \\
\hline IL6ST & PRKCD \\
\hline FCER1G & SYK \\
\hline SCNN1A & UBE2I \\
\hline PPFIA1 & PTPRF \\
\hline LMO2 & TAL1 \\
\hline PAX6 & SMARCA4 \\
\hline CTNND1 & PTPRM \\
\hline CREBBP & FGFR1 \\
\hline BRAF & HRAS \\
\hline ARF3 & ARFIP2 \\
\hline RNF4 & TCF20 \\
\hline KPNB1 & SREBF2 \\
\hline CBS & CBS \\
\hline ANXA5 & ITGB5 \\
\hline RPA1 & RPA3 \\
\hline GHR & PIK3R1 \\
\hline CLEC3B & PLAT \\
\hline AXIN1 & CSNK1E \\
\hline NCOA2 & NR3C1 \\
\hline EP300 & MYB \\
\hline CDH5 & PTPRB \\
\hline MCM2 & TBP \\
\hline PSMD11 & UNC119 \\
\hline BTF3 & CSNK2B \\
\hline GRIA2 & SDCBP \\
\hline CBX1 & DNMT1 \\
\hline LCK & LCP2 \\
\hline BRCA1 & MED21 \\
\hline HSPA4 & TRAF6 \\
\hline PCNA & PCNA \\
\hline POLR2B & WWOX \\
\hline NCOA1 & RARB \\
\hline GP1BA & SELP \\
\hline EGFR & GRB2 \\
\hline COL3A1 & THBS1 \\
\hline COL4A1 & COL4A1 \\
\hline GNAI3 & RGS19 \\
\hline EIF4G2 & SKIL \\
\hline$A R$ & TCF4 \\
\hline TP73 & WWOX \\
\hline HSF1 & XRCC5 \\
\hline GATA1 & MED1 \\
\hline ABL1 & $\mathrm{CBL}$ \\
\hline PFN1 & WASL \\
\hline
\end{tabular}




\begin{tabular}{|c|c|}
\hline CSTF2 & $\mathrm{ECH} 1$ \\
\hline$A X L$ & LCK \\
\hline ASAH1 & SETDB1 \\
\hline BAT1 & RPS15A \\
\hline BRCA1 & KPNA2 \\
\hline CLU & TGFBR2 \\
\hline IGF1R & IGFBP3 \\
\hline AR & NCOA4 \\
\hline FKBP1A & YY1 \\
\hline CD36 & MATK \\
\hline PTPN2 & SFN \\
\hline KDR & RASA1 \\
\hline BTG1 & PRMT1 \\
\hline SELP & VCAN \\
\hline C1QBP & YWHAB \\
\hline DRD3 & GRB2 \\
\hline GNB2L1 & SRC \\
\hline RPLP1 & XRCC6 \\
\hline STX3 & VAMP3 \\
\hline ARHGDIA & RAC1 \\
\hline ABCE1 & RNASEL \\
\hline HNRNPA1 & SAFB \\
\hline HRAS & RAF1 \\
\hline CREBBP & KLF5 \\
\hline EEF1G & HMOX2 \\
\hline CRKL & MAP4K1 \\
\hline PEX5 & PEX7 \\
\hline TRIM28 & ZNF197 \\
\hline $\mathrm{ECH} 1$ & PAFAH1B3 \\
\hline NFKB1 & RELB \\
\hline CALB1 & TRPV6 \\
\hline FYN & PIK3R1 \\
\hline GP1BA & YWHAZ \\
\hline PML & STAT3 \\
\hline AXIN1 & DVL1 \\
\hline ANG & RNH1 \\
\hline MAPK1 & MAPK14 \\
\hline CFHR4 & CFHR4 \\
\hline BLMH & BLMH \\
\hline APC & SIAH1 \\
\hline ARNTL & EPAS1 \\
\hline PSMB5 & TRAF6 \\
\hline AXL & $\mathrm{CBL}$ \\
\hline LYN & NMT1 \\
\hline ITGA9 & ITGB1 \\
\hline CD80 & CD80 \\
\hline PEX19 & SMAD2 \\
\hline
\end{tabular}




\begin{tabular}{|c|c|}
\hline CTNNB1 & MUC1 \\
\hline PLSCR1 & SPG7 \\
\hline ARL3 & TP53 \\
\hline PCNA & PRKDC \\
\hline GNB2L1 & ITGB1 \\
\hline MAPK7 & PTPRR \\
\hline PSEN1 & TCF7L2 \\
\hline HDAC1 & RBBP7 \\
\hline BRCA1 & SP1 \\
\hline KLK2 & SERPINF2 \\
\hline CASP3 & PARG \\
\hline ERCC2 & GTF2H2 \\
\hline E2F1 & TFDP1 \\
\hline POLR2A & POLR2H \\
\hline BTK & EWSR1 \\
\hline CYR61 & ITGAV \\
\hline AGA & $A G A$ \\
\hline $\mathrm{F} 2$ & THBD \\
\hline CDK2 & CDKN1A \\
\hline PDHX & PDK2 \\
\hline PIK3R1 & WAS \\
\hline MCM3 & MCM7 \\
\hline DYNLT3 & VDAC1 \\
\hline FYN & NMT1 \\
\hline MYOD1 & STAT3 \\
\hline SELP & SERPING1 \\
\hline FBN2 & MATN2 \\
\hline EIF2AK2 & PPP2R5A \\
\hline RAF1 & RB1 \\
\hline GRB7 & RET \\
\hline ADRBK1 & PDE6G \\
\hline AKT1 & CASP3 \\
\hline E2F1 & SP4 \\
\hline NFKBIB & POLR2L \\
\hline TGFB1 & TGFBR2 \\
\hline PLN & PPP1R3A \\
\hline EEF1D & EEF1G \\
\hline ССТ3 & RAF1 \\
\hline IMMT & KIF22 \\
\hline ATXN1 & BAT2 \\
\hline NME1 & RRAD \\
\hline CASP3 & TRAF3 \\
\hline CTNNB1 & DVL3 \\
\hline CCL8 & VCAN \\
\hline NR3C1 & STAT5B \\
\hline COPS2 & IRF8 \\
\hline GNAQ & RGS4 \\
\hline
\end{tabular}




\begin{tabular}{|c|c|}
\hline DLG4 & PTPRG \\
\hline PIN1 & TOP2A \\
\hline MAP3K5 & MAР3К5 \\
\hline CSNK2A1 & IRF2 \\
\hline ARHGDIB & RAC2 \\
\hline $\mathrm{APC}$ & MUC1 \\
\hline $\mathrm{ECH} 1$ & SERPINB9 \\
\hline APBB2 & APP \\
\hline POLR2C & POLR2H \\
\hline STX1A & SYT1 \\
\hline EPHA2 & PTPN11 \\
\hline HK3 & LEP \\
\hline ESR1 & NRIP1 \\
\hline BAG1 & HSPA1A \\
\hline HMGB2 & POU5F1 \\
\hline BRCA1 & JAK1 \\
\hline CBL & TYK2 \\
\hline ESR1 & JUN \\
\hline GNA13 & MCF2 \\
\hline PAFAH1B3 & ZBTB16 \\
\hline ADRB2 & GRK6 \\
\hline CCDC85B & POLR2L \\
\hline BCL2L1 & PSEN1 \\
\hline GNAI1 & RGS16 \\
\hline PML & ZBTB16 \\
\hline EEF1G & GSTO1 \\
\hline SNAP23 & VAMP3 \\
\hline GRB2 & PTPRA \\
\hline MAPT & PSEN1 \\
\hline TERF1 & TERF1 \\
\hline IKZF1 & TBP \\
\hline MCM7 & ORC3L \\
\hline MDM2 & NUMB \\
\hline RAB5A & RIN2 \\
\hline CYB5A & CYCS \\
\hline IRF2 & КАТ2B \\
\hline GDI2 & RAB2A \\
\hline S100A8 & S100A8 \\
\hline PLAUR & TYK2 \\
\hline TAL1 & TCF3 \\
\hline NID1 & SKIL \\
\hline $\mathrm{APC}$ & $\mathrm{APC}$ \\
\hline SERPINE1 & SERPINE1 \\
\hline MAN2A2 & VIM \\
\hline $\mathrm{HCK}$ & RAPGEF1 \\
\hline CACNA1C & SRI \\
\hline $\mathrm{MSH} 2$ & MSH3 \\
\hline
\end{tabular}




\begin{tabular}{|c|c|}
\hline PSMA3 & SNRPB \\
\hline CFTR & SNAP23 \\
\hline AP3B2 & CLTC \\
\hline RXRG & VDR \\
\hline LMNB1 & PRKCB \\
\hline LYST & YWHAB \\
\hline DDX3X & NFKB2 \\
\hline CDC7 & CDKN1A \\
\hline BRCA1 & FHL2 \\
\hline CASP3 & HCLS1 \\
\hline CEACAM5 & EWSR1 \\
\hline BRCA1 & TUBG1 \\
\hline ERBB2 & HSP90AA] \\
\hline CCDC85B & SMARCD1 \\
\hline NCOR2 & SP1 \\
\hline HSPD1 & HSPE1 \\
\hline GRIA4 & PRKACA \\
\hline CTNND1 & PTPRJ \\
\hline DVL1 & DVL3 \\
\hline IRS1 & PIK3R1 \\
\hline IFNAR1 & STAT3 \\
\hline EPS15 & NUMB \\
\hline HMGB1 & TP53 \\
\hline MYC & RELA \\
\hline CSK & ERBB3 \\
\hline MCM7 & MNAT1 \\
\hline NROB1 & RORA \\
\hline TAF6 & TAF7 \\
\hline F8 & VWF \\
\hline ACP1 & EPHB1 \\
\hline NME1 & TIAM1 \\
\hline DAG1 & RAPSN \\
\hline PECAM1 & PLCG1 \\
\hline $\mathrm{BIRC2}$ & JUP \\
\hline BIRC2 & CASP3 \\
\hline BMX & PAK1 \\
\hline LGALS1 & LGALS3BP \\
\hline HNF4A & ZNHIT3 \\
\hline GRIA1 & SDCBP \\
\hline F5 & PROS1 \\
\hline EEF1A1 & POLR2C \\
\hline CRYAA & CRYGC \\
\hline GAPDH & RPA2 \\
\hline PUM1 & SMAD1 \\
\hline DCC & MAPK3 \\
\hline DAP3 & FADD \\
\hline HSP90AA1 & MAP3К14 \\
\hline
\end{tabular}




\begin{tabular}{|c|c|}
\hline NFKB1 & NFKBIE \\
\hline AKAP13 & YWHAB \\
\hline AKAP13 & RHOA \\
\hline FADD & RIPK1 \\
\hline CCNB1 & $\mathrm{CDC} 25 \mathrm{~A}$ \\
\hline DOK1 & TEC \\
\hline JUN & NR5A1 \\
\hline ADAM15 & SRC \\
\hline PPBP & PPBP \\
\hline PRMT5 & TYK2 \\
\hline NR1H2 & RXRA \\
\hline ABL1 & VAV1 \\
\hline S100A11 & S100B \\
\hline MCM6 & PSMA1 \\
\hline ATM & TERF1 \\
\hline IGF1R & YWHAB \\
\hline CADPS & PIP5K1A \\
\hline CDC25C & YWHAH \\
\hline EEF1A1 & MNAT1 \\
\hline ABL1 & $\mathrm{BCR}$ \\
\hline AHR & NCOA2 \\
\hline BNIP1 & NCBP1 \\
\hline CCNB1 & CDKN1A \\
\hline ITGB3 & PTK2B \\
\hline CLTC & GGA3 \\
\hline HMGA2 & RELA \\
\hline SKAP1 & SRC \\
\hline PIK3R1 & SYK \\
\hline G3BP1 & USP10 \\
\hline MPRIP & YWHAQ \\
\hline COASY & RPS6KB1 \\
\hline PDK1 & RPS6KB1 \\
\hline ITSN1 & KIF5A \\
\hline MAD1L1 & MAD1L1 \\
\hline CCL5 & CCR4 \\
\hline CXCL12 & CXCL12 \\
\hline BARD1 & TP53 \\
\hline PIAS1 & SMAD1 \\
\hline CGA & FSHB \\
\hline DLG2 & GRIN2B \\
\hline ADD2 & FYN \\
\hline$A R$ & SMAD3 \\
\hline $\mathrm{CDH} 1$ & ITGAE \\
\hline LYST & YWHAQ \\
\hline ADAM9 & PRKCD \\
\hline MDFI & VPS72 \\
\hline EWSR1 & PRUNE2 \\
\hline
\end{tabular}




\begin{tabular}{|c|c|}
\hline PPP2R5D & PPP4C \\
\hline EPS8 & SRC \\
\hline ADRBK1 & SNCA \\
\hline TOP2A & TP53 \\
\hline ISG15 & UBA7 \\
\hline ESR1 & NROB2 \\
\hline ANXA2 & S100A10 \\
\hline IRF3 & IRF7 \\
\hline EED & $\mathrm{EZH} 2$ \\
\hline EP300 & EP300 \\
\hline AHDC1 & ATXN1 \\
\hline NCOA6 & THRA \\
\hline MDFI & MYF5 \\
\hline $\mathrm{BCL} 2$ & PSEN1 \\
\hline CDC42EP1 & RBPMS \\
\hline PRKCG & YWHAE \\
\hline DLG4 & HTR2C \\
\hline CRMP1 & HTT \\
\hline APCS & FCGR3B \\
\hline $\mathrm{F} 2 \mathrm{R}$ & SNX1 \\
\hline MCM7 & RBL1 \\
\hline ABL1 & ABL1 \\
\hline $\mathrm{BCL} 3$ & NFKB2 \\
\hline BAG1 & HBEGF \\
\hline KRT15 & PSMA1 \\
\hline MATN2 & MATN2 \\
\hline PLSCR1 & SLPI \\
\hline$A R$ & CDC37 \\
\hline ACTG1 & ACTG1 \\
\hline CRMP1 & FUBP1 \\
\hline NCK1 & RRAS \\
\hline HSP90AA1 & RPS3A \\
\hline CREB1 & NR3C1 \\
\hline GRB2 & PAK2 \\
\hline PARP1 & PARP1 \\
\hline CCT5 & TP53 \\
\hline APBA1 & APP \\
\hline CREBBP & EP300 \\
\hline PSMB6 & PSMB7 \\
\hline PRKD1 & SYK \\
\hline PTGS2 & TP53 \\
\hline EIF2AK2 & EIF2S1 \\
\hline PRKCG & RGS2 \\
\hline ILK & ITGB1 \\
\hline BCR & ERCC3 \\
\hline МАР3К4 & PTK2B \\
\hline CD22 & PTPN6 \\
\hline
\end{tabular}




\begin{tabular}{|c|c|}
\hline MED1 & PPARG \\
\hline BRCA1 & SMAD3 \\
\hline SYN1 & SYN1 \\
\hline HNRNPU & SFN \\
\hline KRT15 & ZNF638 \\
\hline CRYAA & CRYAB \\
\hline POU2F2 & POU2F2 \\
\hline CD81 & CD81 \\
\hline OGT & OGT \\
\hline COL1A1 & MATN2 \\
\hline ATXN1 & UBAP2L \\
\hline CUL3 & NEDD8 \\
\hline ANXA2 & CTSB \\
\hline GRB2 & TNK2 \\
\hline SMAD3 & SNW1 \\
\hline CRHR1 & UCN \\
\hline ATF4 & TTR \\
\hline TGFB1 & TGFB2 \\
\hline CLTA & HIP1 \\
\hline PPIA & S100A8 \\
\hline ACVR1B & SNX1 \\
\hline IL2RG & JAK3 \\
\hline PDGFB & PDGFRA \\
\hline C9 & CLU \\
\hline MNAT1 & MTA1 \\
\hline C1QBP & COIL \\
\hline G3BP1 & RASA1 \\
\hline CALCOCO2 & VPS72 \\
\hline CBL & LCK \\
\hline C3 & $\mathrm{CFH}$ \\
\hline HNRNPC & KPNA3 \\
\hline BCL2L1 & BIK \\
\hline PARP1 & RARA \\
\hline BARD1 & PTN \\
\hline FGF1 & S100A13 \\
\hline AHR & TBP \\
\hline KRT15 & PSMC5 \\
\hline CCDC130 & EEF1A1 \\
\hline ADAM15 & YES1 \\
\hline CTNNB1 & RXRA \\
\hline XRCC4 & XRCC4 \\
\hline CDK4 & CDKN1A \\
\hline SP3 & TP53 \\
\hline PML & SKI \\
\hline JUN & STAT3 \\
\hline ACTN1 & COIL \\
\hline ATP5C1 & СCT7 \\
\hline
\end{tabular}




\begin{tabular}{|c|c|}
\hline HMGB2 & PGR \\
\hline MAPK3 & PTPRR \\
\hline ARAF & RABGGTB \\
\hline GJA1 & PRKCA \\
\hline GTF3C1 & KLF6 \\
\hline SH3GL3 & VIM \\
\hline MNAT1 & RB1 \\
\hline PARK7 & PARK7 \\
\hline PRKACA & PRKAR1A \\
\hline CITED2 & TFAP2A \\
\hline AKAP13 & ESR1 \\
\hline FLNB & TSHR \\
\hline CD9 & SERPINH1 \\
\hline NEDD9 & SMAD2 \\
\hline EIF3A & EIF4G1 \\
\hline GRB2 & ZAP70 \\
\hline BAT2 & UBAP2L \\
\hline HNRNPA1 & PRMT1 \\
\hline IL12RB2 & STAT4 \\
\hline GOLGB1 & PFN2 \\
\hline FOSL1 & USF1 \\
\hline HDAC2 & SP1 \\
\hline APLP1 & HSPE1 \\
\hline CREBBP & RBBP4 \\
\hline ADAM15 & FYN \\
\hline MYC & SKP2 \\
\hline CSK & DAB2 \\
\hline RPA2 & STAT3 \\
\hline ARL6IP1 & FDFT1 \\
\hline DCTN1 & VIM \\
\hline SETDB1 & TRIM28 \\
\hline IGFBP5 & SPP1 \\
\hline IKBKE & TANK \\
\hline MLH1 & MLH3 \\
\hline TAF11 & TAF7 \\
\hline NOV & POLR2L \\
\hline LMNA & SREBF1 \\
\hline SMAD5 & SNRPA \\
\hline CASP3 & YWHAE \\
\hline ATM & RBBP8 \\
\hline GEM & TRIM23 \\
\hline NKRF & RELA \\
\hline NR4A1 & RXRG \\
\hline LRPAP1 & POLA2 \\
\hline PLCG1 & SHB \\
\hline TAF12 & TAF4 \\
\hline INSM1 & TUBB3 \\
\hline
\end{tabular}




\begin{tabular}{|c|c|}
\hline FLNA & TTN \\
\hline SH3GL1 & SH3GL1 \\
\hline BYSL & TROAP \\
\hline GRN & SLPI \\
\hline SCNN1B & WWP2 \\
\hline LIFR & PTPN6 \\
\hline MC1R & POMC \\
\hline CSH1 & SMAD4 \\
\hline FXR2 & TRIM29 \\
\hline PNN & PPIG \\
\hline BCR & GRB10 \\
\hline HIP1 & HTT \\
\hline PITX2 & PITX2 \\
\hline SSBP1 & SSBP1 \\
\hline CBL & LTK \\
\hline DRD2 & SLC6A3 \\
\hline PXN & VCL \\
\hline LCK & MAPK1 \\
\hline LYN & SKAP1 \\
\hline COL2A1 & TGFB1 \\
\hline IGFBP3 & TF \\
\hline SELP & SELPLG \\
\hline POLR2G & VHL \\
\hline RIPK1 & TNFAIP3 \\
\hline NEDD8 & PSMD4 \\
\hline ABL1 & TUB \\
\hline PSMC2 & SKIL \\
\hline TPD52 & TPD52L1 \\
\hline ATF1 & GABPA \\
\hline ACAN & LUM \\
\hline SF3A1 & SMAD3 \\
\hline STX1A & VAMP1 \\
\hline BCL2 & SMN1 \\
\hline EP300 & MYBL2 \\
\hline PIN1 & PTOV1 \\
\hline AES & RELA \\
\hline $\mathrm{HCK}$ & PAK2 \\
\hline APLP1 & ZNF24 \\
\hline MAP2K1 & MAPK1 \\
\hline $\mathrm{NCL}$ & TERT \\
\hline RB1 & TBP \\
\hline $\mathrm{CDH} 1$ & PSEN1 \\
\hline RARA & RXRB \\
\hline GRB7 & KIT \\
\hline PDGFRB & PIK3R1 \\
\hline CD247 & SLA \\
\hline MAD1L1 & MAD2L1 \\
\hline
\end{tabular}




\begin{tabular}{|c|c|}
\hline LCK & SYK \\
\hline OS9 & SMAD2 \\
\hline TPSAB1 & TPSAB1 \\
\hline PITX1 & POU1F1 \\
\hline BCL2L2 & HRK \\
\hline EBI3 & SMAD3 \\
\hline AKT1 & AKT1 \\
\hline COX17 & cox17 \\
\hline AR & GAPDH \\
\hline DLG1 & GRIN2A \\
\hline $\mathrm{P} 4 \mathrm{HB}$ & TG \\
\hline BLOC1S1 & PBX2 \\
\hline MLH1 & TRIM29 \\
\hline GAPDH & TK1 \\
\hline AKT1 & TCL1A \\
\hline RXRA & SP1 \\
\hline CSF2 & CSF2RA \\
\hline GNB2L1 & IL4R \\
\hline $\mathrm{BCL} 2$ & BECN1 \\
\hline BRAF & YWHAQ \\
\hline EEF1A1 & SMAD4 \\
\hline MDFI & PHLDA1 \\
\hline TFAP2C & TP53 \\
\hline FCN1 & TGFB1 \\
\hline DLG1 & KCNA5 \\
\hline C1S & SERPING1 \\
\hline COIL & MAD1L1 \\
\hline LCK & UNC119 \\
\hline DES & S100A1 \\
\hline EYA2 & GNAZ \\
\hline CALR & NKX2 \\
\hline AR & FHL2 \\
\hline CDC25A & MAP3K5 \\
\hline ARL4D & PGAM1 \\
\hline EFNA5 & ЕРНA3 \\
\hline CEACAM1 & CEACAM 8 \\
\hline CCDC106 & SETDB1 \\
\hline FGF2 & GPC3 \\
\hline AES & CCL7 \\
\hline SREBF2 & UBE2I \\
\hline CREBBP & ONECUT1 \\
\hline $\mathrm{FHL2}$ & PSEN2 \\
\hline CCL22 & VCAM1 \\
\hline CASP8 & RIPK1 \\
\hline FLNB & PSEN2 \\
\hline CUL1 & SKP2 \\
\hline LIG3 & XRCC1 \\
\hline
\end{tabular}




\begin{tabular}{|c|c|}
\hline RIN1 & YWHAE \\
\hline ELK1 & KLF4 \\
\hline CD163 & CSNK2B \\
\hline ESR1 & MED1 \\
\hline RFX1 & SMAD1 \\
\hline BAD & S100A10 \\
\hline PUF60 & VIM \\
\hline FOS & TSC22D3 \\
\hline EZR & ICAM2 \\
\hline SP100 & UBE2I \\
\hline CALM1 & PTH2R \\
\hline JUN & UBE2I \\
\hline CSTB & CTSH \\
\hline МАР3К8 & NFKB1 \\
\hline CALR & PDIA3 \\
\hline MYC & RB1 \\
\hline CUL4A & DDB2 \\
\hline DLAT & PDHX \\
\hline RPL35A & SH3GL3 \\
\hline EP300 & HIF1A \\
\hline MAPK3 & MKNK1 \\
\hline CRKL & MAP4K5 \\
\hline ADORA2B & SLC9A3R2 \\
\hline NKX2 & RELA \\
\hline CCNA1 & GPS2 \\
\hline CCNA1 & XRCC6 \\
\hline MDM4 & TP53 \\
\hline HTATSF1 & SUPT5H \\
\hline $\mathrm{ABI} 2$ & KRT20 \\
\hline ABL1 & PAK2 \\
\hline PLK1 & TSC1 \\
\hline DLG4 & KCNJ2 \\
\hline IL6ST & JAK1 \\
\hline LRP2 & LRPAP1 \\
\hline RARB & RXRA \\
\hline GATA4 & SRF \\
\hline CDC20 & HDAC1 \\
\hline ENO1 & PLG \\
\hline CSE1L & HNRNPL \\
\hline DLG4 & NOS1 \\
\hline NXF1 & SFRS3 \\
\hline FGA & FGA \\
\hline CDKN2A & MDM2 \\
\hline JUN & TGIF1 \\
\hline MEF2C & SMAD2 \\
\hline CITED2 & TFAP2C \\
\hline TP53 & ZNF148 \\
\hline
\end{tabular}




\begin{tabular}{|c|c|}
\hline ССТ3 & PAFAH1B2 \\
\hline STX3 & STXBP1 \\
\hline ANP32A & SET \\
\hline CSF2RB & CSF2RB \\
\hline STX1A & STX1A \\
\hline CBL & PIK3R1 \\
\hline RELA & RELA \\
\hline EP300 & USF2 \\
\hline CCL7 & FEZ1 \\
\hline FASLG & NCK1 \\
\hline PTK2B & PTPN12 \\
\hline RPS6KB1 & XPO1 \\
\hline CDC27 & SMAD3 \\
\hline DRD5 & GABRG2 \\
\hline JUN & SUMO1 \\
\hline CCL5 & SDC1 \\
\hline COL2A1 & PKD1 \\
\hline CBL & ZAP70 \\
\hline SEMA3F & UBA1 \\
\hline CCK & CCKAR \\
\hline CAV1 & ESR1 \\
\hline MDM4 & SFN \\
\hline DLG1 & LCK \\
\hline TAF1A & TBP \\
\hline CTTN & WIPF1 \\
\hline PABPC1 & wWOX \\
\hline CFLAR & TRAF1 \\
\hline PSMC2 & TRAF6 \\
\hline TNFAIP3 & TNFAIP3 \\
\hline KPNA1 & TAF9 \\
\hline DAG1 & GRB2 \\
\hline TAF1C & TBP \\
\hline DLG1 & EPB41 \\
\hline ERBB2 & GRB7 \\
\hline ANXA6 & S100A1 \\
\hline CCNA1 & GNB2L1 \\
\hline DGKZ & SNTB2 \\
\hline HNRNPF & HNRNPH1 \\
\hline EP300 & MDM2 \\
\hline TRIM9 & VASP \\
\hline CSRP1 & CSRP1 \\
\hline EWSR1 & PCBP1 \\
\hline$A R$ & DAXX \\
\hline POLR2C & POLR2C \\
\hline TERF1 & ZBTB16 \\
\hline LYN & PTPRC \\
\hline SMAD4 & SMAD5 \\
\hline
\end{tabular}




\begin{tabular}{|c|c|}
\hline PLSCR1 & ZBTB16 \\
\hline CGA & LHCGR \\
\hline$A R$ & PRMT1 \\
\hline GHR & STAT5B \\
\hline NME4 & TRIP13 \\
\hline GAP43 & RABEP1 \\
\hline DDX5 & ESR1 \\
\hline FKBP3 & HDAC2 \\
\hline BARD1 & EWSR1 \\
\hline EIF2AK2 & HSP90AA1 \\
\hline MTMR2 & MTMR2 \\
\hline RBBP4 & SP1 \\
\hline PIAS1 & UBE2I \\
\hline APEX1 & HNRNPL \\
\hline NEUROD2 & PKN1 \\
\hline ERBB2 & STAT3 \\
\hline FLNA & MAPK14 \\
\hline CCND1 & INSM1 \\
\hline MAML1 & NOTCH3 \\
\hline USP7 & USP7 \\
\hline FBP1 & FXR2 \\
\hline LIG4 & PRKDC \\
\hline GNB1 & GNGT1 \\
\hline EEF1A1 & TTR \\
\hline BCL6 & EP300 \\
\hline МАРЗКЗ & YWHAE \\
\hline CBX3 & TRIM28 \\
\hline MPP3 & SF3B3 \\
\hline ANXA2 & GRB2 \\
\hline CRMP1 & SEPHS1 \\
\hline SAFB & SAFB2 \\
\hline HSP90AA1 & TERT \\
\hline TAF5 & TAF9 \\
\hline KIF23 & SFN \\
\hline CEBPZ & TP53 \\
\hline CHN2 & RAC1 \\
\hline NCK1 & RASA1 \\
\hline AGFG1 & EPS15 \\
\hline ARHGDIA & RHOA \\
\hline ID1 & TCF4 \\
\hline MAST1 & SNTB2 \\
\hline ABL1 & BRCA1 \\
\hline EGFR & RIPK1 \\
\hline CHRNA1 & CHRND \\
\hline COPS6 & PAFAH1B3 \\
\hline SMAD2 & SP1 \\
\hline IRS1 & YWHAB \\
\hline
\end{tabular}




\begin{tabular}{|c|c|}
\hline HCFC1 & MLL \\
\hline TARBP2 & TARBP2 \\
\hline CSNK2A1 & YWHAB \\
\hline TAF1A & TAF1B \\
\hline APBB1 & TFCP2 \\
\hline ANXA3 & EMG1 \\
\hline CCL7 & CCR1 \\
\hline GNA11 & RGS3 \\
\hline PCBP2 & SFRS3 \\
\hline EIF4A2 & EIF4G1 \\
\hline MPHOSPH6 & TLE1 \\
\hline GAPDH & PDIA2 \\
\hline SFN & TRIM32 \\
\hline NCSTN & PSEN1 \\
\hline CDC25A & EGFR \\
\hline CREBBP & HOXB7 \\
\hline DLG3 & GRIK2 \\
\hline CTNNB1 & HDAC1 \\
\hline CFTR & CLCN3 \\
\hline IFNG & IFNGR2 \\
\hline MAPK1 & TP53 \\
\hline CAV1 & CSK \\
\hline CANX & LPA \\
\hline FLAD1 & TRIM23 \\
\hline CBL & UBE2D2 \\
\hline MPRIP & YWHAB \\
\hline NEDD9 & PTPN12 \\
\hline CBL & KRT18 \\
\hline MAPK8IP2 & TIAM1 \\
\hline TRAF3 & USP7 \\
\hline GOLGA2 & TMED2 \\
\hline CRMP1 & ZNF24 \\
\hline SFN & TSC2 \\
\hline MDM4 & YWHAH \\
\hline FKBP4 & NR3C1 \\
\hline CBX3 & HIST1H1E \\
\hline TAF5 & TAF6 \\
\hline GYPC & MPP1 \\
\hline BMP6 & BMPR2 \\
\hline ILF2 & PRKDC \\
\hline COPA & COPB2 \\
\hline TAF1 & TAF11 \\
\hline EGFR & VAV1 \\
\hline CDK10 & ETS2 \\
\hline PSMA3 & SNRPF \\
\hline BFSP1 & VIM \\
\hline CD8A & CD8A \\
\hline
\end{tabular}




\begin{tabular}{|c|c|}
\hline EGFR & HTT \\
\hline NFIC & RFX1 \\
\hline THY1 & THY1 \\
\hline DDB1 & LMO4 \\
\hline SP100 & SUMO1 \\
\hline GNB2L1 & PTN \\
\hline PTPRS & RAB35 \\
\hline PAK1 & RAF1 \\
\hline PCBP1 & UGP2 \\
\hline FYN & WAS \\
\hline RELA & TP53BP2 \\
\hline ERBB2 & ERBB4 \\
\hline$A R$ & KAT5 \\
\hline IKBKE & IRF3 \\
\hline KIF22 & SIAH1 \\
\hline EGFR & SRC \\
\hline ACTN2 & KCNA4 \\
\hline APOA2 & APOF \\
\hline AQP5 & MDFI \\
\hline MAPK3 & RPS6KA3 \\
\hline FOS & TAF1 \\
\hline CCDC85B & $\mathrm{EZH} 2$ \\
\hline ATP5A1 & YWHAB \\
\hline CCDC85B & LMO3 \\
\hline LSM1 & PSMB8 \\
\hline TLR1 & TLR4 \\
\hline FGF1 & FGFR3 \\
\hline CD9 & KIT \\
\hline STX1A & STXBP2 \\
\hline IL12A & IL12B \\
\hline KRT8 & PNN \\
\hline CD40 & XRCC5 \\
\hline NFIX & RFX1 \\
\hline KIAA0368 & PCM1 \\
\hline GCA & GCA \\
\hline NUP205 & NUP93 \\
\hline CALR & SLC2A1 \\
\hline NCOA6 & RXRA \\
\hline KPNB1 & PLCD1 \\
\hline SFN & TJP2 \\
\hline PTPRS & SETDB1 \\
\hline C1QBP & PRKCZ \\
\hline KRT14 & PKP1 \\
\hline FGF8 & FGFR3 \\
\hline PSEN2 & RAB11A \\
\hline KIF5A & TP53BP2 \\
\hline ESR1 & SHC1 \\
\hline
\end{tabular}




\begin{tabular}{|c|c|}
\hline ITK & KPNA2 \\
\hline BRCA1 & NFYA \\
\hline CCL21 & VCAN \\
\hline $\mathrm{CSH} 1$ & SMAD2 \\
\hline RET & STAT3 \\
\hline CAPN1 & CAST \\
\hline GNA15 & TTC1 \\
\hline HCFC1 & PDCD2 \\
\hline AR & CTNNB1 \\
\hline PCNA & XRCC5 \\
\hline CRYBB2 & HSPB1 \\
\hline AR & NCOA1 \\
\hline JUNB & SMAD3 \\
\hline MECP2 & SKI \\
\hline INPP5A & YWHAZ \\
\hline RPA4 & XPA \\
\hline LMNA & TMPO \\
\hline AKT1 & BAD \\
\hline TP53 & UBE2I \\
\hline STAT1 & STAT3 \\
\hline RANBP2 & UBE2I \\
\hline BAT2 & C1QBP \\
\hline DVL1 & USP13 \\
\hline MAGEA11 & WTAP \\
\hline CASP7 & HSPA5 \\
\hline BCAP31 & CASP1 \\
\hline LCK & NEDD9 \\
\hline BAD & BCL2L1 \\
\hline ACTN1 & COL17A1 \\
\hline CDK7 & E2F1 \\
\hline FEZ1 & PTPRS \\
\hline BRCA2 & КАТ2B \\
\hline CUL5 & SMAD2 \\
\hline PDLIM7 & WWP2 \\
\hline GLI2 & ZIC1 \\
\hline CDKN1A & TSG101 \\
\hline IQGAP1 & S100B \\
\hline EP300 & SMAD4 \\
\hline HNRNPM & JUN \\
\hline RELA & TAF6 \\
\hline EGFR & MAP4K1 \\
\hline HDAC1 & ZMYND11 \\
\hline DMPK & HSPB2 \\
\hline MAPK14 & MAPK3 \\
\hline HSPA2 & MEOX2 \\
\hline CCNB1 & TGFBR2 \\
\hline AHR & GTF2F2 \\
\hline
\end{tabular}




\begin{tabular}{|c|c|}
\hline PLAU & SERPINE1 \\
\hline CCT5 & IMMT \\
\hline COL3A1 & SPARC \\
\hline CSNK2A1 & PIN1 \\
\hline RNF4 & TBP \\
\hline SAFB & TAF15 \\
\hline$A B C C 8$ & KCNJ11 \\
\hline CBL & CRKL \\
\hline AVPR1A & GRK5 \\
\hline APBA2 & APP \\
\hline CTNNA1 & PSEN1 \\
\hline ADAR & HDLBP \\
\hline ARCN1 & COPB1 \\
\hline JUN & STAT4 \\
\hline $\mathrm{TSC} 1$ & $\mathrm{TSC} 2$ \\
\hline PKIA & PRKACA \\
\hline FCGR2A & LGALS3 \\
\hline COL5A1 & THBS1 \\
\hline HSPB3 & UNC119 \\
\hline FOS & TBP \\
\hline HMGB2 & POU2F2 \\
\hline SKI & SMAD3 \\
\hline CTNNB1 & PTPRJ \\
\hline AKAP9 & TUBGCP3 \\
\hline DDX17 & SF1 \\
\hline GRB2 & SYP \\
\hline NR3C1 & ZBTB16 \\
\hline $\mathrm{BCL} 2$ & BNIP3 \\
\hline HNRNPM & LMO3 \\
\hline ITGB2 & SYK \\
\hline CYP11A1 & FDX1 \\
\hline ACTN1 & ATXN2 \\
\hline AKAP9 & CLIC1 \\
\hline PHLDA1 & RPL14 \\
\hline ARF1 & PLD1 \\
\hline AKT1 & $A R$ \\
\hline CD3E & ZAP70 \\
\hline CD9 & ITGA3 \\
\hline CCND1 & КАТ2B \\
\hline PKN1 & VIM \\
\hline CCND3 & CDK4 \\
\hline ALOX12 & TRIB1 \\
\hline JUN & VDR \\
\hline PPP4C & PPP4R1 \\
\hline ICAM1 & ITGAM \\
\hline ETS2 & SRC \\
\hline F2 & SERPIND1 \\
\hline
\end{tabular}




\begin{tabular}{|c|c|}
\hline CEBPZ & NFYB \\
\hline CBL & PTPN11 \\
\hline F9 & LRP1 \\
\hline ATF2 & CSNK2A1 \\
\hline DAZ1 & DAZL \\
\hline CAV1 & SRC \\
\hline CDC25A & YWHAB \\
\hline PFN1 & WASF1 \\
\hline ABL1 & RAD9A \\
\hline RPS27A & SMAD2 \\
\hline FYN & KHDRBS1 \\
\hline CCL20 & CCR6 \\
\hline KRT81 & SKIL \\
\hline LOR & VIM \\
\hline CTLA4 & PPP2R5A \\
\hline CRK & PDGFRB \\
\hline HMOX2 & SETDB1 \\
\hline FGFR3 & GPSM3 \\
\hline HSPA1A & SLC5A1 \\
\hline PLK1 & PSMB1 \\
\hline FMOD & TGFB1 \\
\hline OPRL1 & PNOC \\
\hline BRCA1 & MLH1 \\
\hline PRKG1 & TNNT1 \\
\hline ABL1 & CREB1 \\
\hline KLF6 & SP1 \\
\hline CCNB1 & CCNF \\
\hline NFKB2 & SUMO1 \\
\hline CDK9 & HSPA1A \\
\hline ETS1 & UBE2I \\
\hline B2M & CD1D \\
\hline TK1 & TP53 \\
\hline CSNK1D & GJA1 \\
\hline SCNN1G & WWP2 \\
\hline JUN & RBM39 \\
\hline AXIN1 & CSNK1A1 \\
\hline NROB2 & PPARG \\
\hline SMAD1 & SMAD4 \\
\hline FOXO1 & SRC \\
\hline CD8A & HLA \\
\hline TUBB & YWHAB \\
\hline ARID3A & BTK \\
\hline IRS1 & PRKCD \\
\hline TGFB1 & TGFBR1 \\
\hline SNAP25 & TRIM9 \\
\hline LRP1 & PLAUR \\
\hline CANX & $\mathrm{TF}$ \\
\hline
\end{tabular}




\begin{tabular}{|c|c|}
\hline MSN & SPN \\
\hline ATXN1 & GAPDH \\
\hline F13A1 & FGA \\
\hline COPS5 & MDM2 \\
\hline CREBBP & RELA \\
\hline GHR & PLCG1 \\
\hline PTPN1 & TYK2 \\
\hline ADAM15 & ARHGEF7 \\
\hline NONO & SFPQ \\
\hline ELK4 & SRF \\
\hline GBP2 & PAFAH1B3 \\
\hline POU2AF1 & SIAH1 \\
\hline BARD1 & BRCA1 \\
\hline ELN & FBN2 \\
\hline RAPGEF1 & SHC1 \\
\hline IL6R & STAT3 \\
\hline POLR2A & POLR2C \\
\hline EFNA5 & EPHA7 \\
\hline BMI1 & ZBTB16 \\
\hline KIF1A & KIF1A \\
\hline RPA3 & RPA4 \\
\hline NCOA1 & TBP \\
\hline BRAF & SFN \\
\hline MCL1 & MCL1 \\
\hline EIF3I & EIF4G1 \\
\hline ACTN2 & TTN \\
\hline ERBB4 & PIK3R2 \\
\hline CASP8 & MAPK3 \\
\hline RFC2 & RFC4 \\
\hline PRSS1 & SERPINF2 \\
\hline HSP90AB1 & IKBKE \\
\hline EGFR & PDGFRB \\
\hline MAPK14 & MEF2C \\
\hline $\mathrm{CDC7}$ & MCM2 \\
\hline CREBBP & CSK \\
\hline MED1 & RARA \\
\hline MPHOSPH6 & TP53 \\
\hline $\mathrm{EZH} 2$ & GTF3C1 \\
\hline SNAP25 & STX3 \\
\hline BCL2L1 & FKBP8 \\
\hline FKBP8 & MAPK14 \\
\hline CANX & TSHR \\
\hline PLK3 & TP53 \\
\hline CTGF & VEGFA \\
\hline GOLGA2 & TRIM29 \\
\hline COPA & PDGFRB \\
\hline ESR1 & IGF1R \\
\hline
\end{tabular}




\begin{tabular}{|c|c|}
\hline BATF & IFI35 \\
\hline CD4 & UNC119 \\
\hline PIN1 & PKMYT1 \\
\hline DCT & DCT \\
\hline IGF2 & IGF2R \\
\hline COPS5 & S100A7 \\
\hline FKBP3 & MDM2 \\
\hline FBN1 & MFAP2 \\
\hline MAPK1 & PEBP1 \\
\hline GIT2 & PXN \\
\hline FGR & SLAMF1 \\
\hline YWHAE & YWHAH \\
\hline CCND1 & TSC2 \\
\hline C1QBP & MAPK1 \\
\hline CHML & RAB1A \\
\hline PI4KB & SFN \\
\hline CBX3 & CBX5 \\
\hline PARP1 & PCNA \\
\hline CTNNA1 & DLG1 \\
\hline SF1 & TRIM23 \\
\hline GAPDH & PSMD11 \\
\hline BRAF & RAF1 \\
\hline KRT8 & RAF1 \\
\hline$A X L$ & SRC \\
\hline YWHAE & YWHAZ \\
\hline EWSR1 & PTK2B \\
\hline EYA2 & GNAI2 \\
\hline CLIC1 & LSM1 \\
\hline CREBBP & MYB \\
\hline IL4R & PTPN6 \\
\hline CRKL & PIK3R2 \\
\hline MYOD1 & SMAD7 \\
\hline CANX & LRP1 \\
\hline APPBP2 & CREB3 \\
\hline HTR1B & HTR1D \\
\hline ATM & RAD51 \\
\hline HOXA1 & MDFI \\
\hline DAZ1 & DAZ1 \\
\hline HLA & TRA \\
\hline RPA2 & UNC119 \\
\hline PSMD1 & SMAD1 \\
\hline CALR & MBL2 \\
\hline NTRK2 & TRAF6 \\
\hline RB1 & RING1 \\
\hline EEF1A1 & PTPN4 \\
\hline ARNTL & CSNK1E \\
\hline BAT2 & MAN2C1 \\
\hline
\end{tabular}




\begin{tabular}{|c|c|}
\hline CBX3 & CBX3 \\
\hline BBC3 & $\mathrm{BCL} 2$ \\
\hline FTL & MAP3K12 \\
\hline FADD & FAS \\
\hline BTK & JAK1 \\
\hline BBC3 & BCL2L1 \\
\hline PKN1 & RHOA \\
\hline GRB2 & SYN1 \\
\hline MAPK3 & PTPN7 \\
\hline DHFR & MDM2 \\
\hline CRK & MAPK8 \\
\hline ETS1 & RUNX2 \\
\hline MLH1 & MYC \\
\hline PAFAH1B2 & SLA \\
\hline ID2 & TCF4 \\
\hline SP1 & SREBF1 \\
\hline HSPA5 & LCT \\
\hline ATP2B4 & DLG2 \\
\hline GTF2F1 & MSX2 \\
\hline PSMC2 & PSMD5 \\
\hline CUL4A & SKP2 \\
\hline NCOA1 & NKX2 \\
\hline PLAT & SERPINA5 \\
\hline ERBB2 & PTPN11 \\
\hline CRMP1 & PLA2G2A \\
\hline RIN1 & YWHAB \\
\hline EZR & SELL \\
\hline CTSA & NEU1 \\
\hline LYN & SYK \\
\hline CTNND1 & PTPN6 \\
\hline CRMP1 & EEF1D \\
\hline $\mathrm{BCL} 2$ & HSPA1A \\
\hline HTT & PFN2 \\
\hline MCM7 & NFKBIA \\
\hline $\mathrm{ABI} 2$ & $\mathrm{VCL}$ \\
\hline GTF2I & PRKG1 \\
\hline JAK1 & SHB \\
\hline CRHR1 & GNAI1 \\
\hline CST3 & CTSB \\
\hline SNAP25 & VAMP2 \\
\hline IMMT & STX5 \\
\hline PRKCE & SLC25A4 \\
\hline MDFI & SLC9A1 \\
\hline ARNT & GTF2F2 \\
\hline NME4 & NME4 \\
\hline NUMB & SIAH1 \\
\hline NEK2 & NEK2 \\
\hline
\end{tabular}




\begin{tabular}{|c|c|}
\hline RBL1 & TAF1 \\
\hline COPS6 & PHYHIP \\
\hline NFYA & NFYB \\
\hline GTF2I & STAT1 \\
\hline MAPK1 & TSC2 \\
\hline PTP4A2 & RABGGTB \\
\hline PTPRF & SKIL \\
\hline EPHA2 & SLA \\
\hline FXR2 & RBMX \\
\hline CCDC106 & EEF1G \\
\hline CCND3 & RARA \\
\hline CAV1 & NOS2 \\
\hline EXOSC2 & UPF1 \\
\hline HMGB2 & RAG1 \\
\hline COL3A1 & MAG \\
\hline RARB & RXRG \\
\hline HDAC2 & RFX5 \\
\hline NRIP1 & PPARG \\
\hline PRPS1 & PRPSAP1 \\
\hline ATF6 & ATF6 \\
\hline SETDB1 & SUMO2 \\
\hline NFYA & TP53 \\
\hline CALM1 & SYT1 \\
\hline HRAS & RIN1 \\
\hline ACTN1 & CTNNA1 \\
\hline LMNA & RB1 \\
\hline SREBF1 & YY1 \\
\hline CSNK2A1 & DVL1 \\
\hline ANP32A & KPNA5 \\
\hline CCDC130 & ZNF24 \\
\hline INPP4A & SMAD1 \\
\hline IL6ST & TYK2 \\
\hline CAV1 & FLOT2 \\
\hline ADORA2B & EZR \\
\hline GRB2 & TNFRSF1A \\
\hline NCF2 & RAC1 \\
\hline ID1 & TCF12 \\
\hline ANP32A & XPO1 \\
\hline FLNA & TRIO \\
\hline КАТ2B & ONECUT1 \\
\hline GRB2 & KRT18 \\
\hline GZMB & SRGN \\
\hline NFKB1 & NKRF \\
\hline ACTN2 & KCNA5 \\
\hline ACTA1 & PFN1 \\
\hline CXCL9 & DPP4 \\
\hline MS4A2 & SYK \\
\hline
\end{tabular}




\begin{tabular}{|c|c|}
\hline ACAN & TNR \\
\hline SRPR & SSR2 \\
\hline PDGFRB & SRC \\
\hline SUMO1 & UBE2I \\
\hline FNTA & TGFBR1 \\
\hline FABP1 & PPARG \\
\hline EGFR & TJP1 \\
\hline BTK & WAS \\
\hline CD28 & PIK3R1 \\
\hline PEA15 & RPS6KA3 \\
\hline PSAP & PSAP \\
\hline CD7 & SECTM1 \\
\hline LASP1 & MDFI \\
\hline FLI1 & SRF \\
\hline GNB1 & KCNJ3 \\
\hline GAS6 & TYRO3 \\
\hline PTK2B & PTK2B \\
\hline PRKCZ & RELA \\
\hline MAX & MXI1 \\
\hline EPHB1 & NCK1 \\
\hline F11 & KNG1 \\
\hline SELP & SNX17 \\
\hline CBL & SYK \\
\hline COIL & PSME3 \\
\hline SYT5 & SYT5 \\
\hline MAPK3 & MYC \\
\hline BRCA1 & RBBP4 \\
\hline ATF3 & JUN \\
\hline IRF8 & TRAF6 \\
\hline FEZ1 & STAR \\
\hline CSNK1A1 & MDM4 \\
\hline KLF4 & KLF6 \\
\hline ADD1 & SPTB \\
\hline KRT1 & YWHAQ \\
\hline AFAP1 & PRKCE \\
\hline CRMP1 & PAFAH1B3 \\
\hline HIF1A & STAT3 \\
\hline NDN & NGFR \\
\hline MAPRE3 & RAB1A \\
\hline ITPR1 & ITPR3 \\
\hline FXR1 & FXR1 \\
\hline GCA & SRI \\
\hline ERG & JUN \\
\hline CRKL & PXN \\
\hline HSP90AB1 & МАРЗКЗ \\
\hline GRIA2 & SPTAN1 \\
\hline FYN & MS4A1 \\
\hline
\end{tabular}




\begin{tabular}{|c|c|}
\hline MYC & NFYC \\
\hline ITGB3 & ITGB3BP \\
\hline КАТ2B & TCF3 \\
\hline COPS6 & cox17 \\
\hline DRAP1 & TBP \\
\hline NR1H2 & RXRB \\
\hline CTNNB1 & FHL2 \\
\hline GNAI3 & RGS7 \\
\hline GJB1 & OCLN \\
\hline RAF1 & RAP1A \\
\hline HDAC1 & SP3 \\
\hline BAT3 & SETDB1 \\
\hline SETDB1 & VIM \\
\hline ERCC3 & GTF2H1 \\
\hline AMD1 & AMD1 \\
\hline APOA1 & PCMT1 \\
\hline TNFRSF8 & TNFSF8 \\
\hline $\mathrm{FGB}$ & FGG \\
\hline EP300 & TSG101 \\
\hline HLA & UNC119 \\
\hline ANK1 & RHAG \\
\hline RASA1 & SRC \\
\hline PHB & TP53 \\
\hline BNIP3 & CD47 \\
\hline HMGB1 & POU5F1 \\
\hline GABRA1 & PRKCD \\
\hline CD40 & XRCC6 \\
\hline FN1 & TNC \\
\hline CTTN & MYLK \\
\hline CHN1 & GTF3C1 \\
\hline GRIN2A & PTK2B \\
\hline SMAD5 & SOX5 \\
\hline HDAC1 & RBBP4 \\
\hline ACTN2 & DLG1 \\
\hline CALM1 & CNN1 \\
\hline IL16 & KCNJ4 \\
\hline SF1 & TAF15 \\
\hline COL1A1 & SPARC \\
\hline TP53 & WT1 \\
\hline TRIM21 & YWHAZ \\
\hline GSN & VDAC1 \\
\hline SFN & WEE1 \\
\hline TRIM25 & TRIM25 \\
\hline ITGA5 & SPP1 \\
\hline NCK1 & TNK2 \\
\hline POLR2B & POLR2L \\
\hline IL4R & JAK1 \\
\hline
\end{tabular}




\begin{tabular}{|c|c|}
\hline CDC16 & SMAD2 \\
\hline EP300 & PPARD \\
\hline TFE3 & TFE3 \\
\hline S100A11 & S100A11 \\
\hline MAP2K5 & PRKCZ \\
\hline ETS1 & JUN \\
\hline ERCC5 & PCNA \\
\hline PAFAH1B3 & TP53 \\
\hline GRB2 & SYK \\
\hline ARID3A & E2F1 \\
\hline CCDC85B & KRT6A \\
\hline ARFIP2 & RAC1 \\
\hline ANXA1 & EGFR \\
\hline AKAP13 & GNAQ \\
\hline CBL & CD5 \\
\hline ACTG1 & DSTN \\
\hline CREBBP & DDX5 \\
\hline SMAD2 & SNW1 \\
\hline TRAF6 & USP7 \\
\hline RB1 & SNAPC3 \\
\hline MVK & MVK \\
\hline KIF23 & USP8 \\
\hline PRKD1 & YWHAQ \\
\hline DAZAP2 & 2-Sep \\
\hline BIN1 & CUX1 \\
\hline PABPC4 & PHLDA1 \\
\hline CCDC85B & ZNF165 \\
\hline ATM & TP53BP1 \\
\hline HDAC1 & MAD1L1 \\
\hline EIF2B2 & NCK1 \\
\hline HTT & RASA1 \\
\hline DR1 & NFIL3 \\
\hline ZNF174 & ZNF24 \\
\hline CDKN1A & SKP2 \\
\hline SH3GL3 & SNRPN \\
\hline CENPF & CENPF \\
\hline RAB5A & RIN1 \\
\hline FLI1 & KLF1 \\
\hline COPS6 & MYCBP \\
\hline SYK & TUBA1A \\
\hline ADAM15 & LCK \\
\hline VASP & WWP2 \\
\hline VAV1 & ZAP70 \\
\hline LPL & LRP2 \\
\hline COPS6 & RPA2 \\
\hline DAG1 & FYN \\
\hline PRNP & PSMA3 \\
\hline
\end{tabular}




\begin{tabular}{|c|c|}
\hline LTBR & TRAF3 \\
\hline CSNK2A1 & HNRNPA2B1 \\
\hline PFN2 & PLAUR \\
\hline NR3C1 & TSG101 \\
\hline MAP2K3 & MAPK14 \\
\hline ESR1 & MNAT1 \\
\hline ALDH2 & HSPD1 \\
\hline ATF3 & FGFR3 \\
\hline DYNLL1 & NTRK2 \\
\hline JUN & TBP \\
\hline INPP5D & SHC1 \\
\hline EFNA1 & EPHA3 \\
\hline BMP2 & BMPR2 \\
\hline DLGAP1 & DYNLL1 \\
\hline HDAC1 & MYOD1 \\
\hline EIF4G1 & PDCD4 \\
\hline TXN & TXNIP \\
\hline CANX & SLC2A1 \\
\hline DLAT & PDHB \\
\hline DCTN1 & PAFAH1B1 \\
\hline BMP2 & BMPR1A \\
\hline ALK & JAK3 \\
\hline EZR & SELP \\
\hline MAX & MXD1 \\
\hline KPNA2 & NFKBIB \\
\hline COL4A2 & COL4A2 \\
\hline $\mathrm{BCR}$ & CRKL \\
\hline KCNA4 & SAT1 \\
\hline CRK & XPO1 \\
\hline GDI2 & RAB11A \\
\hline IGF2 & IGFBP4 \\
\hline FGF1 & SYT1 \\
\hline IL16 & KCNJ10 \\
\hline ALK & PTN \\
\hline NCOA2 & PRMT1 \\
\hline ACTN1 & CDK5R1 \\
\hline CRK & EPHB3 \\
\hline C19orf57 & GPS2 \\
\hline ATN1 & TLE1 \\
\hline PTOV1 & SPTAN1 \\
\hline SMAD7 & XRCC6 \\
\hline SFRS5 & TNK2 \\
\hline ACVR1B & INHBB \\
\hline CDK2 & UBE2A \\
\hline FEZ1 & PRKCZ \\
\hline PDGFRA & PDGFRB \\
\hline CLTC & VCL \\
\hline
\end{tabular}




\begin{tabular}{|c|c|}
\hline DAG1 & DRP2 \\
\hline NRIP1 & PRMT1 \\
\hline HLA & HSPA5 \\
\hline PTGES3 & TERT \\
\hline CDK2 & EP300 \\
\hline IDUA & MPP3 \\
\hline IFI16 & TP53 \\
\hline TF & TUBB3 \\
\hline RNPS1 & SFRS6 \\
\hline APP & NAE1 \\
\hline HDAC2 & YY1 \\
\hline ABL1 & RB1 \\
\hline CTSL1 & SERPINB3 \\
\hline C19orf57 & RUNX1T1 \\
\hline CSNK2A1 & PSMA4 \\
\hline ZNF165 & ZNF250 \\
\hline ELF1 & NFKB1 \\
\hline CREBBP & HIF1A \\
\hline BMPR1A & BMPR1A \\
\hline CAPNS1 & IL2RG \\
\hline NMI & PSMA1 \\
\hline EGFR & STAT5A \\
\hline POU2F2 & XRCC6 \\
\hline TAF11 & TAF4 \\
\hline CNTN2 & L1CAM \\
\hline ALK & IRS1 \\
\hline DSC3 & JUP \\
\hline ARF1 & COPB1 \\
\hline AKAP9 & TRIP10 \\
\hline CCND1 & RB1 \\
\hline LRP1 & SKIL \\
\hline GTF2A2 & TBP \\
\hline HMGA1 & SP1 \\
\hline ARL3 & UNC119 \\
\hline HLA & TAP1 \\
\hline ATN1 & HSPG2 \\
\hline GRB2 & MUC1 \\
\hline FYN & GNB2L1 \\
\hline RIPK1 & TRAF5 \\
\hline GRB2 & MST1R \\
\hline AREG & EGFR \\
\hline MMP9 & THBS2 \\
\hline NMI & STAT1 \\
\hline CRKL & KIT \\
\hline ARR3 & PTAFR \\
\hline PLCG1 & SH3BP2 \\
\hline JUN & TCF20 \\
\hline
\end{tabular}




\begin{tabular}{|c|c|}
\hline ETF1 & GSPT1 \\
\hline TRIM31 & TRIM31 \\
\hline CRKL & SHC1 \\
\hline $\mathrm{CBL}$ & LCP2 \\
\hline FABP4 & VIM \\
\hline BCL2 & ITPR1 \\
\hline SFN & ST5 \\
\hline DDB1 & ERCC8 \\
\hline ССТЗ & EIF2B2 \\
\hline C7 & CLU \\
\hline NGFR & SHC1 \\
\hline HSPA1A & RHOA \\
\hline MST1R & SHC1 \\
\hline ABCD3 & PEX19 \\
\hline BRF1 & RBL1 \\
\hline CDC6 & ORC1L \\
\hline $\mathrm{AUH}$ & AUH \\
\hline CAV1 & TGFBR1 \\
\hline GTF2F1 & PTN \\
\hline ATN1 & ECM1 \\
\hline CD40 & TRAF5 \\
\hline CTNND1 & SRC \\
\hline FGF1 & FGFBP1 \\
\hline GRB10 & IGF1R \\
\hline NGFR & TRAF6 \\
\hline IQGAP2 & RAC1 \\
\hline BAT1 & DNM2 \\
\hline RNPS1 & SART3 \\
\hline COIL & CSNK2B \\
\hline F10 & F7 \\
\hline CACNA1C & RYR2 \\
\hline MSH2 & PCNA \\
\hline ATXN1 & YWHAE \\
\hline KAT5 & MYC \\
\hline RELB & SMARCB1 \\
\hline PPP2CA & PPP2R5E \\
\hline EWSR1 & TSPAN3 \\
\hline CCNO & PCNA \\
\hline CRYBA4 & CRYBB1 \\
\hline CDK2 & TP53 \\
\hline ERBB2 & PIK3R2 \\
\hline BNIP2 & CREB3 \\
\hline APP & GSN \\
\hline ARAF & MAP2K2 \\
\hline POLB & XRCC1 \\
\hline $\mathrm{SHC1}$ & STAT5A \\
\hline MAX & TEAD1 \\
\hline
\end{tabular}




\begin{tabular}{|c|c|}
\hline SNAP25 & STX1A \\
\hline ATF6 & SRF \\
\hline GTF2I & SRF \\
\hline FOS & RUNX1 \\
\hline ORM1 & SERPINE1 \\
\hline DAB2 & FGR \\
\hline APC & KIFAP3 \\
\hline DLG1 & ERBB4 \\
\hline CRKL & DOCK2 \\
\hline DLAT & PDK1 \\
\hline MAPK7 & YWHAB \\
\hline HGF & SDC1 \\
\hline ARHGEF7 & PPM1F \\
\hline HDAC1 & HDAC2 \\
\hline PIK3CD & PIK3R2 \\
\hline ADORA1 & GNAI2 \\
\hline BCL7A & RELB \\
\hline BAT3 & CTSE \\
\hline TAF10 & TAF4 \\
\hline CBX1 & UCHL1 \\
\hline CDC6 & CDKN2A \\
\hline ID3 & MYOD1 \\
\hline NSMAF & TNFRSF1A \\
\hline RRAD & YWHAZ \\
\hline CDK5 & MAPT \\
\hline DFFA & EWSR1 \\
\hline CTNNB1 & NCOA2 \\
\hline PIK3R1 & PTPN11 \\
\hline PIK3CA & PIK3R1 \\
\hline LHX2 & MSX1 \\
\hline МАРЗКЗ & YWHAB \\
\hline HSP90AA1 & МАРЗКЗ \\
\hline ESR2 & STAT5A \\
\hline IL16 & KCNJ2 \\
\hline PTN & SSR1 \\
\hline CREBBP & GCM1 \\
\hline MAP2K3 & SMAD7 \\
\hline LCN2 & MMP2 \\
\hline BAD & YWHAZ \\
\hline EZR & VCAM1 \\
\hline USF1 & USF2 \\
\hline DLG2 & ERBB4 \\
\hline CBL & CRK \\
\hline MARK3 & YWHAB \\
\hline EDNRB & GNA11 \\
\hline DAZAP2 & NDUFA5 \\
\hline FBLN1 & SKIL \\
\hline
\end{tabular}




\begin{tabular}{|c|c|}
\hline IGF1R & MDM2 \\
\hline BAD & YWHAQ \\
\hline HLA & KIR2DL1 \\
\hline BMP4 & BMPR2 \\
\hline KNG1 & VTN \\
\hline CSNK2B & NOLC1 \\
\hline GSN & PTK2B \\
\hline NEFH & PKN1 \\
\hline SYK & VAV1 \\
\hline GAP43 & PLCD1 \\
\hline MYOD1 & RXRA \\
\hline RPGR & TUBG1 \\
\hline PCNA & POLD3 \\
\hline ITGB2 & PTK2B \\
\hline CYTH1 & ITGB2 \\
\hline DLG3 & KCNJ12 \\
\hline CSF3R & LYN \\
\hline FXR1 & GBP2 \\
\hline$A X L$ & PIK3R1 \\
\hline CXCL12 & FN1 \\
\hline EIF3I & TGFB1 \\
\hline DLGAP1 & GRIN2A \\
\hline INSR & SHC1 \\
\hline ACVR1 & GDF5 \\
\hline LCP2 & PTPN6 \\
\hline CTNNB1 & PTPRF \\
\hline BCL2A1 & BIK \\
\hline DYRK1A & YWHAE \\
\hline ESR1 & $\mathrm{NR} 2 \mathrm{C} 1$ \\
\hline HSF1 & SYMPK \\
\hline APPBP2 & TBL3 \\
\hline ANG & TDGF1 \\
\hline HDAC1 & STAT3 \\
\hline CYCS & HSPB1 \\
\hline BTK & LYN \\
\hline AIP & HSP90AA1 \\
\hline $\mathrm{A} 2 \mathrm{M}$ & IL10 \\
\hline ADH5 & ADH5 \\
\hline PLK1 & PSMB6 \\
\hline EGFR & SH3GL2 \\
\hline RBBP6 & RPS14 \\
\hline HOXB1 & PAX6 \\
\hline CAV1 & PTEN \\
\hline KCNJ6 & KCNJ9 \\
\hline ALK & SHC1 \\
\hline HSP90AA1 & NPAS2 \\
\hline ERCC3 & PSMC5 \\
\hline
\end{tabular}




\begin{tabular}{|c|c|}
\hline GRIN1 & GRIN2A \\
\hline ACVR2B & BMP7 \\
\hline FLNA & PSEN2 \\
\hline FOSL2 & JUN \\
\hline SNRPA1 & SNRPB2 \\
\hline B2M & HFE \\
\hline KRT15 & TSG101 \\
\hline CAMK2G & RRAD \\
\hline CLTC & XRCC6 \\
\hline ESR1 & SMARCA2 \\
\hline KRT15 & MAD2L1BP \\
\hline COPB1 & HLA \\
\hline CAV1 & PTPN1 \\
\hline EPHB3 & RYK \\
\hline POU3F2 & POU3F4 \\
\hline ATF2 & MAPK11 \\
\hline ABLIM1 & SFN \\
\hline CELSR2 & GARS \\
\hline APBB1 & LRP1 \\
\hline CUL1 & MYC \\
\hline HNF1A & PCBD1 \\
\hline SNAP23 & VAMP2 \\
\hline MAX & SMAD3 \\
\hline TSC22D3 & TSC22D3 \\
\hline TAF10 & TAF12 \\
\hline CHGB & FGFR3 \\
\hline $\mathrm{CHM}$ & RAB7A \\
\hline CSNK2B & LYST \\
\hline CKS1B & SKP2 \\
\hline F8A1 & $\mathrm{HTT}$ \\
\hline EP300 & HNF1A \\
\hline COIL & SNRPB \\
\hline IL6ST & TLE1 \\
\hline ARR3 & CAPNS1 \\
\hline CNR1 & GNAI2 \\
\hline GSTA1 & GSTA1 \\
\hline BCL3 & TBP \\
\hline ATP5A1 & YWHAZ \\
\hline CD44 & COL14A1 \\
\hline CDC34 & CSNK2A1 \\
\hline $\mathrm{CDH} 1$ & CSE1L \\
\hline TGM2 & TGM2 \\
\hline CEBPA & MYC \\
\hline C5 & C6 \\
\hline ALPP & PIGK \\
\hline MDM4 & SMAD3 \\
\hline EPB41 & SPTAN1 \\
\hline
\end{tabular}




\begin{tabular}{|c|c|}
\hline NR3C1 & SMARCD1 \\
\hline ARAF & EFEMP1 \\
\hline DLG3 & GRIN2B \\
\hline BAK1 & TP53 \\
\hline CRKL & SYK \\
\hline ARSE & NDN \\
\hline TOP2B & TP53 \\
\hline PSMD4 & RAD23A \\
\hline CDC7 & MCM5 \\
\hline CALM1 & IQGAP1 \\
\hline NCOA6 & XRCC5 \\
\hline COL2A1 & THBS1 \\
\hline AKT1 & IMPDH2 \\
\hline COPB2 & NFKB1 \\
\hline FGR & HCLS1 \\
\hline NFKBIE & RELA \\
\hline CYP1A2 & POR \\
\hline NCOR2 & NR4A1 \\
\hline KPNA2 & SLC2A2 \\
\hline APOB & LIPC \\
\hline CREM & TBP \\
\hline NF2 & SPTBN1 \\
\hline CTNNB1 & IQGAP1 \\
\hline BMI1 & MLL \\
\hline CR2 & IFITM1 \\
\hline CTNNB1 & EZR \\
\hline GTF2B & GTF2E1 \\
\hline FBLN1 & SMAD4 \\
\hline BRF1 & RBL2 \\
\hline PCNA & RFC4 \\
\hline CD27 & TRAF5 \\
\hline ORC2L & TERF2 \\
\hline KIT & PTPN11 \\
\hline FOS & NCOR2 \\
\hline SKI & SMAD4 \\
\hline EP300 & GPS2 \\
\hline ABL1 & INPPL1 \\
\hline ITK & PLCG1 \\
\hline KIFAP3 & SRC \\
\hline BMPR2 & LIMK1 \\
\hline GTF2E2 & GTF2F1 \\
\hline LMNA & ZNF239 \\
\hline EEF1G & EFNA1 \\
\hline AXIN1 & AXIN1 \\
\hline BCL2 & PIN1 \\
\hline RBBP4 & XRCC6 \\
\hline MAG & MAP1B \\
\hline
\end{tabular}




\begin{tabular}{|c|c|}
\hline PPP4C & TRAF6 \\
\hline ARFIP2 & ARFIP2 \\
\hline APBB1 & VASP \\
\hline LSM1 & PSMB5 \\
\hline BRCA1 & HDAC2 \\
\hline RPS6KB1 & TRAF4 \\
\hline NR3C1 & SMARCA2 \\
\hline CDC16 & CDC20 \\
\hline LASP1 & PLSCR1 \\
\hline WEE1 & YWHAB \\
\hline CDC34 & CSNK2B \\
\hline PLCG1 & TUB \\
\hline BRCA1 & CSTF1 \\
\hline CEBPA & MSX2 \\
\hline GDF15 & GDF15 \\
\hline APP & FBLN1 \\
\hline ACVR1B & TDGF1 \\
\hline COL1A1 & ITGA2 \\
\hline CSF2RB & MAD2L1 \\
\hline PZP & TGFB2 \\
\hline LMAN1 & MCFD2 \\
\hline EZR & EZR \\
\hline ADORA1 & DRD1 \\
\hline CASP8 & PEA15 \\
\hline FDPS & FGFR1 \\
\hline POP1 & RPP38 \\
\hline HSP90AA1 & RAF1 \\
\hline MAF & MYB \\
\hline CTNNA1 & VCL \\
\hline CSF3R & PTPN11 \\
\hline FCGR2B & PTPN6 \\
\hline CREBBP & E2F1 \\
\hline ADRBK1 & CAV1 \\
\hline PRKCD & SRC \\
\hline HSPA5 & VWF \\
\hline CDKN1A & RAB1A \\
\hline DLG4 & KCNJ12 \\
\hline AKAP9 & CSNK1E \\
\hline SMAD4 & TCTA \\
\hline KPNA1 & RAG1 \\
\hline EEF1D & VARS \\
\hline CREBBP & MYBL1 \\
\hline SELENBP1 & TRIP13 \\
\hline SOD1 & SOD1 \\
\hline HNRNPF & NCBP1 \\
\hline DDR2 & SRC \\
\hline CASP1 & IL18 \\
\hline
\end{tabular}




\begin{tabular}{|c|c|}
\hline ATXN1 & HIVEP1 \\
\hline GLI3 & ZIC1 \\
\hline HLA & TLE1 \\
\hline BMI1 & RNF2 \\
\hline JUN & NR3C1 \\
\hline BTK & KHDRBS1 \\
\hline DYNLL1 & NRF1 \\
\hline MCF2 & RHOA \\
\hline CLNS1A & PRMT5 \\
\hline GZMB & SERPINB9 \\
\hline RPA1 & TCEA2 \\
\hline CCKBR & PTPN11 \\
\hline PRMT1 & TP53 \\
\hline NFKB2 & PSMD11 \\
\hline NPR1 & PRKG1 \\
\hline CREB5 & JUN \\
\hline WEE1 & YWHAZ \\
\hline TYK2 & VAV1 \\
\hline MRPS12 & UNC119 \\
\hline DRAP1 & PIK3R3 \\
\hline GNA13 & PRKCD \\
\hline ITGA5 & L1CAM \\
\hline POLR2H & POLR2L \\
\hline SCARB2 & THBS1 \\
\hline BAT2 & PSMA3 \\
\hline FLNA & SMAD3 \\
\hline DLG3 & GRIN2A \\
\hline CES1 & GUSB \\
\hline HNRNPK & ITK \\
\hline FXR2 & RPIA \\
\hline CCNE1 & RBL1 \\
\hline EIF4B & PABPC1 \\
\hline CDC37 & HSP90AA1 \\
\hline SERPINB9 & SETDB1 \\
\hline NME1 & NME3 \\
\hline GAB1 & PLCG1 \\
\hline GRB2 & PRKAR1A \\
\hline CDK4 & CDKN2D \\
\hline IRAK1 & IRAK1 \\
\hline DHFR & FKBP1A \\
\hline PRMT5 & SNRPD3 \\
\hline TNFRSF9 & TRAF3 \\
\hline CSNK2A1 & PTPN1 \\
\hline MDH1 & MPP3 \\
\hline KCNK1 & KCNK1 \\
\hline ANXA6 & CR2 \\
\hline ETV6 & ETV6 \\
\hline
\end{tabular}




\begin{tabular}{|c|c|}
\hline NFKB1 & RELA \\
\hline HRAS & NF1 \\
\hline SFN & TBL3 \\
\hline CD19 & CD22 \\
\hline MAPT & S100B \\
\hline NEDD8 & PARP1 \\
\hline EPB42 & KRAS \\
\hline FN1 & IGFBP3 \\
\hline PLCG1 & TNK1 \\
\hline FNTA & TGFB1 \\
\hline LCK & NR3C1 \\
\hline EEF1G & PTPRS \\
\hline KIT & PIK3R2 \\
\hline AKT1 & PRKCZ \\
\hline PML & PML \\
\hline CSF2RB & IL3 \\
\hline EWSR1 & MDFI \\
\hline BCL3 & GTF2B \\
\hline ADRBK1 & SRC \\
\hline HNRNPA2B1 & HNRNPL \\
\hline CREBBP & MYBL2 \\
\hline CRMP1 & DDX18 \\
\hline ATN1 & PSME3 \\
\hline ATXN1 & USP7 \\
\hline APOA1 & TOMM20 \\
\hline DLG2 & DLGAP1 \\
\hline DDX5 & NCOA1 \\
\hline FADD & PRKCZ \\
\hline CASP1 & CASP1 \\
\hline GIT2 & PAK1 \\
\hline CDC25B & KAT2B \\
\hline $\mathrm{CDH} 2$ & $\mathrm{CDH} 4$ \\
\hline SRF & SSRP1 \\
\hline ACTG1 & CAP1 \\
\hline IPO5 & NUP214 \\
\hline GTF2F1 & SRF \\
\hline MCM3 & SSRP1 \\
\hline CALR & PRF1 \\
\hline HSPA1A & MSR1 \\
\hline GDI2 & RAB5A \\
\hline RAD51 & VIM \\
\hline VDR & VDR \\
\hline MSN & SELL \\
\hline LAMA4 & PTN \\
\hline FLT4 & GRB2 \\
\hline IL5 & IL5 \\
\hline APP & SNCB \\
\hline
\end{tabular}




\begin{tabular}{|c|c|}
\hline HSF2 & NUP62 \\
\hline H2AFX & SMARCA4 \\
\hline CDK5 & CDK5R1 \\
\hline COL1A1 & THBS1 \\
\hline HNF1A & SRC \\
\hline TNFRSF1B & TNFRSF1B \\
\hline JUND & SMAD3 \\
\hline FYN & GRIN2B \\
\hline BIRC3 & TRAF1 \\
\hline AP2B1 & TGFBR1 \\
\hline ACTN1 & PDLIM1 \\
\hline PIK3R1 & TGFBR2 \\
\hline S100A8 & S100A9 \\
\hline NEDD4 & SCNN1G \\
\hline CDC25B & ESR1 \\
\hline TFF1 & TFF1 \\
\hline NR3C1 & PTGES3 \\
\hline COIL & SMN1 \\
\hline KPNB1 & PTN \\
\hline GFI1 & RUNX1T1 \\
\hline DYRK1A & SFN \\
\hline COPS6 & VIM \\
\hline EBNA1BP2 & FGF3 \\
\hline TNFRSF14 & TRAF3 \\
\hline ATXN1 & CRK \\
\hline SON & YWHAB \\
\hline POMC & VTN \\
\hline FGR & SYK \\
\hline TEP1 & TP53 \\
\hline NCK1 & PDGFRB \\
\hline MCM2 & ORC1L \\
\hline ARHGEF7 & TUBB3 \\
\hline ARAF & SFN \\
\hline UCP3 & YWHAQ \\
\hline ARNT & HIF1A \\
\hline NTRK2 & PTPN1 \\
\hline GTF2B & GTF2F2 \\
\hline SMN1 & UNC119 \\
\hline ARNT & PTGES3 \\
\hline $\mathrm{CBL}$ & PLCG1 \\
\hline CREBBP & HNF1A \\
\hline NPPC & NPR3 \\
\hline GRB2 & KHDRBS1 \\
\hline NQO1 & NQO1 \\
\hline DLG4 & DLG4 \\
\hline RGS3 & YWHAZ \\
\hline CRMP1 & HNRNPH1 \\
\hline
\end{tabular}




\begin{tabular}{|c|c|}
\hline TRAF1 & USP7 \\
\hline MAP2K6 & SMAD7 \\
\hline NFKBIB & REL \\
\hline COPS6 & S100A10 \\
\hline HERPUD1 & PSEN1 \\
\hline CLTC & HIP1 \\
\hline ADRB2 & SRC \\
\hline BRAF & RAP1A \\
\hline CSNK1A1 & YWHAQ \\
\hline BCL2L1 & PSEN2 \\
\hline CACNA1A & SYT1 \\
\hline MST1R & PLCG1 \\
\hline CRY1 & MDFI \\
\hline EED & ITGA4 \\
\hline KIF5B & YWHAB \\
\hline EGFR & SNX1 \\
\hline $\mathrm{CDH} 2$ & JUP \\
\hline POLR2A & ZNF74 \\
\hline ACTN2 & ACTN3 \\
\hline HSPA1A & ST13 \\
\hline МАР3К14 & NFKB2 \\
\hline CALM1 & SCTR \\
\hline ICAM5 & PSEN2 \\
\hline CREB1 & SMARCA4 \\
\hline GNAI1 & IGF1R \\
\hline DAB2 & DVL3 \\
\hline IRF1 & КАТ2B \\
\hline HDAC2 & STAT3 \\
\hline LEPR & LEPR \\
\hline $\mathrm{CDC7}$ & МСM3 \\
\hline KIF5B & SNAP23 \\
\hline IFIT2 & IFIT3 \\
\hline $\mathrm{BCL} 2$ & CASP8 \\
\hline EIF4G1 & NCBP1 \\
\hline APBB1 & ATXN1 \\
\hline SUMO2 & UBE2I \\
\hline EEF1G & RGL2 \\
\hline IRS1 & MAPK8 \\
\hline KRT18 & PNN \\
\hline FYN & NR3C1 \\
\hline CHRM3 & CSNK1A1 \\
\hline GTF2E2 & XPA \\
\hline COL17A1 & KRT18 \\
\hline CA2 & SLC4A1 \\
\hline HCLS1 & MAP4K1 \\
\hline ANKRD7 & MARK3 \\
\hline ALDH2 & UNC119 \\
\hline
\end{tabular}




\begin{tabular}{|c|c|}
\hline CDK2 & ORC1L \\
\hline ARNT & NCOA2 \\
\hline JUP & PTPN14 \\
\hline GSPT1 & PABPC1 \\
\hline GATA1 & ZBTB16 \\
\hline CD44 & HBEGF \\
\hline ESR1 & SP3 \\
\hline CCNE1 & SKP2 \\
\hline NFKBIA & RELA \\
\hline GOLGA2 & RAB2A \\
\hline COL2A1 & PRELP \\
\hline S100A4 & UNC119 \\
\hline MAPK8 & REL \\
\hline NCOR2 & SNW1 \\
\hline CTSL1 & RXRA \\
\hline $\mathrm{AHR}$ & NEDD8 \\
\hline ASMTL & TDO2 \\
\hline GRN & HK3 \\
\hline GTF2F2 & TBP \\
\hline APEX1 & MUTYH \\
\hline APC & AXIN1 \\
\hline BAT3 & $\mathrm{CHN} 2$ \\
\hline MCM4 & MCM6 \\
\hline DRD4 & GRB2 \\
\hline МАР3К10 & YWHAE \\
\hline CBX5 & SMARCA4 \\
\hline ATP1A1 & TPT1 \\
\hline COPS6 & TDGF1 \\
\hline PDGFRB & SHC1 \\
\hline TRAF5 & USP7 \\
\hline ATRX & DAXX \\
\hline CSK & G3BP1 \\
\hline ALOX12 & LMNA \\
\hline PLCG1 & SHC1 \\
\hline NRAS & RAF1 \\
\hline LCK & PTK2B \\
\hline CRK & EGFR \\
\hline LTA & LTB \\
\hline RB1 & USP4 \\
\hline RPIA & RPIA \\
\hline GNB2L1 & GRIN2B \\
\hline RIPK1 & TRADD \\
\hline ITGB3BP & ITGB3BP \\
\hline SMAD2 & SNRNP70 \\
\hline CDK2 & МСM3 \\
\hline HOXA9 & PBX2 \\
\hline CLTC & PICALM \\
\hline
\end{tabular}




\begin{tabular}{|c|c|}
\hline TANK & TRAF1 \\
\hline NR3C1 & TBP \\
\hline ARF4 & EGFR \\
\hline DDX21 & JUN \\
\hline COPS6 & ZNF24 \\
\hline MDM2 & RPL11 \\
\hline MAGEA11 & PRDX3 \\
\hline EPB41 & SMAD3 \\
\hline FES & PIK3R1 \\
\hline ATXN1 & YWHAZ \\
\hline NCOA6 & PARP1 \\
\hline PTPN6 & SSTR2 \\
\hline RAC1 & RALBP1 \\
\hline ATXN1 & CIRBP \\
\hline EWSR1 & VPS72 \\
\hline JUN & NFE2L2 \\
\hline MAPT & SNCA \\
\hline ABL2 & CAT \\
\hline PSMB3 & PSMB5 \\
\hline МАРЗК8 & NFKB2 \\
\hline STAT3 & TSHR \\
\hline RUNX1T1 & ZBTB16 \\
\hline PEG3 & SIAH1 \\
\hline DLG1 & KCNA4 \\
\hline FN1 & MYOC \\
\hline NUP88 & NUP98 \\
\hline MAРК14 & МАРКАРКЗ \\
\hline CCNE1 & GSK3B \\
\hline MEP1B & PYY \\
\hline FYN & TNK2 \\
\hline DNTTIP2 & PPARG \\
\hline EXOSC2 & EXOSC7 \\
\hline GNB2L1 & PRKCE \\
\hline SUMO1 & TP73 \\
\hline NTRK2 & SQSTM1 \\
\hline SKIL & TFPI2 \\
\hline ELF2 & RUNX1 \\
\hline FYN & NEDD9 \\
\hline LIG1 & PCNA \\
\hline HSF1 & XRCC6 \\
\hline CBL & RET \\
\hline CTBP1 & IKZF1 \\
\hline SMAD5 & SNRNP70 \\
\hline ZBTB16 & ZNF24 \\
\hline CAV1 & PTGS2 \\
\hline MAP2K6 & MAPK14 \\
\hline ARR3 & SRC \\
\hline
\end{tabular}




\begin{tabular}{|c|c|}
\hline $\mathrm{A} 2 \mathrm{M}$ & HSPA5 \\
\hline NMI & STAT5A \\
\hline JAK1 & TEC \\
\hline GSTO1 & KAT5 \\
\hline CALM1 & PDE1A \\
\hline RYR2 & SRI \\
\hline COPS5 & COPS6 \\
\hline FASTK & MDFI \\
\hline CDC25C & PCNA \\
\hline BMX & CASP3 \\
\hline APOE & LRP2 \\
\hline HDAC1 & PML \\
\hline PYGL & PYGL \\
\hline COPS5 & JUN \\
\hline GTF2F1 & PSMC2 \\
\hline CYP2C19 & POR \\
\hline E2F1 & GSK3B \\
\hline $\mathrm{BCR}$ & PTPN6 \\
\hline ITGA1 & MATN1 \\
\hline SLC9A3 & SLC9A3R2 \\
\hline E2F1 & TOPBP1 \\
\hline TAF1 & TAF6 \\
\hline COPB1 & KDELR1 \\
\hline AKAP9 & PKN1 \\
\hline DLG4 & KCNA5 \\
\hline CDK2 & CDKN3 \\
\hline GTF2B & TBP \\
\hline CXCL10 & DPP4 \\
\hline IFIT1 & IFIT3 \\
\hline MLL & RNF2 \\
\hline RBPMS & TRIP13 \\
\hline HOXB6 & PLSCR1 \\
\hline TGFB3 & TGFBR2 \\
\hline EWSR1 & RPL31 \\
\hline CSNK2A1 & HSP90AA1 \\
\hline MAPK3 & SPIB \\
\hline BRF1 & TP53 \\
\hline KCNB1 & KCNB1 \\
\hline DOK1 & SRC \\
\hline MSX1 & SP1 \\
\hline EIF2AK2 & TP53 \\
\hline DLG4 & MAР3К10 \\
\hline DDR1 & TTR \\
\hline FOX01 & HOXA5 \\
\hline S100A10 & SETDB1 \\
\hline EP300 & ETV1 \\
\hline MAP4K1 & NCK1 \\
\hline
\end{tabular}




\begin{tabular}{|c|c|}
\hline S100A3 & S100A3 \\
\hline MYBL2 & RBL1 \\
\hline CREBBP & KHDRBS1 \\
\hline CSF1R & PIK3R1 \\
\hline NOVA1 & SMARCC2 \\
\hline EPHB2 & SRC \\
\hline BAG1 & NR3C1 \\
\hline EGR1 & PSMA3 \\
\hline MTA1 & RBBP4 \\
\hline HRAS & INSR \\
\hline BIN1 & ITGA1 \\
\hline ATF7 & TAF12 \\
\hline ATP6V1E1 & XRCC6 \\
\hline RXRA & TDG \\
\hline PRKACA & RYR2 \\
\hline BCL6 & JUND \\
\hline FYN & SKAP1 \\
\hline DDO & PEX5 \\
\hline IKBKE & MYH10 \\
\hline MDM4 & YWHAZ \\
\hline CDX2 & EP300 \\
\hline JUN & NCOA1 \\
\hline IGF2 & IGFBP3 \\
\hline GTF2E2 & SND1 \\
\hline JAK3 & STAT5A \\
\hline INSR & IRS1 \\
\hline DNM2 & EPS15 \\
\hline AP2B1 & AP2M1 \\
\hline CAT & CAT \\
\hline PLAT & PLAU \\
\hline EEF1A1 & PAFAH1B3 \\
\hline TRAF6 & ZMYND11 \\
\hline GNAI2 & NUCB1 \\
\hline CBX5 & TAF4 \\
\hline EGFR & MUC1 \\
\hline HNRNPA1 & NFKBIA \\
\hline EPOR & GNAI1 \\
\hline RB1 & TAF1 \\
\hline PSMB2 & PSMB3 \\
\hline EWSR1 & WWP1 \\
\hline EPHB3 & FYN \\
\hline ATXN3 & PSMD7 \\
\hline KLF1 & SMARCB1 \\
\hline PTK2B & RB1CC1 \\
\hline SFPQ & TOP1 \\
\hline GTF2F1 & MYC \\
\hline AHNAK & S100B \\
\hline
\end{tabular}




\begin{tabular}{|c|c|}
\hline MCM6 & MCM7 \\
\hline AKT3 & TCL1A \\
\hline MAP2K1 & PEBP1 \\
\hline PXN & SYK \\
\hline ERBB2 & ERBB3 \\
\hline CTNNB1 & TCF4 \\
\hline ADRM1 & RAD23B \\
\hline ITGA3 & LGALS8 \\
\hline CELSR2 & KLF6 \\
\hline DUSP7 & GHR \\
\hline MYST3 & RUNX1 \\
\hline MEN1 & VIM \\
\hline MEIS1 & PBX1 \\
\hline ETF1 & KEAP1 \\
\hline HSPA2 & MAP3К3 \\
\hline TNFRSF8 & TRAF3 \\
\hline $\mathrm{BCL} 2$ & CASP3 \\
\hline ARHGAP1 & BNIP2 \\
\hline MSH3 & PCNA \\
\hline CASP2 & CASP7 \\
\hline CREBBP & STAT2 \\
\hline IL2RB & JAK1 \\
\hline PRKCA & SLC1A1 \\
\hline FYB & SKAP1 \\
\hline KEAP1 & NFE2L2 \\
\hline FXR2 & TNNT1 \\
\hline HNRNPD & HNRNPD \\
\hline МАРЗКЗ & YWHAH \\
\hline FBLN2 & VCAN \\
\hline LTK & PTPN1 \\
\hline BAT3 & GLRA2 \\
\hline ANP32A & MAP1B \\
\hline MCM5 & RPA2 \\
\hline CALM1 & OPRM1 \\
\hline HNRNPL & РCBP2 \\
\hline RGS2 & SCN5A \\
\hline EED & HDAC2 \\
\hline FLT1 & PLCG1 \\
\hline PEX13 & PEX19 \\
\hline LRP1 & PLAT \\
\hline DNM2 & GRB2 \\
\hline PARP1 & XRCC5 \\
\hline CHML & RAB3A \\
\hline AES & SH3GL3 \\
\hline FKBP5 & PGR \\
\hline CTNND1 & MUC1 \\
\hline E2F1 & SP1 \\
\hline
\end{tabular}




\begin{tabular}{|c|c|}
\hline RUNX1T1 & TCF3 \\
\hline PRSS3 & TFPI \\
\hline ITGB3 & THBS1 \\
\hline IFNG & IFNG \\
\hline MED1 & MED1 \\
\hline TAF5 & TAF7 \\
\hline TXNIP & ZBTB16 \\
\hline CSF1R & GRB2 \\
\hline AKT1 & IRAK1 \\
\hline CCL22 & CCR4 \\
\hline FN1 & SDC2 \\
\hline ACLY & GSK3B \\
\hline BAD & PRKACA \\
\hline DLAT & PDK2 \\
\hline PAK2 & SRC \\
\hline ESRRB & NCOA2 \\
\hline PIN1 & SUPT5H \\
\hline HCLS1 & LYN \\
\hline THBS3 & THBS3 \\
\hline CDC7 & CDK4 \\
\hline RXRG & SRF \\
\hline USP8 & YWHAQ \\
\hline PPP2CA & PRKCD \\
\hline TRIM28 & ZNF74 \\
\hline JAK1 & PTPN11 \\
\hline PPIB & PRL \\
\hline $\mathrm{BCL} 2$ & HRK \\
\hline CRYAB & CRYGC \\
\hline BAT1 & BAT1 \\
\hline CDX1 & CEBPA \\
\hline DNMT1 & $\mathrm{EZH} 2$ \\
\hline GTF2H1 & PSMC2 \\
\hline HDAC1 & NFKBIA \\
\hline KARS & SLC25A6 \\
\hline ANXA7 & SRI \\
\hline RARA & RXRG \\
\hline DUSP4 & MAPK8 \\
\hline SFN & TRIM25 \\
\hline CUL4B & DDB2 \\
\hline NUP98 & RAE1 \\
\hline DUSP3 & MAPK1 \\
\hline CTNNB1 & PTPRK \\
\hline EZR & ICAM3 \\
\hline ADAM15 & $\mathrm{HCK}$ \\
\hline ATXN2 & NCOA4 \\
\hline FASTK & TIA1 \\
\hline BMX & CAV1 \\
\hline
\end{tabular}




\begin{tabular}{|c|c|}
\hline IL2RB & STAT5B \\
\hline PRMT5 & YWHAQ \\
\hline NPY & NPY1R \\
\hline HDAC1 & PTMA \\
\hline CTNND1 & PSEN1 \\
\hline АСТВ & СCT5 \\
\hline ARF6 & CHRM3 \\
\hline HDAC2 & RELA \\
\hline BLMH & UBE2I \\
\hline $\mathrm{CP}$ & LTF \\
\hline LASP1 & TRIP13 \\
\hline CCDC85B & SF3A3 \\
\hline PAX5 & RUNX1 \\
\hline ID2 & MYF6 \\
\hline АСТВ & PFN1 \\
\hline APPBP2 & CNTFR \\
\hline ACP1 & KDR \\
\hline EIF4E & EIF4G1 \\
\hline MCM7 & MCM7 \\
\hline SMAD3 & SNRNP70 \\
\hline$A R$ & EP300 \\
\hline ATXN1 & PUM1 \\
\hline ATXN1 & PTGDS \\
\hline PPP2CA & PPP2R1A \\
\hline LMO4 & RBBP8 \\
\hline TAF1 & TAF1 \\
\hline ID2 & MYOD1 \\
\hline ANP32A & AXIN1 \\
\hline GNA15 & RGS2 \\
\hline FGF1 & FGF1 \\
\hline PLP2 & PLP2 \\
\hline CYBA & NCF4 \\
\hline NAPA & STX5 \\
\hline LMNB1 & TMPO \\
\hline CCT7 & KAT5 \\
\hline CD5 & PTPN6 \\
\hline ETV1 & MAPKAPK2 \\
\hline EP300 & GTF2B \\
\hline MDFI & SPG7 \\
\hline PIP4K2B & TNFRSF1A \\
\hline ACTG1 & SH3GL2 \\
\hline RUNX1 & YAP1 \\
\hline HSPG2 & NID2 \\
\hline APBA1 & KCNJ12 \\
\hline CREB5 & CREB5 \\
\hline CDK5 & STXBP1 \\
\hline GRK5 & $\mathrm{RHO}$ \\
\hline
\end{tabular}




\begin{tabular}{|c|c|}
\hline ATF4 & JUN \\
\hline KIT & LYN \\
\hline HSF1 & HSF2 \\
\hline MCM2 & MCM5 \\
\hline CKM & FHL2 \\
\hline CASP8 & TFCP2 \\
\hline BMP2 & BMPR1B \\
\hline EP300 & RUNX1 \\
\hline KIF2C & KIF2C \\
\hline PPP2CA & PPP2R2A \\
\hline BMI1 & PHC2 \\
\hline CRP & FN1 \\
\hline AVPR2 & GRK5 \\
\hline LRP1 & MDK \\
\hline AP2M1 & CD22 \\
\hline PTPRH & PXN \\
\hline HNRNPK & MATR3 \\
\hline ADD1 & HSPH1 \\
\hline DCC & NCK1 \\
\hline CFL1 & YWHAZ \\
\hline EP300 & MEF2A \\
\hline BIN1 & ITGA3 \\
\hline DLG4 & GRIN2B \\
\hline HDAC2 & RB1 \\
\hline EIF4E & MKNK1 \\
\hline CREBBP & NCOA1 \\
\hline SH3BP2 & ZAP70 \\
\hline GNB2L1 & JAK1 \\
\hline CAV1 & PTPRF \\
\hline ACTG1 & BCAP31 \\
\hline DSG2 & PKP2 \\
\hline RASA1 & SLC9A2 \\
\hline FANCC & GSTP1 \\
\hline PTPN3 & YWHAB \\
\hline CBL & PTK2B \\
\hline CSNK2A2 & HSP90AA1 \\
\hline RPLP1 & RPLP2 \\
\hline CRYAA & CRYAA \\
\hline PTPN12 & SHC1 \\
\hline PPFIA1 & PTPRS \\
\hline NGFR & TRADD \\
\hline $\mathrm{BBC} 3$ & MCL1 \\
\hline ADD1 & ID3 \\
\hline BRCA2 & RPA1 \\
\hline EFNA3 & EPHA7 \\
\hline NFIX & SKI \\
\hline PAFAH1B3 & UNC119 \\
\hline
\end{tabular}




\begin{tabular}{|c|c|}
\hline PLG & SERPINF2 \\
\hline TAX1BP1 & TRAF6 \\
\hline FASLG & SUMO1 \\
\hline TNFRSF1B & TRAF1 \\
\hline FKBP5 & HSP90AA1 \\
\hline GNB2L1 & PLCG1 \\
\hline DAG1 & SHC1 \\
\hline BCL2L1 & BECN1 \\
\hline ACVR1 & BMP7 \\
\hline ESR1 & PRMT2 \\
\hline PHLDA1 & PLSCR1 \\
\hline FYN & SRC \\
\hline ASL & QARS \\
\hline FEZ1 & NBR1 \\
\hline SKIL & TRAK1 \\
\hline COPS6 & EIF3E \\
\hline PIAS1 & TP53 \\
\hline ATF3 & JUND \\
\hline CREBBP & MSX1 \\
\hline КАТ2B & RBPJ \\
\hline АРPBP2 & RRS1 \\
\hline MAPK3 & $\mathrm{TH}$ \\
\hline PLCG1 & PRKD1 \\
\hline CANX & $\mathrm{EBI3}$ \\
\hline HSP90AA1 & MYOD1 \\
\hline FUBP1 & TLE1 \\
\hline ATXN1 & HSPA1A \\
\hline PLSCR1 & SHC1 \\
\hline CCL22 & DPP4 \\
\hline MED24 & SMAD2 \\
\hline TPD52L1 & YWHAZ \\
\hline KRT18 & KRT5 \\
\hline CUL7 & SKP1 \\
\hline CASP10 & CASP8 \\
\hline CHRNA1 & CHRNE \\
\hline HDLBP & SMAD4 \\
\hline PIK3C3 & PIK3R4 \\
\hline EIF2S2 & TLE1 \\
\hline KPNB1 & NUP98 \\
\hline AR & BRCA1 \\
\hline PKD1 & SIAH1 \\
\hline FHL3 & LASP1 \\
\hline AP2M1 & FURIN \\
\hline CCL11 & CCR3 \\
\hline СТВP1 & EP300 \\
\hline EWSR1 & HERPUD1 \\
\hline DOK1 & YES1 \\
\hline
\end{tabular}




\begin{tabular}{|c|c|}
\hline DLG4 & ERBB4 \\
\hline CDC25B & MELK \\
\hline SCNN1G & STX1A \\
\hline HRAS & PIK3CA \\
\hline E2F5 & RBL1 \\
\hline ST6GAL1 & ST6GAL1 \\
\hline G3BP1 & G3BP1 \\
\hline EP300 & SMAD3 \\
\hline BTK & $\mathrm{CBL}$ \\
\hline G6PD & G6PD \\
\hline INSR & PTPN11 \\
\hline EIF2S2 & PRKDC \\
\hline PSMB1 & PSMB7 \\
\hline ITPR3 & TRPC3 \\
\hline CXCL1 & DARC \\
\hline FHL2 & ZNF638 \\
\hline FOSL1 & JUN \\
\hline GTF2E1 & GTF2H1 \\
\hline FUBP1 & SMN1 \\
\hline SMARCC1 & WWOX \\
\hline POLR2E & POLR2K \\
\hline DDB1 & VAMP3 \\
\hline EEF1A1 & MLLT3 \\
\hline BRCA2 & FLNA \\
\hline NCOR2 & RUNX1T1 \\
\hline MEP1A & NTS \\
\hline MAPT & OGT \\
\hline PRKCSH & PTPRC \\
\hline GNB3 & GNG4 \\
\hline BAT3 & STX5 \\
\hline NFKBIE & REL \\
\hline INSM1 & MPP3 \\
\hline KAT5 & SNRPD2 \\
\hline CPSF6 & PLSCR1 \\
\hline GBP2 & PPP1R8 \\
\hline CBFB & RUNX1 \\
\hline SYN1 & VIM \\
\hline PRKCG & TIAM1 \\
\hline CD74 & CD74 \\
\hline MARK3 & YWHAZ \\
\hline EFNB2 & ЕРHB2 \\
\hline CANX & EDEM1 \\
\hline NCK1 & PAK1 \\
\hline AES & AR \\
\hline CRMP1 & SAT1 \\
\hline BCAP31 & MYH10 \\
\hline CCR5 & CXCR4 \\
\hline
\end{tabular}




\begin{tabular}{|c|c|}
\hline ERCC3 & XPC \\
\hline AP2M1 & AQP4 \\
\hline CAMLG & EGFR \\
\hline CALCOCO2 & DAZAP2 \\
\hline ANXA6 & S100B \\
\hline GP1BA & GP5 \\
\hline KCNJ3 & KCNJ5 \\
\hline AMPH & SH3GL2 \\
\hline BRCA1 & CCNA1 \\
\hline CBX5 & CHAF1A \\
\hline PF4 & PROC \\
\hline PPP1CC & PPP1R8 \\
\hline TAF1 & TAF12 \\
\hline S100P & S100P \\
\hline CASP3 & CFLAR \\
\hline CIITA & RFX5 \\
\hline MAPK7 & MEF2C \\
\hline BIN1 & ITGA6 \\
\hline $\mathrm{MCC}$ & NFKBIB \\
\hline HMGB1 & RB1 \\
\hline FLII & MYD88 \\
\hline CITED1 & SMAD4 \\
\hline EGFR & PTPN1 \\
\hline GGA3 & TSG101 \\
\hline POLR2B & POLR2C \\
\hline SNAPC2 & TBP \\
\hline VIM & VIM \\
\hline C1QBP & PRKCA \\
\hline FHL2 & MCM7 \\
\hline FN1 & MEP1B \\
\hline SH3GL2 & TCEA2 \\
\hline FADD & TRADD \\
\hline PTPN6 & STAT5B \\
\hline PLCG1 & SELE \\
\hline CRMP1 & TK1 \\
\hline SUMO2 & SUMO2 \\
\hline CCND3 & CDK6 \\
\hline JUND & MAPK8 \\
\hline SMARCA4 & SMARCB1 \\
\hline BAT1 & DDX39 \\
\hline GNAI2 & MDFI \\
\hline MAP2K3 & PLCB2 \\
\hline CDK9 & TP53 \\
\hline CREBBP & SND1 \\
\hline HOXB1 & PBX1 \\
\hline JUN & TAF1 \\
\hline FGFR3 & SMG7 \\
\hline
\end{tabular}




\begin{tabular}{|c|c|}
\hline GTF2E1 & GTF2H4 \\
\hline BRCA1 & STAT1 \\
\hline FUS & PRMT1 \\
\hline CREBBP & VDR \\
\hline FGA & NID1 \\
\hline AMPH & SYN1 \\
\hline CD36 & ITGA6 \\
\hline MAP2K3 & MAPK8IP2 \\
\hline CD79A & HCLS1 \\
\hline MED24 & PPARA \\
\hline JUN & MYOD1 \\
\hline LMNA & SMAD3 \\
\hline CD44 & MMP7 \\
\hline AXIN1 & GSK3B \\
\hline ELL & TP53 \\
\hline CUL1 & SKP1 \\
\hline MCM2 & MCM3 \\
\hline CRYAB & CRYBB2 \\
\hline RB1 & UBTF \\
\hline MEN1 & RELA \\
\hline YAF2 & YY1 \\
\hline LYN & UNC119 \\
\hline ATN1 & DMPK \\
\hline PAFAH1B1 & PAFAH1B2 \\
\hline KLF1 & SMARCC1 \\
\hline EPHB1 & GRB2 \\
\hline CAPN1 & PSEN2 \\
\hline DDX17 & NCOA1 \\
\hline CTLA4 & CTLA4 \\
\hline VEGFA & VEGFA \\
\hline ATXN1 & OAZ1 \\
\hline VAV1 & XRCC5 \\
\hline RELN & RELN \\
\hline BRCA1 & МАРЗКЗ \\
\hline HMGA1 & NFYA \\
\hline MAPK10 & TP53 \\
\hline CUL1 & RAC2 \\
\hline MCM5 & MCM7 \\
\hline INSR & PTPN6 \\
\hline MECP2 & SMARCB1 \\
\hline NEDD8 & UCHL3 \\
\hline PRMT1 & SUPT5H \\
\hline CD247 & DOCK2 \\
\hline PPARA & RELA \\
\hline NEB & TTN \\
\hline CTNNB1 & MAGI1 \\
\hline PPIF & SLC25A4 \\
\hline
\end{tabular}




\begin{tabular}{|c|c|}
\hline HOXA9 & MEIS1 \\
\hline FGF9 & FGFR3 \\
\hline MAPK3 & TP53 \\
\hline NROB2 & $\mathrm{NR} 1 \mathrm{H} 2$ \\
\hline ESR1 & MDM2 \\
\hline GRB2 & KDR \\
\hline KHDRBS1 & PIK3R1 \\
\hline CD72 & SEMA4D \\
\hline HTT & XRCC6 \\
\hline DAB2 & TGFBR2 \\
\hline PBX2 & RPL4 \\
\hline ACTA1 & CCT4 \\
\hline RARA & TRIP4 \\
\hline GHR & PTPN1 \\
\hline CDC42EP1 & PLSCR1 \\
\hline APOB & BGN \\
\hline PDLIM4 & RBPMS \\
\hline ACTN1 & ACTN1 \\
\hline GPS2 & SETDB1 \\
\hline CRK & PTPN1 \\
\hline NAPA & SNAP23 \\
\hline CDC7 & MCM7 \\
\hline PSMC5 & THRB \\
\hline NFKBIA & TP53 \\
\hline BCL2A1 & PMAIP1 \\
\hline CALM1 & RALB \\
\hline CUL2 & VHL \\
\hline MAPK8 & PRKD1 \\
\hline SERPINB9 & TLE1 \\
\hline ITGB1BP1 & LRP2 \\
\hline AR & IFI16 \\
\hline FUS & RXRA \\
\hline GUCY1A2 & GUCY1B3 \\
\hline KAT2B & TTF1 \\
\hline CDK2 & PCNA \\
\hline BRCA1 & CDK2 \\
\hline PRKCl & SMG1 \\
\hline CD5 & ZAP70 \\
\hline TAF11 & TAF6 \\
\hline MTFR1 & PIN1 \\
\hline MAP3K5 & TRAF5 \\
\hline PPP4C & REL \\
\hline PAFAH1B3 & SETDB1 \\
\hline AXIN1 & CTNNB1 \\
\hline DAZAP2 & WWP1 \\
\hline CTSG & SDC1 \\
\hline RIT2 & RLF \\
\hline
\end{tabular}




\begin{tabular}{|c|c|}
\hline АСТВ & RAC1 \\
\hline APLP1 & SAT1 \\
\hline ACTB & CFL1 \\
\hline ANK1 & L1CAM \\
\hline FHL2 & ITGB6 \\
\hline HNRNPU & NDN \\
\hline CCDC85B & HNRNPC \\
\hline CALCOCO2 & PSMA1 \\
\hline CDKN1A & GNB2L1 \\
\hline $\mathrm{BCL3}$ & NFKB1 \\
\hline LRP1 & SRC \\
\hline NFYB & TBP \\
\hline HNRNPC & PIN1 \\
\hline F8 & LRP1 \\
\hline GRB2 & USP6NL \\
\hline ERCC1 & XPA \\
\hline CD2 & CD53 \\
\hline NTRK3 & SQSTM1 \\
\hline CNTFR & LIFR \\
\hline KRT1 & PRKCE \\
\hline FCAR & FCGR1A \\
\hline HDAC1 & MDM2 \\
\hline ALOX5 & GRB2 \\
\hline CDK9 & CUL1 \\
\hline BNIP3 & NCBP1 \\
\hline GGA3 & RPS27A \\
\hline CA9 & $\mathrm{CDH} 1$ \\
\hline CD24 & SELP \\
\hline BRCA1 & HDAC1 \\
\hline NCOR2 & PPARD \\
\hline TP73 & TP73 \\
\hline MMP2 & THBS2 \\
\hline ACY1 & TTC1 \\
\hline CCNC & CDK8 \\
\hline DGUOK & DGUOK \\
\hline DAB2 & PIN1 \\
\hline COPS8 & COPS8 \\
\hline ARHGDIA & SH3GL3 \\
\hline DNM1 & ITSN1 \\
\hline BMPR1B & BMPR1B \\
\hline PTPN11 & STAT3 \\
\hline$A R$ & GSN \\
\hline CSF2RB & IL5RA \\
\hline GNA13 & RDX \\
\hline CSK & FYN \\
\hline GRB10 & INSR \\
\hline BET1 & STX5 \\
\hline
\end{tabular}




\begin{tabular}{|c|c|}
\hline AR & CDK7 \\
\hline CALCOCO2 & LGALS8 \\
\hline MAPK1 & SREBF2 \\
\hline MAPKAPK2 & SHC1 \\
\hline POLR2G & TAF15 \\
\hline $\mathrm{CDH} 3$ & CTNND1 \\
\hline GFAP & PSEN1 \\
\hline HRAS & LGALS1 \\
\hline TP53 & TP53BP1 \\
\hline DVL1 & EPS8 \\
\hline$A R$ & CALM1 \\
\hline PLAU & PLAUR \\
\hline EP300 & YY1 \\
\hline EEF1G & SNRPD2 \\
\hline KLK2 & SERPINB6 \\
\hline TRIM23 & TRIM31 \\
\hline XBP1 & XBP1 \\
\hline GSK3B & PSEN1 \\
\hline APOA4 & GPLD1 \\
\hline CRHR1 & GNAO1 \\
\hline JAK1 & STAT3 \\
\hline$A R$ & NR2C1 \\
\hline GRIN2B & IL16 \\
\hline EEF1G & TUBB3 \\
\hline PSME1 & VIM \\
\hline CAV1 & EGFR \\
\hline ERBB2 & PIK3R1 \\
\hline AKT1 & IRS1 \\
\hline GAD2 & PRKCE \\
\hline IGF1R & IRS1 \\
\hline ATF4 & CCDC106 \\
\hline EMD & SH3GL2 \\
\hline CCNO & RPA2 \\
\hline BAT2 & EIF3E \\
\hline AR & GTF2H1 \\
\hline RELA & TBP \\
\hline $\mathrm{CDH} 1$ & HDAC1 \\
\hline IL7R & PTK2B \\
\hline TFRC & TFRC \\
\hline KPNA4 & TGM2 \\
\hline NPM1 & TFAP2A \\
\hline PTK2B & SRC \\
\hline CDK5R1 & SET \\
\hline ATXN1 & CFL1 \\
\hline CREBBP & PML \\
\hline KPNB1 & NUP62 \\
\hline AKT1 & MDM2 \\
\hline
\end{tabular}




\begin{tabular}{|c|c|}
\hline QARS & RARS \\
\hline NF2 & NF2 \\
\hline DYNLL1 & DYNLL1 \\
\hline DAP & SETDB1 \\
\hline LYN & MAPK3 \\
\hline ERCC5 & NTHL1 \\
\hline PRKCI & RAB4A \\
\hline LCP2 & WAS \\
\hline $\mathrm{OGDH}$ & PLSCR1 \\
\hline SRC & TRAF1 \\
\hline PGM1 & S100B \\
\hline MAPK14 & MEF2A \\
\hline ID2 & UNC119 \\
\hline DSC2 & PKP2 \\
\hline NCF4 & TXN \\
\hline BRCA1 & LMO4 \\
\hline CTNNB1 & SMARCA4 \\
\hline FGFR3 & POLA2 \\
\hline ERCC3 & GTF2E2 \\
\hline TDG & THRA \\
\hline FCAR & LYN \\
\hline SFPQ & SMAD5 \\
\hline ITGB3BP & NFKB1 \\
\hline SNRPD1 & SNRPD2 \\
\hline ATP2A1 & SLN \\
\hline CCR3 & FGR \\
\hline EIF4G1 & MKNK1 \\
\hline F12 & GP1BA \\
\hline PCBP2 & РCBP2 \\
\hline CD63 & TIMP1 \\
\hline ANXA5 & KDR \\
\hline MEOX2 & VIP \\
\hline CD36 & CD9 \\
\hline APOA2 & APOD \\
\hline SKP2 & WEE1 \\
\hline BAT2 & GRB2 \\
\hline GRB2 & IL2RB \\
\hline MCM6 & SSRP1 \\
\hline CNTF & LIFR \\
\hline MPRIP & MPRIP \\
\hline ALDOB & ATP6V1E1 \\
\hline ALB & FCGRT \\
\hline MCM5 & ORC2L \\
\hline NEK9 & SSRP1 \\
\hline
\end{tabular}

